\title{
Stereoselective Construction of $\gamma$-Lactams via Copper-Catalyzed Borylacylation
}

\author{
Alexa Torelli, Andrew Whyte, Iuliia Polishchuk, Jonathan Bajohr \\ and Mark Lautens* \\ mark.lautens@utoronto.ca \\ Department of Chemistry, University of Toronto, 80 St. George Street, \\ Toronto, Ontario, M5S 3H6, Canada
}




\section{Supporting Information}

\section{Contents}

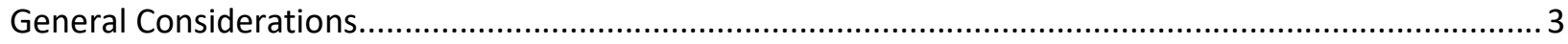

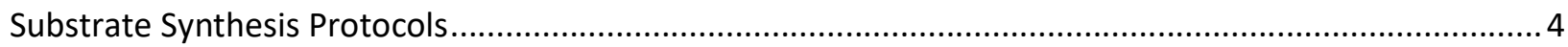

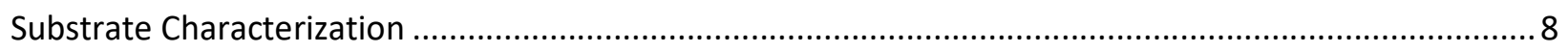

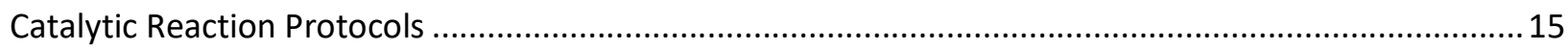

Preliminary Chiral Ligand Screen for Enantioselective Variant......................................................... 16

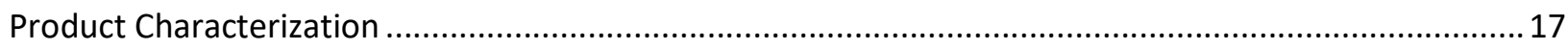

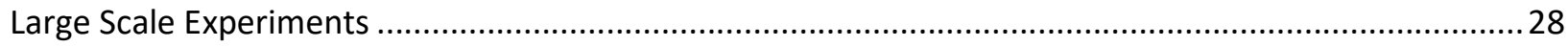

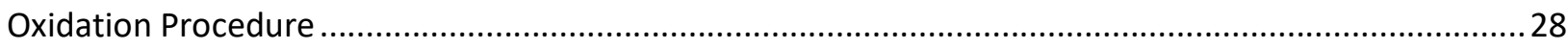

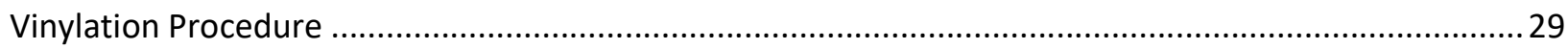

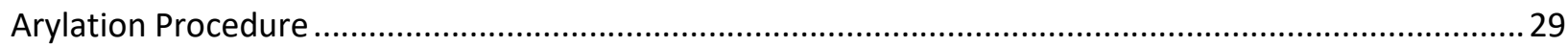

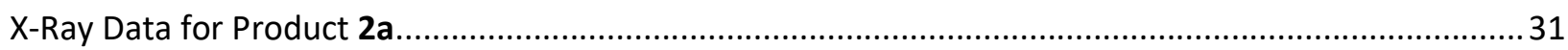

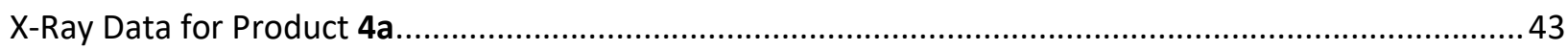

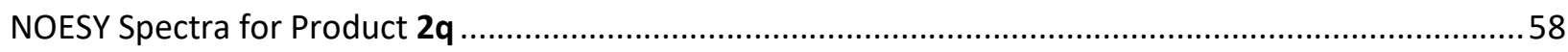

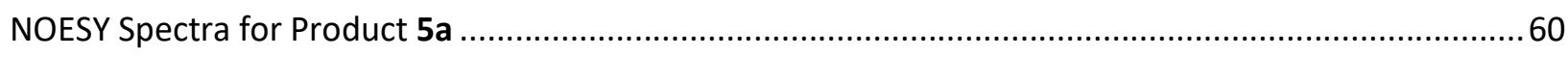

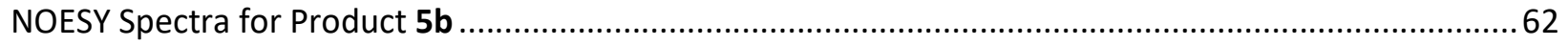

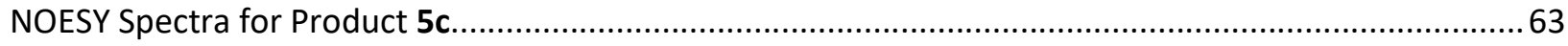

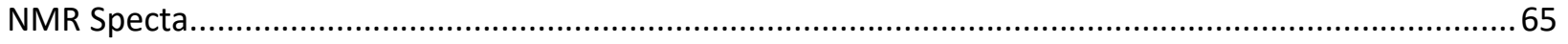




\section{General Considerations}

Reactions were performed under argon in a dry environment unless otherwise stated. Reaction progress was monitored by thin layer chromatography (TLC) and visualized under UV light. THF was distilled over sodium/benzophenone. MTBE was dried over $4 \AA$ molecular sieves. HPLC grade hexanes and isopropanol were purchased from Fisher. Sodium tert-butoxide (97\%) was purchased from Sigma Aldrich and was stored in a dessicator. Copper tetrakisacetonitrile hexafluorophosphate and $(S, S)$-BDPP was purchased from Strem and stored in a dessicator. dppe was purchased form Combi-Blocks and stored in a dessicator. 1,3,5-Trimethoxybenzene was purchased from Sigma Aldrich and used as received. All other starting materials and reagents were purchased from Sigma, Alfa Aesar, Fisher, or Combi-Blocks and were used as received. Catalytic reactions were performed in 2 dram vials equipped with a teflon septa (ThermoScientific National B7995-15) and a stir bar (Fisher cat no. 14-513-57, 12 x 4.5 mm). Flash column chromatography was performed with Silicycle 46-60 $\mu \mathrm{m}$ silica gel.

${ }^{1} \mathrm{H}$ and ${ }^{13} \mathrm{C}$ NMR were obtained at $296 \mathrm{~K}$ on Agilent DD2 500 equipped with a $5 \mathrm{~mm}$ Xses Cold Probe, and ${ }^{19} \mathrm{~F}$ NMR were obtained on a Bruker Avance III 400. Measurements were referenced to the solvent. NMR data is referenced as chemical shift $(\delta \mathrm{ppm})$, multiplicity $(\mathrm{s}=$ singlet, $\mathrm{d}=$ doublet, $\mathrm{t}=$ triplet, $\mathrm{q}=$ quartet, $\mathrm{m}=$ multiplet, $\mathrm{b}=$ broad), coupling constant $(\mathrm{Hz})$, integration. Carbons directly bounded to boron were not observed due to quadrupolar relaxation. NMR yields were obtained by ${ }^{1} \mathrm{H}$ NMR analysis using a 2 second relaxation delay and 1,3,5-trimethoxybenzene as an internal standard. HRMS were obtained on a JEOL AccuTOF-DART performed at the Advanced Instrumentation for Molecular Structure (AIMS) at the University of Toronto. IR spectra were acquired on a Perkin-Elmer Spectrum 100 instrument with a single-bounce diamond/ZeSe ATR accessory. Data is presented in wavenumbers $\left(\mathrm{cm}^{-1}\right)$. Chiral HPLC was performed on Agilent 1100 or 1200 series operated by ChemStation LC 3D software. 


\section{Substrate Synthesis Protocols}

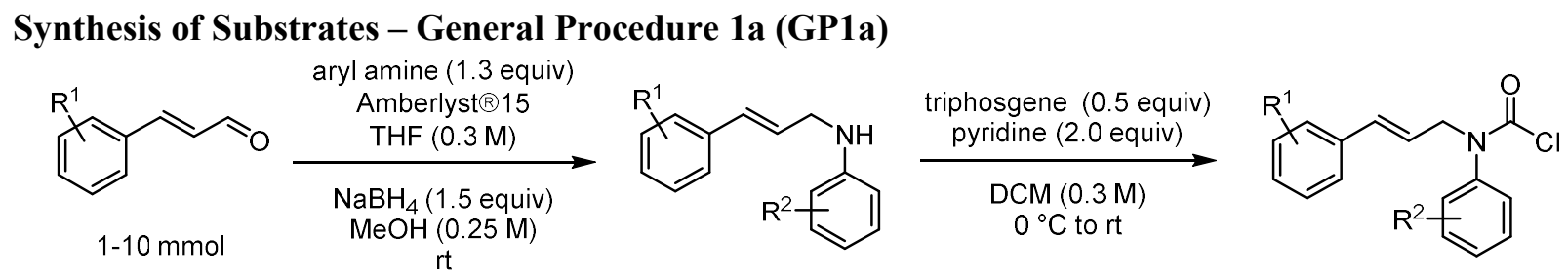

Scheme S1.

GP1a for substrates 1a-o (Scheme S1): Reactions were typically performed on 1-10 mmol scale. Cinnamaldehydes were dissolved in THF $(0.3 \mathrm{M})$ and the corresponding aryl amine (1.3 equiv) was added followed by Amberlyst ${ }^{\circledR} 15$. The reaction was stirred at room temperature until full conversion of the aldehyde to the imine was observed by TLC (typically $30 \mathrm{~min}$ ). Sodium borohydride (1.5 equiv) was added followed by slow addition of $\mathrm{MeOH}(0.25 \mathrm{M})$. The reaction was stirred at room temperature until completion was observed by TLC (typically 1 to $2 \mathrm{~h}$ ). The reaction was concentrated and filtered over a pad of silica using EtOAc. The corresponding amine products were concentrated in vacuo and subsequently dissolved in DCM $(0.3 \mathrm{M})$ and cooled to $0{ }^{\circ} \mathrm{C}$. Pyridine $\left(2.0\right.$ equiv) was added at $0{ }^{\circ} \mathrm{C}$, followed by slow addition of triphosgene ( 0.5 equiv). The reaction was warmed to room temperature and stirred until completion was observed by TLC (typically 1 to $3 \mathrm{~h}$ ). The reaction was quenched with $1 \mathrm{M}$ $\mathrm{HCl}$ and diluted with DCM. The aqueous layer was extracted three times with DCM. The organic layer was dried over magnesium sulfate. The carbamoyl chlorides were purified by flash chromatography using $40 \% \rightarrow 60 \% \mathrm{DCM} /$ Pentanes.

\section{Synthesis of Substrates - General Procedure 1b (GP1b)}

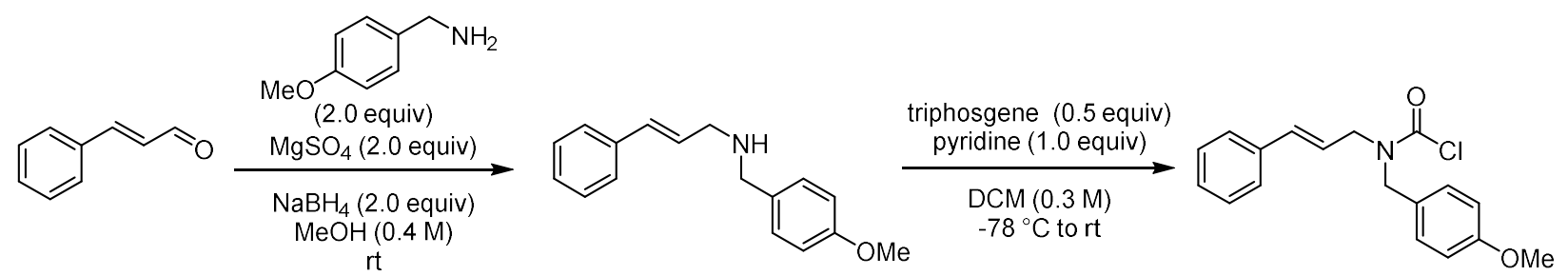

Scheme S2.

GP1b for substrate 10 (Scheme S2): Reaction was performed on $5 \mathrm{mmol}$ scale. Cinnamaldehyde was dissolved in $\mathrm{MeOH}\left(0.4 \mathrm{M}\right.$ ) and 4-methoxybenzylamine (2.0 equiv) was added followed by $\mathrm{MgSO}_{4}$. The reaction was stirred at room temperature until full conversion of the aldehyde to the imine was observed by TLC then sodium borohydride ( 2.0 equiv) was added. The reaction was stirred at room temperature until completion was observed by TLC. The reaction was quenched with $\mathrm{NH}_{4} \mathrm{Cl}$, diluted with EtOAc and extracted thrice. The corresponding amine was concentrated in vacuo and subsequently dissolved in DCM $(0.3 \mathrm{M})$ and cooled to $-78^{\circ} \mathrm{C}$. Pyridine $(1.0$ equiv) was added, followed by slow addition of triphosgene ( 0.5 equiv). The reaction was warmed to room temperature and stirred until completion was observed by TLC. The reaction was quenched with $1 \mathrm{M} \mathrm{HCl}$ and diluted with DCM. The aqueous layer was extracted three times with DCM. The organic layer was dried over magnesium sulfate. The carbamoyl chloride was purified by flash chromatography using $2 \% \rightarrow 10 \%$ EtOAc/Pentanes.

\section{Synthesis of Substrates - General Procedure 1c (GP1c)}




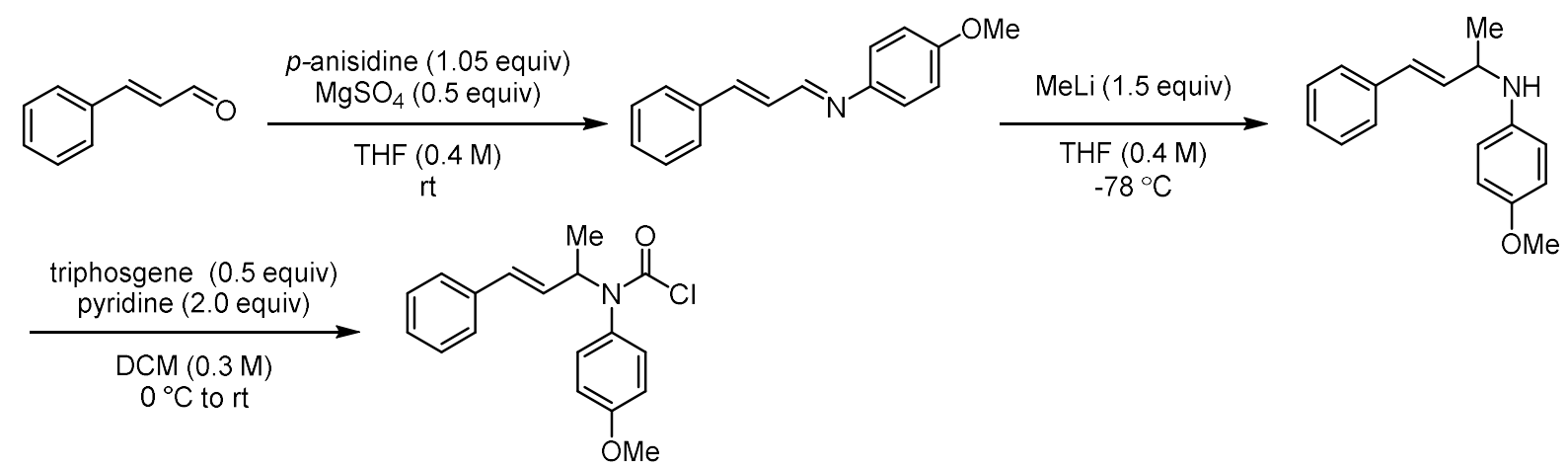

\section{Scheme S3.}

GP1c for substrate 1q (Scheme S3): Reaction was performed on a $36 \mathrm{mmol} \mathrm{scale.} \mathrm{Cinnamaldehyde} \mathrm{was}$ dissolved in THF ( $0.4 \mathrm{M})$ and magnesium sulfate was added ( 0.5 equiv) along with $p$-anisidine (1.05 equiv). The reaction was stirred at room temperature until full conversion to the imine was observed by TLC. The reaction was filtered over a pad of silica and with EtOAc and concentrated in vacuo. The imine was redissolved in THF $(0.4 \mathrm{M})$ and cooled to $-78^{\circ} \mathrm{C}$. At $-78^{\circ} \mathrm{C}$, $\mathrm{MeLi}(1.5$ equiv, $1.6 \mathrm{M}$ in ether) was added dropwise. The reaction was stirred for 30 minutes at $-78^{\circ} \mathrm{C}$ and then quenched with equal volume of saturated $\mathrm{NH}_{4} \mathrm{Cl}$. The organic layer was washed, concentrated, and passed though a short plug of silica with EtOAc/Pentane eluent. Without further purification, the amine was subject to acylation according to GP1a. 


\section{Synthesis of Substrates - General Procedure 2a (GP2a)}
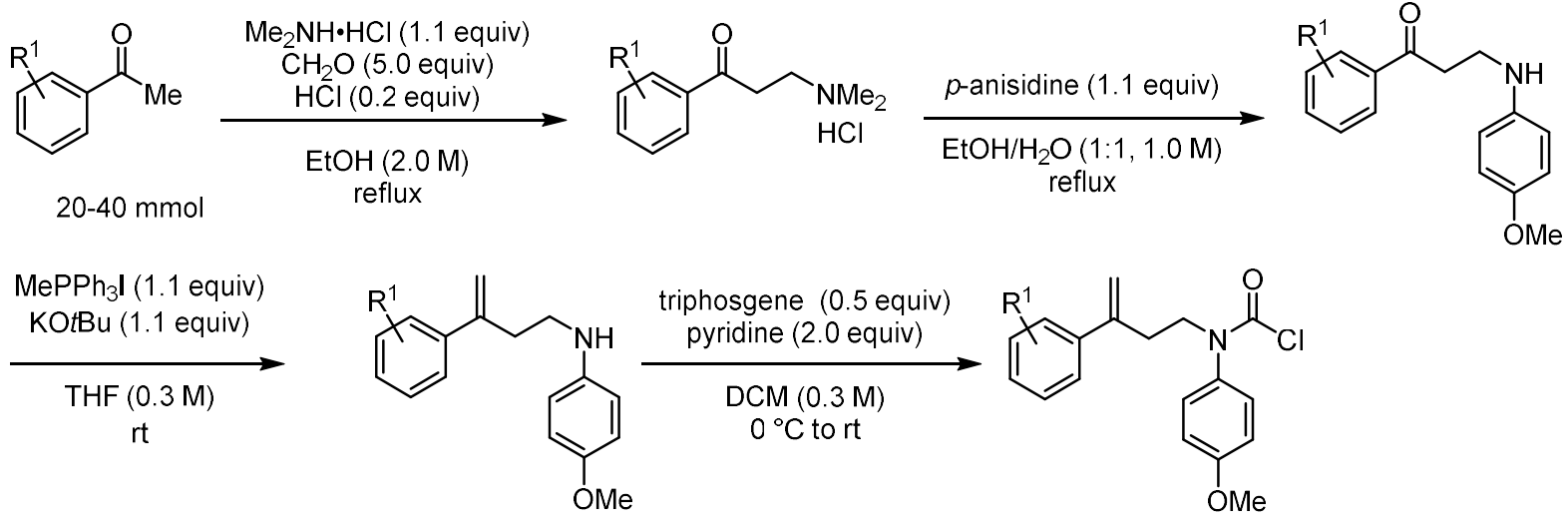

Scheme S4.

GP2a for substrates 3a-f (Scheme S4): A solution of methyl ketone, dimethylamine hydrochloride (1.1 equiv), and paraformaldehyde (5.0 equiv) in $\mathrm{EtOH}(2.0 \mathrm{M})$ was stirred and conc. $\mathrm{HCl}(0.2$ equiv) was added. The reaction was stirred at reflux in an oil bath for approximately 48 hours, then cooled to room temperature and concentrated in vacuo. EtOAc was added and stirred at room temperature for about 15 minutes to allow for the salt to precipitate out. The suspension was filtered and washed with EtOAc twice and dried under nitrogen flow. The dimethylamino ketone hydrochloride salt was subsequently dissolved in $1: 1$ mixture of $\mathrm{H}_{2} \mathrm{O}$ and $\mathrm{EtOH}(0.1 \mathrm{M})$ and $p$-anisidine (1.1 equiv) was added and stirred at reflux in an oil bath for approximately 72 hours. The reaction was cooled to room temperature and the precipitate was filtered and washed twice with $\mathrm{H}_{2} \mathrm{O}$ and twice with EtOH. The aminoketone was dried under a nitrogen sweep. The Wittig olefination was commenced by dissolving $\mathrm{MePPh}_{3} \mathrm{I}(1.1$ equiv) in $\operatorname{THF}(0.3 \mathrm{M})$ and adding $\mathrm{KO} t \mathrm{Bu}$ (1.1 equiv) at room temperature. After 15 minutes, the aminoketone was added slowly. The reaction was stirred at room temperature until completion was observed by TLC (approximately $3 \mathrm{~h}$ ). The reaction was filtered directly over a pad of silica with $30 \%$ EtOAc/pentane mixture. The 1,1disubstituted olefins were subsequently dissolved in DCM $(0.3 \mathrm{M})$ and cooled to $0{ }^{\circ} \mathrm{C}$. Pyridine $(2.0$ equiv) was added at $0{ }^{\circ} \mathrm{C}$, followed by slow addition of triphosgene ( 0.5 equiv). The reaction was warmed to room temperature and stirred until completion was observed by TLC (typically 1 to $3 \mathrm{~h}$ ). The reaction was quenched with $1 \mathrm{M} \mathrm{HCl}$ and diluted with DCM. The aqueous layer was extracted three times with DCM. The organic layer was dried over magnesium sulfate. The carbamoyl chlorides were purified by flash chromatography using $30 \% \rightarrow 60 \%$ DCM/Pentanes.

\section{Synthesis of Substrates - General Procedure 2b}

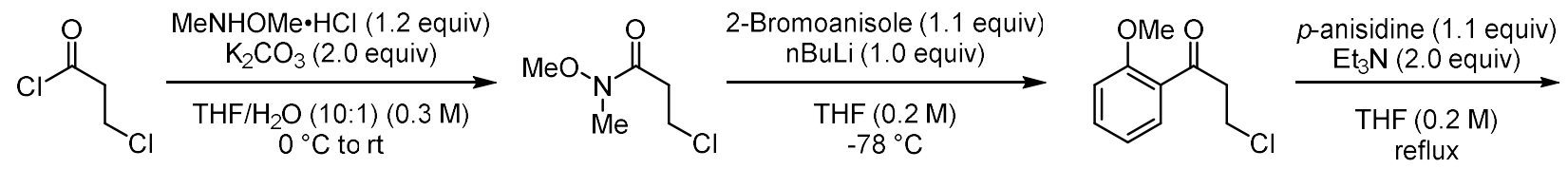<smiles>COc1ccc(NCCC(=O)c2ccccc2OC)cc1</smiles>

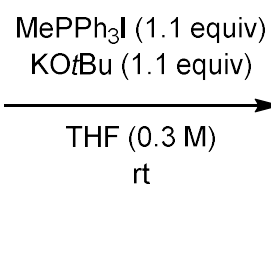<smiles>C=C(CCNc1ccc(OC)cc1)c1ccccc1OC</smiles>

triphosgene ( 0.5 equiv) pyridine (2.0 equiv)

$\operatorname{DCM}(0.3 \mathrm{M})$ $0{ }^{\circ} \mathrm{C}$ to rt<smiles>C=C(CCN(C(=O)Cl)c1ccc(OC)cc1)c1ccccc1OC</smiles>

Scheme S5. 
GP2b for substrate $\mathbf{3 b}$ (Scheme S5): N,O-Dimethylhydroxylamine hydrochloride (1.2 equiv) was dissolved in THF and water $(10: 1)(0.3 \mathrm{M}) . \mathrm{K}_{2} \mathrm{CO}_{3}$ (2.0 equiv) was added and the mixture cooled to $0{ }^{\circ} \mathrm{C}$. The acid chloride was added at $0{ }^{\circ} \mathrm{C}$ then the mixture warmed up to room temperature and stirred overnight. The reaction was quenched with $\mathrm{H}_{2} \mathrm{O}$ and diluted with EtOAc. The aqueous layer was extracted with EtOAc twice, and the organic layer was washed once with $\mathrm{H}_{2} \mathrm{O}$, then concentrated and dried under vacuum. The solid was dissolved in a in minimal amount of THF and added dropwise to a solution of 2-Bromoanisole (1.5 equiv) dissolved in THF $(0.2 \mathrm{M})$ cooled to $-78^{\circ} \mathrm{C}$. ${ }^{\mathrm{n}} \mathrm{BuLi}$ (1.0 equiv, 2.5 $\mathrm{M}$ in hexane) was added dropwise and the reaction was stirred at $-78{ }^{\circ} \mathrm{C}$ for 45 minutes. The mixture was stirred at $-78{ }^{\circ} \mathrm{C}$ until completion was observed by TLC. The mixture was quenched with $\mathrm{H}_{2} \mathrm{O}$ and diluted with EtOAc. The aqueous layer was extracted with EtOAc twice, and the organic layer was washed once with $\mathrm{H}_{2} \mathrm{O}$. The ketone was dissolved in THF $(0.3 \mathrm{M})$ along with $p$-anisidine (1.1 equiv) and $\mathrm{NEt}_{3}(2.0$ equiv). The reaction was stirred at reflux in an oil bath until completion was observed by TLC (approximately 8 hours). The reaction was cooled and directly filtered over a pad of silica using a mixture of EtOAc/pentane. Subsequent olefination and acylation were conducted according to GP2a. The carbamoyl chloride was purified by flash chromatography using $30 \% \rightarrow 80 \% \mathrm{DCM} /$ Pentanes. 


\section{Substrate Characterization}

\section{cinnamyl(4-methoxyphenyl)carbamic chloride (1a)}<smiles>COc1ccc(N(C/C=C/c2ccccc2)C(=O)Cl)cc1</smiles>

Prepared according to GP1a on a $7 \mathrm{mmol}$ scale. Isolated by flash column chromatography, $20 \% \rightarrow 50 \% \mathrm{DCM} /$ Pentanes. Isolated $1.26 \mathrm{~g}$ of a white solid (60\% yield).

${ }^{1} \mathbf{H}$ NMR $\left(500 \mathrm{MHz}, \mathrm{CDCl}_{3}\right) \delta 7.39-7.23(\mathrm{~m}, 5 \mathrm{H}), 7.19-7.06(\mathrm{~m}, 2 \mathrm{H}), 6.91$ $(\mathrm{d}, J=8.9 \mathrm{~Hz}, 2 \mathrm{H}), 6.46(\mathrm{~d}, J=15.7 \mathrm{~Hz}, 1 \mathrm{H}), 6.27(\mathrm{dt}, J=15.7,6.9 \mathrm{~Hz}, 1 \mathrm{H})$, $4.45-4.40(\mathrm{~m}, 2 \mathrm{H}), 3.82(\mathrm{~s}, 3 \mathrm{H})$.

${ }^{13}$ C NMR (126 MHz, $\left.\mathrm{CDCl}_{3}\right) \delta 159.5,149.7,136.2,134.9,134.6,129.5$, $128.7,128.1,126.6,122.2,114.6,55.5,55.3$.

IR $\left(\right.$ ATR, $\left.\mathrm{cm}^{-1}\right)$ 1726, 1512, 1446, 1387, 1226, 1039, 977, 874, 838, 720.

HRMS (DART) m/z: $[\mathrm{M}+\mathrm{H}]^{+}$Calcd for $\mathrm{C}_{17} \mathrm{H}_{17} \mathrm{NO}_{2} \mathrm{Cl} 302.0942$; found 302.0942 .

MP $47-50{ }^{\circ} \mathrm{C}$

\section{(E)-(4-methoxyphenyl)(3-(4-methoxyphenyl)allyl)carbamic chloride (1b)}<smiles>COc1ccc(/C=C/CN(C(=O)Cl)c2ccc(OC)cc2)cc1</smiles>

Prepared according to GP1a on a $1.4 \mathrm{mmol}$ scale. Isolated by flash column chromatography, $30 \% \rightarrow 80 \%$ DCM/Pentanes. Isolated $288 \mathrm{mg}$ of a yellow oil ( $68 \%$ yield). ${ }^{1} \mathbf{H}$ NMR $\left(500 \mathrm{MHz}, \mathrm{CDCl}_{3}\right) \delta 7.31-7.22(\mathrm{~m}, 2 \mathrm{H}), 7.17-7.07(\mathrm{~m}$, $2 \mathrm{H}), 6.95-6.86(\mathrm{~m}, 2 \mathrm{H}), 6.88-6.82(\mathrm{~m}, 2 \mathrm{H}), 6.38(\mathrm{~d}, J=15.7 \mathrm{~Hz}$, $1 \mathrm{H}), 6.12(\mathrm{dt}, J=15.7,7.0 \mathrm{~Hz}, 1 \mathrm{H}), 4.55-4.31(\mathrm{~m}, 2 \mathrm{H}), 3.82(\mathrm{~s}, 3 \mathrm{H})$, $3.81(\mathrm{~s}, 3 \mathrm{H})$.

$128.9,127.8,119.8,114.5,114.0,55.5,55.3$. ${ }^{13}$ C NMR $\left(126 \mathrm{MHz}, \mathrm{CDCl}_{3}\right) \delta 159.6,149.6,134.6,134.5,129.5$, IR $\left(\right.$ ATR, $\left.\mathrm{cm}^{-1}\right) 1735,1610,1516,1378,1253,1182,1035,972,843,593$

HRMS (DART) m/z: [M+H] $]^{+}$Calcd for $\mathrm{C}_{18} \mathrm{H}_{19} \mathrm{NO}_{3} \mathrm{Cl} 332.1048$; found 332.1044

\section{(E)-(4-methoxyphenyl)(3-(4-(trifluoromethyl)phenyl)allyl)carbamic chloride (1c)}<smiles>COc1ccc(N(C/C=C/c2ccc(C(F)(F)F)cc2)C(=O)Cl)cc1</smiles>

Prepared according to GP1a on a $4.9 \mathrm{mmol}$ scale. Isolated by flash column chromatography, $40 \% \rightarrow 60 \% \mathrm{DCM} /$ Pentanes. Isolated $968 \mathrm{mg}$ of a white solid ( $37 \%$ yield).

${ }^{1} \mathbf{H}$ NMR $\left(500 \mathrm{MHz}, \mathrm{CDCl}_{3}\right) \delta 7.57(\mathrm{~d}, J=8.1 \mathrm{~Hz}, 2 \mathrm{H}), 7.44(\mathrm{~d}, J=8.1$ $\mathrm{Hz}, 2 \mathrm{H}), 7.18-7.10(\mathrm{~m}, 2 \mathrm{H}), 6.96-6.87(\mathrm{~m}, 2 \mathrm{H}), 6.49(\mathrm{~d}, J=15.8 \mathrm{~Hz}$, $1 \mathrm{H}), 6.36(\mathrm{dt}, J=15.8,6.8 \mathrm{~Hz}, 1 \mathrm{H}), 4.52-4.40(\mathrm{~m}, 2 \mathrm{H}), 3.82(\mathrm{~s}, 3 \mathrm{H})$.

${ }^{13}$ C NMR (126 MHz, $\left.\mathrm{CDCl}_{3}\right) \delta 159.6,149.8,139.6,134.5,133.4,129.9$

$271.9 \mathrm{~Hz}), 114.7,55.5,55.0$. $(\mathrm{q}, J=32.5 \mathrm{~Hz}), 129.4,126.7,125.6(\mathrm{q}, J=3.8 \mathrm{~Hz}), 125.0,124.0(\mathrm{q}, J=$

${ }^{19} \mathbf{F}$ NMR $\left(377 \mathrm{MHz}, \mathrm{CDCl}_{3}\right) \delta-62.55$.

IR $\left(\mathrm{ATR}, \mathrm{cm}^{-1}\right)$ 1735, 1516, 1338, 1244, 1133, 1070, 977, 834, 827, 589

HRMS (DART) m/z: $[\mathrm{M}+\mathrm{H}]^{+}$Calcd for $\mathrm{C}_{18} \mathrm{H}_{16} \mathrm{NO}_{2} \mathrm{~F}_{3} \mathrm{Cl} 370.0816$; found 370.0817 .

MP $54-55^{\circ} \mathrm{C}$

(E)-(3-(4-fluorophenyl)allyl)(4-methoxyphenyl)carbamic chloride (1d) 
<smiles>COc1ccc(N(C/C=C/c2ccc(F)cc2)C(=O)Cl)cc1</smiles>

114.6, 55.5, 55.2.

Prepared according to GP1a on a $2.6 \mathrm{mmol}$ scale. Isolated by flash column chromatography, $20 \% \rightarrow 60 \% \mathrm{DCM} /$ Pentanes. Isolated $103 \mathrm{mg}$ of a clear oil ( $12 \%$ yield).

${ }^{1} \mathbf{H}$ NMR $\left(500 \mathrm{MHz}, \mathrm{CDCl}_{3}\right) \delta 7.34-7.28(\mathrm{~m}, 2 \mathrm{H}), 7.15-7.09(\mathrm{~m}, 2 \mathrm{H})$, $7.07-6.97(\mathrm{~m}, 2 \mathrm{H}), 6.95-6.85(\mathrm{~m}, 2 \mathrm{H}), 6.41(\mathrm{~d}, J=15.8 \mathrm{~Hz}, 1 \mathrm{H}), 6.17$ (dt, $J=15.8,6.9 \mathrm{~Hz}, 1 \mathrm{H}), 4.51-4.30(\mathrm{~m}, 2 \mathrm{H}), 3.82$ (s, 3H).

${ }^{13}$ C NMR $\delta 162.6(\mathrm{~d}, J=247.7 \mathrm{~Hz}), 159.5,149.7,134.6,133.7,132.3(\mathrm{~d}, J$ $=3.3 \mathrm{~Hz}), 129.4,128.1(\mathrm{~d}, J=8.1 \mathrm{~Hz}), 121.9,115.6(\mathrm{~d}, J=21.6 \mathrm{~Hz})$,

${ }^{19}$ F NMR $\left(377 \mathrm{MHz}, \mathrm{CDCl}_{3}\right) \delta-113.56$.

IR $\left(\right.$ ATR, $\left.\mathrm{cm}^{-1}\right) 1730,1601,1512,1391,1222,1106,1021,972,843,620$

HRMS (DART) m/z: [M+H] ${ }^{+}$Calcd for $\mathrm{C}_{17} \mathrm{H}_{16} \mathrm{NO}_{2} \mathrm{FCl} 320.0848$; found 320.0845

\section{(E)-(3-(4-chlorophenyl)allyl)(4-methoxyphenyl)carbamic chloride (1e)}<smiles>COc1ccc(N(C/C=C/c2ccc(Cl)cc2)C(=O)Cl)cc1</smiles>

Prepared according to GP1a on a $2.2 \mathrm{mmol}$ scale. Isolated by flash column chromatography, $20 \% \rightarrow 60 \% \mathrm{DCM} /$ Pentanes. Isolated $181 \mathrm{mg}$ of a yellow oil ( $21 \%$ yield).

${ }^{1} \mathbf{H}$ NMR $\left(500 \mathrm{MHz}, \mathrm{CDCl}_{3}\right) \delta 7.41-7.21(\mathrm{~m}, 4 \mathrm{H}), 7.17-7.09(\mathrm{~m}, 2 \mathrm{H})$, $6.97-6.82(\mathrm{~m}, 2 \mathrm{H}), 6.40(\mathrm{~d}, J=15.8 \mathrm{~Hz}, 1 \mathrm{H}), 6.23(\mathrm{dt}, J=15.8,6.9 \mathrm{~Hz}$, $1 \mathrm{H}), 4.48-4.33(\mathrm{~m}, 2 \mathrm{H}), 3.82(\mathrm{~s}, 3 \mathrm{H})$.

${ }^{13}$ C NMR $\left(126 \mathrm{MHz}, \mathrm{CDCl}_{3}\right) \delta 159.5,149.7,134.6,134.5,133.8,133.6$, $129.4,128.8,127.8,122.8,114.6,55.5,55.1$.

IR $\left(\right.$ ATR, $\left.\mathrm{cm}^{-1}\right) 1739,1512,1378,1249,1097,1021,968,847,771,607$

HRMS (DART) m/z: $[\mathrm{M}+\mathrm{H}]^{+}$Calcd for $\mathrm{C}_{17} \mathrm{H}_{16} \mathrm{NO}_{2} \mathrm{Cl}_{2}$ 336.0553; found 336.0558

\section{(E)-(3-(4-bromophenyl)allyl)(4-methoxyphenyl)carbamic chloride (1f)}<smiles>COc1ccc(N(C/C=C/c2ccc(Br)cc2)C(=O)Cl)cc1</smiles>

Prepared according to GP1a on a $6.6 \mathrm{mmol}$ scale. Isolated by flash column chromatography, $30 \% \rightarrow 60 \% \mathrm{DCM} /$ Pentanes. Isolated $1.72 \mathrm{mg}$ of a white solid $(74 \%$ yield $)$.

${ }^{1} \mathbf{H}$ NMR $\left(500 \mathrm{MHz}, \mathrm{CDCl}_{3}\right) \delta 7.46-7.40(\mathrm{~m}, 2 \mathrm{H}), 7.23-7.17(\mathrm{~m}, 2 \mathrm{H})$, $7.16-7.09(\mathrm{~m}, 2 \mathrm{H}), 6.94-6.87(\mathrm{~m}, 2 \mathrm{H}), 6.39(\mathrm{~d}, J=15.8 \mathrm{~Hz}, 1 \mathrm{H}), 6.25$ (dt, $J=15.8,6.8 \mathrm{~Hz}, 1 \mathrm{H}), 4.42-4.37(\mathrm{~m}, 2 \mathrm{H}), 3.81(\mathrm{~s}, 3 \mathrm{H})$.

${ }^{13} \mathbf{C}$ NMR $\left(126 \mathrm{MHz}, \mathrm{CDCl}_{3}\right) \delta 159.5,149.7,135.10,134.5,133.7,131.8$, $129.4,128.1,123.0,122.0,114.6,55.5,55.2$.

IR $\left(\right.$ ATR, $\left.\mathrm{cm}^{-1}\right)$ 1726, 1516, 1373, 1253, 1173, 1075, 977, 825, 792, 609

HRMS (DART) $\mathrm{m} / \mathrm{z}$ : $[\mathrm{M}+\mathrm{H}]^{+}$Calcd for $\mathrm{C}_{17} \mathrm{H}_{16} \mathrm{NO}_{2} \mathrm{ClBr} 380.0048$; found 380.0051

MP $79-80{ }^{\circ} \mathrm{C}$

\section{(E)-(4-methoxyphenyl)(3-(3-methoxyphenyl)allyl)carbamic chloride (1g)}<smiles>COc1ccc(N(C/C=C/c2cccc(OC)c2)C(=O)Cl)cc1</smiles>

Prepared according to GP1a on a $4.9 \mathrm{mmol}$ scale. Isolated by flash column chromatography, $20 \% \rightarrow 60 \%$ DCM/Pentanes. Isolated $390 \mathrm{mg}$ of a yellow oil ( $24 \%$ yield).

${ }^{1} \mathbf{H}$ NMR $\left(500 \mathrm{MHz}, \mathrm{CDCl}_{3}\right) \delta 7.23(\mathrm{t}, J=7.9 \mathrm{~Hz}, 1 \mathrm{H}), 7.18-7.09(\mathrm{~m}, 2 \mathrm{H})$, $6.97-6.86$ (m, 4H), 6.82 (ddd, $J=8.3,2.6,0.9 \mathrm{~Hz}, 1 \mathrm{H}), 6.42$ (d, $J=15.8 \mathrm{~Hz}$, $1 \mathrm{H}), 6.26(\mathrm{dt}, J=15.8,6.9 \mathrm{~Hz}, 1 \mathrm{H}), 4.65-4.34(\mathrm{~m}, 2 \mathrm{H}), 3.82(\mathrm{~s}, 3 \mathrm{H}), 3.81(\mathrm{~s}$, $3 \mathrm{H})$.

${ }^{13}$ C NMR $\left(126 \mathrm{MHz}, \mathrm{CDCl}_{3}\right) \delta 159.8,159.5,149.7,137.6,134.8,134.6$,

$129.6,129.5,122.5,119.2,114.6,113.8,111.9,55.5,55.3$. 
IR $\left(\right.$ ATR, $\left.\mathrm{cm}^{-1}\right)$ 1726, 1596, 1516, 1396, 1249, 1151, 1044, 985, 843, 771

HRMS (DART) m/z: [M+H] ${ }^{+}$Calcd for $\mathrm{C}_{18} \mathrm{H}_{19} \mathrm{NO}_{3} \mathrm{Cl} 332.1048$; found 332.1046

\section{(E)-(3-(3-fluorophenyl)allyl)(4-methoxyphenyl)carbamic chloride (1h)}<smiles>COc1ccc(N(C/C=C/c2cccc(F)c2)C(=O)Cl)cc1</smiles>

Prepared according to GP1a on a $3.9 \mathrm{mmol}$ scale. Isolated by flash column chromatography, $30 \% \rightarrow 60 \%$ DCM/Pentanes. Isolated $206 \mathrm{mg}$ of a yellow oil (64\% yield).

${ }^{1} \mathbf{H}$ NMR $\left(500 \mathrm{MHz}, \mathrm{CDCl}_{3}\right) \delta 7.30-7.23(\mathrm{~m}, 1 \mathrm{H}), 7.16-7.09(\mathrm{~m}, 3 \mathrm{H}), 7.07$ $-7.01(\mathrm{~m}, 1 \mathrm{H}), 6.99-6.93(\mathrm{~m}, 1 \mathrm{H}), 6.93-6.85(\mathrm{~m}, 2 \mathrm{H}), 6.42(\mathrm{~d}, J=15.7 \mathrm{~Hz}$, $1 \mathrm{H}), 6.26(\mathrm{dt}, J=15.7,6.8 \mathrm{~Hz}, 1 \mathrm{H}), 4.59-4.36(\mathrm{~m}, 2 \mathrm{H}), 3.82(\mathrm{~s}, 3 \mathrm{H})$.

${ }^{13}$ C NMR $\left(126 \mathrm{MHz}, \mathrm{CDCl}_{3}\right) \delta 163.0(\mathrm{~d}, J=245.7 \mathrm{~Hz}), 159.5,149.7,138.5(\mathrm{~d}$, $J=7.6 \mathrm{~Hz}), 134.5,133.7(\mathrm{~d}, J=2.5 \mathrm{~Hz}), 130.1$ (d, $J=8.4 \mathrm{~Hz}), 129.4,123.6$,

$122.5(\mathrm{~d}, J=2.8 \mathrm{~Hz}), 114.9(\mathrm{~d}, J=21.4 \mathrm{~Hz}), 114.7,113.0(\mathrm{~d}, J=21.9 \mathrm{~Hz}), 55.5,55.1$.

${ }^{19}$ F NMR $\left(377 \mathrm{MHz}, \mathrm{CDCl}_{3}\right) \delta-113.22$.

IR $\left(\mathrm{ATR}, \mathrm{cm}^{-1}\right)$ 1735, 1587, 1516, 1378, 1249, 1039, 950, 838, 776, 674

HRMS (DART) m/z: [M+H] $]^{+}$Calcd for $\mathrm{C}_{17} \mathrm{H}_{16} \mathrm{NO}_{2} \mathrm{ClF} 320.0848$; found 320.0849

\section{(E)-(3-(3-chlorophenyl)allyl)(4-methoxyphenyl)carbamic chloride (1i)}<smiles>COc1ccc(N(C/C=C/c2cccc(Cl)c2)C(=O)Cl)cc1</smiles>

Prepared according to GP1a on a $4.1 \mathrm{mmol}$ scale. Isolated by flash column chromatography, $40 \% \rightarrow 60 \% \mathrm{DCM} /$ Pentanes. Isolated $700 \mathrm{mg}$ of a white solid ( $51 \%$ yield).

${ }^{1} \mathbf{H}$ NMR $\left(500 \mathrm{MHz}, \mathrm{CDCl}_{3}\right) \delta 7.33(\mathrm{~d}, J=2.0 \mathrm{~Hz}, 1 \mathrm{H}), 7.29-7.17(\mathrm{~m}, 3 \mathrm{H})$, $7.17-7.07(\mathrm{~m}, 2 \mathrm{H}), 6.98-6.75(\mathrm{~m}, 2 \mathrm{H}), 6.39(\mathrm{~d}, J=15.8 \mathrm{~Hz}, 1 \mathrm{H}), 6.27$ (dt, $J$ $=15.8,6.8 \mathrm{~Hz}, 1 \mathrm{H}), 4.61-4.27(\mathrm{~m}, 2 \mathrm{H}), 3.82(\mathrm{~s}, 3 \mathrm{H})$.

${ }^{13} \mathbf{C}$ NMR $\left(126 \mathrm{MHz}, \mathrm{CDCl}_{3}\right) \delta 159.5,149.7,138.0,134.6,134.5,133.5$, $129.9,129.4,128.0,126.5,124.8,123.8,114.7,55.5,55.1$.

IR $\left(\right.$ ATR, $\left.\mathrm{cm}^{-1}\right) 1735,1507,1440,1387,1222,963,843,789,673,589$

HRMS (DART) m/z: $[\mathrm{M}+\mathrm{H}]^{+}$Calcd for $\mathrm{C}_{17} \mathrm{H}_{16} \mathrm{NO}_{2} \mathrm{Cl}_{2} 336.0553$; found 336.0559 MP $71-72{ }^{\circ} \mathrm{C}$

\section{(E)-(3-(3-bromophenyl)allyl)(4-methoxyphenyl)carbamic chloride (1j)}<smiles>COc1ccc(N(C/C=C/c2cccc(Br)c2)C(=O)Cl)cc1</smiles>

Prepared according to GP1a on a $2.6 \mathrm{mmol}$ scale. Isolated by flash column chromatography, $30 \% \rightarrow 60 \%$ DCM/Pentanes. Isolated $588 \mathrm{mg}$ of a yellow oil (59\% yield).

${ }^{1}$ H NMR $(500 \mathrm{MHz}$, Chloroform- $d$ ) $\delta 7.52-7.45(\mathrm{~m}, 1 \mathrm{H}), 7.43-7.35(\mathrm{~m}$, 1H), $7.27-7.23$ (m, 1H), $7.23-7.16(\mathrm{~m}, 1 \mathrm{H}), 7.15-7.09$ (m, 2H), $6.95-6.82$ $(\mathrm{m}, 2 \mathrm{H}), 6.38(\mathrm{~d}, J=15.8 \mathrm{~Hz}, 1 \mathrm{H}), 6.26(\mathrm{dt}, J=15.8,6.7 \mathrm{~Hz}, 1 \mathrm{H}), 4.62-4.33$ (m, 2H), $3.82(\mathrm{~s}, 3 \mathrm{H})$.

${ }^{13}$ C NMR $\left(126 \mathrm{MHz}, \mathrm{CDCl}_{3}\right) \delta 159.5,149.7,138.3,134.5,133.4,131.0$,

$130.2,129.4,129.4,125.2,123.8,122.8,114.7,55.5,55.1$.

IR $\left(\mathrm{ATR}, \mathrm{cm}^{-1}\right) 1730,1512,1382,1302,1240,1035,972,838,776,669$

HRMS (DART) $\mathrm{m} / \mathrm{z}$ : $[\mathrm{M}+\mathrm{H}]^{+}$Calcd for $\mathrm{C}_{17} \mathrm{H}_{16} \mathrm{NO}_{2} \mathrm{BrCl} 380.0048$; found 380.0046

(E)-(4-methoxyphenyl)(3-(2-methoxyphenyl)allyl)carbamic chloride (1k) 
<smiles>COc1ccc(N(C/C=C/c2ccccc2OC)C(=O)Cl)cc1</smiles>

Prepared according to GP1a on a $5 \mathrm{mmol}$ scale. Isolated by flash column chromatography, $40 \% \rightarrow 70 \% \mathrm{DCM} /$ Pentanes. Isolated $754 \mathrm{mg}$ of a white solid ( $44 \%$ yield).

${ }^{1} \mathbf{H}$ NMR $\left(500 \mathrm{MHz}, \mathrm{CDCl}_{3}\right) \delta 7.39(\mathrm{dd}, J=7.6,1.7 \mathrm{~Hz}, 1 \mathrm{H}), 7.29-7.17(\mathrm{~m}$, 1H), $7.20-7.05(\mathrm{~m}, 2 \mathrm{H}), 6.99-6.80(\mathrm{~m}, 4 \mathrm{H}), 6.76(\mathrm{~d}, J=15.9 \mathrm{~Hz}, 1 \mathrm{H}), 6.27$ (dt, $J=15.9,6.9 \mathrm{~Hz}, 1 \mathrm{H}), 4.62-4.35(\mathrm{~m}, 2 \mathrm{H}), 3.82(\mathrm{~s}, 3 \mathrm{H}), 3.81(\mathrm{~s}, 3 \mathrm{H})$. ${ }^{13}$ C NMR $\left(126 \mathrm{MHz}, \mathrm{CDCl}_{3}\right) \delta 159.4,156.8,149.6,134.6,129.8,129.6$, $129.1,127.1,125.2,122.8,120.7,114.6,110.9,55.7,55.5$.

IR $\left(\right.$ ATR, $\left.\mathrm{cm}^{-1}\right) 1730,1605,1512,1485,1472,1378,1249,1026,981,838$

HRMS (DART) m/z: [M+H] $]^{+}$Calcd for $\mathrm{C}_{18} \mathrm{H}_{19} \mathrm{NO}_{3} \mathrm{Cl} 332.1048$; found 332.1044 MP $41-42{ }^{\circ} \mathrm{C}$

(E)-(3-(3-chlorophenyl)allyl)(4-methoxyphenyl)carbamic chloride (11)<smiles>COc1ccc(N(C/C=C/c2ccco2)C(=O)Cl)cc1</smiles>

Prepared according to GP1a on a $7 \mathrm{mmol}$ scale. Isolated by flash column chromatography, $30 \% \rightarrow 80 \% \mathrm{DCM} /$ Pentanes. Isolated $1.05 \mathrm{~g}$ of a brown oil ( $57 \%$ yield).

${ }^{1} \mathbf{H}$ NMR $\left(500 \mathrm{MHz}, \mathrm{CDCl}_{3}\right) \delta 7.38-7.31(\mathrm{~m}, 1 \mathrm{H}), 7.23-7.08(\mathrm{~m}, 2 \mathrm{H}), 6.95-$ $6.86(\mathrm{~m}, 2 \mathrm{H}), 6.36(\mathrm{dd}, J=3.3,1.9 \mathrm{~Hz}, 1 \mathrm{H}), 6.31-6.12(\mathrm{~m}, 3 \mathrm{H}), 4.55-4.30$ $(\mathrm{m}, 2 \mathrm{H}), 3.80(\mathrm{~s}, 4 \mathrm{H})$.

${ }^{13}$ C NMR $\left(126 \mathrm{MHz}, \mathrm{CDCl}_{3}\right) \delta 159.5,151.7,149.6,142.4,134.6,129.4,123.0$, $120.7,114.6,111.4,108.9,55.6,55.0$.

IR $\left(\right.$ ATR, $\left.\mathrm{cm}^{-1}\right) 1735,1509,1387,1244,1021,972,843,745,584$

HRMS (DART) m/z: $[\mathrm{M}+\mathrm{H}]^{+}$Calcd for $\mathrm{C}_{15} \mathrm{H}_{15} \mathrm{NO}_{3} \mathrm{Cl} 292.0735$; found 292.0738

\section{(E)-(4-methoxyphenyl)(3-(thiophen-2-yl)allyl)carbamic chloride (1m)}<smiles>COc1ccc(N(C/C=C/c2cccs2)C(=O)Cl)cc1</smiles>

Prepared according to GP1a on a $3.5 \mathrm{mmol}$ scale. Isolated by flash column chromatography, $30 \% \rightarrow 80 \% \mathrm{DCM} /$ Pentanes. Isolated $442 \mathrm{mg}$ of a yellow oil ( $41 \%$ yield).

${ }^{1} \mathbf{H}$ NMR $\left(500 \mathrm{MHz}, \mathrm{CDCl}_{3}\right) \delta 7.20-7.16(\mathrm{~m}, 1 \mathrm{H}), 7.15-7.09(\mathrm{~m}, 2 \mathrm{H}), 6.98-$ $6.93(\mathrm{~m}, 2 \mathrm{H}), 6.93-6.89(\mathrm{~m}, 2 \mathrm{H}), 6.58(\mathrm{~d}, J=15.6 \mathrm{~Hz}, 1 \mathrm{H}), 6.08(\mathrm{dt}, J=15.6$, $7.0 \mathrm{~Hz}, 1 \mathrm{H}), 4.46-4.33(\mathrm{~m}, 2 \mathrm{H}), 3.82(\mathrm{~s}, 3 \mathrm{H})$.

${ }^{13} \mathrm{C}$ NMR $\left(126 \mathrm{MHz}, \mathrm{CDCl}_{3}\right) \delta 159.50,149.64,141.09,134.52,129.48$, $128.05,127.45,126.50,124.93,121.58,114.63,55.48,55.09$.

IR $\left(\right.$ ATR, $\left.\mathrm{cm}^{-1}\right)$ 1734, 1514, 1382, 1309, 1217, 1039, 966, 847, 797, 705

HRMS (DART) m/z: [M+H] ${ }^{+}$Calcd for $\mathrm{C}_{15} \mathrm{H}_{15} \mathrm{NO}_{2} \mathrm{SCl} 308.0507$; found 308.0500

cinnamyl(phenyl)carbamic chloride (1n)<smiles>O=C(Cl)N(C/C=C/c1ccccc1)c1ccccc1</smiles>

Prepared according to GP1a on a $10 \mathrm{mmol}$ scale. Isolated by flash column chromatography, $20 \% \rightarrow 70 \% \mathrm{DCM} /$ Pentanes. Isolated $1.50 \mathrm{~g}$ of a white solid (55\% yield).

${ }^{1} \mathbf{H}$ NMR $\left(500 \mathrm{MHz}, \mathrm{CDCl}_{3}\right) \delta 7.62-7.12(\mathrm{~m}, 10 \mathrm{H}), 6.48(\mathrm{~d}, J=15.8 \mathrm{~Hz}$, $1 \mathrm{H}), 6.29$ (dt, $J=15.8,6.9 \mathrm{~Hz}, 1 \mathrm{H}), 4.47$ (d, $J=6.9 \mathrm{~Hz}, 2 \mathrm{H})$.

${ }^{13}$ C NMR $\left(126 \mathrm{MHz}, \mathrm{CDCl}_{3}\right) \delta 149.2,141.8,136.2,135.0,129.6,128.7,128.5$, $128.3,128.2,126.6,122.1,55.2$.

IR $\left(\mathrm{ATR}, \mathrm{cm}^{-1}\right) 1735,1503,1387,1235,1070,972,878,767,705,589$

HRMS (DART) m/z: [M+H] $]^{+}$Calcd for $\mathrm{C}_{16} \mathrm{H}_{15} \mathrm{NOCl} 272.0837$; found 272.0830 MP $86-88^{\circ} \mathrm{C}$ 
cinnamyl(p-tolyl)carbamic chloride (10)<smiles>Cc1ccc(N(C/C=C/c2ccccc2)C(=O)Cl)cc1</smiles>

Prepared according to GP1a on a $10 \mathrm{mmol}$ scale. Isolated by flash column chromatography, $30 \% \rightarrow 80 \% \mathrm{DCM} /$ Pentanes. Isolated $500 \mathrm{mg}$ of an off-white solid (18\% yield).

${ }^{1} \mathbf{H}$ NMR $\left(500 \mathrm{MHz}, \mathrm{CDCl}_{3}\right) \delta 7.40-7.30(\mathrm{~m}, 4 \mathrm{H}), 7.30-7.25(\mathrm{~m}, 1 \mathrm{H}), 7.25$ $-7.18(\mathrm{~m}, 2 \mathrm{H}), 7.18-7.08(\mathrm{~m}, 2 \mathrm{H}), 6.47(\mathrm{~d}, J=15.8 \mathrm{~Hz}, 1 \mathrm{H}), 6.28(\mathrm{dt}, J=$ $15.8,6.9 \mathrm{~Hz}, 1 \mathrm{H}), 4.44(\mathrm{~d}, J=6.9 \mathrm{~Hz}, 2 \mathrm{H}), 2.39(\mathrm{~s}, 3 \mathrm{H})$.

${ }^{13}$ C NMR $\left(126 \mathrm{MHz}, \mathrm{CDCl}_{3}\right) \delta 149.4,139.2,138.7,136.2,134.9,130.2$, $128.6,128.3,128.1,128.1,126.6,122.2,55.3,21.2$.

IR $\left(\right.$ ATR, $\left.\mathrm{cm}^{-1}\right)$ 1739, 1507, 1387, 1235, 1204, 972, 821, 763, 678, 589

HRMS (DART) m/z: [M+H] ${ }^{+}$Calcd for $\mathrm{C}_{17} \mathrm{H}_{17} \mathrm{NOCl} 286.0993$; found 286.0992

MP $51-52{ }^{\circ} \mathrm{C}$

cinnamyl(4-methoxybenzyl)carbamic chloride (1p)<smiles>COc1ccc(CN(C/C=C/c2ccccc2)C(=O)Cl)cc1</smiles>

Prepared according to GP1b on a $5 \mathrm{mmol}$ scale. Isolated by flash column chromatography, $2 \% \rightarrow 10 \%$ EtOAc/Pentanes. Isolated $540 \mathrm{mg}$ of a yellow oil (34\% yield). Product was isolated as a 1.2:1 mixture of rotamers. ${ }^{1} H$ NMR data is listed for the major rotamer. ${ }^{13} \mathrm{C} N M R$ data is listed for a mixture of the rotamers.

${ }^{1} \mathbf{H}$ NMR $\left(500 \mathrm{MHz}, \mathrm{CDCl}_{3}\right) \delta 7.41-7.21(\mathrm{~m}, 7 \mathrm{H}), 6.96-6.86(\mathrm{~m}$, $2 \mathrm{H}), 6.47(\mathrm{~d}, J=6.7 \mathrm{~Hz}, 1 \mathrm{H}), 6.17-6.06(\mathrm{~m}, 1 \mathrm{H}), 4.56(\mathrm{~s}, 2 \mathrm{H}), 4.16$

$(\mathrm{d}, J=5.4 \mathrm{~Hz}, 2 \mathrm{H}), 3.81(\mathrm{~s}, 3 \mathrm{H})$.

${ }^{13}$ C NMR $\left(126 \mathrm{MHz}, \mathrm{CDCl}_{3}\right) \delta 159.53,159.50,150.09,149.52,136.03,135.97,134.56,133.70,129.89$, $128.82,128.70,128.66,128.17,128.14,127.61,127.24,126.54,126.53,122.58,122.24,114.32,114.25$, $55.34,55.32,52.85,51.76,51.26,50.29$.

IR $\left(\mathrm{ATR}, \mathrm{cm}^{-1}\right) 1724,1615,1532,1450,1404,1249,1186,1039,989,751$

HRMS (DART) m/z: [M+H] $]^{+}$Calcd for $\mathrm{C}_{18} \mathrm{H}_{19} \mathrm{NO}_{2} \mathrm{Cl} 316.1098$; found 316.1091

(E)-(4-methoxyphenyl)(4-phenylbut-3-en-2-yl)carbamic chloride (1q)<smiles>COc1ccc(N(C(=O)Cl)/C(C)=C/c2ccccc2)cc1</smiles>

Prepared according to GP1c on a $36 \mathrm{mmol}$ scale. Isolated by flash column chromatography, $10 \% \rightarrow 40 \%$ DCM/Pentanes. Isolated $3.04 \mathrm{~g}$ of a white solid ( $80 \%$ yield).

${ }^{1}$ H NMR $\left(500 \mathrm{MHz}, \mathrm{CDCl}_{3}\right) \delta 7.37-7.30(\mathrm{~m}, 5 \mathrm{H}), 7.30-7.24(\mathrm{~m}, 1 \mathrm{H}), 7.18$ $-7.00(\mathrm{~m}, 2 \mathrm{H}), 6.98-6.80(\mathrm{~m}, 2 \mathrm{H}), 6.55(\mathrm{dd}, J=15.9,1.2 \mathrm{~Hz}, 1 \mathrm{H}), 6.11$ (dd, $J=15.9,7.4 \mathrm{~Hz}, 1 \mathrm{H}), 5.39-5.10(\mathrm{~m}, 1 \mathrm{H}), 3.83(\mathrm{~s}, 3 \mathrm{H}), 1.35(\mathrm{~d}, J=6.9 \mathrm{~Hz}$, $3 \mathrm{H})$.

${ }^{13}$ C NMR $\left(126 \mathrm{MHz}, \mathrm{CDCl}_{3}\right) \delta 159.7,149.7,136.3,132.6,131.3,131.1$, $128.7,128.1,128.0,126.6,114.2,57.8,55.5,18.6$.

IR $\left(\right.$ ATR, $\left.\mathrm{cm}^{-1}\right)$ 1722, 1624, 1526, 1445, 1312, 1254, 1213, 1035, 990, 919

HRMS (DART) m/z: $[\mathrm{M}+\mathrm{H}]^{+}$Calcd for $\mathrm{C}_{18} \mathrm{H}_{19} \mathrm{NO}_{2} \mathrm{Cl} 316.1099$; found 316.1107 MP $35-37{ }^{\circ} \mathrm{C}$

(4-methoxyphenyl)(3-phenylbut-3-en-1-yl)carbamic chloride (3a) 
<smiles>C=C(CCN(C(=O)Cl)c1ccc(OC)cc1)c1ccccc1</smiles>

Prepared according to GP2a on a $5 \mathrm{mmol}$ scale. Isolated by flash column chromatography, $20 \% \rightarrow 60 \%$ DCM/Pentanes. Isolated $322 \mathrm{mg}(1 \mathrm{mmol})$ of a yellow oil ( $20 \%$ yield).

${ }^{1}$ H NMR $\left(500 \mathrm{MHz}, \mathrm{CDCl}_{3}\right) \delta 7.45-7.20(\mathrm{~m}, 5 \mathrm{H}), 7.15-7.10(\mathrm{~m}, 2 \mathrm{H}), 6.97$ $-6.90(\mathrm{~m}, 2 \mathrm{H}), 5.38(\mathrm{~d}, J=1.1 \mathrm{~Hz}, 1 \mathrm{H}), 5.12(\mathrm{~d}, J=1.1 \mathrm{~Hz}, 1 \mathrm{H}), 4.00-3.66$ $(\mathrm{m}, 5 \mathrm{H}), 2.88-2.81(\mathrm{~m}, 2 \mathrm{H})$.

${ }^{13}$ C NMR $\left(126 \mathrm{MHz}, \mathrm{CDCl}_{3}\right) \delta 159.5,149.5,144.4,140.0,134.7,129.4$, $128.5,127.8,125.9,114.6,114.4,55.5,52.3,33.0$.

IR $\left(\right.$ ATR, $\left.\mathrm{cm}^{-1}\right) 1740,1516,1387,1307,1231,1169,1287,1026,847,714$

HRMS (DART) m/z: [M+H] $]^{+}$Calcd for $\mathrm{C}_{18} \mathrm{H}_{18} \mathrm{NO}_{2} \mathrm{Cl} 316.1099$; found 316.1092

\section{(4-methoxyphenyl)(3-(2-methoxyphenyl)but-3-en-1-yl)carbamic chloride (3b)}<smiles>C=C(CCN(C(=O)Cl)c1ccc(OC)cc1)c1ccccc1OC</smiles>

Prepared according to GP2b on a $6.6 \mathrm{mmol}$ scale. Isolated by flash column chromatography, $30 \% \rightarrow 80 \% \mathrm{DCM} /$ Pentanes. Isolated $383 \mathrm{mg}$ of a clear oil (16\% yield).

${ }^{1}$ H NMR $\left(500 \mathrm{MHz}, \mathrm{CDCl}_{3}\right) \delta 7.30-7.21(\mathrm{~m}, 1 \mathrm{H}), 7.13-7.04(\mathrm{~m}, 3 \mathrm{H}), 6.96$ $-6.86(\mathrm{~m}, 3 \mathrm{H}), 6.83(\mathrm{dd}, J=8.3,1.1 \mathrm{~Hz}, 1 \mathrm{H}), 5.23-5.04(\mathrm{~m}, 2 \mathrm{H}), 3.83(\mathrm{~s}$, $3 \mathrm{H}), 3.79-3.72(\mathrm{~m}, 2 \mathrm{H}), 3.70(\mathrm{~s}, 3 \mathrm{H}), 2.86-2.73(\mathrm{~m}, 2 \mathrm{H})$.

${ }^{13}$ C NMR $\left(126 \mathrm{MHz}, \mathrm{CDCl}_{3}\right) \delta 159.4,156.4,149.3,145.1,134.7,130.7$, $130.1,129.5,128.9,120.6,116.4,114.5,110.6,55.5,55.2,52.3,33.7$.

IR $\left(\right.$ ATR, $\left.\mathrm{cm}^{-1}\right) 1744,1601,1503,1392,1240,1187,1026,928,759,580$

HRMS (DART) m/z: [M+H] $]^{+}$Calcd for $\mathrm{C}_{19} \mathrm{H}_{21} \mathrm{NO}_{3} \mathrm{Cl}$ 346.1203; found 346.1199

\section{(4-methoxyphenyl)(3-(m-tolyl)but-3-en-1-yl)carbamic chloride (3c)}<smiles>C=C(CCN(C(=O)Cl)c1ccc(OC)cc1)c1cccc(C)c1</smiles>

Prepared according to GP2a on a $15 \mathrm{mmol}$ scale. Isolated by flash column chromatography, $20 \% \rightarrow 60 \% \mathrm{DCM} /$ Pentanes. Isolated $1.03 \mathrm{~g}$ of a white solid ( $21 \%$ yield).

${ }^{1} \mathbf{H}$ NMR $\left(500 \mathrm{MHz}, \mathrm{CDCl}_{3}\right) \delta 7.18(\mathrm{td}, J=7.4,0.9 \mathrm{~Hz}, 1 \mathrm{H}), 7.14-7.09(\mathrm{~m}$, $4 \mathrm{H}), 7.09-7.02(\mathrm{~m}, 1 \mathrm{H}), 6.96-6.86(\mathrm{~m}, 2 \mathrm{H}), 5.34(\mathrm{~d}, J=1.2 \mathrm{~Hz}, 1 \mathrm{H}), 5.13$ $-5.00(\mathrm{~m}, 1 \mathrm{H}), 3.84(\mathrm{~s}, 3 \mathrm{H}), 3.83-3.77(\mathrm{~m}, 2 \mathrm{H}), 2.86-2.78(\mathrm{~m}, 2 \mathrm{H}), 2.31$ $(\mathrm{d}, J=0.9 \mathrm{~Hz}, 3 \mathrm{H})$

${ }^{13}$ C NMR $\left(126 \mathrm{MHz}, \mathrm{CDCl}_{3}\right) \delta 159.4,149.5,144.4,139.9,138.0,134.7$,

$129.4,128.5,128.3,126.7,122.9,114.6,114.1,55.5,52.3,33.0,21.4$.

IR $\left(\right.$ ATR, $\left.\mathrm{cm}^{-1}\right) 1717,1512,1387,1320,1236,1669,1044,910,803,736$

HRMS (DART) m/z: $[\mathrm{M}+\mathrm{H}]^{+}$Calcd for $\mathrm{C}_{19} \mathrm{H}_{21} \mathrm{NO}_{2} \mathrm{Cl} 330.1255$; found 330.1257

MP $54-55{ }^{\circ} \mathrm{C}$

(4-methoxyphenyl)(3-(naphthalen-2-yl)but-3-en-1-yl)carbamic chloride (3d)<smiles>C=C(CCN(C(=O)Cl)c1ccc(OC)cc1)c1ccc2ccccc2c1</smiles>

Prepared according to GP2a on a $15 \mathrm{mmol}$ scale. Isolated by flash column chromatography, $20 \% \rightarrow 60 \%$ DCM/Pentanes. Isolated $678 \mathrm{mg}$ of a white solid (12\% yield).

${ }^{1}$ H NMR $\left(500 \mathrm{MHz}, \mathrm{CDCl}_{3}\right) \delta 7.85-7.66(\mathrm{~m}, 4 \mathrm{H}), 7.51(\mathrm{dd}, J=8.5,1.9$ $\mathrm{Hz}, 1 \mathrm{H}), 7.49-7.38(\mathrm{~m}, 2 \mathrm{H}), 7.20-7.09(\mathrm{~m}, 2 \mathrm{H}), 6.98-6.87(\mathrm{~m}, 2 \mathrm{H})$, $5.52(\mathrm{~d}, J=1.1 \mathrm{~Hz}, 1 \mathrm{H}), 5.22(\mathrm{q}, J=1.1 \mathrm{~Hz}, 1 \mathrm{H}), 4.00-3.79(\mathrm{~m}, 5 \mathrm{H}), 3.15$ $-2.85(\mathrm{~m}, 2 \mathrm{H})$.

${ }^{13}$ C NMR $\left(126 \mathrm{MHz}, \mathrm{CDCl}_{3}\right) \delta 159.4,149.5,144.0,136.9,134.7,133.3$, $132.9,129.4,128.2,128.0,127.5,126.2,126.0,124.6,124.1,114.9,114.6,55.5,52.4,33.0$. IR $\left(\mathrm{ATR}, \mathrm{cm}^{-1}\right)$ 1726, 1521, 1387, 1222, 1182, 1044, 856, 763, 651, 575 
HRMS (DART) m/z: [M+H] $]^{+}$Calcd for $\mathrm{C}_{22} \mathrm{H}_{21} \mathrm{NO}_{2} \mathrm{Cl}$ 366.1255; found 366.1249

MP $74-75{ }^{\circ} \mathrm{C}$

(4-methoxyphenyl)(3-(4-methoxyphenyl)but-3-en-1-yl)carbamic chloride (3e)<smiles>C=C(CCN(C(=O)Cl)c1ccc(OC)cc1)c1ccc(OC)cc1</smiles>

Prepared according to GP2a on an $8 \mathrm{mmol}$ scale. Isolated by flash column chromatography, $30 \% \rightarrow 60 \% \mathrm{DCM} /$ Pentanes. Isolated $1.50 \mathrm{~g}$ of a white solid ( $54 \%$ yield).

${ }^{1}$ H NMR $\left(500 \mathrm{MHz}, \mathrm{CDCl}_{3}\right) \delta 7.31-7.25(\mathrm{~m}, 2 \mathrm{H}), 7.15-7.08(\mathrm{~m}$, $2 \mathrm{H}), 6.95-6.88(\mathrm{~m}, 2 \mathrm{H}), 6.86-6.80(\mathrm{~m}, 2 \mathrm{H}), 5.29(\mathrm{~d}, J=1.2 \mathrm{~Hz}, 1 \mathrm{H})$, $5.01(\mathrm{~d}, J=1.2 \mathrm{~Hz}, 1 \mathrm{H}), 3.84(\mathrm{~s}, 3 \mathrm{H}), 3.83-3.76(\mathrm{~m}, 5 \mathrm{H}), 2.83-2.77$ (m, 2H).

${ }^{13} \mathbf{C}$ NMR $\left(126 \mathrm{MHz}, \mathrm{CDCl}_{3}\right) \delta 159.4,159.3,149.5,143.6,134.7$,

$132.3,129.4,127.0,114.6,113.8,112.7,55.5,55.3,52.4,33.1$.

IR $\left(\right.$ ATR, $\left.\mathrm{cm}^{-1}\right) 1731,1606,1512,1391,1253,1187,1120,1026,883,843$

HRMS (DART) m/z: [M+H] $]^{+}$Calcd for $\mathrm{C}_{19} \mathrm{H}_{21} \mathrm{NO}_{3} \mathrm{Cl} 346.1205$; found 346.1204

MP $56-57{ }^{\circ} \mathrm{C}$

(4-methoxyphenyl)(3-(4-(trifluoromethyl)phenyl)but-3-en-1-yl)carbamic chloride (3f)<smiles>C=C(CCN(C(=O)Cl)c1ccc(OC)cc1)c1ccc(C(F)(F)F)cc1</smiles>

Prepared according to GP2 on a $20 \mathrm{mmol}$ scale. Isolated by flash column chromatography, $30 \% \rightarrow 60 \% \mathrm{DCM} /$ Pentanes. Isolated $1.44 \mathrm{~g}$ of a white solid (19\% yield).

${ }^{1} \mathbf{H}$ NMR $\left(500 \mathrm{MHz}, \mathrm{CDCl}_{3}\right) \delta 7.60-7.52(\mathrm{~m}, 2 \mathrm{H}), 7.43(\mathrm{~d}, J=8.1 \mathrm{~Hz}$, $2 \mathrm{H}), 7.15-7.08(\mathrm{~m}, 2 \mathrm{H}), 6.96-6.89(\mathrm{~m}, 2 \mathrm{H}), 5.44(\mathrm{~d}, J=0.8 \mathrm{~Hz}, 1 \mathrm{H})$, $5.23-5.20(\mathrm{~m}, 1 \mathrm{H}), 3.84(\mathrm{~s}, 3 \mathrm{H}), 3.83-3.76(\mathrm{~m}, 2 \mathrm{H}), 2.89-2.80(\mathrm{~m}$, $2 \mathrm{H})$.

${ }^{13}$ C NMR $\left(126 \mathrm{MHz}, \mathrm{CDCl}_{3}\right) \delta 159.5,149.6,143.6,143.3,134.5,129.7$

$(\mathrm{q}, J=32.4 \mathrm{~Hz}), 129.3,126.2,125.4(\mathrm{q}, J=3.7 \mathrm{~Hz}), 124.1(\mathrm{q}, J=271.9 \mathrm{~Hz}), 116.4,114.7,55.5,52.0$, 32.9.

${ }^{19}$ F NMR $\left(377 \mathrm{MHz}, \mathrm{CDCl}_{3}\right) \delta-62.57$.

IR $\left(\right.$ ATR, $\left.\mathrm{cm}^{-1}\right)$ 1735, 1610, 1507, 1325, 1245, 1173, 1110, 914, 823, 576

HRMS (DART) m/z: $[\mathrm{M}+\mathrm{H}]^{+}$Calcd for $\mathrm{C}_{19} \mathrm{H}_{18} \mathrm{NO}_{2} \mathrm{~F}_{3} \mathrm{Cl} 384.0973$; found 384.0979

MP $61-62{ }^{\circ} \mathrm{C}$ 


\section{Catalytic Reaction Protocols}

\section{Catalytic Reaction Protocol - General Procedure 3 (GP3)}<smiles>[R]c1cccc(/C=C/C([R])N(C(=O)Cl)c2cccc([R])c2)c1</smiles>

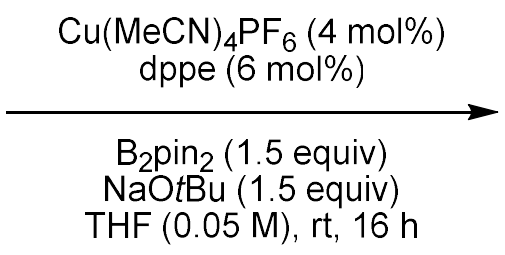

Scheme S6.

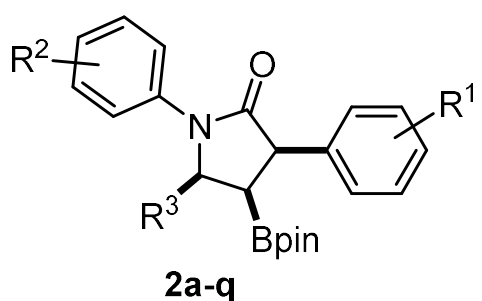

$2 a-q$

GP3 for products 2a-q (Scheme S6): Reactions were performed in oven dried $2 \mathrm{dr}$ vials under argon equipped with a stir bar. Tetrakisacetonitrile copper hexafluorophosphate $(3.0 \mathrm{mg}, 0.008 \mathrm{mmol}, 4 \mathrm{~mol} \%)$, dppe (4.8 mg, $0.012 \mathrm{mmol}, 6 \mathrm{~mol} \%$ ), and sodium tert-butoxide ( $29 \mathrm{mg}, 0.3 \mathrm{mmol}, 1.5$ equiv) were added to a vial along with $1 \mathrm{~mL}$ of anhydrous THF. The solution stirred for 2 minutes, then bis(pinacolato)diboron ( $76 \mathrm{mg}, 0.3 \mathrm{mmol}, 1.5$ equiv) in $1 \mathrm{~mL}$ THF was added. The solution stirrred for 2 minutes before addition of the substrate $(0.2 \mathrm{mmol}, 1.0$ equiv) in $2 \mathrm{~mL}$ of THF was added. The vial was sealed with a teflon septa (ThermoScientific National B7995-15). The reaction was stirred for $16 \mathrm{~h}$ then filtered through a silica pad and concentrated. The crude was purified by silica gel chromatography in ethyl acetate and pentane mixtures. Products were characterized with basified chloroform $\left(\mathrm{K}_{2} \mathrm{CO}_{3}\right)$.

\section{Catalytic Reaction Protocol - General Procedure 4 (GP4)}<smiles>[R]c1ccccc1C(=C)CCN([R16])C(=O)Cl</smiles>

3a-f

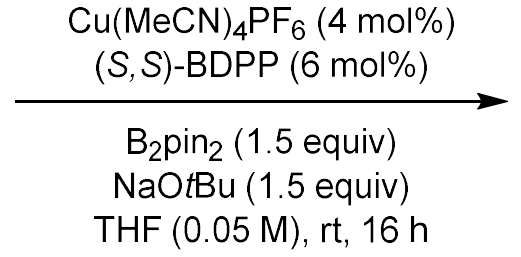

Scheme S7.<smiles>[R]c1ccc([C@]2(CBr)CCN([R16])C2=O)cc1</smiles>

4a-f

GP4 for products 4a-f (Scheme S7): Reactions were performed in oven dried $2 \mathrm{dr}$ vials under argon equipped with a stir bar. Tetrakisacetonitrile copper hexafluorophosphate $(3.0 \mathrm{mg}, 0.008 \mathrm{mmol}, 4 \mathrm{~mol} \%)$, $(S, S)$-BDPP (5.3 mg, $0.012 \mathrm{mmol}, 6 \mathrm{~mol} \%$ ), and sodium tert-butoxide ( $29 \mathrm{mg}, 0.3 \mathrm{mmol}, 1.5$ equiv) were added to the vial along with $1 \mathrm{~mL}$ of anhydrous THF. The solution stirred for 5 minutes prior to addition of bis(pinacolato)diboron ( $76 \mathrm{mg}, 0.3 \mathrm{mmol}, 1.5$ equiv) in $1 \mathrm{~mL}$ THF. The solution stirred for 5 minutes before addition of the substrate $(0.2 \mathrm{mmol}, 1$ equiv) in $2 \mathrm{~mL}$ of THF. The vial was sealed with a teflon septa (ThermoScientific National B7995-15). The reaction was stirred for $16 \mathrm{~h}$ then filtered through a silica pad and concentrated. The crude was purified by silica gel chromatography in ethyl acetate and pentane mixtures. Products were characterized with basified chloroform $\left(\mathrm{K}_{2} \mathrm{CO}_{3}\right)$. 


\section{Preliminary Chiral Ligand Screen for Enantioselective Variant}<smiles>Cc1ccc(N(C/C=C/c2ccccc2)C(=O)Cl)cc1</smiles>

10

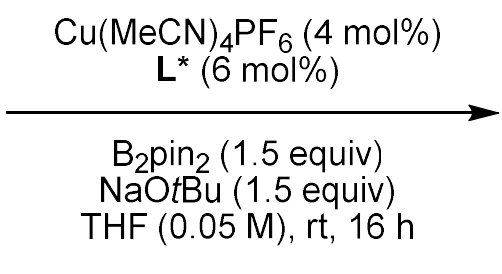

Scheme S8.

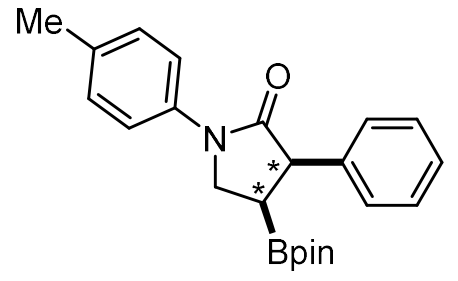

30

3o was synthesized according to GP4, while varying the chiral ligand.

Table S1.

\begin{tabular}{|c|c|c|c|}
\hline Ligand & \% Yield of 3o $^{[\mathbf{a}]}$ & er & dr \\
\hline$(S)$-BINAP & 66 & $50: 50$ & $>20: 1$ \\
\hline$(R)$-SEGPHOS & 44 & $62.5: 37.5$ & $>20: 1$ \\
\hline$(R, R)$-QuinoxP* & 58 & $82: 18$ & $6: 1$ \\
\hline$(S, S)$-DIOP & 33 & $71.5: 28.5$ & $3: 1$ \\
\hline SL-J001-1 & 54 & $83.5: 16.5$ & $>20: 1$ \\
\hline$(R)$ - ProPhos & 93 & $64: 36$ & $>20: 1$ \\
\hline$(R)$-CatASiumD & 88 & $77.5: 22.5$ & \\
\hline
\end{tabular}

[a] Yields determined by NMR and are of the major diastereomer. 


\section{Product Characterization}

1-(4-methoxyphenyl)-3-phenyl-4-(4,4,5,5-tetramethyl-1,3,2-dioxaborolan-2-yl)pyrrolidin-2-one (2a)

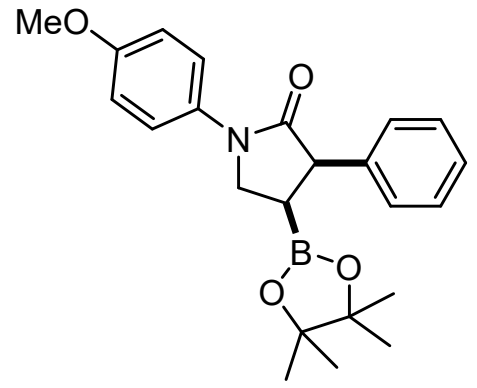

Prepared according to GP3. Isolated by flash column chromatography, $5 \% \rightarrow 25 \%$ EtOAc/Pentanes. Isolated $77 \mathrm{mg}$ of a white solid (97\% yield). ${ }^{1} \mathbf{H}$ NMR $\left(500 \mathrm{MHz}, \mathrm{CDCl}_{3}\right) \delta 7.65(\mathrm{~d}, J=9.1 \mathrm{~Hz}, 2 \mathrm{H}), 7.30-7.24(\mathrm{~m}$, $2 \mathrm{H}), 7.24-7.17(\mathrm{~m}, 3 \mathrm{H}), 6.92(\mathrm{~d}, J=9.1 \mathrm{~Hz}, 2 \mathrm{H}), 4.23(\mathrm{t}, J=10.3 \mathrm{~Hz}$, $1 \mathrm{H}), 3.96(\mathrm{~d}, J=10.3 \mathrm{~Hz}, 1 \mathrm{H}), 3.92(\mathrm{dd}, J=10.3,9.1 \mathrm{~Hz}, 1 \mathrm{H}), 3.81$ (s, $3 \mathrm{H}), 2.42(\mathrm{td}, J=10.3,9.1 \mathrm{~Hz}, 1 \mathrm{H}), 0.95(\mathrm{~s}, 6 \mathrm{H}), 0.94(\mathrm{~s}, 6 \mathrm{H})$

${ }^{13}$ C NMR $\left(126 \mathrm{MHz}, \mathrm{CDCl}_{3}\right) \delta 174.6,156.6,139.6,132.8,128.6,128.4$, $127.2,121.7,114.0,83.7,55.5,51.8,50.2,24.7,24.6$.

IR $\left(\mathrm{ATR}, \mathrm{cm}^{-1}\right)$ 2983, 1681, 1516, 1391, 1333, 1244, 1133, 1035, 825,

696

HRMS (DART) m/z: $[\mathrm{M}+\mathrm{H}]^{+}$Calcd for $\mathrm{C}_{23} \mathrm{H}_{29} \mathrm{BNO}_{4} 394.2184$; found 394.2189

MP $144-146{ }^{\circ} \mathrm{C}$

\section{1,3-bis(4-methoxyphenyl)-4-(4,4,5,5-tetramethyl-1,3,2-dioxaborolan-2-yl)pyrrolidin-2-one (2b)}

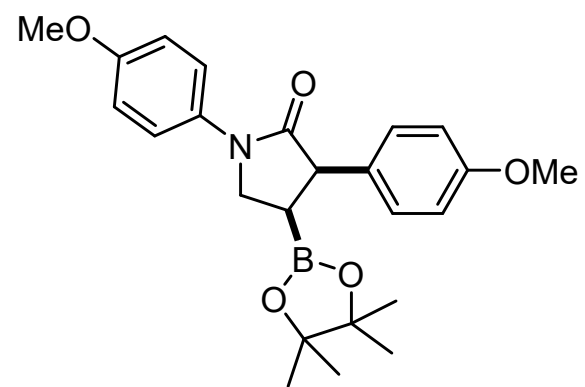

Prepared according to GP3. Isolated by flash column

chromatography, $5 \% \rightarrow 25 \%$ EtOAc/Pentanes. Isolated $66 \mathrm{mg}$ of a white solid (78\% yield).

${ }^{1} \mathbf{H}$ NMR $\left(500 \mathrm{MHz}, \mathrm{CDCl}_{3}\right) \delta 7.64(\mathrm{~d}, J=9.2 \mathrm{~Hz}, 2 \mathrm{H}), 7.14(\mathrm{~d}, J$ $=8.7 \mathrm{~Hz}, 2 \mathrm{H}), 6.91(\mathrm{~d}, J=9.2 \mathrm{~Hz}, 2 \mathrm{H}), 6.81(\mathrm{~d}, J=8.7 \mathrm{~Hz}, 2 \mathrm{H})$, $4.21(\mathrm{t}, J=10.2 \mathrm{~Hz}, 1 \mathrm{H}), 3.94-3.87(\mathrm{~m}, 2 \mathrm{H}), 3.80(\mathrm{~s}, 3 \mathrm{H}), 3.75$ $(\mathrm{s}, 3 \mathrm{H}), 2.39(\mathrm{td}, 10.2,9.11 \mathrm{H}), 0.98(\mathrm{~s}, 6 \mathrm{H}), 0.97(\mathrm{~s}, 6 \mathrm{H})$.

${ }^{13}$ C NMR $\left(126 \mathrm{MHz}, \mathrm{CDCl}_{3}\right) \delta 174.9,158.8,156.5,132.8,131.9$, $129.5,121.7,114.0,114.0,83.7,55.5,51.0,50.1,24.7$.

IR $\left(\right.$ ATR, $\left.\mathrm{cm}^{-1}\right)$ 2975, 1682, 1512, 1392, 1338, 1254, 1187, 1138, 1031, 825

HRMS (DART) $\mathrm{m} / \mathrm{z}$ : $[\mathrm{M}+\mathrm{H}]^{+}$Calcd for $\mathrm{C}_{24} \mathrm{H}_{31} \mathrm{BNO}_{5} 424.2290$; found 424.2293

MP $141-144{ }^{\circ} \mathrm{C}$

1-(4-methoxyphenyl)-4-(4,4,5,5-tetramethyl-1,3,2-dioxaborolan-2-yl)-3-(4(trifluoromethyl)phenyl)pyrrolidin-2-one (2c)

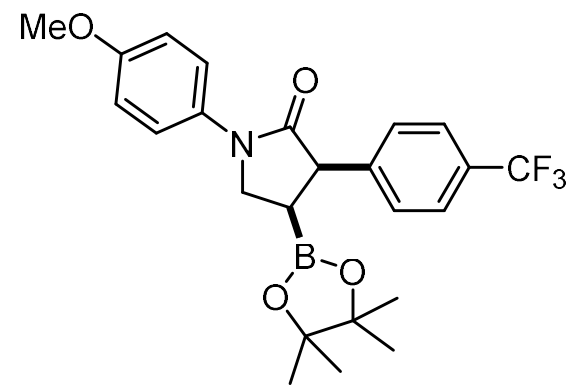

Prepared according to GP3. Isolated by flash column chromatography, $5 \% \rightarrow 25 \%$ EtOAc/Pentanes. Isolated $83 \mathrm{mg}$ of a white solid ( $90 \%$ yield).

${ }^{1} \mathbf{H}$ NMR $\left(500 \mathrm{MHz}, \mathrm{CDCl}_{3}\right) \delta 7.63(\mathrm{~d}, J=9.2 \mathrm{~Hz}, 2 \mathrm{H}), 7.58-$ $7.52(\mathrm{~m}, 2 \mathrm{H}), 7.39-7.33(\mathrm{~m}, 2 \mathrm{H}), 6.92(\mathrm{~d}, J=9.1 \mathrm{~Hz}, 2 \mathrm{H}), 4.20(\mathrm{t}$, $J=10.2 \mathrm{~Hz}, 1 \mathrm{H}), 4.04(\mathrm{~d}, J=10.2 \mathrm{~Hz}, 1 \mathrm{H}), 3.95$ (dd, $J=10.2,9.1$ $\mathrm{Hz}, 1 \mathrm{H}), 3.80(\mathrm{~s}, 3 \mathrm{H}), 2.46(\mathrm{td}, J=10.2,9.1 \mathrm{~Hz}, 1 \mathrm{H}), 0.95(\mathrm{~s}, 6 \mathrm{H})$, $0.93(\mathrm{~s}, 6 \mathrm{H})$.

${ }^{13} \mathbf{C}$ NMR $\left(126 \mathrm{MHz}, \mathrm{CDCl}_{3}\right) \delta 173.6,156.8,143.8(\mathrm{q}, J=1.3 \mathrm{~Hz})$, $132.4,129.6(\mathrm{q}, J=32.5 \mathrm{~Hz}), 125.5(\mathrm{q}, J=3.8 \mathrm{~Hz}), 124.1(\mathrm{q}, J=272.0 \mathrm{~Hz}), 121.8,114.1,83.9,55.5$, 51.5, 50.1, 24.5.

${ }^{19}$ F NMR $\left(377 \mathrm{MHz}, \mathrm{CDCl}_{3}\right) \delta-62.67$.

IR $\left(\right.$ ATR, $\left.\mathrm{cm}^{-1}\right) 1690,1517,1392,1325,1254,1124,1071,1026,838,678$

HRMS (DART) m/z: [M+H] ${ }^{+}$Calcd for $\mathrm{C}_{24} \mathrm{H}_{28} \mathrm{BNO}_{4} \mathrm{~F}_{3} 462.2058$; found 462.2067

MP $180-184{ }^{\circ} \mathrm{C}$ 
3-(4-fluorophenyl)-1-(4-methoxyphenyl)-4-(4,4,5,5-tetramethyl-1,3,2-dioxaborolan-2-yl)pyrrolidin2-one (2d)

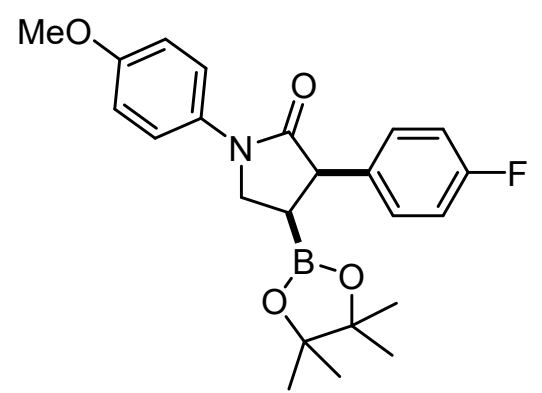

Prepared according to GP3. Isolated by flash column chromatography, $5 \%-\rightarrow 25 \%$ EtOAc/Pentanes. Isolated $80 \mathrm{mg}$ of a white solid ( $97 \%$ yield).

${ }^{1} \mathbf{H}$ NMR $\left(500 \mathrm{MHz}, \mathrm{CDCl}_{3}\right) \delta 7.63(\mathrm{~d}, J=9.2 \mathrm{~Hz}, 2 \mathrm{H}), 7.20(\mathrm{dd}, J=$ $8.8,5.2 \mathrm{~Hz}, 2 \mathrm{H}), 6.97$ (t, $J=8.8 \mathrm{~Hz}, 2 \mathrm{H}), 6.92(\mathrm{~d}, J=9.2 \mathrm{~Hz}, 2 \mathrm{H})$, 4.19 (t, $J=10.2 \mathrm{~Hz}, 1 \mathrm{H}), 3.95(\mathrm{~d}, J=10.2 \mathrm{~Hz}, 1 \mathrm{H}), 3.91$ (dd, $J=$ $10.2,9.1 \mathrm{~Hz}, 1 \mathrm{H}), 3.80$ (s, 3H), 2.41 (td, $J=10.2,9.1 \mathrm{~Hz}, 1 \mathrm{H}), 0.98$ (s, 6H), 0.97 (s, 6H).

${ }^{13}$ C NMR $\left(126 \mathrm{MHz}, \mathrm{CDCl}_{3}\right) \delta 174.3,162.1(\mathrm{~d}, J=245.4 \mathrm{~Hz}), 156.7$, $135.5(\mathrm{~d}, J=3.3 \mathrm{~Hz}), 132.6,130.1(\mathrm{~d}, J=8.1 \mathrm{~Hz}), 121.7,115.4(\mathrm{~d}, J=21.4 \mathrm{~Hz}), 114.1,83.8,55.5,51.0$, $50.1,24.7,24.6$.

${ }^{19}$ F NMR $\left(377 \mathrm{MHz}, \mathrm{CDCl}_{3}\right) \delta-115.51$.

IR $\left(\right.$ ATR, $\left.\mathrm{cm}^{-1}\right) 1690,1517,1387,1258,1217,1129,1022,857,825,692$

HRMS (DART) m/z: [M+H] $]^{+}$Calcd for $\mathrm{C}_{23} \mathrm{H}_{28} \mathrm{BNO}_{4} \mathrm{~F}$ 412.2090; found 412.2097

MP $149-150{ }^{\circ} \mathrm{C}$

3-(4-chlorophenyl)-1-(4-methoxyphenyl)-4-(4,4,5,5-tetramethyl-1,3,2-dioxaborolan-2-yl)pyrrolidin2-one (2e)

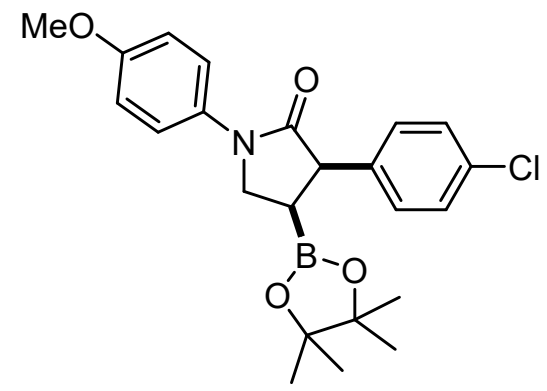

Prepared according to GP3. Isolated by flash column chromatography, $5 \% \rightarrow 25 \%$ EtOAc/Pentanes. Isolated $71 \mathrm{mg}$ of a white solid ( $83 \%$ yield).

${ }^{1} \mathbf{H}$ NMR $\left(500 \mathrm{MHz}, \mathrm{CDCl}_{3}\right) \delta 7.62(\mathrm{~d}, J=9.2 \mathrm{~Hz}, 2 \mathrm{H}), 7.25(\mathrm{~d}, J=$ $8.5 \mathrm{~Hz}, 2 \mathrm{H}), 7.17(\mathrm{~d}, J=8.5 \mathrm{~Hz}, 2 \mathrm{H}), 6.92(\mathrm{~d}, J=9.2 \mathrm{~Hz}, 2 \mathrm{H}), 4.18$ (t, $J=10.2 \mathrm{~Hz}, 1 \mathrm{H}), 3.94(\mathrm{~d}, J=10.2 \mathrm{~Hz}, 1 \mathrm{H}), 3.92(\mathrm{dd}, J=10.2,9.1$ $\mathrm{Hz}, 1 \mathrm{H}), 3.81$ (s, 3H), 2.42 (td, $J=10.2,9.1 \mathrm{~Hz}, 1 \mathrm{H}), 0.99$ (s, 6H), $0.97(\mathrm{~s}, 6 \mathrm{H})$

${ }^{13} \mathrm{C}$ NMR $\left(126 \mathrm{MHz}, \mathrm{CDCl}_{3}\right) \delta 174.0,156.7,138.2,133.1,132.6$, $129.9,128.7,121.8,114.1,83.9,55.5,51.1,50.2,24.6,24.6$.

IR $\left(\right.$ ATR, $\left.\mathrm{cm}^{-1}\right)$ 2983, 1690, 1516, 1387, 1325, 1249, 1133, 1030, 825, 696

HRMS (DART) m/z: [M+H] ${ }^{+}$Calcd for $\mathrm{C}_{23} \mathrm{H}_{28} \mathrm{BNO}_{4} \mathrm{Cl} 428.1794$; found 428.1793

MP $155-156{ }^{\circ} \mathrm{C}$

3-(4-bromophenyl)-1-(4-methoxyphenyl)-4-(4,4,5,5-tetramethyl-1,3,2-dioxaborolan-2-yl)pyrrolidin2-one (2f)

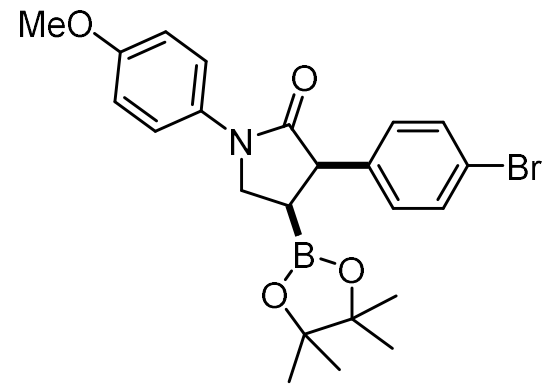

Prepared according to GP3. Isolated by flash column chromatography, $5 \% \rightarrow 25 \% \mathrm{EtOAc} /$ Pentanes. Isolated $55 \mathrm{mg}$ of a white solid (58\% yield).

${ }^{1} \mathbf{H}$ NMR $\left(500 \mathrm{MHz}, \mathrm{CDCl}_{3}\right) \delta 7.62(\mathrm{~d}, J=9.2 \mathrm{~Hz}, 2 \mathrm{H}), 7.41(\mathrm{~d}, J=$ $8.5 \mathrm{~Hz}, 2 \mathrm{H}), 7.11(\mathrm{~d}, J=8.5 \mathrm{~Hz}, 2 \mathrm{H}), 6.92(\mathrm{~d}, J=9.2 \mathrm{~Hz}, 2 \mathrm{H}), 4.18$ (t, $J=10.2 \mathrm{~Hz}, 1 \mathrm{H}), 3.96-3.88(\mathrm{~m}, 2 \mathrm{H}), 3.81(\mathrm{~s}, 3 \mathrm{H}), 2.42(\mathrm{td}, J=$ $10.2,9.0 \mathrm{~Hz}, 1 \mathrm{H}), 0.99(\mathrm{~s}, 6 \mathrm{H}), 0.98(\mathrm{~s}, 6 \mathrm{H})$.

${ }^{13} \mathrm{C}$ NMR $\left(126 \mathrm{MHz}, \mathrm{CDCl}_{3}\right) \delta 173.9,156.7,138.7,132.6,131.6$, $130.2,121.8,121.1,114.1,83.9,55.5,51.2,50.1,24.6,24.6$.

IR $\left(\right.$ ATR, $\left.\mathrm{cm}^{-1}\right)$ 2939, 1686, 1517, 1396, 1347, 1258, 1151, 1030, 824, 696

HRMS (DART) m/z: [M+H] ${ }^{+}$Calcd for $\mathrm{C}_{23} \mathrm{H}_{28} \mathrm{BNO}_{4} \mathrm{Br} 472.1289$; found 472.1289

MP $163-164{ }^{\circ} \mathrm{C}$ 
3-(3-methoxyphenyl)-1-(4-methoxyphenyl)-4-(4,4,5,5-tetramethyl-1,3,2-dioxaborolan-2yl)pyrrolidin-2-one (2g)

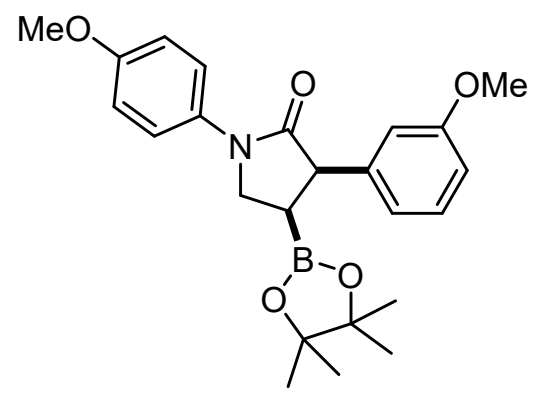

Prepared according to GP3. Isolated by flash column

chromatography, $5 \% \rightarrow 25 \%$ EtOAc/Pentanes. Isolated $79 \mathrm{mg}$ of a white solid ( $93 \%$ yield).

${ }^{1}$ H NMR $\left(500 \mathrm{MHz}, \mathrm{CDCl}_{3}\right) \delta 7.64(\mathrm{~d}, J=9.1 \mathrm{~Hz}, 1 \mathrm{H}), 7.17$ (ddd, $J$ $=8.1,7.4,0.6 \mathrm{~Hz}, 1 \mathrm{H}), 6.91(\mathrm{~d}, J=9.1 \mathrm{~Hz}, 2 \mathrm{H}), 6.83-6.77(\mathrm{~m}, 2 \mathrm{H})$, $6.75(\mathrm{ddd}, J=8.1,2.5,1.0 \mathrm{~Hz}, 1 \mathrm{H}), 4.23(\mathrm{t}, J=10.2 \mathrm{~Hz}, 1 \mathrm{H}), 3.93(\mathrm{~d}$, $J=10.2 \mathrm{~Hz}, 1 \mathrm{H}), 3.91$ (dd, $J=10.2,9.1 \mathrm{~Hz}, 1 \mathrm{H}), 3.80$ (s, 3H), 3.76 $(\mathrm{s}, 3 \mathrm{H}), 2.41(\mathrm{td}, J=10.2,9.1 \mathrm{~Hz}, 1 \mathrm{H}), 0.98(\mathrm{~s}, 6 \mathrm{H}), 0.96(\mathrm{~s}, 6 \mathrm{H})$. ${ }^{13}$ C NMR $\left(126 \mathrm{MHz}, \mathrm{CDCl}_{3}\right) \delta 174.5,159.7,156.6,141.0,132.8$, $129.7,121.8,120.4,114.3,114.0,112.9,83.7,55.5,55.2,51.8,50.3,24.6$. IR $\left(\right.$ ATR, $\left.\mathrm{cm}^{-1}\right) 2828,1690,1517,1387,1329,1240,1142,1040,830,785,696$ HRMS (DART) m/z: $[\mathrm{M}+\mathrm{H}]^{+}$Calcd for $\mathrm{C}_{24} \mathrm{H}_{30} \mathrm{BNO}_{5} 424.2290$; found 424.2293 MP $108-110{ }^{\circ} \mathrm{C}$

3-(3-fluorophenyl)-1-(4-methoxyphenyl)-4-(4,4,5,5-tetramethyl-1,3,2-dioxaborolan-2-yl)pyrrolidin2-one (2h)

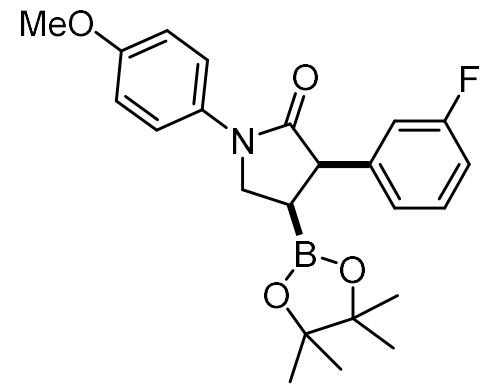

Prepared according to GP3. Isolated by flash column chromatography, $5 \% \rightarrow 25 \%$ EtOAc/Pentanes. Isolated $76 \mathrm{mg}$ of a white solid ( $92 \%$ yield). ${ }^{1} \mathbf{H}$ NMR $\left(500 \mathrm{MHz}, \mathrm{CDCl}_{3}\right) \delta 7.63(\mathrm{~d}, J=9.1 \mathrm{~Hz}, 2 \mathrm{H}), 7.24(\mathrm{td}, J=8.0$, $6.0 \mathrm{~Hz}, 1 \mathrm{H}), 7.01(\mathrm{ddd}, J=8.0,1.8,0.9 \mathrm{~Hz}, 1 \mathrm{H}), 6.96(\mathrm{ddd}, J=9.9,2.5$, $1.8 \mathrm{~Hz}, 1 \mathrm{H}), 6.94-6.89(\mathrm{~m}, 3 \mathrm{H}), 4.20(\mathrm{t}, J=10.2 \mathrm{~Hz}, 1 \mathrm{H}), 3.96(\mathrm{~d}, J=$ $10.2 \mathrm{~Hz}, 1 \mathrm{H}), 3.92(\mathrm{dd}, J=10.2,9.1 \mathrm{~Hz}, 1 \mathrm{H}), 3.81(\mathrm{~s}, 3 \mathrm{H}), 2.43(\mathrm{td}, J=$ $10.2,9.1 \mathrm{~Hz}, 1 \mathrm{H}), 0.99(\mathrm{~s}, 6 \mathrm{H}), 0.98(\mathrm{~s}, 6 \mathrm{H})$.

${ }^{13}$ C NMR $\left(126 \mathrm{MHz}, \mathrm{CDCl}_{3}\right) \delta 173.9,162.8(\mathrm{~d}, J=246.1 \mathrm{~Hz}), 156.7$, $142.0(\mathrm{~d}, J=7.2 \mathrm{~Hz}), 132.6,130.1(\mathrm{~d}, J=8.4 \mathrm{~Hz}), 124.1(\mathrm{~d}, J=2.9 \mathrm{~Hz})$, $121.8,115.6(\mathrm{~d}, J=21.7 \mathrm{~Hz}), 114.1(\mathrm{~d}, J=21.0 \mathrm{~Hz}), 114.1,83.8,55.5,51.5(\mathrm{~d}, J=1.8 \mathrm{~Hz}), 50.1,24.6$, 24.6 .

${ }^{19}$ F NMR $\left(377 \mathrm{MHz}, \mathrm{CDCl}_{3}\right) \delta-113.07$.

IR $\left(\right.$ ATR, $\left.\mathrm{cm}^{-1}\right) 2975,1682,1583,1516,1378,1334,1249,1151,830,785$

HRMS (DART) m/z: $[\mathrm{M}+\mathrm{H}]^{+}$Calcd for $\mathrm{C}_{23} \mathrm{H}_{28} \mathrm{BNO}_{4} \mathrm{~F} 412.2090$; found 412.2099

MP $124-126^{\circ} \mathrm{C}$

3-(3-chlorophenyl)-1-(4-methoxyphenyl)-4-(4,4,5,5-tetramethyl-1,3,2-dioxaborolan-2-yl)pyrrolidin2-one (2i)

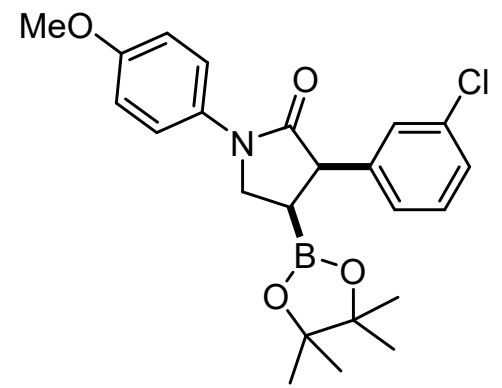

Prepared according to GP3. Isolated by flash column chromatography, $5 \% \rightarrow 25 \%$ EtOAc/Pentanes. Isolated $84 \mathrm{mg}$ of a white solid $(95 \%$ yield).

${ }^{1} \mathbf{H}$ NMR $\left(500 \mathrm{MHz}, \mathrm{CDCl}_{3}\right) \delta 7.63(\mathrm{~d}, J=9.1 \mathrm{~Hz}, 2 \mathrm{H}), 7.27-7.18(\mathrm{~m}$, $3 \mathrm{H}), 7.13-7.07(\mathrm{~m}, 1 \mathrm{H}), 6.92(\mathrm{~d}, J=9.1 \mathrm{~Hz}, 2 \mathrm{H}), 4.20(\mathrm{t}, J=10.2 \mathrm{~Hz}$, $1 \mathrm{H}), 3.97-3.88(\mathrm{~m}, 2 \mathrm{H}), 3.81(\mathrm{~s}, 3 \mathrm{H}), 2.43(\mathrm{td}, J=10.2,9.0 \mathrm{~Hz}, 1 \mathrm{H})$, $1.00(\mathrm{~s}, 6 \mathrm{H}), 0.98(\mathrm{~s}, 6 \mathrm{H})$.

${ }^{13} \mathbf{C}$ NMR $\left(126 \mathrm{MHz}, \mathrm{CDCl}_{3}\right) \delta 173.7,156.7,141.5,134.4,132.5$, $129.9,128.9,127.4,126.5,121.8,114.1,83.9,55.4,51.4,50.1,24.7$,

24.6.

IR $\left(\right.$ ATR, $\left.\mathrm{cm}^{-1}\right) 2975,1695,1508,1401,1240,1171,1035,830,794,683$ 
HRMS (DART) m/z: [M+H] $]^{+}$Calcd for $\mathrm{C}_{23} \mathrm{H}_{28} \mathrm{BNO}_{4} \mathrm{Cl}$ 428.1794; found 428.1797

MP $132-133{ }^{\circ} \mathrm{C}$

3-(3-bromophenyl)-1-(4-methoxyphenyl)-4-(4,4,5,5-tetramethyl-1,3,2-dioxaborolan-2-yl)pyrrolidin2-one (2j)

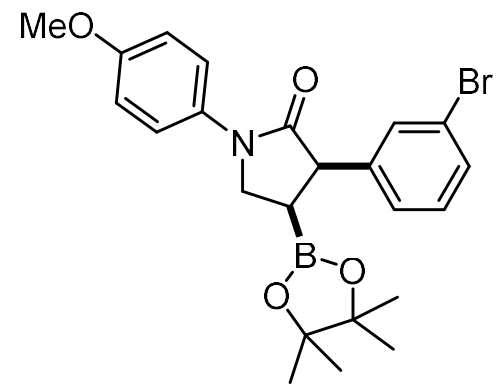

Prepared according to GP3. Isolated by flash column chromatography, $5 \% \rightarrow 25 \%$ EtOAc/Pentanes. Isolated $79 \mathrm{mg}$ of a white solid $(84 \%$ yield).

${ }^{1} \mathbf{H}$ NMR $\left(500 \mathrm{MHz}, \mathrm{CDCl}_{3}\right) \delta 7.63(\mathrm{~d}, J=9.1 \mathrm{~Hz}, 2 \mathrm{H}), 7.41-7.39(\mathrm{~m}$, $1 \mathrm{H}), 7.35$ (ddd, $J=5.7,3.7,2.0 \mathrm{~Hz}, 1 \mathrm{H}), 7.16-7.13$ (m, 2H), 6.92 (d, $J$ $=9.1 \mathrm{~Hz}, 2 \mathrm{H}), 4.19(\mathrm{t}, J=10.2 \mathrm{~Hz}, 1 \mathrm{H}), 3.93(\mathrm{~d}, J=10.2 \mathrm{~Hz}, 1 \mathrm{H}), 3.91$ (dd, $J=10.2,9.1 \mathrm{~Hz}, 1 \mathrm{H}), 3.80(\mathrm{~s}, 3 \mathrm{H}), 2.42(\mathrm{td}, J=10.2,9.1 \mathrm{~Hz}, 1 \mathrm{H})$, $1.00(\mathrm{~s}, 6 \mathrm{H}), 0.98(\mathrm{~s}, 6 \mathrm{H})$.

${ }^{13} \mathbf{C ~ N M R}\left(126 \mathrm{MHz}, \mathrm{CDCl}_{3}\right) \delta 173.6,156.7,141.8,132.5,131.8$,

$130.3,130.2,126.9,122.7,121.8,114.1,83.9,55.5,51.4,50.1,24.7,24.7$.

IR $\left(\mathrm{ATR}, \mathrm{cm}^{-1}\right)$ 2983, 1690, 1512, 1392, 1245, 1138, 1026, 821, 770, 687

HRMS (DART) m/z: [M+H $]^{+}$Calcd for $\mathrm{C}_{23} \mathrm{H}_{28} \mathrm{BNO}_{4} \mathrm{Br} 472.1289$; found 472.1282

MP $131-135^{\circ} \mathrm{C}$

3-(2-methoxyphenyl)-1-(4-methoxyphenyl)-4-(4,4,5,5-tetramethyl-1,3,2-dioxaborolan-2yl)pyrrolidin-2-one (2k)

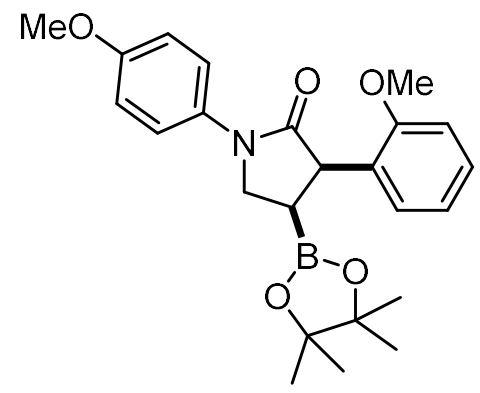

Prepared according to GP3. Isolated by flash column chromatography, $5 \% \rightarrow 25 \%$ EtOAc/Pentanes. Isolated $20 \mathrm{mg}$ of a white solid ( $23 \%$ yield).

${ }^{1}$ H NMR ${ }^{1} \mathrm{H}$ NMR $\left(500 \mathrm{MHz}, \mathrm{CDCl}_{3}\right) \delta 7.68-7.55(\mathrm{~m}, 2 \mathrm{H}), 7.22-7.09$ $(\mathrm{m}, 2 \mathrm{H}), 6.96-6.89(\mathrm{~m}, 2 \mathrm{H}), 6.86(\mathrm{td}, J=7.5,1.1 \mathrm{~Hz}, 1 \mathrm{H}), 6.82-6.73$ $(\mathrm{m}, 1 \mathrm{H}), 4.19(\mathrm{t}, J=9.3 \mathrm{~Hz}, 1 \mathrm{H}), 3.87(\mathrm{t}, J=9.3 \mathrm{~Hz}, 1 \mathrm{H}), 3.81(\mathrm{~s}, 3 \mathrm{H})$, $3.76(\mathrm{~s}, 3 \mathrm{H}), 2.42(\mathrm{dt}, J=11.3,9.3 \mathrm{~Hz}, 1 \mathrm{H}), 1.31-1.19(\mathrm{~m}, 1 \mathrm{H}), 0.95(\mathrm{~s}$, $6 \mathrm{H}), 0.89(\mathrm{~s}, 6 \mathrm{H})$.

${ }^{13}$ C NMR $\left(126 \mathrm{MHz}, \mathrm{CDCl}_{3}\right) \delta 174.9,157.3,156.5,133.2,128.4,121.9$, 120.6, 114.0, 110.6, 83.4, 55.4, 55.5, 50.7, 24.7, 24.7, 24.6, 1.01.

IR $\left(\mathrm{ATR}, \mathrm{cm}^{-1}\right)$ 2946, 1690, 1530, 1387, 1329, 1249, 1035, 834, 776,

678

HRMS (DART) m/z: $[\mathrm{M}+\mathrm{H}]^{+}$Calcd for $\mathrm{C}_{24} \mathrm{H}_{31} \mathrm{BNO}_{5} 424.2290$; found 424.2293

MP $220-222{ }^{\circ} \mathrm{C}$

3-(furan-2-yl)-1-(4-methoxyphenyl)-4-(4,4,5,5-tetramethyl-1,3,2-dioxaborolan-2-yl)pyrrolidin-2-one (2l)

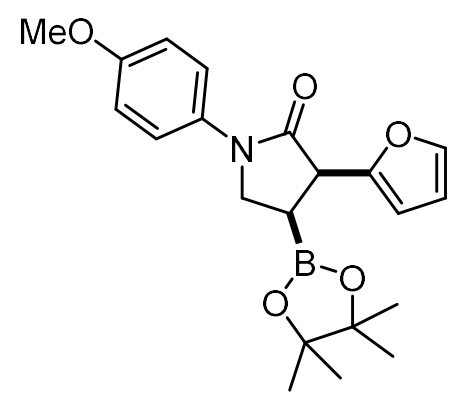

Prepared according to GP3. Isolated by flash column chromatography, $5 \% \rightarrow 25 \%$ EtOAc/Pentanes. Isolated $72 \mathrm{mg}$ of a white solid ( $94 \%$ yield). ${ }^{1} \mathbf{H}$ NMR $\left(500 \mathrm{MHz}, \mathrm{CDCl}_{3}\right) \delta 7.58(\mathrm{~d}, J=9.1 \mathrm{~Hz}, 2 \mathrm{H}), 7.28(\mathrm{dd}, J=1.9$, $0.9 \mathrm{~Hz}, 1 \mathrm{H}), 6.90(\mathrm{~d}, J=9.1 \mathrm{~Hz}, 2 \mathrm{H}), 6.28(\mathrm{dd}, J=3.2,1.9 \mathrm{~Hz}, 1 \mathrm{H}), 6.27-$ $6.26(\mathrm{~m}, 1 \mathrm{H}), 4.26(\mathrm{dd}, J=10.8,9.6 \mathrm{~Hz}, 1 \mathrm{H}), 4.04(\mathrm{~d}, J=9.6 \mathrm{~Hz}, 1 \mathrm{H})$, $3.86(\mathrm{dd}, J=9.6,8.6 \mathrm{~Hz}, 1 \mathrm{H}), 3.79(\mathrm{~s}, 3 \mathrm{H}), 2.32(\mathrm{ddd}, J=10.8,9.6,8.6$ $\mathrm{Hz}, 1 \mathrm{H}), 1.14(\mathrm{~s}, 6 \mathrm{H}), 1.09(\mathrm{~s}, 6 \mathrm{H})$.

${ }^{13}$ C NMR $\left(126 \mathrm{MHz}, \mathrm{CDCl}_{3}\right) \delta 171.9,156.6,152.4,141.5,132.8,121.8$, $114.0,110.5,107.7,83.8,55.5,50.4,45.6,24.9,24.6$.

IR $\left(\right.$ ATR, $\left.\mathrm{cm}^{-1}\right) 2979,1686,1512,1383,1254,1147,1048,830,750,696$

HRMS (DART) m/z: $[\mathrm{M}+\mathrm{H}]^{+}$Calcd for $\mathrm{C}_{21} \mathrm{H}_{27} \mathrm{BNO}_{5} 384.1977$; found 384.1972 
MP $127-128^{\circ} \mathrm{C}$

1-(4-methoxyphenyl)-4-(4,4,5,5-tetramethyl-1,3,2-dioxaborolan-2-yl)-3-(thiophen-2-yl)pyrrolidin-2one (2m)

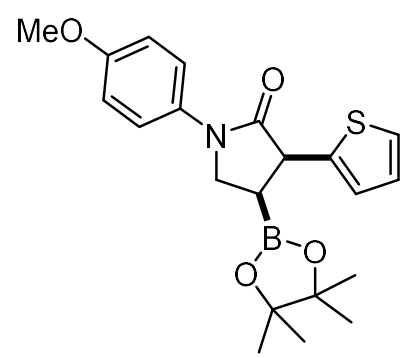

Prepared according to GP3. Isolated by flash column chromatography, $5 \% \rightarrow 30 \%$ EtOAc/Pentanes. Isolated $70 \mathrm{mg}$ of a white solid ( $88 \%$ yield).

${ }^{1} \mathbf{H}$ NMR $\left(500 \mathrm{MHz}, \mathrm{CDCl}_{3}\right) \delta 7.64-7.53(\mathrm{~m}, 2 \mathrm{H}), 7.14(\mathrm{dd}, J=5.1,1.2 \mathrm{~Hz}$, $1 \mathrm{H}), 6.97(\mathrm{ddd}, J=3.5,1.2,0.5 \mathrm{~Hz}, 1 \mathrm{H}), 6.93-6.87(\mathrm{~m}, 3 \mathrm{H}), 4.31-4.16(\mathrm{~m}$, $2 \mathrm{H}), 3.92(\mathrm{dd}, J=10.1,8.6 \mathrm{~Hz}, 1 \mathrm{H}), 2.40(\mathrm{dt}, J=10.5,8.6 \mathrm{~Hz}, 1 \mathrm{H}), 1.05(\mathrm{~s}$, $6 \mathrm{H}), 1.02(\mathrm{~s}, 6 \mathrm{H})$.

${ }^{13}$ C NMR $\left(126 \mathrm{MHz}, \mathrm{CDCl}_{3}\right) \delta 173.3,156.7,140.9,132.7,126.7,126.0$, $124.2,122.0,114.1,83.8,55.5,50.0,47.0,24.7,24.6$.

IR $\left(\right.$ ATR, $\left.\mathrm{cm}^{-1}\right) 1689,1521,1386,1327,1236,1146,1033,988,834,725$

MP $126-128^{\circ} \mathrm{C}$

\section{1,3-diphenyl-4-(4,4,5,5-tetramethyl-1,3,2-dioxaborolan-2-yl)pyrrolidin-2-one (2n)}

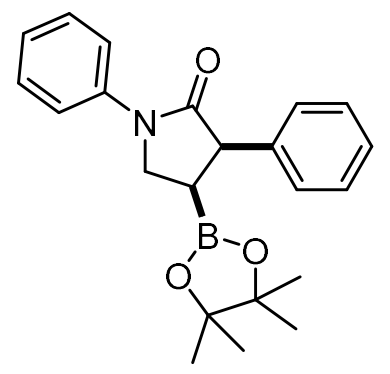

Prepared according to GP3. Isolated by flash column chromatography, $5 \% \rightarrow 25 \%$ EtOAc/Pentanes. Isolated $62 \mathrm{mg}$ of a white solid ( $85 \%$ yield).

${ }^{1} \mathbf{H}$ NMR $\left(500 \mathrm{MHz}, \mathrm{CDCl}_{3}\right) \delta 7.79-7.74(\mathrm{~m}, 2 \mathrm{H}), 7.42-7.37(\mathrm{~m}, 2 \mathrm{H})$, $7.29-7.26(\mathrm{~m}, 1 \mathrm{H}), 7.26-7.18(\mathrm{~m}, 4 \mathrm{H}), 7.18-7.14(\mathrm{~m}, 1 \mathrm{H}), 4.28(\mathrm{dd}, J=$ $10.7,10.0 \mathrm{~Hz}, 1 \mathrm{H}), 3.99(\mathrm{~d}, J=10.0 \mathrm{~Hz}, 1 \mathrm{H}), 3.96(\mathrm{dd}, J=10.0,9.0 \mathrm{~Hz}, 1 \mathrm{H})$, $2.48-2.37(\mathrm{~m}, 1 \mathrm{H}), 0.96(\mathrm{~s}, 6 \mathrm{H}), 0.95(\mathrm{~s}, 6 \mathrm{H})$.

${ }^{13} \mathrm{C}$ NMR $\left(126 \mathrm{MHz}, \mathrm{CDCl}_{3}\right) \delta 175.0,139.5,139.5,128.8,128.6,128.4,127.3$, 124.5, 120.0, 83.8, 52.0, 49.9, 24.7, 24.6.

IR $\left(\mathrm{ATR}, \mathrm{cm}^{-1}\right) 2983,1699,1606,1512,1383,1155,990,852,763,691$

HRMS (DART) m/z: $[\mathrm{M}+\mathrm{H}]^{+}$Calcd for $\mathrm{C}_{22} \mathrm{H}_{27} \mathrm{BNO}_{3} 364.2079$; found 364.2075

MP $126-128^{\circ} \mathrm{C}$

3-phenyl-4-(4,4,5,5-tetramethyl-1,3,2-dioxaborolan-2-yl)-1-(p-tolyl)pyrrolidin-2-one (2o)

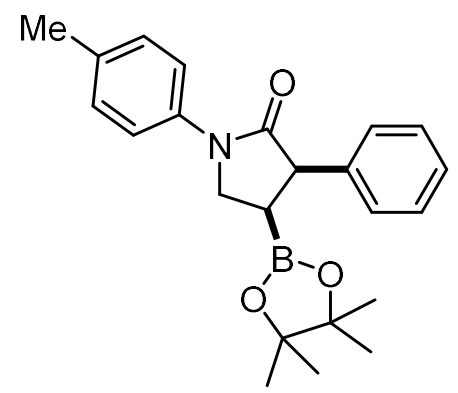

Prepared according to GP3. Isolated by flash column chromatography, $5 \% \rightarrow 25 \%$ EtOAc/Pentanes. Isolated $57 \mathrm{mg}$ of a white solid (76\% yield). ${ }^{1} \mathbf{H}$ NMR $\left(500 \mathrm{MHz}, \mathrm{CDCl}_{3}\right) \delta 7.64(\mathrm{~d}, J=8.6 \mathrm{~Hz}, 2 \mathrm{H}), 7.28-7.24(\mathrm{~m}$, $2 \mathrm{H}), 7.23-7.17(\mathrm{~m}, 4 \mathrm{H}), 4.25(\mathrm{t}, J=10.3 \mathrm{~Hz}, 1 \mathrm{H}), 3.97(\mathrm{~d}, J=10.3 \mathrm{~Hz}$, $1 \mathrm{H}), 3.93(\mathrm{dd}, J=10.3,9.1 \mathrm{~Hz}, 1 \mathrm{H}), 2.42(\mathrm{td}, J=10.3,9.1 \mathrm{~Hz}, 1 \mathrm{H}), 2.34$ $(\mathrm{s}, 3 \mathrm{H}), 0.95(\mathrm{~s}, 12 \mathrm{H})$.

${ }^{13}$ C NMR $\left(126 \mathrm{MHz}, \mathrm{CDCl}_{3}\right) \delta 174.8,139.6,137.0,134.2,129.4,128.6$, $128.4,127.2,120.0,83.7,52.0,49.9,24.7,24.6,20.9$.

IR $\left(\mathrm{ATR}, \mathrm{cm}^{-1}\right)$ 2989, 1690, 1517, 1490, 1392, 1147, 990, 861, 812, 723

HRMS (DART) m/z: [M+H] $]^{+}$Calcd for $\mathrm{C}_{23} \mathrm{H}_{29} \mathrm{BNO}_{3} 378.2235$; found 378.2234

MP $169-170{ }^{\circ} \mathrm{C}$

1-(4-methoxybenzyl)-3-phenyl-4-(4,4,5,5-tetramethyl-1,3,2-dioxaborolan-2-yl)pyrrolidin-2-one (2p) 
<smiles>COc1ccc(CN2CC(B3OC(C)(C)C(C)(C)O3)[C@H](c3ccccc3)C2=O)cc1</smiles>

Prepared according to GP3. Isolated by flash column chromatography, $5 \% \rightarrow 40 \%$ EtOAc/Pentanes. Isolated $71 \mathrm{mg}$ of a white solid ( $87 \%$ yield).

${ }^{1} \mathbf{H}$ NMR $\left(500 \mathrm{MHz}, \mathrm{CDCl}_{3}\right) \delta 7.25-7.16(\mathrm{~m}, 5 \mathrm{H}), 7.15-7.09(\mathrm{~m}$, $2 \mathrm{H}), 6.90-6.85(\mathrm{~m}, 2 \mathrm{H}), 4.56(\mathrm{~d}, J=14.4 \mathrm{~Hz}, 1 \mathrm{H}), 4.42(\mathrm{~d}, J=14.4$ $\mathrm{Hz}, 1 \mathrm{H}), 3.87-3.75(\mathrm{~m}, 4 \mathrm{H}), 3.59(\mathrm{t}, J=10.1 \mathrm{~Hz}, 1 \mathrm{H}), 3.35(\mathrm{dd}, J=$ $10.1,9.1 \mathrm{~Hz}, 1 \mathrm{H}), 2.24(\mathrm{td}, J=10.1,9.1 \mathrm{~Hz}, 1 \mathrm{H}), 0.89(\mathrm{~s}, 6 \mathrm{H}), 0.85$ $(\mathrm{s}, 6 \mathrm{H})$.

${ }^{13}$ C NMR $\left(126 \mathrm{MHz}, \mathrm{CDCl}_{3}\right)$ 175.6, 159.1, 140.0, 129.8, 128.8, 128.5, 128.3, 127.1, 114.0, 83.5, 55.3, 50.5, 47.7, 46.4, 24.6, 24.5.

IR $\left(\right.$ ATR, $\left.\mathrm{cm}^{-1}\right) 1697,1496,1382,1332,1240,1144,1035,870,769,692$

HRMS (DART) m/z: $[\mathrm{M}+\mathrm{H}]^{+}$Calcd for $\mathrm{C}_{24} \mathrm{H}_{30} \mathrm{BNO}_{4} 408.2341$; found 408.2352 MP $95-97{ }^{\circ} \mathrm{C}$

(3,4,5)-1-(4-methoxyphenyl)-5-methyl-3-phenyl-4-(4,4,5,5-tetramethyl-1,3,2-dioxaborolan-2yl)pyrrolidin-2-one (2q)<smiles>COc1ccc(N2C(=O)C(c3ccccc3)C(B3OC(C)(C)C(C)(C)O3)C2C)cc1</smiles>

Prepared according to GP3. Isolated by flash column chromatography, $5 \% \rightarrow 30 \%$ EtOAc/Pentanes. Isolated $56 \mathrm{mg}$ of a white solid (69\% yield). ${ }^{1} \mathbf{H}$ NMR $\left(500 \mathrm{MHz}, \mathrm{CDCl}_{3}\right) \delta 7.55-7.47(\mathrm{~m}, 2 \mathrm{H}), 7.32-7.23(\mathrm{~m}, 4 \mathrm{H})$, $7.22-7.13(\mathrm{~m}, 1 \mathrm{H}), 6.98-6.91(\mathrm{~m}, 2 \mathrm{H}), 4.32(\mathrm{p}, J=6.6 \mathrm{~Hz}, 1 \mathrm{H}), 4.10$ $(\mathrm{d}, J=9.5 \mathrm{~Hz}, 1 \mathrm{H}), 2.56(\mathrm{dd}, J=9.5,6.6 \mathrm{~Hz}, 1 \mathrm{H}), 1.21(\mathrm{~d}, J=6.6 \mathrm{~Hz}$, $3 \mathrm{H}), 1.01(\mathrm{~s}, 6 \mathrm{H}), 0.92(\mathrm{~s}, 6 \mathrm{H})$.

${ }^{13}$ C NMR $\left(126 \mathrm{MHz}, \mathrm{CDCl}_{3}\right) \delta 174.1,157.9,138.3,130.5,129.5,128.0$, $127.1,126.7,114.3,83.5,55.5,55.4,49.7,25.1,24.5,18.2$.

IR $\left(\right.$ ATR, $\left.\mathrm{cm}^{-1}\right)$ 2983, 1690, 1512, 1382, 1240, 1146, 1035, 977, 847, 709

MP $154-155^{\circ} \mathrm{C}$

(R)-1-(4-methoxyphenyl)-3-phenyl-3-((4,4,5,5-tetramethyl-1,3,2-dioxaborolan-2yl)methyl)pyrrolidin-2-one (4a)<smiles>COc1ccc(N2CC[C@](CB3OC(C)(C)C(C)(C)O3)(c3ccccc3)C2=O)cc1</smiles>

Prepared according to GP4. Isolated by flash column chromatography, $5 \% \rightarrow 30 \%$ EtOAc/Pentanes. Isolated $78 \mathrm{mg}$ of a white solid (95\% yield, 97:3 er).

${ }^{1} \mathbf{H}$ NMR $\left(500 \mathrm{MHz}, \mathrm{CDCl}_{3}\right) \delta 7.59-7.53(\mathrm{~m}, 2 \mathrm{H}), 7.49(\mathrm{dd}, J=7.4,1.3$ $\mathrm{Hz}, 2 \mathrm{H}), 7.35-7.27(\mathrm{~m}, 2 \mathrm{H}), 7.23-7.17(\mathrm{~m}, 1 \mathrm{H}), 6.93-6.84(\mathrm{~m}, 2 \mathrm{H})$, $3.79(\mathrm{~s}, 3 \mathrm{H}), 3.73-3.68(\mathrm{~m}, 2 \mathrm{H}), 2.71(\mathrm{ddd}, J=12.9,5.2,4.1 \mathrm{~Hz}, 1 \mathrm{H})$, $2.51(\mathrm{dt}, J=12.9,8.7 \mathrm{~Hz}, 1 \mathrm{H}), 1.73(\mathrm{~d}, J=15.8 \mathrm{~Hz}, 1 \mathrm{H}), 1.45(\mathrm{~d}, J=15.8$ $\mathrm{Hz}, 1 \mathrm{H}), 1.13(\mathrm{~s}, 6 \mathrm{H}), 1.13(\mathrm{~s}, 6 \mathrm{H})$.

${ }^{13} \mathbf{C}$ NMR $\left(126 \mathrm{MHz}, \mathrm{CDCl}_{3}\right) \delta 176.4,156.3,143.3,133.3,128.3,126.6$, 126.3, 121.5, 113.9, 83.1, 55.5, 50.9, 45.8, 32.8, 24.8, 24.7. IR $\left(\mathrm{ATR}, \mathrm{cm}^{-1}\right)$ 2992, 1695, 1516, 1369, 1329, 1249, 1151, 1035, 977, 825

HRMS (DART) m/z: $[\mathrm{M}+\mathrm{H}]^{+}$Calcd for $\mathrm{C}_{24} \mathrm{H}_{31} \mathrm{BNO}_{4} 408.2341$; found 408.2348 MP $124-129{ }^{\circ} \mathrm{C}$ $[\alpha]^{20}{ }_{\mathrm{D}}=+62.83\left(c=0.140, \mathrm{CH}_{2} \mathrm{Cl}_{2}\right)$

HPLC: OD, $10 \% \mathrm{IPA} /$ hexanes, $1 \mathrm{~mL} / \mathrm{min}$ 

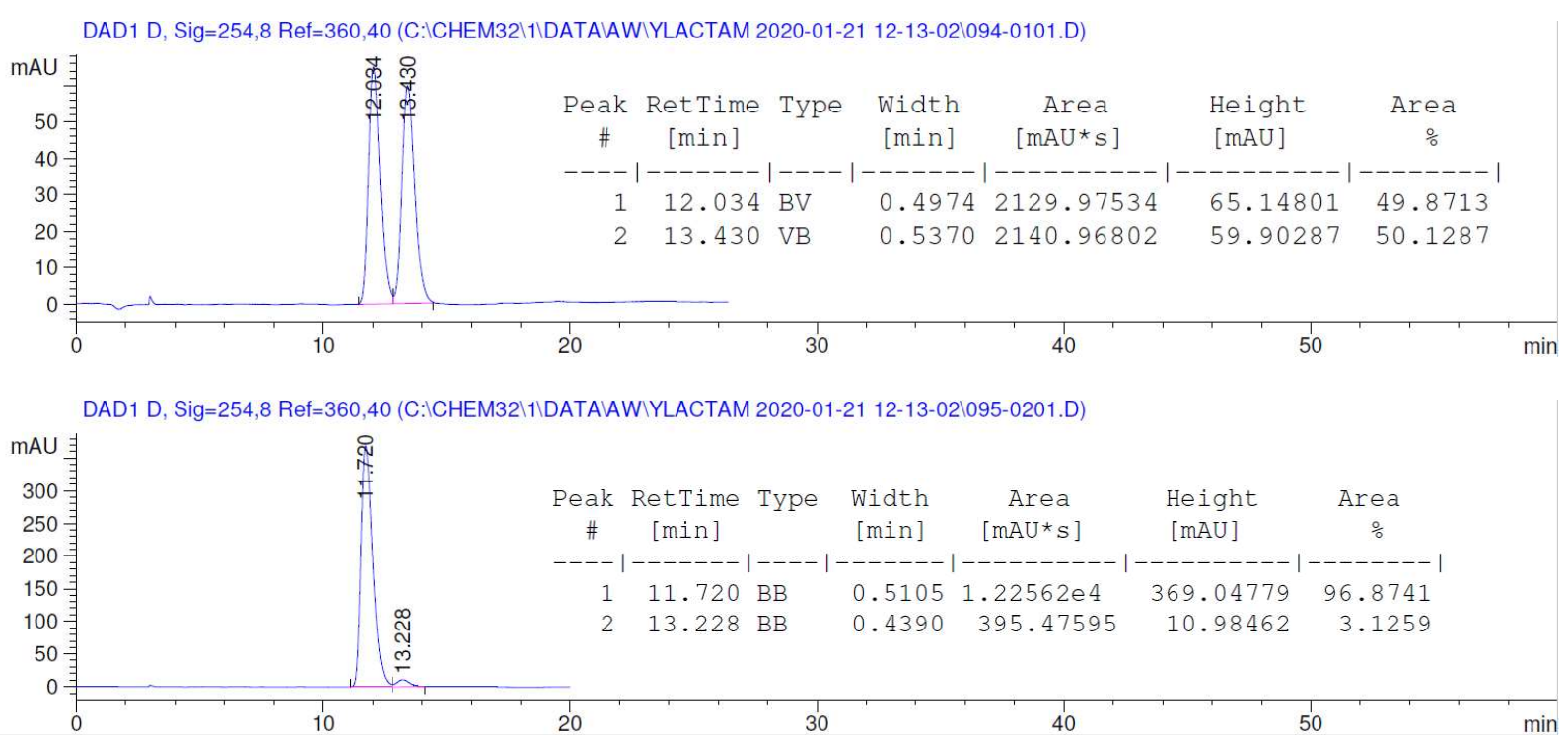

(R)-3-(2-methoxyphenyl)-1-(4-methoxyphenyl)-3-((4,4,5,5-tetramethyl-1,3,2-dioxaborolan-2yl)methyl)pyrrolidin-2-one (4b)<smiles></smiles>

Prepared according to GP4. Isolated by flash column chromatography, $5 \% \rightarrow 30 \%$ EtOAc/Pentanes. Isolated $56 \mathrm{mg}$ of a white solid (64\% yield, 89.5:10.5 er).

${ }^{1}$ H NMR $\left(500 \mathrm{MHz}, \mathrm{CDCl}_{3}\right) \delta 7.63-7.58(\mathrm{~m}, 2 \mathrm{H}), 7.29(\mathrm{dd}, J=7.6,1.6$ $\mathrm{Hz}, 1 \mathrm{H}), 7.21(\mathrm{ddd}, J=8.2,7.4,1.7 \mathrm{~Hz}, 1 \mathrm{H}), 6.93-6.84(\mathrm{~m}, 4 \mathrm{H}), 3.81$ $(\mathrm{s}, 3 \mathrm{H}), 3.80(\mathrm{~s}, 3 \mathrm{H}), 3.79-3.74(\mathrm{~m}, 1 \mathrm{H}), 3.71(\mathrm{td}, J=8.8,6.5 \mathrm{~Hz}, 1 \mathrm{H})$, 2.56 (ddd, $J=13.0,8.8,4.6 \mathrm{~Hz}, 1 \mathrm{H}), 2.44$ (ddd, $J=13.0,8.8,6.5 \mathrm{~Hz}$, $1 \mathrm{H}), 1.81(\mathrm{~d}, J=15.7 \mathrm{~Hz}, 1 \mathrm{H}), 1.52(\mathrm{~d}, J=15.7 \mathrm{~Hz}, 1 \mathrm{H}), 1.20(\mathrm{~s}, 6 \mathrm{H})$, $1.20(\mathrm{~s}, 6 \mathrm{H})$.

${ }^{13}$ C NMR (126 MHz, $\left.\mathrm{CDCl}_{3}\right) \delta 177.4,157.3,156.1,133.7,133.3,127.9,127.7,121.6,120.3,113.9$, $111.5,83.1,55.5,55.5,50.4,46.5,32.0,24.8$.

IR $\left(\right.$ ATR, $\left.\mathrm{cm}^{-1}\right)$ 2979, 1682, 1517, 1374, 1249, 1151, 1035, 973, 839, 754

HRMS (DART) m/z: [M+H] $]^{+}$Calcd for $\mathrm{C}_{25} \mathrm{H}_{33} \mathrm{BNO}_{5} 438.2447$; found 438.2453

MP $146-147^{\circ} \mathrm{C}$

$[\alpha]^{20}{ }_{\mathrm{D}}=+24.99\left(c=0.120, \mathrm{CH}_{2} \mathrm{Cl}_{2}\right)$

HPLC: OD, 5\% IPA/hexanes, $1 \mathrm{~mL} / \mathrm{min}$

DAD1 D, Sig=254,8 Ref=360,40 (C:ICHEM3211।DATAIPDIAW 2020-07-15 17-25-34l001-0101.D)

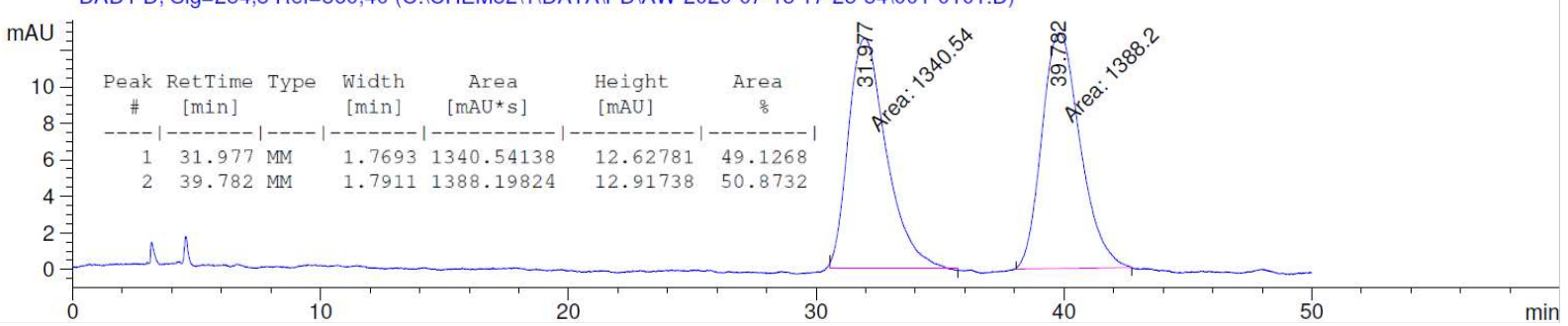




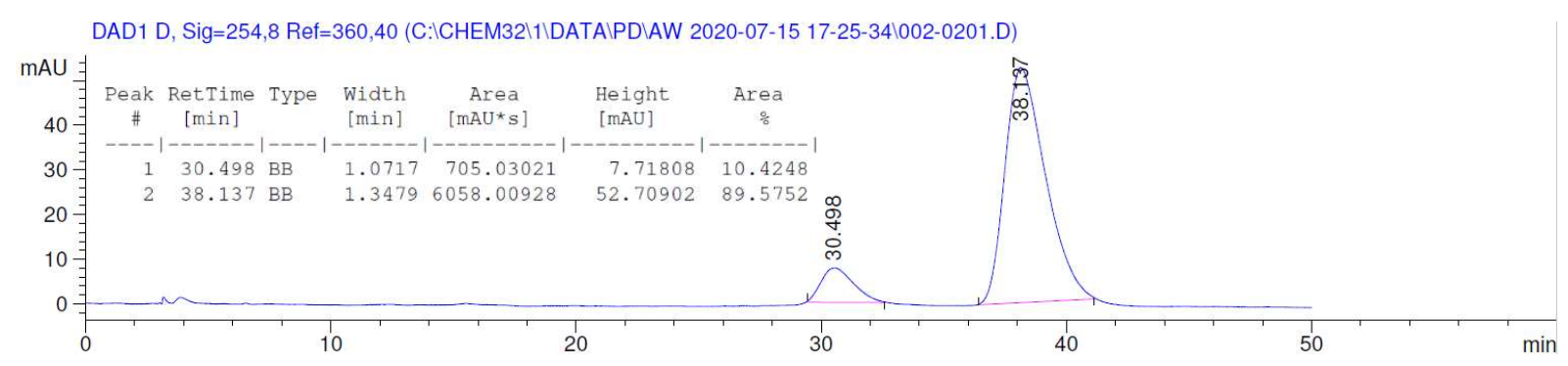

(R)-1-(4-methoxyphenyl)-3-((4,4,5,5-tetramethyl-1,3,2-dioxaborolan-2-yl)methyl)-3-( $m$ tolyl)pyrrolidin-2-one (4c)<smiles>COc1ccc(N2CC[C@](CB3OC(C)(C)C(C)(C)O3)(c3cccc(C)c3)C2=O)cc1</smiles>

Prepared according to GP4. Isolated by flash column chromatography, $5 \% \rightarrow 30 \%$ EtOAc/Pentanes. Isolated $72 \mathrm{mg}$ of a white solid ( $85 \%$ yield, $98: 2$ er).

${ }^{1}$ H NMR $\delta 7.59-7.48(\mathrm{~m}, 2 \mathrm{H}), 7.35-7.21(\mathrm{~m}, 2 \mathrm{H}), 7.18(\mathrm{t}, J=7.6$

$\mathrm{Hz}, 1 \mathrm{H}), 7.01$ (ddt, $J=7.6,1.9,0.9 \mathrm{~Hz}, 1 \mathrm{H}), 6.94-6.82(\mathrm{~m}, 2 \mathrm{H})$, $3.79(\mathrm{~s}, 3 \mathrm{H}), 3.76-3.65(\mathrm{~m}, 2 \mathrm{H}), 2.70$ (ddd, $J=12.9,5.6,3.6 \mathrm{~Hz}$, $1 \mathrm{H}), 2.50(\mathrm{dt}, J=12.9,8.7 \mathrm{~Hz}, 1 \mathrm{H}), 2.32(\mathrm{~d}, J=0.7 \mathrm{~Hz}, 3 \mathrm{H}), 1.70(\mathrm{~d}$, $J=15.8 \mathrm{~Hz}, 1 \mathrm{H}), 1.44(\mathrm{~d}, J=15.8 \mathrm{~Hz}, 1 \mathrm{H}), 1.14(\mathrm{~s}, 6 \mathrm{H}), 1.14$ (s, $6 \mathrm{H})$.

${ }^{13} \mathbf{C}$ NMR $\left(126 \mathrm{MHz}, \mathrm{CDCl}_{3}\right) \delta 176.5,156.3,143.2,137.8,133.4,128.2,127.4,127.2,123.3,121.5$, $113.9,83.1,55.5,50.9,45.8,32.9,24.8,24.7,21.6$.

IR $\left(\right.$ ATR, $\left.\mathrm{cm}^{-1}\right)$ 2979, 1690, 1516, 1400, 1360, 1258, 1142, 1039, 973, 834

HRMS (DART) m/z: [M+H] $]^{+}$Calcd for $\mathrm{C}_{25} \mathrm{H}_{33} \mathrm{BNO}_{4} 422.2497$; found 422.2498

MP $102-103{ }^{\circ} \mathrm{C}$

$[\alpha]^{20}{ }_{\mathrm{D}}=+64.26\left(c=0.280, \mathrm{CH}_{2} \mathrm{Cl}_{2}\right)$

HPLC: IA, 10\% IPA/hexanes, $1 \mathrm{~mL} / \mathrm{min}$

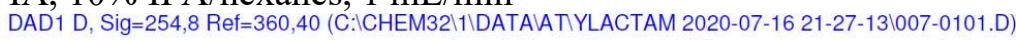

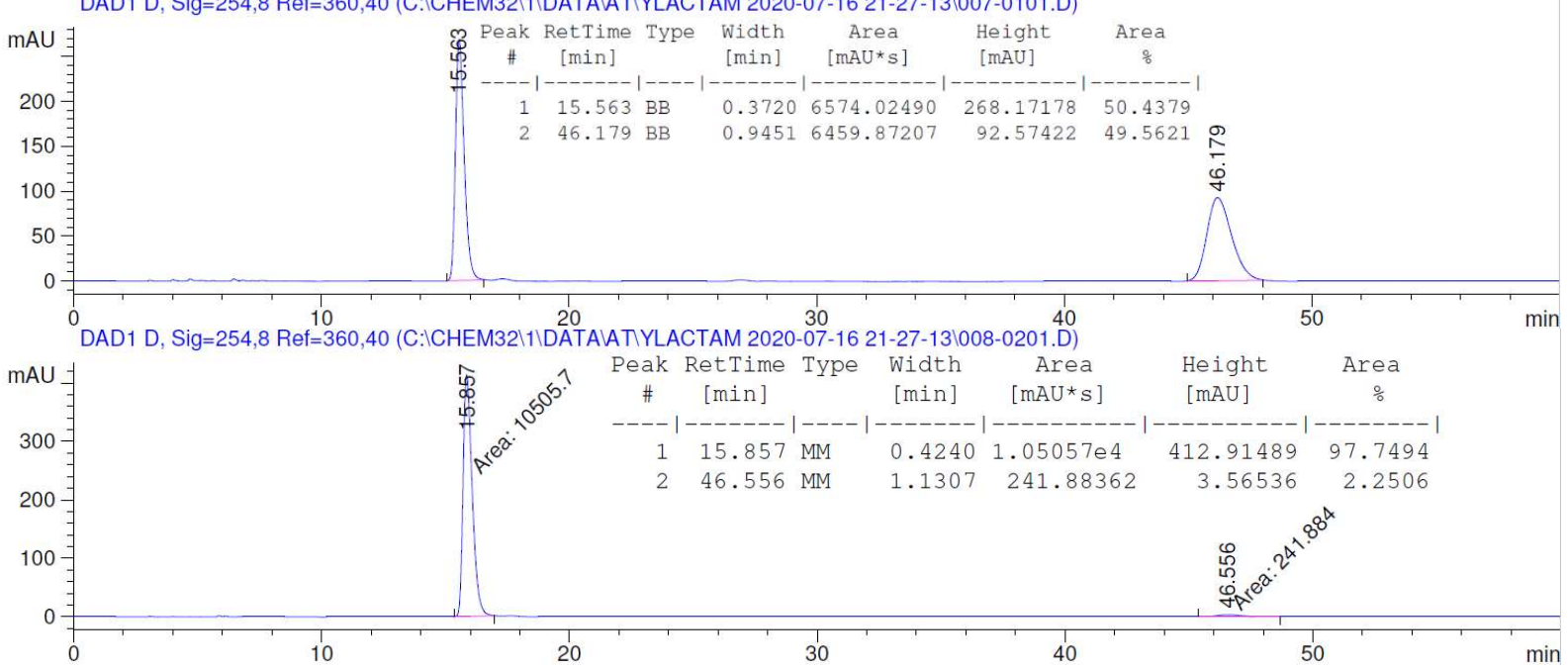

(R)-1-(4-methoxyphenyl)-3-(naphthalen-2-yl)-3-((4,4,5,5-tetramethyl-1,3,2-dioxaborolan-2yl)methyl)pyrrolidin-2-one (4d) 
<smiles>COc1ccc(N2CC[C@@](CB3OC(C)(C)C(C)(C)O3)(c3ccc4ccccc4c3)C2=O)cc1</smiles>

Prepared according to GP4. Isolated by flash column chromatography, $5 \% \rightarrow 30 \%$ EtOAc/Pentanes. Isolated $68 \mathrm{mg}$ of a white solid (74\% yield, 96.5:3.5 er).

${ }^{1} \mathbf{H}$ NMR $\left(500 \mathrm{MHz}, \mathrm{CDCl}_{3}\right) \delta 7.87(\mathrm{dd}, J=1.9,0.9 \mathrm{~Hz}, 1 \mathrm{H}), 7.82-$ $7.75(\mathrm{~m}, 3 \mathrm{H}), 7.68(\mathrm{dd}, J=8.7,2.0 \mathrm{~Hz}, 1 \mathrm{H}), 7.60-7.52(\mathrm{~m}, 2 \mathrm{H})$, $7.48-7.39(\mathrm{~m}, 2 \mathrm{H}), 6.92-6.86(\mathrm{~m}, 2 \mathrm{H}), 3.79(\mathrm{~s}, 3 \mathrm{H}), 3.74(\mathrm{dd}, J=$ 8.7, $4.7 \mathrm{~Hz}, 2 \mathrm{H}), 2.82(\mathrm{dt}, J=12.9,4.7 \mathrm{~Hz}, 1 \mathrm{H}), 2.61(\mathrm{dt}, J=12.9$, $8.7 \mathrm{~Hz}, 1 \mathrm{H}), 1.80(\mathrm{~d}, J=15.9 \mathrm{~Hz}, 1 \mathrm{H}), 1.55(\mathrm{~d}, J=15.9 \mathrm{~Hz}, 1 \mathrm{H})$, $1.13(\mathrm{~s}, 6 \mathrm{H}), 1.12(\mathrm{~s}, 6 \mathrm{H})$.

${ }^{13}$ C NMR $\left(126 \mathrm{MHz}, \mathrm{CDCl}_{3}\right) \delta 176.3,156.4,140.7,133.3,133.1,132.3,128.2,128.1,127.4,125.9$, $125.7,125.1,124.6,121.6,114.0,83.2,55.5,51.3,45.9,32.9,24.8,24.7$.

IR $\left(\right.$ ATR, $\left.\mathrm{cm}^{-1}\right)$ 2983, 1680, 1512, 1363, 1326, 1258, 1149, 1031, 972, 831, 754

HRMS (DART) $\mathrm{m} / \mathrm{z}$ : $[\mathrm{M}+\mathrm{H}]^{+}$Calcd for $\mathrm{C}_{28} \mathrm{H}_{33} \mathrm{BNO}_{4} 458.2497$; found 458.2501

MP $115-116^{\circ} \mathrm{C}$ $[\alpha]^{20}{ }_{\mathrm{D}}=+86.91\left(c=0.115, \mathrm{CH}_{2} \mathrm{Cl}_{2}\right)$

HPLC: OD, 20\% IPA/hexanes, $1 \mathrm{~mL} / \mathrm{min}$
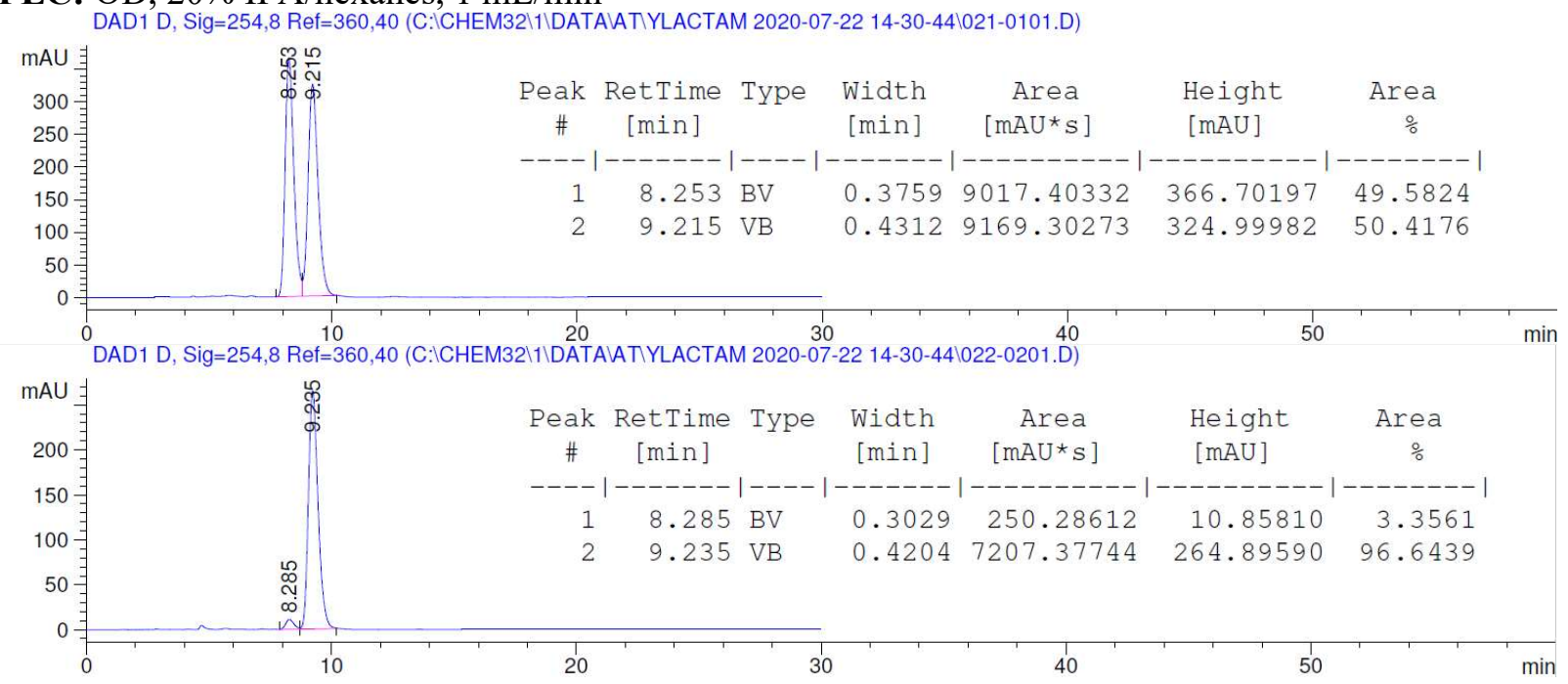

(R)-1,3-bis(4-methoxyphenyl)-3-((4,4,5,5-tetramethyl-1,3,2-dioxaborolan-2-yl)methyl)pyrrolidin-2one (4e)<smiles>COc1ccc(N2CC[C@@](CB3OC(C)(C)C(C)(C)O3)(c3ccc(OC)cc3)C2=O)cc1</smiles>

Prepared according to GP4. Isolated by flash column chromatography, $5 \% \rightarrow 30 \%$ EtOAc/Pentanes. Isolated $78 \mathrm{mg}$ of a white solid ( $85 \%$ yield, $97: 3$ er).

${ }^{1} \mathbf{H}$ NMR $\left(500 \mathrm{MHz}, \mathrm{CDCl}_{3}\right) \delta 7.54(\mathrm{~d}, J=9.2 \mathrm{~Hz}, 2 \mathrm{H}), 7.41(\mathrm{~d}, J=$ $8.9 \mathrm{~Hz}, 2 \mathrm{H}), 6.87$ (d, $J=9.2 \mathrm{~Hz}, 2 \mathrm{H}), 6.83(\mathrm{~d}, J=8.9 \mathrm{~Hz}, 2 \mathrm{H}), 3.78$ (s, 3H), 3.77 (s, 3H), $3.72-3.67$ (m, 2H), 2.69 (ddd, $J=12.8,5.2$, $4.1 \mathrm{~Hz}, 1 \mathrm{H}), 2.48(\mathrm{dt}, J=12.8,8.7 \mathrm{~Hz}, 1 \mathrm{H}), 1.70(\mathrm{~d}, J=15.7 \mathrm{~Hz}$, $1 \mathrm{H}), 1.41(\mathrm{~d}, J=15.7 \mathrm{~Hz}, 1 \mathrm{H}), 1.13(\mathrm{~s}, 6 \mathrm{H}), 1.13(\mathrm{~s}, 6 \mathrm{H})$.

${ }^{13} \mathbf{C}$ NMR $\left(126 \mathrm{MHz}, \mathrm{CDCl}_{3}\right) \delta 176.6,158.3,156.3,135.2,133.3$, 127.4, 121.4, 113.9, 113.6, 83.1, 55.5, 55.2, 50.2, 45.8, 32.8, 24.8, 24.7.

IR $\left(\right.$ ATR, $\left.\mathrm{cm}^{-1}\right) 1673,1508,1374,1329,1302,1254,1035,888,839$

HRMS (DART) m/z: $[\mathrm{M}+\mathrm{H}]^{+}$Calcd for $\mathrm{C}_{25} \mathrm{H}_{33} \mathrm{BNO}_{5} 438.2446$; found 438.2455 MP $80-83{ }^{\circ} \mathrm{C}$

$[\alpha]^{20}{ }_{\mathrm{D}}=+62.53\left(c=0.390, \mathrm{CH}_{2} \mathrm{Cl}_{2}\right)$ 
HPLC: OD, 20\% IPA/hexanes, $1 \mathrm{~mL} / \mathrm{min}$

DAD1 D, Sig=254,8 Ref=360,40 (C:ICHEM32l1IDATALATYLACTAM 2020-07-22 14-30-44l023-0301.D)
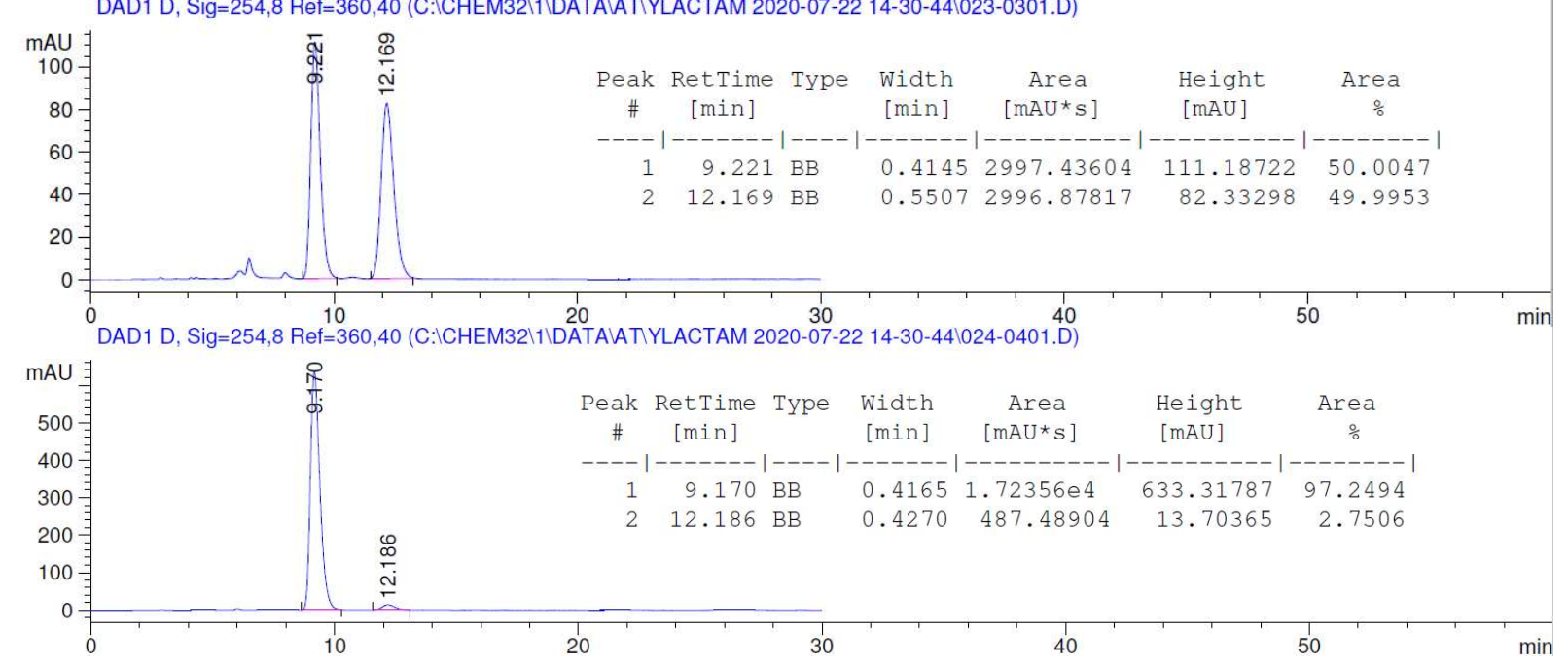

(R)-1-(4-methoxyphenyl)-3-((4,4,5,5-tetramethyl-1,3,2-dioxaborolan-2-yl)methyl)-3-(4(trifluoromethyl)phenyl)pyrrolidin-2-one (4f)

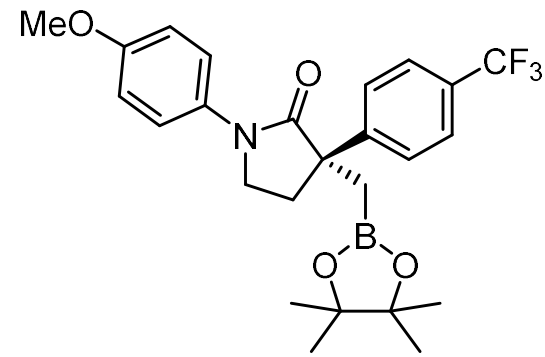

Prepared according to GP4. Isolated by flash column

chromatography, $5 \% \rightarrow 30 \%$ EtOAc/Pentanes. Isolated $62 \mathrm{mg}$ of a white solid (65\% yield, 93:7 er).

${ }^{1} \mathbf{H}$ NMR $\left(500 \mathrm{MHz}, \mathrm{CDCl}_{3}\right) \delta 7.66-7.61(\mathrm{~m}, 2 \mathrm{H}), 7.58-7.47(\mathrm{~m}$, $4 \mathrm{H}), 6.91-6.86(\mathrm{~m}, 2 \mathrm{H}), 3.79(\mathrm{~s}, 3 \mathrm{H}), 3.78-3.66(\mathrm{~m}, 2 \mathrm{H}), 2.70$ (ddd, $J=13.0,6.8,3.2 \mathrm{~Hz}, 1 \mathrm{H}), 2.56(\mathrm{dt}, J=13.0,8.3 \mathrm{~Hz}, 1 \mathrm{H}), 1.71$ $(\mathrm{d}, J=15.9 \mathrm{~Hz}, 1 \mathrm{H}), 1.47(\mathrm{~d}, J=15.9 \mathrm{~Hz}, 1 \mathrm{H}), 1.12(\mathrm{~s}, 12 \mathrm{H})$.

${ }^{13}$ C NMR $\left(126 \mathrm{MHz}, \mathrm{CDCl}_{3}\right) \delta 175.7,156.6,147.4(\mathrm{q}, J=1.3 \mathrm{~Hz})$, $132.9,128.9(\mathrm{q}, J=32.5 \mathrm{~Hz}), 126.9,125.2(\mathrm{q}, J=3.8 \mathrm{~Hz}), 124.2(\mathrm{q}$, $J=272.0 \mathrm{~Hz}), 121.6,114.0,83.3,55.5,51.0,45.7,32.6,24.7,24.7$.

${ }^{19}$ F NMR $\left(377 \mathrm{MHz}, \mathrm{CDCl}_{3}\right) \delta-62.51$.

IR $\left(\mathrm{ATR}, \mathrm{cm}^{-1}\right) 2991,1681,1506,1405,1368,1332,1259,1125,1073,837$

HRMS (DART) m/z: $[\mathrm{M}+\mathrm{H}]^{+}$Calcd for $\mathrm{C}_{25} \mathrm{H}_{30} \mathrm{BNO}_{4} \mathrm{~F}_{3} 476.2215$; found 476.2224

MP $82-90{ }^{\circ} \mathrm{C}$

$[\alpha]^{20}{ }_{\mathrm{D}}=+62.53\left(c=0.175, \mathrm{CH}_{2} \mathrm{Cl}_{2}\right)$

HPLC: OD, 5\% IPA/hexanes, $1 \mathrm{~mL} / \mathrm{min}$

DAD1 D, Sig=254,8 Ref=360,40 (C:ICHEM32|11DATAIBM YLACTAM 2020-07-28 17-14-031091-0201.D)

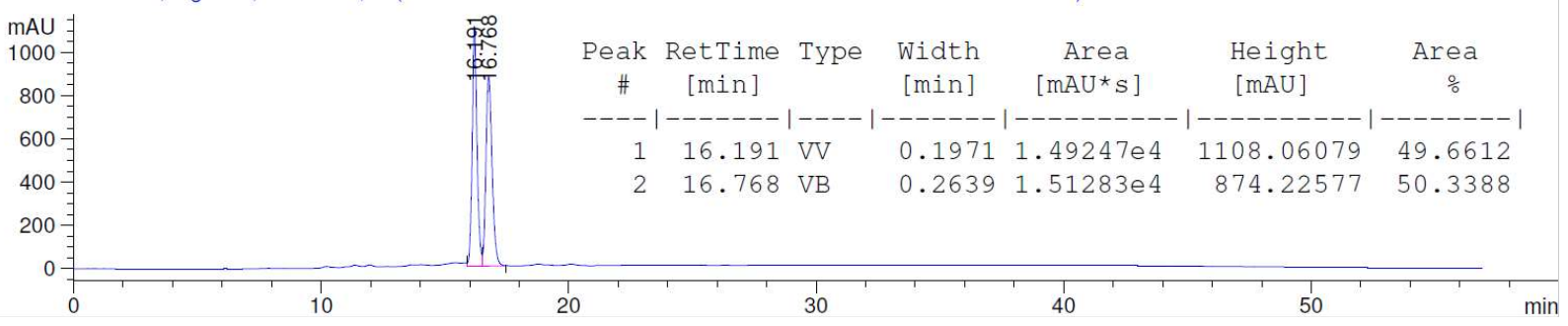


DAD1 D, Sig=254,8 Ref=360,40 (C:ICHEM32\1|DATA\BMYLACTAM 2020-07-28 17-14-03\081-0101.D)

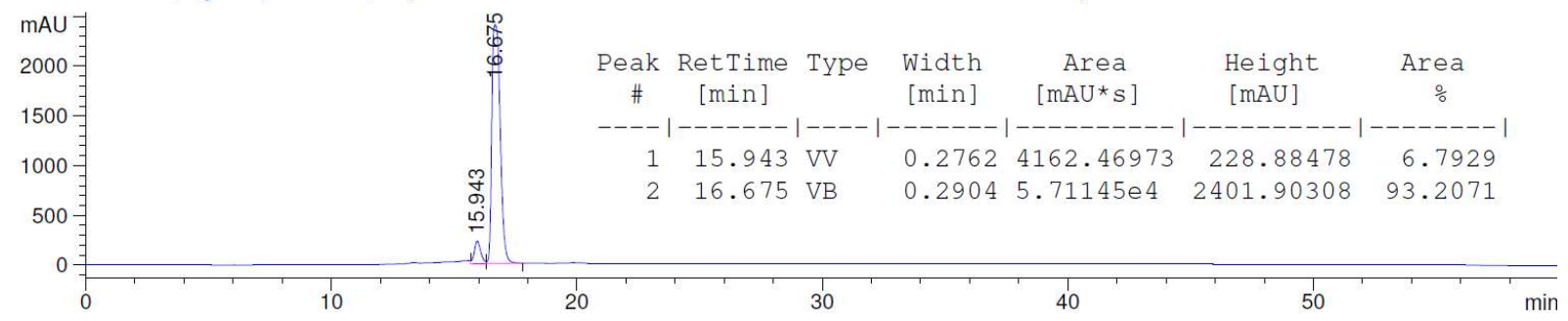




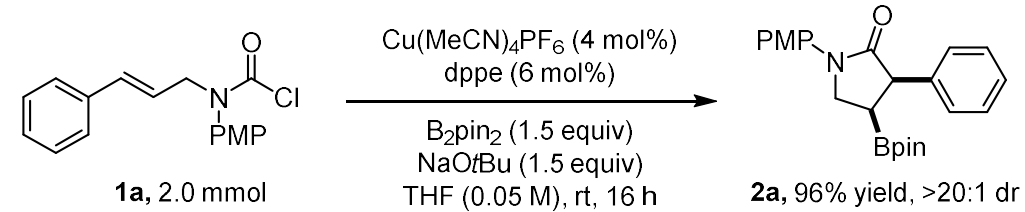

Scheme S9.

An oven dried round bottom flask equipped with a stir bar was flushed with argon. Copper tetrakisacetonitrile hexafluorophoshate $(29.8 \mathrm{mg}, 0.08 \mathrm{mmol}, 4 \mathrm{~mol} \%$ ), dppe (47.8 mg, $0.12 \mathrm{mmol}, 6$ mol\%), and sodium tert-butoxide ( $288 \mathrm{mg}, 3.0 \mathrm{mmol}, 1.5$ equiv) was added followed by THF (10 mL). The catalyst solution was stirred for 5 minutes. $\mathrm{B}_{2} \operatorname{pin}_{2}(762 \mathrm{mg}, 3.0 \mathrm{mmol}, 1.5$ equiv) dissolved in THF $(10 \mathrm{~mL})$ was added and allowed to stir for 5 minutes. Substrate $\mathbf{1 a}(603.5 \mathrm{mg}, 2.0 \mathrm{mmol}, 1.0$ equiv) dissolved in THF $(20 \mathrm{ml})$ was added. The reaction was stirred for $16 \mathrm{~h}$ at room temperature before being filtered over a silica pad with EtOAc. The filtrate was concentrated under reduced pressure and purified by flash column chromatography using $5 \rightarrow 25 \%$ EtOAc/pentanes. Isolated $758 \mathrm{mg}$ of a white solid (96\% yield).

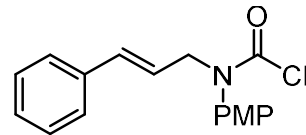

$1 \mathrm{a}, 6.6 \mathrm{mmol}$

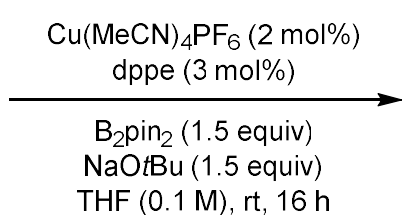

Scheme S10.

An oven dried round bottom flask equipped with a stir bar was flushed with argon. Copper tetrakisacetonitrile hexafluorophoshate $(49.1 \mathrm{mg}, 0.13 \mathrm{mmol}, 2 \mathrm{~mol} \%)$, dppe (78.8 $\mathrm{mg}, 0.19 \mathrm{mmol}, 3$ mol\%), and sodium tert-butoxide (951 mg, $9.9 \mathrm{mmol}, 1.5$ equiv) was added followed by THF (16.5 mL). The catalyst solution was stirred for 5 minutes. $\mathrm{B}_{2} \operatorname{pin}_{2}(2.5 \mathrm{~g}, 9.9 \mathrm{mmol}, 1.5$ equiv) dissolved in THF $(16.5 \mathrm{~mL})$ was added and allowed to stir for 5 minutes. Substrate $1 \mathbf{a}(2.0 \mathrm{~g}, 6.6 \mathrm{mmol}, 1.0$ equiv) dissolved in THF ( $33 \mathrm{ml})$ was added. The reaction was stirred for $16 \mathrm{~h}$ at room temperature before being filtered over a silica pad with EtOAc. The filtrate was concentrated under reduced pressure and purified by flash column chromatography using $5 \rightarrow 25 \%$ EtOAc/pentanes. Isolated $1.94 \mathrm{~g}$ of a white solid (75\% yield).

\section{Oxidation Procedure}

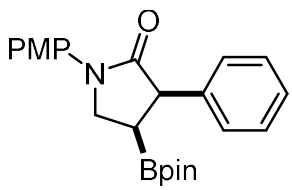

$2 a_{1}>20: 1 d r$

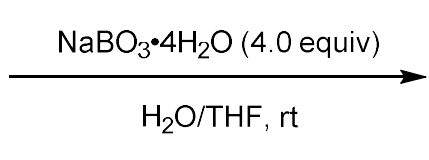

5a, $90 \%$ yield, $>20: 1 d r$

Scheme S11. Oxidation Procedure 
To a solution of $\mathbf{2 a}(78.6 \mathrm{mg}, 0.2 \mathrm{mmol})$ in $\mathrm{THF} / \mathrm{H}_{2} \mathrm{O}(4.0 \mathrm{ml}, 2: 1)$ was added sodium perborate tetrahydrate (4.0 equiv). The reaction was stirred at room temperature for $1 \mathrm{~h}$. The crude mixture was diluted in EtOAc $(5 \mathrm{~mL})$ and washed three times with water $(5 \mathrm{~mL})$. The product was isolated by flash column chromatography $(20 \rightarrow 40 \%$ EtOAc/pentane). Crude NMR identified the product as a $>20: 1 \mathrm{dr}$. The product was isolated as a white solid, $53 \mathrm{mg}$ (90\% yield).

${ }^{1}$ H NMR $\left(500 \mathrm{MHz}, \mathrm{CDCl}_{3}\right) \delta 7.61-7.52(\mathrm{~m}, 2 \mathrm{H}), 7.44-7.36(\mathrm{~m}, 2 \mathrm{H}), 7.37-7.29(\mathrm{~m}, 3 \mathrm{H}), 6.92(\mathrm{~d}, J=$ $9.1 \mathrm{~Hz}, 2 \mathrm{H}), 4.57-4.50(\mathrm{~m}, 1 \mathrm{H}), 4.06(\mathrm{dd}, J=10.7,4.7 \mathrm{~Hz}, 1 \mathrm{H}), 4.02(\mathrm{~d}, J=5.6 \mathrm{~Hz}, 1 \mathrm{H}), 3.81(\mathrm{~s}, 3 \mathrm{H})$, $3.82-3.74(\mathrm{~m}, 1 \mathrm{H}), 1.72(\mathrm{~s}, 1 \mathrm{H})$.

${ }^{13}$ C NMR $\left(126 \mathrm{MHz}, \mathrm{CDCl}_{3}\right) \delta 171.38,156.72,132.73,132.43,130.41,128.89,127.97,121.81,114.11$, 67.21, 55.96, 55.50, 54.95.

IR $\left(\mathrm{ATR}, \mathrm{cm}^{-1}\right) 3398,1672,1516,1431,1316,1256,1111,1034,834,705$

HRMS (DART) m/z: [M+H] $]^{+}$Calcd for $\mathrm{C}_{17} \mathrm{H}_{18} \mathrm{NO}_{3} 284.1281$; found 284.1280

MP $152-154{ }^{\circ} \mathrm{C}$

\section{Vinylation Procedure}

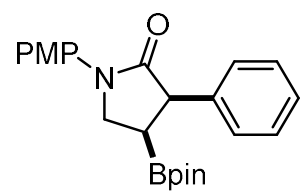

$2 a,>20: 1 d r$

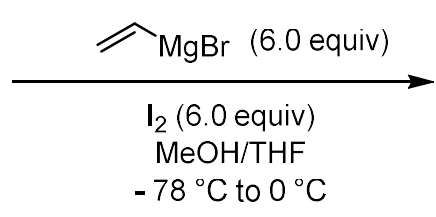

$-78{ }^{\circ} \mathrm{C}$ to $0^{\circ} \mathrm{C}$

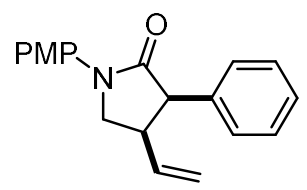

$5 \mathbf{b}, 49 \%$ yield, $>20: 1 d r$

Scheme S12. Vinylation Procedure

In a flame dried vial purged with argon, a solution of $\mathbf{2 a}(78.6 \mathrm{mg}, 0.2 \mathrm{mmol})$ in anhydrous THF (2.0 ml) was cooled to $-78^{\circ} \mathrm{C}$. A solution of vinyl magnesium bromide in THF (6.0 equiv, $1 \mathrm{M}$ solution in THF) was added dropwise and stirred at $-78^{\circ} \mathrm{C}$ for $1.5 \mathrm{~h}$. A solution of $\mathrm{I}_{2}(6.0$ equiv $)$ in $\mathrm{MeOH}(1.6 \mathrm{ml})$ was added dropwise at $-78{ }^{\circ} \mathrm{C}$ and allowed to stir for 30 minutes. The reaction was warmed to $0{ }^{\circ} \mathrm{C}$ and stirred for $1.5 \mathrm{~h}$. The reaction was quenched with $5 \% \mathrm{w} / \mathrm{v} \mathrm{Na}{ }_{2} \mathrm{~S}_{2} \mathrm{O}_{3}$ and extracted with EtOAc. The product was isolated by flash column chromatography $(5 \rightarrow 20 \%$ EtOAc/pentane). Crude NMR identified the product as $>20: 1 \mathrm{dr}$. The product was isolated as a yellow oil, $29 \mathrm{mg}$ (49\% yield).

${ }^{1} \mathbf{H}$ NMR $\left(500 \mathrm{MHz}, \mathrm{CDCl}_{3}\right) \delta 7.66-7.58(\mathrm{~m}, 2 \mathrm{H}), 7.35-7.29(\mathrm{~m}, 2 \mathrm{H}), 7.27-7.23(\mathrm{~m}, 1 \mathrm{H}), 7.18-7.10$ (m, 2H), $6.99-6.89$ (m, 2H), 5.36 (ddd, $J=17.1,10.2,8.6 \mathrm{~Hz}, 1 \mathrm{H}), 5.09$ (ddd, $J=17.1,1.6,1.0 \mathrm{~Hz}, 1 \mathrm{H}$ ), 4.99 (ddd, $J=10.2,1.6,0.7 \mathrm{~Hz}, 1 \mathrm{H}), 4.02-3.92(\mathrm{~m}, 2 \mathrm{H}), 3.82(\mathrm{~s}, 3 \mathrm{H}), 3.79$ (dd, $J=9.8,7.7 \mathrm{~Hz}, 1 \mathrm{H})$, $3.50-3.37(\mathrm{~m}, 1 \mathrm{H})$.

${ }^{13} \mathbf{C}$ NMR $\left(126 \mathrm{MHz}, \mathrm{CDCl}_{3}\right) \delta 173.6,156.8,135.8,135.5,132.3,129.1,128.6,127.2,121.9,117.6$, $114.2,55.5,54.3,52.4,42.1$.

IR $\left(\right.$ ATR, $\left.\mathrm{cm}^{-1}\right)$ 2943, 1686, 1512, 1396, 1333, 1298, 1240, 1031, 834, 710

HRMS (DART) m/z: [M+H] $]^{+}$Calcd for $\mathrm{C}_{19} \mathrm{H}_{20} \mathrm{NO}_{2}$ 294.1489; found 294.1488

\section{Arylation Procedure}




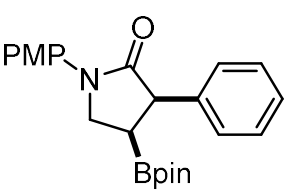

$2 \mathbf{a}_{,}>20: 1 \mathrm{dr}$

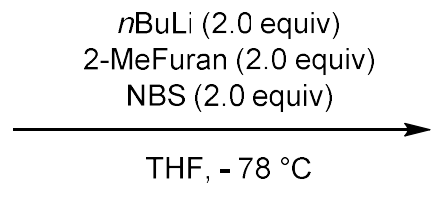

Scheme S13. Arylation Procedure

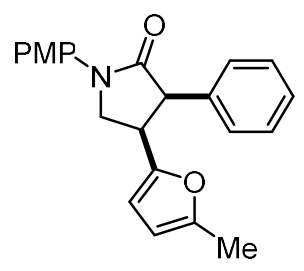

5c, $72 \%$ yield, $>20: 1 d r$

In a flame dried vial purged with argon, a solution of 2-methylfuran ( $35 \mu \mathrm{L}, 0.4 \mathrm{mmol}, 2.0$ equiv) in anhydrous THF $(1.0 \mathrm{~mL})$ was cooled to $-78^{\circ} \mathrm{C}$ and n-butyllithium solution $(2.5 \mathrm{M}, 0.16 \mathrm{~mL}, 0.4 \mathrm{mmol}$, 2.0 equiv) was added dropwise. The solution was stirred for 20 minutes at $-78{ }^{\circ} \mathrm{C}$ then slowly brought up to room temperature, and stirred for an additional $30 \mathrm{~min}$. The solution was then brought back down to $78^{\circ} \mathrm{C}$, and a solution of $\mathbf{2 a}(78.6 \mathrm{mg}, 0.2 \mathrm{mmol}, 1.0$ equiv) in anhydrous THF $(1.0 \mathrm{~mL})$ was added dropwise. The reaction mixture was left to stir at $-78^{\circ} \mathrm{C}$ for $1 \mathrm{~h}$, then a solution of NBS $(71.0 \mathrm{mg}, 0.4$ mmol, 2.0 equiv) in anhydrous THF $(1.0 \mathrm{~mL})$ was added dropwise and left to continue stirring for an additional $1 \mathrm{~h}$ at $-78{ }^{\circ} \mathrm{C}$. The reaction mixture was quenched at $-78{ }^{\circ} \mathrm{C}$ with a $5 \% \mathrm{w} / \mathrm{v}$ solution of $\mathrm{Na}_{2} \mathrm{~S}_{2} \mathrm{O}_{3}$, warmed to room temperature and extracted with EtOAc. The product was isolated by flash column chromatography $(5 \rightarrow 20 \%$ EtOAc/pentane). Crude NMR identified the product as $>20: 1 \mathrm{dr}$. The product was isolated as a white solid, $50 \mathrm{mg}(72 \%$ yield $)$.

${ }^{1} \mathbf{H}$ NMR $\left(500 \mathrm{MHz}, \mathrm{CDCl}_{3}\right) \delta 7.69-7.61(\mathrm{~m}, 2 \mathrm{H}), 7.21-7.14(\mathrm{~m}, 3 \mathrm{H}), 7.01-6.93(\mathrm{~m}, 4 \mathrm{H}), 5.69-5.64$ $(\mathrm{m}, 2 \mathrm{H}), 4.17(\mathrm{~d}, J=8.4 \mathrm{~Hz}, 1 \mathrm{H}), 4.14-4.08(\mathrm{~m}, 2 \mathrm{H}), 4.08-3.99(\mathrm{~m}, 1 \mathrm{H}), 3.83(\mathrm{~s}, 3 \mathrm{H}), 2.07(\mathrm{~d}, J=1.0$ $\mathrm{Hz}, 3 \mathrm{H})$.

${ }^{13}$ C NMR $\left(126 \mathrm{MHz}, \mathrm{CDCl}_{3}\right) \delta 173.0,156.9,151.3,149.7,135.9,132.3,128.9,128.1,127.0,122.1$, $114.2,107.8,105.7,55.5,54.3,51.1,38.1,13.3$.

IR $\left(\mathrm{ATR}, \mathrm{cm}^{-1}\right) 1697,1519,1404,1322,1249,1030,842,797,710,500$

HRMS (DART) m/z: $[\mathrm{M}+\mathrm{H}]^{+}$Calcd for $\mathrm{C}_{22} \mathrm{H}_{22} \mathrm{NO}_{3} 348.1594$; found 348.1599

MP $92-95{ }^{\circ} \mathrm{C}$ 


\section{X-Ray Data for Product 2a}

The sample was prepared by dissolving $\mathbf{2 a}$ in a minimal amount of ethyl acetate, then recrystallizing from pentanes. Data were collected on a Bruker Kappa APEX-DUO diffractometer using monochromated Mo$\mathrm{K} \alpha$ radiation (Bruker Triumph) equipped with a PHOTON II CMOS detector and were measured using a combination of $\phi$ scans and $\omega$ scans. The data were processed using APEX3 and SAINT (Bruker, 2019). Absorption corrections were carried out using SADABS (Bruker, 2019). The structures were solved with SHELXT (Sheldrick, 2015a) and refined using SHELXL-2018 (Sheldrick, 2015b) for full-matrix leastsquares refinement that was based on $F^{2}$. $\mathrm{H}$ atoms were included in calculated positions and allowed to refine in riding-motion approximation with $\mathrm{U} \sim$ iso $\sim$ tied to the carrier atom.

1. Bruker (2007). APEX2, SAINT \& SADABS Bruker AXS Inc., Madison, Wisconsin, USA.

2. Sheldrick, G. M. (2015a). Acta Cryst. A71, 3-8.

3. Sheldrick, G. M. (2015b). Acta Cryst. C71, 3-8

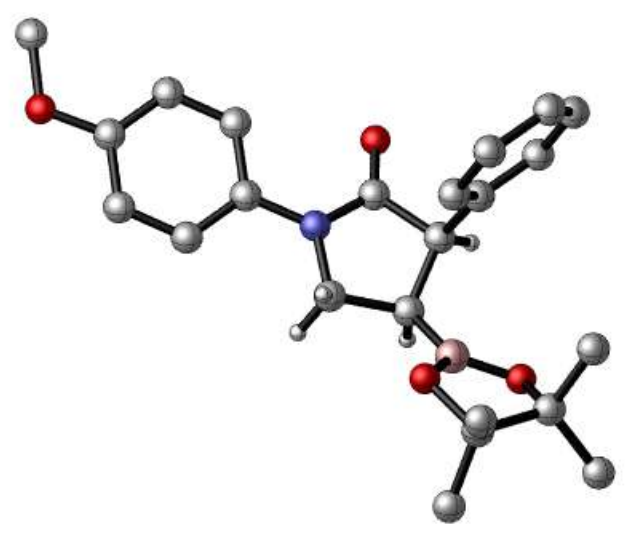

Figure S1. X-ray structure of product 2a. Displacement ellipsoids are shown at the $30 \%$ probability level.

Table 2. Crystal data and structure refinement for d2084a_a (2a).

Identification code

Empirical formula

Formula weight

Temperature

Wavelength

Crystal system

Space group

Unit cell dimensions

Volume

Z

Density (calculated)

Absorption coefficient d2084a a

C23 H28 B N O4

393.27

150(2) K

$0.71073 \AA$

Monoclinic

$\mathrm{P} 2{ }_{1} / \mathrm{c}$

$\mathrm{a}=11.3192(4) \AA$

$\alpha=90^{\circ}$.

$\mathrm{b}=21.8419(7) \AA$

$\beta=112.4960(10)^{\circ}$.

$\mathrm{c}=9.1715(3) \AA$

$\gamma=90^{\circ}$.

2094.95(12) $\AA^{3}$

4

$1.247 \mathrm{Mg} / \mathrm{m}^{3}$

$0.084 \mathrm{~mm}^{-1}$ 
$\mathrm{F}(000)$

Crystal size

Theta range for data collection

Index ranges

Reflections collected

Independent reflections

Completeness to theta $=25.242^{\circ}$

Absorption correction

Max. and min. transmission

Refinement method

Data / restraints / parameters

Goodness-of-fit on $\mathrm{F}^{2}$

Final R indices [I $>2 \operatorname{sigma}(\mathrm{I})]$

$\mathrm{R}$ indices (all data)

Extinction coefficient

Largest diff. peak and hole
840

$0.220 \times 0.100 \times 0.040 \mathrm{~mm}^{3}$

1.865 to $27.495^{\circ}$.

$-14<=\mathrm{h}<=14,-28<=\mathrm{k}<=28,-11<=1<=11$

33056

$4788[\mathrm{R}(\mathrm{int})=0.0748]$

$99.8 \%$

Semi-empirical from equivalents

0.7456 and 0.6963

Full-matrix least-squares on $\mathrm{F}^{2}$

4788 / 0 / 267

1.079

$\mathrm{R} 1=0.0501, \mathrm{wR} 2=0.0988$

$\mathrm{R} 1=0.0747, \mathrm{wR} 2=0.1077$

$\mathrm{n} / \mathrm{a}$

0.295 and -0.234 e. $\AA^{-3}$ 
Table 3. Atomic coordinates ( $\left.\mathrm{x} 10^{4}\right)$ and equivalent isotropic displacement parameters $\left(\AA^{2} \times 10^{3}\right)$ for d2084a_a (2a). U(eq) is defined as one third of the trace of the orthogonalized $U^{i j}$ tensor.

\begin{tabular}{|c|c|c|c|c|}
\hline & $\mathrm{x}$ & $\mathrm{y}$ & $\mathrm{z}$ & $\mathrm{U}(\mathrm{eq})$ \\
\hline $\mathrm{O}(1)$ & $2154(1)$ & $3533(1)$ & $4531(1)$ & $25(1)$ \\
\hline $\mathrm{O}(2)$ & $7284(1)$ & $3107(1)$ & $4669(1)$ & $23(1)$ \\
\hline $\mathrm{O}(3)$ & $6794(1)$ & $4084(1)$ & $3736(1)$ & $25(1)$ \\
\hline $\mathrm{O}(4)$ & $1218(1)$ & $628(1)$ & $5260(1)$ & $32(1)$ \\
\hline $\mathrm{N}(1)$ & $3432(1)$ & $2772(1)$ & $4149(1)$ & $18(1)$ \\
\hline $\mathrm{C}(1)$ & $3105(1)$ & $3364(1)$ & $4322(2)$ & $19(1)$ \\
\hline$C(2)$ & $4178(1)$ & $3785(1)$ & $4306(2)$ & $19(1)$ \\
\hline$C(3)$ & $4885(1)$ & $3392(1)$ & $3499(2)$ & $19(1)$ \\
\hline$C(4)$ & $4568(1)$ & $2724(1)$ & $3757(2)$ & $20(1)$ \\
\hline$C(5)$ & $8482(1)$ & $3372(1)$ & $4697(2)$ & $23(1)$ \\
\hline$C(6)$ & $8188(1)$ & $4072(1)$ & $4579(2)$ & $24(1)$ \\
\hline$C(7)$ & $8675(2)$ & $3112(1)$ & $3266(2)$ & $36(1)$ \\
\hline $\mathrm{C}(8)$ & $9560(2)$ & $3175(1)$ & $6197(2)$ & $34(1)$ \\
\hline$C(9)$ & $8790(2)$ & $4433(1)$ & $3634(2)$ & $38(1)$ \\
\hline$C(10)$ & $8474(2)$ & $4376(1)$ & $6170(2)$ & $36(1)$ \\
\hline $\mathrm{C}(11)$ & 4931(1) & $3977(1)$ & $6003(2)$ & $21(1)$ \\
\hline$C(12)$ & $5791(2)$ & $3582(1)$ & 7091(2) & $25(1)$ \\
\hline$C(13)$ & $6413(2)$ & $3755(1)$ & $8655(2)$ & $34(1)$ \\
\hline$C(14)$ & $6183(2)$ & $4323(1)$ & $9156(2)$ & $43(1)$ \\
\hline$C(15)$ & $5339(2)$ & $4719(1)$ & $8084(2)$ & $42(1)$ \\
\hline$C(16)$ & $4717(2)$ & $4548(1)$ & $6525(2)$ & $31(1)$ \\
\hline$C(17)$ & $2817(1)$ & $2236(1)$ & $4391(2)$ & $18(1)$ \\
\hline$C(18)$ & $3096(1)$ & $1665(1)$ & $3918(2)$ & $22(1)$ \\
\hline$C(19)$ & $2545(2)$ & $1139(1)$ & $4220(2)$ & $25(1)$ \\
\hline$C(20)$ & $1715(1)$ & $1172(1)$ & $5005(2)$ & $23(1)$ \\
\hline$C(21)$ & $1439(1)$ & $1737(1)$ & $5486(2)$ & $22(1)$ \\
\hline$C(22)$ & 1982(1) & $2265(1)$ & $5178(2)$ & $21(1)$ \\
\hline$C(23)$ & $367(2)$ & $669(1)$ & $6076(2)$ & $34(1)$ \\
\hline $\mathrm{B}(1)$ & $6348(2)$ & $3530(1)$ & $3988(2)$ & $20(1)$ \\
\hline
\end{tabular}


Table 4. Bond lengths $[\AA]$ and angles $\left[{ }^{\circ}\right]$ for d2084a_a (2a).

\begin{tabular}{|c|c|}
\hline $\mathrm{O}(1)-\mathrm{C}(1)$ & $1.2207(17)$ \\
\hline $\mathrm{O}(2)-\mathrm{B}(1)$ & $1.364(2)$ \\
\hline $\mathrm{O}(2)-\mathrm{C}(5)$ & $1.4662(17)$ \\
\hline $\mathrm{O}(3)-\mathrm{B}(1)$ & $1.3642(19)$ \\
\hline $\mathrm{O}(3)-\mathrm{C}(6)$ & $1.4678(18)$ \\
\hline $\mathrm{O}(4)-\mathrm{C}(20)$ & $1.3714(18)$ \\
\hline $\mathrm{O}(4)-\mathrm{C}(23)$ & $1.431(2)$ \\
\hline $\mathrm{N}(1)-\mathrm{C}(1)$ & $1.3689(19)$ \\
\hline $\mathrm{N}(1)-\mathrm{C}(17)$ & $1.4222(18)$ \\
\hline $\mathrm{N}(1)-\mathrm{C}(4)$ & $1.4655(18)$ \\
\hline$C(1)-C(2)$ & $1.528(2)$ \\
\hline$C(2)-C(11)$ & $1.520(2)$ \\
\hline$C(2)-C(3)$ & $1.542(2)$ \\
\hline $\mathrm{C}(2)-\mathrm{H}(2 \mathrm{~A})$ & 1.0000 \\
\hline$C(3)-C(4)$ & $1.541(2)$ \\
\hline $\mathrm{C}(3)-\mathrm{B}(1)$ & $1.570(2)$ \\
\hline $\mathrm{C}(3)-\mathrm{H}(3 \mathrm{~A})$ & 1.0000 \\
\hline $\mathrm{C}(4)-\mathrm{H}(4 \mathrm{~A})$ & 0.9900 \\
\hline $\mathrm{C}(4)-\mathrm{H}(4 \mathrm{~B})$ & 0.9900 \\
\hline$C(5)-C(8)$ & $1.511(2)$ \\
\hline$C(5)-C(7)$ & $1.520(2)$ \\
\hline$C(5)-C(6)$ & $1.560(2)$ \\
\hline$C(6)-C(9)$ & $1.513(2)$ \\
\hline$C(6)-C(10)$ & $1.521(2)$ \\
\hline $\mathrm{C}(7)-\mathrm{H}(7 \mathrm{~A})$ & 0.9800 \\
\hline $\mathrm{C}(7)-\mathrm{H}(7 \mathrm{~B})$ & 0.9800 \\
\hline $\mathrm{C}(7)-\mathrm{H}(7 \mathrm{C})$ & 0.9800 \\
\hline $\mathrm{C}(8)-\mathrm{H}(8 \mathrm{~A})$ & 0.9800 \\
\hline $\mathrm{C}(8)-\mathrm{H}(8 \mathrm{~B})$ & 0.9800 \\
\hline $\mathrm{C}(8)-\mathrm{H}(8 \mathrm{C})$ & 0.9800 \\
\hline $\mathrm{C}(9)-\mathrm{H}(9 \mathrm{~A})$ & 0.9800 \\
\hline $\mathrm{C}(9)-\mathrm{H}(9 \mathrm{~B})$ & 0.9800 \\
\hline $\mathrm{C}(9)-\mathrm{H}(9 \mathrm{C})$ & 0.9800 \\
\hline $\mathrm{C}(10)-\mathrm{H}(10 \mathrm{~A})$ & 0.9800 \\
\hline
\end{tabular}




\begin{tabular}{|c|c|}
\hline $\mathrm{C}(10)-\mathrm{H}(10 \mathrm{~B})$ & 0.9800 \\
\hline $\mathrm{C}(10)-\mathrm{H}(10 \mathrm{C})$ & 0.9800 \\
\hline$C(11)-C(16)$ & $1.390(2)$ \\
\hline$C(11)-C(12)$ & $1.394(2)$ \\
\hline$C(12)-C(13)$ & $1.387(2)$ \\
\hline $\mathrm{C}(12)-\mathrm{H}(12 \mathrm{~A})$ & 0.9500 \\
\hline$C(13)-C(14)$ & $1.382(3)$ \\
\hline $\mathrm{C}(13)-\mathrm{H}(13 \mathrm{~A})$ & 0.9500 \\
\hline$C(14)-C(15)$ & $1.382(3)$ \\
\hline $\mathrm{C}(14)-\mathrm{H}(14 \mathrm{~A})$ & 0.9500 \\
\hline$C(15)-C(16)$ & $1.382(3)$ \\
\hline $\mathrm{C}(15)-\mathrm{H}(15 \mathrm{~A})$ & 0.9500 \\
\hline $\mathrm{C}(16)-\mathrm{H}(16 \mathrm{~A})$ & 0.9500 \\
\hline$C(17)-C(22)$ & $1.392(2)$ \\
\hline $\mathrm{C}(17)-\mathrm{C}(18)$ & $1.397(2)$ \\
\hline$C(18)-C(19)$ & $1.384(2)$ \\
\hline $\mathrm{C}(18)-\mathrm{H}(18 \mathrm{~A})$ & 0.9500 \\
\hline$C(19)-C(20)$ & $1.388(2)$ \\
\hline C(19)-H(19A) & 0.9500 \\
\hline$C(20)-C(21)$ & $1.386(2)$ \\
\hline$C(21)-C(22)$ & $1.387(2)$ \\
\hline $\mathrm{C}(21)-\mathrm{H}(21 \mathrm{~A})$ & 0.9500 \\
\hline $\mathrm{C}(22)-\mathrm{H}(22 \mathrm{~A})$ & 0.9500 \\
\hline $\mathrm{C}(23)-\mathrm{H}(23 \mathrm{~A})$ & 0.9800 \\
\hline $\mathrm{C}(23)-\mathrm{H}(23 \mathrm{~B})$ & 0.9800 \\
\hline $\mathrm{C}(23)-\mathrm{H}(23 \mathrm{C})$ & 0.9800 \\
\hline $\mathrm{B}(1)-\mathrm{O}(2)-\mathrm{C}(5)$ & $106.96(12)$ \\
\hline $\mathrm{B}(1)-\mathrm{O}(3)-\mathrm{C}(6)$ & $106.77(11)$ \\
\hline $\mathrm{C}(20)-\mathrm{O}(4)-\mathrm{C}(23)$ & $115.96(12)$ \\
\hline $\mathrm{C}(1)-\mathrm{N}(1)-\mathrm{C}(17)$ & $126.04(12)$ \\
\hline $\mathrm{C}(1)-\mathrm{N}(1)-\mathrm{C}(4)$ & $113.41(11)$ \\
\hline $\mathrm{C}(17)-\mathrm{N}(1)-\mathrm{C}(4)$ & $120.47(11)$ \\
\hline $\mathrm{O}(1)-\mathrm{C}(1)-\mathrm{N}(1)$ & $126.71(14)$ \\
\hline $\mathrm{O}(1)-\mathrm{C}(1)-\mathrm{C}(2)$ & $124.82(13)$ \\
\hline $\mathrm{N}(1)-\mathrm{C}(1)-\mathrm{C}(2)$ & $108.38(12)$ \\
\hline
\end{tabular}




\begin{tabular}{|c|c|}
\hline$C(11)-C(2)-C(1)$ & $107.05(12)$ \\
\hline$C(11)-C(2)-C(3)$ & $117.01(12)$ \\
\hline$C(1)-C(2)-C(3)$ & $103.33(11)$ \\
\hline $\mathrm{C}(11)-\mathrm{C}(2)-\mathrm{H}(2 \mathrm{~A})$ & 109.7 \\
\hline $\mathrm{C}(1)-\mathrm{C}(2)-\mathrm{H}(2 \mathrm{~A})$ & 109.7 \\
\hline $\mathrm{C}(3)-\mathrm{C}(2)-\mathrm{H}(2 \mathrm{~A})$ & 109.7 \\
\hline$C(4)-C(3)-C(2)$ & $104.96(11)$ \\
\hline $\mathrm{C}(4)-\mathrm{C}(3)-\mathrm{B}(1)$ & $114.84(12)$ \\
\hline $\mathrm{C}(2)-\mathrm{C}(3)-\mathrm{B}(1)$ & $116.15(12)$ \\
\hline $\mathrm{C}(4)-\mathrm{C}(3)-\mathrm{H}(3 \mathrm{~A})$ & 106.8 \\
\hline $\mathrm{C}(2)-\mathrm{C}(3)-\mathrm{H}(3 \mathrm{~A})$ & 106.8 \\
\hline $\mathrm{B}(1)-\mathrm{C}(3)-\mathrm{H}(3 \mathrm{~A})$ & 106.8 \\
\hline $\mathrm{N}(1)-\mathrm{C}(4)-\mathrm{C}(3)$ & $104.35(11)$ \\
\hline $\mathrm{N}(1)-\mathrm{C}(4)-\mathrm{H}(4 \mathrm{~A})$ & 110.9 \\
\hline $\mathrm{C}(3)-\mathrm{C}(4)-\mathrm{H}(4 \mathrm{~A})$ & 110.9 \\
\hline $\mathrm{N}(1)-\mathrm{C}(4)-\mathrm{H}(4 \mathrm{~B})$ & 110.9 \\
\hline $\mathrm{C}(3)-\mathrm{C}(4)-\mathrm{H}(4 \mathrm{~B})$ & 110.9 \\
\hline $\mathrm{H}(4 \mathrm{~A})-\mathrm{C}(4)-\mathrm{H}(4 \mathrm{~B})$ & 108.9 \\
\hline $\mathrm{O}(2)-\mathrm{C}(5)-\mathrm{C}(8)$ & $108.80(12)$ \\
\hline $\mathrm{O}(2)-\mathrm{C}(5)-\mathrm{C}(7)$ & $105.95(12)$ \\
\hline$C(8)-C(5)-C(7)$ & $110.35(14)$ \\
\hline $\mathrm{O}(2)-\mathrm{C}(5)-\mathrm{C}(6)$ & $102.39(11)$ \\
\hline$C(8)-C(5)-C(6)$ & $114.78(13)$ \\
\hline$C(7)-C(5)-C(6)$ & $113.77(13)$ \\
\hline $\mathrm{O}(3)-\mathrm{C}(6)-\mathrm{C}(9)$ & $108.58(13)$ \\
\hline $\mathrm{O}(3)-\mathrm{C}(6)-\mathrm{C}(10)$ & $106.80(13)$ \\
\hline$C(9)-C(6)-C(10)$ & $110.23(14)$ \\
\hline $\mathrm{O}(3)-\mathrm{C}(6)-\mathrm{C}(5)$ & $102.22(11)$ \\
\hline$C(9)-C(6)-C(5)$ & $114.78(14)$ \\
\hline$C(10)-C(6)-C(5)$ & $113.52(13)$ \\
\hline $\mathrm{C}(5)-\mathrm{C}(7)-\mathrm{H}(7 \mathrm{~A})$ & 109.5 \\
\hline $\mathrm{C}(5)-\mathrm{C}(7)-\mathrm{H}(7 \mathrm{~B})$ & 109.5 \\
\hline $\mathrm{H}(7 \mathrm{~A})-\mathrm{C}(7)-\mathrm{H}(7 \mathrm{~B})$ & 109.5 \\
\hline $\mathrm{C}(5)-\mathrm{C}(7)-\mathrm{H}(7 \mathrm{C})$ & 109.5 \\
\hline $\mathrm{H}(7 \mathrm{~A})-\mathrm{C}(7)-\mathrm{H}(7 \mathrm{C})$ & 109.5 \\
\hline $\mathrm{H}(7 \mathrm{~B})-\mathrm{C}(7)-\mathrm{H}(7 \mathrm{C})$ & 109.5 \\
\hline
\end{tabular}




\begin{tabular}{|c|c|}
\hline $\mathrm{C}(5)-\mathrm{C}(8)-\mathrm{H}(8 \mathrm{~A})$ & 109.5 \\
\hline $\mathrm{C}(5)-\mathrm{C}(8)-\mathrm{H}(8 \mathrm{~B})$ & 109.5 \\
\hline $\mathrm{H}(8 \mathrm{~A})-\mathrm{C}(8)-\mathrm{H}(8 \mathrm{~B})$ & 109.5 \\
\hline $\mathrm{C}(5)-\mathrm{C}(8)-\mathrm{H}(8 \mathrm{C})$ & 109.5 \\
\hline $\mathrm{H}(8 \mathrm{~A})-\mathrm{C}(8)-\mathrm{H}(8 \mathrm{C})$ & 109.5 \\
\hline $\mathrm{H}(8 \mathrm{~B})-\mathrm{C}(8)-\mathrm{H}(8 \mathrm{C})$ & 109.5 \\
\hline $\mathrm{C}(6)-\mathrm{C}(9)-\mathrm{H}(9 \mathrm{~A})$ & 109.5 \\
\hline $\mathrm{C}(6)-\mathrm{C}(9)-\mathrm{H}(9 \mathrm{~B})$ & 109.5 \\
\hline $\mathrm{H}(9 \mathrm{~A})-\mathrm{C}(9)-\mathrm{H}(9 \mathrm{~B})$ & 109.5 \\
\hline $\mathrm{C}(6)-\mathrm{C}(9)-\mathrm{H}(9 \mathrm{C})$ & 109.5 \\
\hline $\mathrm{H}(9 \mathrm{~A})-\mathrm{C}(9)-\mathrm{H}(9 \mathrm{C})$ & 109.5 \\
\hline $\mathrm{H}(9 \mathrm{~B})-\mathrm{C}(9)-\mathrm{H}(9 \mathrm{C})$ & 109.5 \\
\hline $\mathrm{C}(6)-\mathrm{C}(10)-\mathrm{H}(10 \mathrm{~A})$ & 109.5 \\
\hline $\mathrm{C}(6)-\mathrm{C}(10)-\mathrm{H}(10 \mathrm{~B})$ & 109.5 \\
\hline $\mathrm{H}(10 \mathrm{~A})-\mathrm{C}(10)-\mathrm{H}(10 \mathrm{~B})$ & 109.5 \\
\hline $\mathrm{C}(6)-\mathrm{C}(10)-\mathrm{H}(10 \mathrm{C})$ & 109.5 \\
\hline $\mathrm{H}(10 \mathrm{~A})-\mathrm{C}(10)-\mathrm{H}(10 \mathrm{C})$ & 109.5 \\
\hline $\mathrm{H}(10 \mathrm{~B})-\mathrm{C}(10)-\mathrm{H}(10 \mathrm{C})$ & 109.5 \\
\hline $\mathrm{C}(16)-\mathrm{C}(11)-\mathrm{C}(12)$ & $118.35(15)$ \\
\hline$C(16)-C(11)-C(2)$ & $119.80(14)$ \\
\hline $\mathrm{C}(12)-\mathrm{C}(11)-\mathrm{C}(2)$ & $121.76(13)$ \\
\hline $\mathrm{C}(13)-\mathrm{C}(12)-\mathrm{C}(11)$ & $120.69(15)$ \\
\hline $\mathrm{C}(13)-\mathrm{C}(12)-\mathrm{H}(12 \mathrm{~A})$ & 119.7 \\
\hline $\mathrm{C}(11)-\mathrm{C}(12)-\mathrm{H}(12 \mathrm{~A})$ & 119.7 \\
\hline$C(14)-C(13)-C(12)$ & $120.21(17)$ \\
\hline $\mathrm{C}(14)-\mathrm{C}(13)-\mathrm{H}(13 \mathrm{~A})$ & 119.9 \\
\hline $\mathrm{C}(12)-\mathrm{C}(13)-\mathrm{H}(13 \mathrm{~A})$ & 119.9 \\
\hline$C(15)-C(14)-C(13)$ & $119.52(17)$ \\
\hline$C(15)-C(14)-H(14 A)$ & 120.2 \\
\hline $\mathrm{C}(13)-\mathrm{C}(14)-\mathrm{H}(14 \mathrm{~A})$ & 120.2 \\
\hline$C(14)-C(15)-C(16)$ & $120.40(17)$ \\
\hline $\mathrm{C}(14)-\mathrm{C}(15)-\mathrm{H}(15 \mathrm{~A})$ & 119.8 \\
\hline $\mathrm{C}(16)-\mathrm{C}(15)-\mathrm{H}(15 \mathrm{~A})$ & 119.8 \\
\hline$C(15)-C(16)-C(11)$ & $120.83(16)$ \\
\hline $\mathrm{C}(15)-\mathrm{C}(16)-\mathrm{H}(16 \mathrm{~A})$ & 119.6 \\
\hline $\mathrm{C}(11)-\mathrm{C}(16)-\mathrm{H}(16 \mathrm{~A})$ & 119.6 \\
\hline
\end{tabular}




$\begin{array}{ll}\mathrm{C}(22)-\mathrm{C}(17)-\mathrm{C}(18) & 118.71(13) \\ \mathrm{C}(22)-\mathrm{C}(17)-\mathrm{N}(1) & 120.87(13) \\ \mathrm{C}(18)-\mathrm{C}(17)-\mathrm{N}(1) & 120.33(13) \\ \mathrm{C}(19)-\mathrm{C}(18)-\mathrm{C}(17) & 120.44(14) \\ \mathrm{C}(19)-\mathrm{C}(18)-\mathrm{H}(18 \mathrm{~A}) & 119.8 \\ \mathrm{C}(17)-\mathrm{C}(18)-\mathrm{H}(18 \mathrm{~A}) & 119.8 \\ \mathrm{C}(18)-\mathrm{C}(19)-\mathrm{C}(20) & 120.49(14) \\ \mathrm{C}(18)-\mathrm{C}(19)-\mathrm{H}(19 \mathrm{~A}) & 119.8 \\ \mathrm{C}(20)-\mathrm{C}(19)-\mathrm{H}(19 \mathrm{~A}) & 119.8 \\ \mathrm{O}(4)-\mathrm{C}(20)-\mathrm{C}(21) & 124.06(14) \\ \mathrm{O}(4)-\mathrm{C}(20)-\mathrm{C}(19) & 116.57(14) \\ \mathrm{C}(21)-\mathrm{C}(20)-\mathrm{C}(19) & 119.37(14) \\ \mathrm{C}(20)-\mathrm{C}(21)-\mathrm{C}(22) & 120.37(14) \\ \mathrm{C}(20)-\mathrm{C}(21)-\mathrm{H}(21 \mathrm{~A}) & 119.8 \\ \mathrm{C}(22)-\mathrm{C}(21)-\mathrm{H}(21 \mathrm{~A}) & 119.8 \\ \mathrm{C}(21)-\mathrm{C}(22)-\mathrm{C}(17) & 120.62(14) \\ \mathrm{C}(21)-\mathrm{C}(22)-\mathrm{H}(22 \mathrm{~A}) & 119.7 \\ \mathrm{C}(17)-\mathrm{C}(22)-\mathrm{H}(22 \mathrm{~A}) & 119.7 \\ \mathrm{O}(4)-\mathrm{C}(23)-\mathrm{H}(23 \mathrm{~A}) & 109.5 \\ \mathrm{O}(4)-\mathrm{C}(23)-\mathrm{H}(23 \mathrm{~B}) & 109.5 \\ \mathrm{H}(23 \mathrm{~A})-\mathrm{C}(23)-\mathrm{H}(23 \mathrm{~B}) & 109.5 \\ \mathrm{O}(4)-\mathrm{C}(23)-\mathrm{H}(23 \mathrm{C}) & 109.5 \\ \mathrm{H}(23 \mathrm{~A})-\mathrm{C}(23)-\mathrm{H}(23 \mathrm{C}) & 109.5 \\ \mathrm{H}(23 \mathrm{~B})-\mathrm{C}(23)-\mathrm{H}(23 \mathrm{C}) & 109.5 \\ \mathrm{O}(2)-\mathrm{B}(1)-\mathrm{O}(3) & 113.90(13) \\ \mathrm{O}(2)-\mathrm{B}(1)-\mathrm{C}(3) & 123.38(13) \\ \mathrm{O}(3)-\mathrm{B}(1)-\mathrm{C}(3) & 122.71(13) \\ & \end{array}$

Symmetry transformations used to generate equivalent atoms: 
Table 5. Anisotropic displacement parameters $\left(\AA^{2} \times 10^{3}\right)$ for d2084a_a. The anisotropic displacement factor exponent takes the form: $-2 \pi^{2}\left[h^{2} a^{* 2} U^{11}+\ldots+2 h k a * b * U^{12}\right]$

\begin{tabular}{|c|c|c|c|c|c|c|}
\hline & $\mathrm{U}^{11}$ & $\mathrm{U}^{22}$ & $\mathrm{U}^{33}$ & $\mathrm{U}^{23}$ & $\mathrm{U}^{13}$ & $\mathrm{U}^{12}$ \\
\hline $\mathrm{O}(1)$ & $20(1)$ & $25(1)$ & $34(1)$ & $3(1)$ & $13(1)$ & $4(1)$ \\
\hline $\mathrm{O}(2)$ & $17(1)$ & $23(1)$ & $30(1)$ & $2(1)$ & $10(1)$ & $-2(1)$ \\
\hline $\mathrm{O}(3)$ & $17(1)$ & $24(1)$ & $32(1)$ & $4(1)$ & $7(1)$ & $-2(1)$ \\
\hline $\mathrm{O}(4)$ & $38(1)$ & $24(1)$ & $40(1)$ & $-2(1)$ & $21(1)$ & $-10(1)$ \\
\hline $\mathrm{N}(1)$ & $15(1)$ & $21(1)$ & $20(1)$ & $-1(1)$ & $7(1)$ & $-2(1)$ \\
\hline $\mathrm{C}(1)$ & $16(1)$ & $23(1)$ & $17(1)$ & $3(1)$ & $4(1)$ & $1(1)$ \\
\hline$C(2)$ & $17(1)$ & $20(1)$ & $20(1)$ & $3(1)$ & $6(1)$ & $1(1)$ \\
\hline$C(3)$ & $17(1)$ & $23(1)$ & $18(1)$ & $1(1)$ & $6(1)$ & $-1(1)$ \\
\hline$C(4)$ & $16(1)$ & $23(1)$ & $24(1)$ & $-2(1)$ & $8(1)$ & $-2(1)$ \\
\hline$C(5)$ & $16(1)$ & $28(1)$ & $26(1)$ & $0(1)$ & $9(1)$ & $-3(1)$ \\
\hline$C(6)$ & $16(1)$ & $28(1)$ & $27(1)$ & $0(1)$ & $7(1)$ & $-4(1)$ \\
\hline$C(7)$ & $30(1)$ & $48(1)$ & $37(1)$ & $-9(1)$ & $19(1)$ & $-1(1)$ \\
\hline$C(8)$ & $22(1)$ & $38(1)$ & $37(1)$ & $6(1)$ & $7(1)$ & $2(1)$ \\
\hline$C(9)$ & $27(1)$ & $43(1)$ & $44(1)$ & $9(1)$ & $12(1)$ & $-10(1)$ \\
\hline $\mathrm{C}(10)$ & $29(1)$ & $37(1)$ & $39(1)$ & $-12(1)$ & $12(1)$ & $-8(1)$ \\
\hline $\mathrm{C}(11)$ & $17(1)$ & $24(1)$ & $23(1)$ & $0(1)$ & $10(1)$ & $-4(1)$ \\
\hline $\mathrm{C}(12)$ & $24(1)$ & $28(1)$ & $24(1)$ & $1(1)$ & $11(1)$ & $-1(1)$ \\
\hline$C(13)$ & $29(1)$ & $48(1)$ & $23(1)$ & $3(1)$ & $7(1)$ & $0(1)$ \\
\hline$C(14)$ & $45(1)$ & $55(1)$ & $25(1)$ & $-13(1)$ & $7(1)$ & $-8(1)$ \\
\hline $\mathrm{C}(15)$ & $53(1)$ & $35(1)$ & $39(1)$ & $-14(1)$ & $17(1)$ & $-2(1)$ \\
\hline$C(16)$ & $34(1)$ & $25(1)$ & $33(1)$ & $-3(1)$ & $14(1)$ & $-1(1)$ \\
\hline$C(17)$ & $15(1)$ & $21(1)$ & $17(1)$ & $1(1)$ & $3(1)$ & $-2(1)$ \\
\hline $\mathrm{C}(18)$ & $20(1)$ & $26(1)$ & $21(1)$ & $-2(1)$ & $9(1)$ & $-1(1)$ \\
\hline$C(19)$ & $28(1)$ & $20(1)$ & $27(1)$ & $-3(1)$ & $10(1)$ & $-1(1)$ \\
\hline$C(20)$ & $21(1)$ & $23(1)$ & $22(1)$ & $1(1)$ & $5(1)$ & $-6(1)$ \\
\hline $\mathrm{C}(21)$ & $19(1)$ & $28(1)$ & $22(1)$ & $1(1)$ & $9(1)$ & $-3(1)$ \\
\hline$C(22)$ & $19(1)$ & $21(1)$ & $22(1)$ & $0(1)$ & $6(1)$ & $0(1)$ \\
\hline$C(23)$ & $41(1)$ & $32(1)$ & $35(1)$ & $-4(1)$ & $21(1)$ & $-17(1)$ \\
\hline $\mathrm{B}(1)$ & $20(1)$ & $22(1)$ & $17(1)$ & $-1(1)$ & $9(1)$ & $0(1)$ \\
\hline
\end{tabular}


Table 6. Hydrogen coordinates ( $\left.\mathrm{x} 10^{4}\right)$ and isotropic displacement parameters $\left(\AA^{2} \times 10^{3}\right)$ for d2084a_a (2a).

\begin{tabular}{|c|c|c|c|c|}
\hline & $\mathrm{x}$ & $\mathrm{y}$ & z & $\mathrm{U}(\mathrm{eq})$ \\
\hline $\mathrm{H}(2 \mathrm{~A})$ & 3804 & 4154 & 3648 & 23 \\
\hline $\mathrm{H}(3 \mathrm{~A})$ & 4466 & 3474 & 2341 & 23 \\
\hline $\mathrm{H}(4 \mathrm{~A})$ & 4384 & 2479 & 2788 & 24 \\
\hline $\mathrm{H}(4 \mathrm{~B})$ & 5286 & 2533 & 4632 & 24 \\
\hline $\mathrm{H}(7 \mathrm{~A})$ & 8667 & 2664 & 3307 & 55 \\
\hline $\mathrm{H}(7 \mathrm{~B})$ & 9498 & 3252 & 3267 & 55 \\
\hline $\mathrm{H}(7 \mathrm{C})$ & 7984 & 3254 & 2301 & 55 \\
\hline $\mathrm{H}(8 \mathrm{~A})$ & 9672 & 2731 & 6183 & 51 \\
\hline $\mathrm{H}(8 \mathrm{~B})$ & 9355 & 3287 & 7108 & 51 \\
\hline $\mathrm{H}(8 \mathrm{C})$ & 10352 & 3380 & 6274 & 51 \\
\hline $\mathrm{H}(9 \mathrm{~A})$ & 8542 & 4864 & 3597 & 58 \\
\hline $\mathrm{H}(9 \mathrm{~B})$ & 8496 & 4269 & 2558 & 58 \\
\hline $\mathrm{H}(9 \mathrm{C})$ & 9723 & 4399 & 4134 & 58 \\
\hline $\mathrm{H}(10 \mathrm{~A})$ & 8149 & 4796 & 6008 & 53 \\
\hline $\mathrm{H}(10 \mathrm{~B})$ & 9400 & 4381 & 6770 & 53 \\
\hline $\mathrm{H}(10 \mathrm{C})$ & 8058 & 4145 & 6758 & 53 \\
\hline $\mathrm{H}(12 \mathrm{~A})$ & 5952 & 3190 & 6757 & 30 \\
\hline $\mathrm{H}(13 \mathrm{~A})$ & 6999 & 3482 & 9384 & 41 \\
\hline $\mathrm{H}(14 \mathrm{~A})$ & 6603 & 4440 & 10230 & 52 \\
\hline $\mathrm{H}(15 \mathrm{~A})$ & 5186 & 5112 & 8421 & 51 \\
\hline $\mathrm{H}(16 \mathrm{~A})$ & 4136 & 4824 & 5801 & 37 \\
\hline $\mathrm{H}(18 \mathrm{~A})$ & 3667 & 1637 & 3385 & 26 \\
\hline $\mathrm{H}(19 \mathrm{~A})$ & 2737 & 753 & 3886 & 30 \\
\hline $\mathrm{H}(21 \mathrm{~A})$ & 876 & 1762 & 6029 & 27 \\
\hline $\mathrm{H}(22 \mathrm{~A})$ & 1783 & 2651 & 5506 & 25 \\
\hline $\mathrm{H}(23 \mathrm{~A})$ & 88 & 257 & 6225 & 51 \\
\hline $\mathrm{H}(23 \mathrm{~B})$ & -380 & 915 & 5454 & 51 \\
\hline $\mathrm{H}(23 \mathrm{C})$ & 809 & 862 & 7106 & 51 \\
\hline
\end{tabular}


Table 7. Torsion angles $\left[^{\circ}\right]$ for $\mathrm{d} 2084 \mathrm{a} \_\mathrm{a}$ (2a).

\begin{tabular}{|c|c|}
\hline $\mathrm{C}(17)-\mathrm{N}(1)-\mathrm{C}(1)-\mathrm{O}(1)$ & $-9.0(2)$ \\
\hline $\mathrm{C}(4)-\mathrm{N}(1)-\mathrm{C}(1)-\mathrm{O}(1)$ & $174.22(14)$ \\
\hline $\mathrm{C}(17)-\mathrm{N}(1)-\mathrm{C}(1)-\mathrm{C}(2)$ & $167.66(12)$ \\
\hline $\mathrm{C}(4)-\mathrm{N}(1)-\mathrm{C}(1)-\mathrm{C}(2)$ & $-9.08(16)$ \\
\hline $\mathrm{O}(1)-\mathrm{C}(1)-\mathrm{C}(2)-\mathrm{C}(11)$ & $73.01(18)$ \\
\hline $\mathrm{N}(1)-\mathrm{C}(1)-\mathrm{C}(2)-\mathrm{C}(11)$ & $-103.76(13)$ \\
\hline $\mathrm{O}(1)-\mathrm{C}(1)-\mathrm{C}(2)-\mathrm{C}(3)$ & $-162.87(14)$ \\
\hline $\mathrm{N}(1)-\mathrm{C}(1)-\mathrm{C}(2)-\mathrm{C}(3)$ & $20.35(15)$ \\
\hline $\mathrm{C}(11)-\mathrm{C}(2)-\mathrm{C}(3)-\mathrm{C}(4)$ & $93.95(14)$ \\
\hline $\mathrm{C}(1)-\mathrm{C}(2)-\mathrm{C}(3)-\mathrm{C}(4)$ & $-23.37(14)$ \\
\hline $\mathrm{C}(11)-\mathrm{C}(2)-\mathrm{C}(3)-\mathrm{B}(1)$ & $-34.05(18)$ \\
\hline $\mathrm{C}(1)-\mathrm{C}(2)-\mathrm{C}(3)-\mathrm{B}(1)$ & $-151.37(12)$ \\
\hline $\mathrm{C}(1)-\mathrm{N}(1)-\mathrm{C}(4)-\mathrm{C}(3)$ & $-6.27(16)$ \\
\hline $\mathrm{C}(17)-\mathrm{N}(1)-\mathrm{C}(4)-\mathrm{C}(3)$ & $176.79(12)$ \\
\hline $\mathrm{C}(2)-\mathrm{C}(3)-\mathrm{C}(4)-\mathrm{N}(1)$ & $18.60(14)$ \\
\hline $\mathrm{B}(1)-\mathrm{C}(3)-\mathrm{C}(4)-\mathrm{N}(1)$ & $147.40(12)$ \\
\hline $\mathrm{B}(1)-\mathrm{O}(2)-\mathrm{C}(5)-\mathrm{C}(8)$ & $143.86(13)$ \\
\hline $\mathrm{B}(1)-\mathrm{O}(2)-\mathrm{C}(5)-\mathrm{C}(7)$ & $-97.49(14)$ \\
\hline $\mathrm{B}(1)-\mathrm{O}(2)-\mathrm{C}(5)-\mathrm{C}(6)$ & $21.98(15)$ \\
\hline $\mathrm{B}(1)-\mathrm{O}(3)-\mathrm{C}(6)-\mathrm{C}(9)$ & $145.06(14)$ \\
\hline $\mathrm{B}(1)-\mathrm{O}(3)-\mathrm{C}(6)-\mathrm{C}(10)$ & $-96.08(14)$ \\
\hline $\mathrm{B}(1)-\mathrm{O}(3)-\mathrm{C}(6)-\mathrm{C}(5)$ & $23.39(15)$ \\
\hline $\mathrm{O}(2)-\mathrm{C}(5)-\mathrm{C}(6)-\mathrm{O}(3)$ & $-27.26(14)$ \\
\hline $\mathrm{C}(8)-\mathrm{C}(5)-\mathrm{C}(6)-\mathrm{O}(3)$ & $-144.95(13)$ \\
\hline $\mathrm{C}(7)-\mathrm{C}(5)-\mathrm{C}(6)-\mathrm{O}(3)$ & $86.59(15)$ \\
\hline $\mathrm{O}(2)-\mathrm{C}(5)-\mathrm{C}(6)-\mathrm{C}(9)$ & $-144.57(13)$ \\
\hline$C(8)-C(5)-C(6)-C(9)$ & $97.73(17)$ \\
\hline $\mathrm{C}(7)-\mathrm{C}(5)-\mathrm{C}(6)-\mathrm{C}(9)$ & $-30.73(19)$ \\
\hline $\mathrm{O}(2)-\mathrm{C}(5)-\mathrm{C}(6)-\mathrm{C}(10)$ & $87.38(14)$ \\
\hline$C(8)-C(5)-C(6)-C(10)$ & $-30.32(18)$ \\
\hline$C(7)-C(5)-C(6)-C(10)$ & $-158.78(14)$ \\
\hline$C(1)-C(2)-C(11)-C(16)$ & $-100.20(16)$ \\
\hline$C(3)-C(2)-C(11)-C(16)$ & $144.52(14)$ \\
\hline $\mathrm{C}(1)-\mathrm{C}(2)-\mathrm{C}(11)-\mathrm{C}(12)$ & $76.21(17)$ \\
\hline
\end{tabular}




\begin{tabular}{|c|c|}
\hline $\mathrm{C}(3)-\mathrm{C}(2)-\mathrm{C}(11)-\mathrm{C}(12)$ & $-39.07(19)$ \\
\hline$C(16)-C(11)-C(12)-C(13)$ & $0.1(2)$ \\
\hline $\mathrm{C}(2)-\mathrm{C}(11)-\mathrm{C}(12)-\mathrm{C}(13)$ & $-176.32(14)$ \\
\hline $\mathrm{C}(11)-\mathrm{C}(12)-\mathrm{C}(13)-\mathrm{C}(14)$ & $0.2(3)$ \\
\hline $\mathrm{C}(12)-\mathrm{C}(13)-\mathrm{C}(14)-\mathrm{C}(15)$ & $-0.7(3)$ \\
\hline $\mathrm{C}(13)-\mathrm{C}(14)-\mathrm{C}(15)-\mathrm{C}(16)$ & $0.7(3)$ \\
\hline $\mathrm{C}(14)-\mathrm{C}(15)-\mathrm{C}(16)-\mathrm{C}(11)$ & $-0.3(3)$ \\
\hline $\mathrm{C}(12)-\mathrm{C}(11)-\mathrm{C}(16)-\mathrm{C}(15)$ & $-0.1(2)$ \\
\hline $\mathrm{C}(2)-\mathrm{C}(11)-\mathrm{C}(16)-\mathrm{C}(15)$ & $176.43(16)$ \\
\hline $\mathrm{C}(1)-\mathrm{N}(1)-\mathrm{C}(17)-\mathrm{C}(22)$ & $-14.6(2)$ \\
\hline $\mathrm{C}(4)-\mathrm{N}(1)-\mathrm{C}(17)-\mathrm{C}(22)$ & $161.91(13)$ \\
\hline $\mathrm{C}(1)-\mathrm{N}(1)-\mathrm{C}(17)-\mathrm{C}(18)$ & $168.95(14)$ \\
\hline $\mathrm{C}(4)-\mathrm{N}(1)-\mathrm{C}(17)-\mathrm{C}(18)$ & $-14.5(2)$ \\
\hline $\mathrm{C}(22)-\mathrm{C}(17)-\mathrm{C}(18)-\mathrm{C}(19)$ & $0.4(2)$ \\
\hline $\mathrm{N}(1)-\mathrm{C}(17)-\mathrm{C}(18)-\mathrm{C}(19)$ & $176.89(13)$ \\
\hline $\mathrm{C}(17)-\mathrm{C}(18)-\mathrm{C}(19)-\mathrm{C}(20)$ & $-0.4(2)$ \\
\hline $\mathrm{C}(23)-\mathrm{O}(4)-\mathrm{C}(20)-\mathrm{C}(21)$ & $0.0(2)$ \\
\hline $\mathrm{C}(23)-\mathrm{O}(4)-\mathrm{C}(20)-\mathrm{C}(19)$ & $179.81(14)$ \\
\hline $\mathrm{C}(18)-\mathrm{C}(19)-\mathrm{C}(20)-\mathrm{O}(4)$ & $-179.73(14)$ \\
\hline$C(18)-C(19)-C(20)-C(21)$ & $0.1(2)$ \\
\hline $\mathrm{O}(4)-\mathrm{C}(20)-\mathrm{C}(21)-\mathrm{C}(22)$ & $-179.88(14)$ \\
\hline $\mathrm{C}(19)-\mathrm{C}(20)-\mathrm{C}(21)-\mathrm{C}(22)$ & $0.4(2)$ \\
\hline$C(20)-C(21)-C(22)-C(17)$ & $-0.4(2)$ \\
\hline $\mathrm{C}(18)-\mathrm{C}(17)-\mathrm{C}(22)-\mathrm{C}(21)$ & $0.0(2)$ \\
\hline $\mathrm{N}(1)-\mathrm{C}(17)-\mathrm{C}(22)-\mathrm{C}(21)$ & $-176.46(13)$ \\
\hline $\mathrm{C}(5)-\mathrm{O}(2)-\mathrm{B}(1)-\mathrm{O}(3)$ & $-8.16(17)$ \\
\hline $\mathrm{C}(5)-\mathrm{O}(2)-\mathrm{B}(1)-\mathrm{C}(3)$ & $170.55(13)$ \\
\hline $\mathrm{C}(6)-\mathrm{O}(3)-\mathrm{B}(1)-\mathrm{O}(2)$ & $-10.72(17)$ \\
\hline $\mathrm{C}(6)-\mathrm{O}(3)-\mathrm{B}(1)-\mathrm{C}(3)$ & $170.56(13)$ \\
\hline $\mathrm{C}(4)-\mathrm{C}(3)-\mathrm{B}(1)-\mathrm{O}(2)$ & $-3.4(2)$ \\
\hline $\mathrm{C}(2)-\mathrm{C}(3)-\mathrm{B}(1)-\mathrm{O}(2)$ & $119.53(16)$ \\
\hline $\mathrm{C}(4)-\mathrm{C}(3)-\mathrm{B}(1)-\mathrm{O}(3)$ & $175.15(13)$ \\
\hline $\mathrm{C}(2)-\mathrm{C}(3)-\mathrm{B}(1)-\mathrm{O}(3)$ & $-61.87(19)$ \\
\hline
\end{tabular}

Symmetry transformations used to generate equivalent atoms: 


\section{X-Ray Data for Product 4a}

The sample was prepared by dissolving 4a in a minimal amount of ethyl acetate, then recrystallizing from pentanes. Data were collected on a Bruker Kappa APEX-DUO diffractometer using CuK $\alpha$ radiation from an Incoatec I $\mu$ S source with multi-layer optics and a PHOTON II CMOS detector and were measured using a combination of $\phi$ scans and $\omega$ scans. The data were processed using APEX3 and SAINT (Bruker, 2019). Absorption corrections were carried out using SADABS (Bruker, 2019). The structures were solved with SHELXT (Sheldrick, 2015a) and refined using SHELXL-2018 (Sheldrick, 2015b) for fullmatrix least-squares refinement that was based on $F^{2}$. H atoms were included in calculated positions and allowed to refine in riding-motion approximation with $\mathrm{U} \sim \mathrm{iso} \sim$ tied to the carrier atom.

1. Bruker (2007). APEX2, SAINT \& SADABS Bruker AXS Inc., Madison, Wisconsin, USA.

2. Sheldrick, G. M. (2015a). Acta Cryst. A71, 3-8.

3. Sheldrick, G. M. (2015b). Acta Cryst. C71, 3-8.

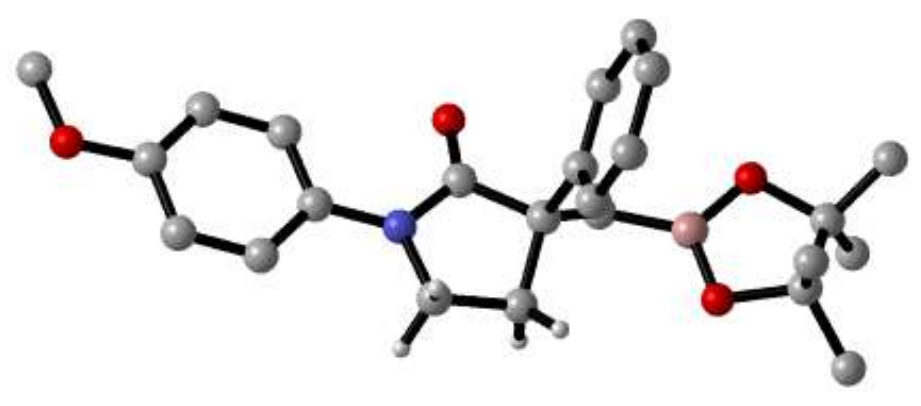

Figure S2. X-ray structure of product 4a. Displacement ellipsoids are shown at the 30\% probability level.

Table 8. Crystal data and structure refinement for d2085_a (4a).

Identification code

Empirical formula

Formula weight

Temperature

Wavelength

Crystal system

Space group

Unit cell dimensions

Volume

Z

Density (calculated)

Absorption coefficient

$\mathrm{F}(000)$

Crystal size d2085_a

C24 H30 B N O4

407.30

150(2) K

\section{$1.54178 \AA$}

Monoclinic

$\mathrm{P} 21$

$\mathrm{a}=9.5706(5) \AA \quad \alpha=90^{\circ}$.

$\mathrm{b}=6.4323(3) \AA$

$\beta=96.009(3)^{\circ}$.

$\mathrm{c}=17.8186(10) \AA$

$\gamma=90^{\circ}$.

1090.9(1) $\AA^{3}$

2

$1.240 \mathrm{Mg} / \mathrm{m}^{3}$

$0.661 \mathrm{~mm}^{-1}$

436

$0.430 \times 0.160 \times 0.150 \mathrm{~mm}^{3}$ 
Theta range for data collection

Index ranges

Reflections collected

Independent reflections

Completeness to theta $=67.679^{\circ}$

Absorption correction

Max. and min. transmission

Refinement method

Data / restraints / parameters

Goodness-of-fit on $\mathrm{F}^{2}$

Final $R$ indices [I $>2 \operatorname{sigma}(\mathrm{I})]$

$\mathrm{R}$ indices (all data)

Absolute structure parameter

Extinction coefficient

Largest diff. peak and hole
2.493 to $68.058^{\circ}$.

$-11<=\mathrm{h}<=11,-7<=\mathrm{k}<=7,-21<=\mathrm{l}<=21$

22737

$3902[\mathrm{R}(\mathrm{int})=0.0681]$

$98.9 \%$

Semi-empirical from equivalents

0.7531 and 0.5825

Full-matrix least-squares on $\mathrm{F}^{2}$

3902 / 1 / 276

1.108

$\mathrm{R} 1=0.0422, \mathrm{wR} 2=0.1066$

$\mathrm{R} 1=0.0493, \mathrm{wR} 2=0.1108$

$-0.16(13)$

$\mathrm{n} / \mathrm{a}$

0.157 and -0.217 e. $\AA^{-3}$ 
Table 9. Atomic coordinates ( $\left.\mathrm{x} 10^{4}\right)$ and equivalent isotropic displacement parameters $\left(\AA^{2} \times 10^{3}\right)$ for d2085_a (4a). U(eq) is defined as one third of the trace of the orthogonalized $U^{i j}$ tensor.

\begin{tabular}{|c|c|c|c|c|}
\hline & $\mathrm{x}$ & $\mathrm{y}$ & $\mathrm{z}$ & $\mathrm{U}(\mathrm{eq})$ \\
\hline $\mathrm{O}(1)$ & $2988(2)$ & $8082(3)$ & $7024(1)$ & $41(1)$ \\
\hline $\mathrm{O}(2)$ & $7290(2)$ & 2804(3) & $7605(1)$ & $34(1)$ \\
\hline $\mathrm{O}(3)$ & $7751(2)$ & $5769(3)$ & $8285(1)$ & $36(1)$ \\
\hline $\mathrm{O}(4)$ & $-3423(2)$ & $7205(3)$ & $5397(1)$ & $36(1)$ \\
\hline $\mathrm{N}(1)$ & $2108(2)$ & $5070(4)$ & 6451(1) & $28(1)$ \\
\hline$C(1)$ & $3070(3)$ & $6236(4)$ & 6895(2) & $29(1)$ \\
\hline$C(2)$ & $4278(3)$ & $4813(4)$ & $7219(2)$ & $29(1)$ \\
\hline$C(3)$ & $4128(3)$ & $2985(5)$ & 6663(2) & $31(1)$ \\
\hline$C(4)$ & $2555(3)$ & 2892(4) & $6405(2)$ & $31(1)$ \\
\hline$C(5)$ & $5695(3)$ & $5951(5)$ & $7238(2)$ & $34(1)$ \\
\hline$C(6)$ & $8312(3)$ & $2216(4)$ & $8241(2)$ & $31(1)$ \\
\hline$C(7)$ & $8904(3)$ & 4358(4) & $8524(2)$ & $32(1)$ \\
\hline$C(8)$ & $9375(4)$ & $758(6)$ & $7955(2)$ & $47(1)$ \\
\hline$C(9)$ & 7473(4) & 1096(6) & $8797(2)$ & $49(1)$ \\
\hline$C(10)$ & $10159(3)$ & $5044(5)$ & $8131(2)$ & $51(1)$ \\
\hline $\mathrm{C}(11)$ & $9232(5)$ & 4533(6) & $9375(2)$ & $58(1)$ \\
\hline$C(12)$ & $3977(3)$ & $4210(4)$ & $8021(2)$ & $27(1)$ \\
\hline$C(13)$ & $3629(3)$ & $2204(5)$ & $8225(2)$ & $32(1)$ \\
\hline$C(14)$ & $3382(3)$ & $1735(5)$ & $8965(2)$ & $37(1)$ \\
\hline$C(15)$ & $3473(3)$ & $3275(5)$ & $9509(2)$ & $41(1)$ \\
\hline$C(16)$ & $3816(3)$ & $5282(5)$ & $9316(2)$ & $42(1)$ \\
\hline $\mathrm{C}(17)$ & $4056(3)$ & $5739(5)$ & $8580(2)$ & $36(1)$ \\
\hline$C(18)$ & $722(3)$ & $5704(4)$ & $6167(2)$ & $27(1)$ \\
\hline$C(19)$ & $-180(3)$ & $4288(5)$ & $5773(2)$ & $34(1)$ \\
\hline $\mathrm{C}(20)$ & $-1549(3)$ & $4845(5)$ & $5523(2)$ & $36(1)$ \\
\hline $\mathrm{C}(21)$ & $-2042(3)$ & $6813(4)$ & $5660(2)$ & $30(1)$ \\
\hline $\mathrm{C}(22)$ & $-1153(3)$ & $8246(5)$ & $6040(2)$ & $35(1)$ \\
\hline$C(23)$ & $222(3)$ & $7694(4)$ & $6287(2)$ & $34(1)$ \\
\hline$C(24)$ & $-3939(3)$ & $9251(5)$ & 5493(2) & $44(1)$ \\
\hline $\mathrm{B}(1)$ & $6927(3)$ & $4828(5)$ & $7712(2)$ & $29(1)$ \\
\hline
\end{tabular}


Table 10. Bond lengths $[\AA]$ and angles $\left[^{\circ}\right]$ for d2085_a (4a).

\begin{tabular}{|c|c|}
\hline $\mathrm{O}(1)-\mathrm{C}(1)$ & $1.214(3)$ \\
\hline $\mathrm{O}(2)-\mathrm{B}(1)$ & $1.366(4)$ \\
\hline $\mathrm{O}(2)-\mathrm{C}(6)$ & $1.467(3)$ \\
\hline $\mathrm{O}(3)-\mathrm{B}(1)$ & $1.364(4)$ \\
\hline $\mathrm{O}(3)-\mathrm{C}(7)$ & $1.458(3)$ \\
\hline $\mathrm{O}(4)-\mathrm{C}(21)$ & $1.378(3)$ \\
\hline $\mathrm{O}(4)-\mathrm{C}(24)$ & $1.422(4)$ \\
\hline $\mathrm{N}(1)-\mathrm{C}(1)$ & $1.372(4)$ \\
\hline $\mathrm{N}(1)-\mathrm{C}(18)$ & $1.429(4)$ \\
\hline $\mathrm{N}(1)-\mathrm{C}(4)$ & $1.470(4)$ \\
\hline $\mathrm{C}(1)-\mathrm{C}(2)$ & $1.538(4)$ \\
\hline$C(2)-C(3)$ & $1.534(4)$ \\
\hline$C(2)-C(12)$ & $1.538(4)$ \\
\hline$C(2)-C(5)$ & $1.539(4)$ \\
\hline $\mathrm{C}(3)-\mathrm{C}(4)$ & $1.529(4)$ \\
\hline $\mathrm{C}(3)-\mathrm{H}(3 \mathrm{~A})$ & 0.9900 \\
\hline $\mathrm{C}(3)-\mathrm{H}(3 \mathrm{~B})$ & 0.9900 \\
\hline $\mathrm{C}(4)-\mathrm{H}(4 \mathrm{~A})$ & 0.9900 \\
\hline $\mathrm{C}(4)-\mathrm{H}(4 \mathrm{~B})$ & 0.9900 \\
\hline $\mathrm{C}(5)-\mathrm{B}(1)$ & $1.555(4)$ \\
\hline $\mathrm{C}(5)-\mathrm{H}(5 \mathrm{~A})$ & 0.9900 \\
\hline $\mathrm{C}(5)-\mathrm{H}(5 \mathrm{~B})$ & 0.9900 \\
\hline $\mathrm{C}(6)-\mathrm{C}(8)$ & $1.510(4)$ \\
\hline$C(6)-C(9)$ & $1.521(4)$ \\
\hline$C(6)-C(7)$ & $1.553(4)$ \\
\hline$C(7)-C(10)$ & $1.518(4)$ \\
\hline$C(7)-C(11)$ & $1.521(5)$ \\
\hline $\mathrm{C}(8)-\mathrm{H}(8 \mathrm{~A})$ & 0.9800 \\
\hline $\mathrm{C}(8)-\mathrm{H}(8 \mathrm{~B})$ & 0.9800 \\
\hline $\mathrm{C}(8)-\mathrm{H}(8 \mathrm{C})$ & 0.9800 \\
\hline $\mathrm{C}(9)-\mathrm{H}(9 \mathrm{~A})$ & 0.9800 \\
\hline $\mathrm{C}(9)-\mathrm{H}(9 \mathrm{~B})$ & 0.9800 \\
\hline $\mathrm{C}(9)-\mathrm{H}(9 \mathrm{C})$ & 0.9800 \\
\hline $\mathrm{C}(10)-\mathrm{H}(10 \mathrm{~A})$ & 0.9800 \\
\hline
\end{tabular}




\begin{tabular}{|c|c|}
\hline $\mathrm{C}(10)-\mathrm{H}(10 \mathrm{~B})$ & 0.9800 \\
\hline $\mathrm{C}(10)-\mathrm{H}(10 \mathrm{C})$ & 0.9800 \\
\hline $\mathrm{C}(11)-\mathrm{H}(11 \mathrm{~A})$ & 0.9800 \\
\hline $\mathrm{C}(11)-\mathrm{H}(11 \mathrm{~B})$ & 0.9800 \\
\hline $\mathrm{C}(11)-\mathrm{H}(11 \mathrm{C})$ & 0.9800 \\
\hline $\mathrm{C}(12)-\mathrm{C}(13)$ & $1.390(4)$ \\
\hline$C(12)-C(17)$ & $1.396(4)$ \\
\hline$C(13)-C(14)$ & $1.396(4)$ \\
\hline $\mathrm{C}(13)-\mathrm{H}(13 \mathrm{~A})$ & 0.9500 \\
\hline$C(14)-C(15)$ & $1.382(5)$ \\
\hline $\mathrm{C}(14)-\mathrm{H}(14 \mathrm{~A})$ & 0.9500 \\
\hline$C(15)-C(16)$ & $1.384(5)$ \\
\hline $\mathrm{C}(15)-\mathrm{H}(15 \mathrm{~A})$ & 0.9500 \\
\hline$C(16)-C(17)$ & $1.387(5)$ \\
\hline $\mathrm{C}(16)-\mathrm{H}(16 \mathrm{~A})$ & 0.9500 \\
\hline $\mathrm{C}(17)-\mathrm{H}(17 \mathrm{~A})$ & 0.9500 \\
\hline$C(18)-C(23)$ & $1.391(4)$ \\
\hline $\mathrm{C}(18)-\mathrm{C}(19)$ & $1.393(4)$ \\
\hline$C(19)-C(20)$ & $1.386(4)$ \\
\hline C(19)-H(19A) & 0.9500 \\
\hline$C(20)-C(21)$ & $1.382(4)$ \\
\hline $\mathrm{C}(20)-\mathrm{H}(20 \mathrm{~A})$ & 0.9500 \\
\hline$C(21)-C(22)$ & $1.382(4)$ \\
\hline$C(22)-C(23)$ & $1.390(4)$ \\
\hline $\mathrm{C}(22)-\mathrm{H}(22 \mathrm{~A})$ & 0.9500 \\
\hline $\mathrm{C}(23)-\mathrm{H}(23 \mathrm{~A})$ & 0.9500 \\
\hline $\mathrm{C}(24)-\mathrm{H}(24 \mathrm{~A})$ & 0.9800 \\
\hline $\mathrm{C}(24)-\mathrm{H}(24 \mathrm{~B})$ & 0.9800 \\
\hline $\mathrm{C}(24)-\mathrm{H}(24 \mathrm{C})$ & 0.9800 \\
\hline $\mathrm{B}(1)-\mathrm{O}(2)-\mathrm{C}(6)$ & 107.2(2) \\
\hline $\mathrm{B}(1)-\mathrm{O}(3)-\mathrm{C}(7)$ & $107.6(2)$ \\
\hline $\mathrm{C}(21)-\mathrm{O}(4)-\mathrm{C}(24)$ & $117.4(2)$ \\
\hline $\mathrm{C}(1)-\mathrm{N}(1)-\mathrm{C}(18)$ & $126.2(2)$ \\
\hline $\mathrm{C}(1)-\mathrm{N}(1)-\mathrm{C}(4)$ & $111.8(2)$ \\
\hline $\mathrm{C}(18)-\mathrm{N}(1)-\mathrm{C}(4)$ & $121.1(2)$ \\
\hline
\end{tabular}




\begin{tabular}{|c|c|}
\hline $\mathrm{O}(1)-\mathrm{C}(1)-\mathrm{N}(1)$ & $126.3(3)$ \\
\hline $\mathrm{O}(1)-\mathrm{C}(1)-\mathrm{C}(2)$ & $124.9(3)$ \\
\hline $\mathrm{N}(1)-\mathrm{C}(1)-\mathrm{C}(2)$ & $108.9(2)$ \\
\hline$C(3)-C(2)-C(12)$ & $113.2(2)$ \\
\hline$C(3)-C(2)-C(1)$ & $101.4(2)$ \\
\hline $\mathrm{C}(12)-\mathrm{C}(2)-\mathrm{C}(1)$ & $107.0(2)$ \\
\hline$C(3)-C(2)-C(5)$ & $113.7(2)$ \\
\hline$C(12)-C(2)-C(5)$ & $110.4(2)$ \\
\hline $\mathrm{C}(1)-\mathrm{C}(2)-\mathrm{C}(5)$ & $110.6(2)$ \\
\hline$C(4)-C(3)-C(2)$ & $104.3(2)$ \\
\hline $\mathrm{C}(4)-\mathrm{C}(3)-\mathrm{H}(3 \mathrm{~A})$ & 110.9 \\
\hline $\mathrm{C}(2)-\mathrm{C}(3)-\mathrm{H}(3 \mathrm{~A})$ & 110.9 \\
\hline $\mathrm{C}(4)-\mathrm{C}(3)-\mathrm{H}(3 \mathrm{~B})$ & 110.9 \\
\hline $\mathrm{C}(2)-\mathrm{C}(3)-\mathrm{H}(3 \mathrm{~B})$ & 110.9 \\
\hline $\mathrm{H}(3 \mathrm{~A})-\mathrm{C}(3)-\mathrm{H}(3 \mathrm{~B})$ & 108.9 \\
\hline $\mathrm{N}(1)-\mathrm{C}(4)-\mathrm{C}(3)$ & $103.2(2)$ \\
\hline $\mathrm{N}(1)-\mathrm{C}(4)-\mathrm{H}(4 \mathrm{~A})$ & 111.1 \\
\hline $\mathrm{C}(3)-\mathrm{C}(4)-\mathrm{H}(4 \mathrm{~A})$ & 111.1 \\
\hline $\mathrm{N}(1)-\mathrm{C}(4)-\mathrm{H}(4 \mathrm{~B})$ & 111.1 \\
\hline $\mathrm{C}(3)-\mathrm{C}(4)-\mathrm{H}(4 \mathrm{~B})$ & 111.1 \\
\hline $\mathrm{H}(4 \mathrm{~A})-\mathrm{C}(4)-\mathrm{H}(4 \mathrm{~B})$ & 109.1 \\
\hline $\mathrm{C}(2)-\mathrm{C}(5)-\mathrm{B}(1)$ & $114.1(2)$ \\
\hline $\mathrm{C}(2)-\mathrm{C}(5)-\mathrm{H}(5 \mathrm{~A})$ & 108.7 \\
\hline $\mathrm{B}(1)-\mathrm{C}(5)-\mathrm{H}(5 \mathrm{~A})$ & 108.7 \\
\hline $\mathrm{C}(2)-\mathrm{C}(5)-\mathrm{H}(5 \mathrm{~B})$ & 108.7 \\
\hline $\mathrm{B}(1)-\mathrm{C}(5)-\mathrm{H}(5 \mathrm{~B})$ & 108.7 \\
\hline $\mathrm{H}(5 \mathrm{~A})-\mathrm{C}(5)-\mathrm{H}(5 \mathrm{~B})$ & 107.6 \\
\hline $\mathrm{O}(2)-\mathrm{C}(6)-\mathrm{C}(8)$ & $108.6(3)$ \\
\hline $\mathrm{O}(2)-\mathrm{C}(6)-\mathrm{C}(9)$ & $105.7(2)$ \\
\hline$C(8)-C(6)-C(9)$ & $110.2(3)$ \\
\hline $\mathrm{O}(2)-\mathrm{C}(6)-\mathrm{C}(7)$ & $102.3(2)$ \\
\hline$C(8)-C(6)-C(7)$ & 115.1(3) \\
\hline$C(9)-C(6)-C(7)$ & 114.1(3) \\
\hline $\mathrm{O}(3)-\mathrm{C}(7)-\mathrm{C}(10)$ & $107.2(2)$ \\
\hline $\mathrm{O}(3)-\mathrm{C}(7)-\mathrm{C}(11)$ & 108.4(3) \\
\hline $\mathrm{C}(10)-\mathrm{C}(7)-\mathrm{C}(11)$ & $110.4(3)$ \\
\hline
\end{tabular}




\begin{tabular}{|c|c|}
\hline $\mathrm{O}(3)-\mathrm{C}(7)-\mathrm{C}(6)$ & $102.6(2)$ \\
\hline$C(10)-C(7)-C(6)$ & $112.8(3)$ \\
\hline$C(11)-C(7)-C(6)$ & $114.8(3)$ \\
\hline $\mathrm{C}(6)-\mathrm{C}(8)-\mathrm{H}(8 \mathrm{~A})$ & 109.5 \\
\hline $\mathrm{C}(6)-\mathrm{C}(8)-\mathrm{H}(8 \mathrm{~B})$ & 109.5 \\
\hline $\mathrm{H}(8 \mathrm{~A})-\mathrm{C}(8)-\mathrm{H}(8 \mathrm{~B})$ & 109.5 \\
\hline $\mathrm{C}(6)-\mathrm{C}(8)-\mathrm{H}(8 \mathrm{C})$ & 109.5 \\
\hline $\mathrm{H}(8 \mathrm{~A})-\mathrm{C}(8)-\mathrm{H}(8 \mathrm{C})$ & 109.5 \\
\hline $\mathrm{H}(8 \mathrm{~B})-\mathrm{C}(8)-\mathrm{H}(8 \mathrm{C})$ & 109.5 \\
\hline $\mathrm{C}(6)-\mathrm{C}(9)-\mathrm{H}(9 \mathrm{~A})$ & 109.5 \\
\hline $\mathrm{C}(6)-\mathrm{C}(9)-\mathrm{H}(9 \mathrm{~B})$ & 109.5 \\
\hline $\mathrm{H}(9 \mathrm{~A})-\mathrm{C}(9)-\mathrm{H}(9 \mathrm{~B})$ & 109.5 \\
\hline $\mathrm{C}(6)-\mathrm{C}(9)-\mathrm{H}(9 \mathrm{C})$ & 109.5 \\
\hline $\mathrm{H}(9 \mathrm{~A})-\mathrm{C}(9)-\mathrm{H}(9 \mathrm{C})$ & 109.5 \\
\hline $\mathrm{H}(9 \mathrm{~B})-\mathrm{C}(9)-\mathrm{H}(9 \mathrm{C})$ & 109.5 \\
\hline $\mathrm{C}(7)-\mathrm{C}(10)-\mathrm{H}(10 \mathrm{~A})$ & 109.5 \\
\hline $\mathrm{C}(7)-\mathrm{C}(10)-\mathrm{H}(10 \mathrm{~B})$ & 109.5 \\
\hline $\mathrm{H}(10 \mathrm{~A})-\mathrm{C}(10)-\mathrm{H}(10 \mathrm{~B})$ & 109.5 \\
\hline $\mathrm{C}(7)-\mathrm{C}(10)-\mathrm{H}(10 \mathrm{C})$ & 109.5 \\
\hline $\mathrm{H}(10 \mathrm{~A})-\mathrm{C}(10)-\mathrm{H}(10 \mathrm{C})$ & 109.5 \\
\hline $\mathrm{H}(10 \mathrm{~B})-\mathrm{C}(10)-\mathrm{H}(10 \mathrm{C})$ & 109.5 \\
\hline $\mathrm{C}(7)-\mathrm{C}(11)-\mathrm{H}(11 \mathrm{~A})$ & 109.5 \\
\hline $\mathrm{C}(7)-\mathrm{C}(11)-\mathrm{H}(11 \mathrm{~B})$ & 109.5 \\
\hline $\mathrm{H}(11 \mathrm{~A})-\mathrm{C}(11)-\mathrm{H}(11 \mathrm{~B})$ & 109.5 \\
\hline $\mathrm{C}(7)-\mathrm{C}(11)-\mathrm{H}(11 \mathrm{C})$ & 109.5 \\
\hline $\mathrm{H}(11 \mathrm{~A})-\mathrm{C}(11)-\mathrm{H}(11 \mathrm{C})$ & 109.5 \\
\hline $\mathrm{H}(11 \mathrm{~B})-\mathrm{C}(11)-\mathrm{H}(11 \mathrm{C})$ & 109.5 \\
\hline$C(13)-C(12)-C(17)$ & $117.7(3)$ \\
\hline$C(13)-C(12)-C(2)$ & $123.3(2)$ \\
\hline$C(17)-C(12)-C(2)$ & $119.0(3)$ \\
\hline$C(12)-C(13)-C(14)$ & $121.0(3)$ \\
\hline $\mathrm{C}(12)-\mathrm{C}(13)-\mathrm{H}(13 \mathrm{~A})$ & 119.5 \\
\hline $\mathrm{C}(14)-\mathrm{C}(13)-\mathrm{H}(13 \mathrm{~A})$ & 119.5 \\
\hline$C(15)-C(14)-C(13)$ & $120.2(3)$ \\
\hline $\mathrm{C}(15)-\mathrm{C}(14)-\mathrm{H}(14 \mathrm{~A})$ & 119.9 \\
\hline $\mathrm{C}(13)-\mathrm{C}(14)-\mathrm{H}(14 \mathrm{~A})$ & 119.9 \\
\hline
\end{tabular}




\begin{tabular}{|c|c|}
\hline $\mathrm{C}(14)-\mathrm{C}(15)-\mathrm{C}(16)$ & $119.6(3)$ \\
\hline $\mathrm{C}(14)-\mathrm{C}(15)-\mathrm{H}(15 \mathrm{~A})$ & 120.2 \\
\hline$C(16)-C(15)-H(15 A)$ & 120.2 \\
\hline$C(15)-C(16)-C(17)$ & $120.0(3)$ \\
\hline $\mathrm{C}(15)-\mathrm{C}(16)-\mathrm{H}(16 \mathrm{~A})$ & 120.0 \\
\hline $\mathrm{C}(17)-\mathrm{C}(16)-\mathrm{H}(16 \mathrm{~A})$ & 120.0 \\
\hline$C(16)-C(17)-C(12)$ & $121.5(3)$ \\
\hline $\mathrm{C}(16)-\mathrm{C}(17)-\mathrm{H}(17 \mathrm{~A})$ & 119.2 \\
\hline $\mathrm{C}(12)-\mathrm{C}(17)-\mathrm{H}(17 \mathrm{~A})$ & 119.2 \\
\hline $\mathrm{C}(23)-\mathrm{C}(18)-\mathrm{C}(19)$ & $118.3(3)$ \\
\hline $\mathrm{C}(23)-\mathrm{C}(18)-\mathrm{N}(1)$ & $121.9(3)$ \\
\hline $\mathrm{C}(19)-\mathrm{C}(18)-\mathrm{N}(1)$ & $119.7(3)$ \\
\hline $\mathrm{C}(20)-\mathrm{C}(19)-\mathrm{C}(18)$ & $120.5(3)$ \\
\hline $\mathrm{C}(20)-\mathrm{C}(19)-\mathrm{H}(19 \mathrm{~A})$ & 119.7 \\
\hline $\mathrm{C}(18)-\mathrm{C}(19)-\mathrm{H}(19 \mathrm{~A})$ & 119.7 \\
\hline$C(21)-C(20)-C(19)$ & $120.6(3)$ \\
\hline $\mathrm{C}(21)-\mathrm{C}(20)-\mathrm{H}(20 \mathrm{~A})$ & 119.7 \\
\hline$C(19)-C(20)-H(20 A)$ & 119.7 \\
\hline $\mathrm{O}(4)-\mathrm{C}(21)-\mathrm{C}(22)$ & $124.3(3)$ \\
\hline $\mathrm{O}(4)-\mathrm{C}(21)-\mathrm{C}(20)$ & $116.1(3)$ \\
\hline $\mathrm{C}(22)-\mathrm{C}(21)-\mathrm{C}(20)$ & $119.5(3)$ \\
\hline $\mathrm{C}(21)-\mathrm{C}(22)-\mathrm{C}(23)$ & $119.9(3)$ \\
\hline $\mathrm{C}(21)-\mathrm{C}(22)-\mathrm{H}(22 \mathrm{~A})$ & 120.0 \\
\hline $\mathrm{C}(23)-\mathrm{C}(22)-\mathrm{H}(22 \mathrm{~A})$ & 120.0 \\
\hline $\mathrm{C}(22)-\mathrm{C}(23)-\mathrm{C}(18)$ & $121.0(3)$ \\
\hline $\mathrm{C}(22)-\mathrm{C}(23)-\mathrm{H}(23 \mathrm{~A})$ & 119.5 \\
\hline $\mathrm{C}(18)-\mathrm{C}(23)-\mathrm{H}(23 \mathrm{~A})$ & 119.5 \\
\hline $\mathrm{O}(4)-\mathrm{C}(24)-\mathrm{H}(24 \mathrm{~A})$ & 109.5 \\
\hline $\mathrm{O}(4)-\mathrm{C}(24)-\mathrm{H}(24 \mathrm{~B})$ & 109.5 \\
\hline $\mathrm{H}(24 \mathrm{~A})-\mathrm{C}(24)-\mathrm{H}(24 \mathrm{~B})$ & 109.5 \\
\hline $\mathrm{O}(4)-\mathrm{C}(24)-\mathrm{H}(24 \mathrm{C})$ & 109.5 \\
\hline $\mathrm{H}(24 \mathrm{~A})-\mathrm{C}(24)-\mathrm{H}(24 \mathrm{C})$ & 109.5 \\
\hline $\mathrm{H}(24 \mathrm{~B})-\mathrm{C}(24)-\mathrm{H}(24 \mathrm{C})$ & 109.5 \\
\hline $\mathrm{O}(3)-\mathrm{B}(1)-\mathrm{O}(2)$ & $113.1(3)$ \\
\hline $\mathrm{O}(3)-\mathrm{B}(1)-\mathrm{C}(5)$ & $123.1(3)$ \\
\hline $\mathrm{O}(2)-\mathrm{B}(1)-\mathrm{C}(5)$ & $123.8(3)$ \\
\hline
\end{tabular}


Symmetry transformations used to generate equivalent atoms: 
Table 11. Anisotropic displacement parameters $\left(\AA^{2} \times 10^{3}\right)$ for d2085_a (4a). The anisotropic displacement factor exponent takes the form: $-2 \pi^{2}\left[h^{2} a^{* 2} U^{11}+\ldots+2 h k a * b * U^{12}\right]$

\begin{tabular}{|c|c|c|c|c|c|c|}
\hline & $\mathrm{U}^{11}$ & $\mathrm{U}^{22}$ & $\mathrm{U}^{33}$ & $\mathrm{U}^{23}$ & $\mathrm{U}^{13}$ & $\mathrm{U}^{12}$ \\
\hline $\mathrm{O}(1)$ & $36(1)$ & $23(1)$ & $60(1)$ & $-2(1)$ & $-12(1)$ & 1(1) \\
\hline $\mathrm{O}(2)$ & $32(1)$ & $32(1)$ & $36(1)$ & $0(1)$ & $-3(1)$ & $2(1)$ \\
\hline $\mathrm{O}(3)$ & $28(1)$ & $29(1)$ & $50(1)$ & $-2(1)$ & $-3(1)$ & $7(1)$ \\
\hline $\mathrm{O}(4)$ & $25(1)$ & $40(1)$ & $40(1)$ & $-1(1)$ & $-1(1)$ & $4(1)$ \\
\hline $\mathrm{N}(1)$ & $25(1)$ & $26(1)$ & $32(1)$ & $-3(1)$ & $-1(1)$ & $5(1)$ \\
\hline $\mathrm{C}(1)$ & $26(1)$ & $27(2)$ & $33(2)$ & 1(1) & $0(1)$ & $-1(1)$ \\
\hline$C(2)$ & $24(1)$ & $28(1)$ & $34(2)$ & 1(1) & $2(1)$ & $1(1)$ \\
\hline$C(3)$ & $31(2)$ & $32(1)$ & $31(1)$ & $-1(1)$ & $5(1)$ & $5(1)$ \\
\hline $\mathrm{C}(4)$ & $30(2)$ & $27(1)$ & $33(2)$ & $-6(1)$ & $0(1)$ & $5(1)$ \\
\hline$C(5)$ & $28(2)$ & $33(2)$ & $41(2)$ & $7(1)$ & $3(1)$ & $-2(1)$ \\
\hline$C(6)$ & $28(1)$ & $27(1)$ & $36(2)$ & $3(1)$ & $0(1)$ & $2(1)$ \\
\hline$C(7)$ & $26(2)$ & $28(2)$ & $40(2)$ & $-1(1)$ & $-5(1)$ & $2(1)$ \\
\hline $\mathrm{C}(8)$ & $42(2)$ & $37(2)$ & $62(2)$ & $-7(2)$ & $-1(2)$ & $13(2)$ \\
\hline$C(9)$ & $44(2)$ & $48(2)$ & $54(2)$ & $20(2)$ & $5(2)$ & $-6(2)$ \\
\hline $\mathrm{C}(10)$ & $27(2)$ & $38(2)$ & $88(3)$ & $14(2)$ & $3(2)$ & $-3(1)$ \\
\hline $\mathrm{C}(11)$ & $68(3)$ & $57(2)$ & $46(2)$ & $-9(2)$ & $-13(2)$ & $9(2)$ \\
\hline$C(12)$ & $22(1)$ & $28(1)$ & $31(1)$ & $-2(1)$ & $2(1)$ & $3(1)$ \\
\hline$C(13)$ & $30(1)$ & $31(1)$ & $35(2)$ & $-1(1)$ & $6(1)$ & $4(1)$ \\
\hline$C(14)$ & $35(2)$ & $40(2)$ & $38(2)$ & $8(1)$ & $8(1)$ & $5(1)$ \\
\hline$C(15)$ & $37(2)$ & $53(2)$ & $33(2)$ & $-2(2)$ & $6(1)$ & $4(2)$ \\
\hline$C(16)$ & $39(2)$ & $51(2)$ & $36(2)$ & $-13(1)$ & $4(1)$ & $0(1)$ \\
\hline$C(17)$ & $35(2)$ & $32(2)$ & $41(2)$ & $-7(1)$ & $6(1)$ & $-3(1)$ \\
\hline$C(18)$ & $25(1)$ & $29(1)$ & $27(1)$ & 1(1) & $0(1)$ & $1(1)$ \\
\hline$C(19)$ & $32(2)$ & $28(1)$ & $42(2)$ & $-6(1)$ & $-1(1)$ & $2(1)$ \\
\hline$C(20)$ & $29(2)$ & $35(2)$ & $42(2)$ & $-3(1)$ & $-1(1)$ & $-2(1)$ \\
\hline$C(21)$ & $22(1)$ & $36(2)$ & $29(1)$ & $4(1)$ & $2(1)$ & $1(1)$ \\
\hline$C(22)$ & $36(2)$ & $29(1)$ & $39(2)$ & $-4(1)$ & $-2(1)$ & $2(1)$ \\
\hline$C(23)$ & $31(2)$ & $27(2)$ & $42(2)$ & $-4(1)$ & $-6(1)$ & $3(1)$ \\
\hline$C(24)$ & $31(2)$ & $44(2)$ & $56(2)$ & $1(2)$ & $-4(2)$ & $7(1)$ \\
\hline $\mathrm{B}(1)$ & $23(2)$ & $29(2)$ & $34(2)$ & $4(1)$ & $6(1)$ & $-1(1)$ \\
\hline
\end{tabular}


Table 12. Hydrogen coordinates ( $\left.\times 10^{4}\right)$ and isotropic displacement parameters $\left(\AA^{2} \times 10^{3}\right)$ for d2085_a(4a).

\begin{tabular}{|c|c|c|c|c|}
\hline & $\mathrm{x}$ & $\mathrm{y}$ & z & $\mathrm{U}(\mathrm{eq})$ \\
\hline $\mathrm{H}(3 \mathrm{~A})$ & 4676 & 3237 & 6230 & 37 \\
\hline $\mathrm{H}(3 \mathrm{~B})$ & 4455 & 1674 & 6914 & 37 \\
\hline $\mathrm{H}(4 \mathrm{~A})$ & 2381 & 2361 & 5882 & 37 \\
\hline $\mathrm{H}(4 \mathrm{~B})$ & 2059 & 1995 & 6742 & 37 \\
\hline $\mathrm{H}(5 \mathrm{~A})$ & 5591 & 7361 & 7448 & 41 \\
\hline $\mathrm{H}(5 \mathrm{~B})$ & 5938 & 6110 & 6715 & 41 \\
\hline $\mathrm{H}(8 \mathrm{~A})$ & 10117 & 460 & 8363 & 71 \\
\hline $\mathrm{H}(8 \mathrm{~B})$ & 9788 & 1409 & 7533 & 71 \\
\hline $\mathrm{H}(8 \mathrm{C})$ & 8909 & -541 & 7785 & 71 \\
\hline $\mathrm{H}(9 \mathrm{~A})$ & 8113 & 592 & 9224 & 73 \\
\hline $\mathrm{H}(9 \mathrm{~B})$ & 6975 & -85 & 8546 & 73 \\
\hline $\mathrm{H}(9 \mathrm{C})$ & 6792 & 2058 & 8981 & 73 \\
\hline $\mathrm{H}(10 \mathrm{~A})$ & 10958 & 4125 & 8278 & 77 \\
\hline $\mathrm{H}(10 \mathrm{~B})$ & 10409 & 6476 & 8277 & 77 \\
\hline $\mathrm{H}(10 \mathrm{C})$ & 9921 & 4974 & 7583 & 77 \\
\hline $\mathrm{H}(11 \mathrm{~A})$ & 9977 & 3545 & 9549 & 88 \\
\hline $\mathrm{H}(11 \mathrm{~B})$ & 8386 & 4218 & 9619 & 88 \\
\hline $\mathrm{H}(11 \mathrm{C})$ & 9546 & 5950 & 9507 & 88 \\
\hline $\mathrm{H}(13 \mathrm{~A})$ & 3558 & 1136 & 7855 & 38 \\
\hline $\mathrm{H}(14 \mathrm{~A})$ & 3151 & 354 & 9094 & 45 \\
\hline $\mathrm{H}(15 \mathrm{~A})$ & 3301 & 2957 & 10012 & 49 \\
\hline $\mathrm{H}(16 \mathrm{~A})$ & 3888 & 6345 & 9688 & 50 \\
\hline $\mathrm{H}(17 \mathrm{~A})$ & 4281 & 7126 & 8453 & 43 \\
\hline $\mathrm{H}(19 \mathrm{~A})$ & 146 & 2930 & 5675 & 41 \\
\hline $\mathrm{H}(20 \mathrm{~A})$ & -2153 & 3865 & 5255 & 43 \\
\hline $\mathrm{H}(22 \mathrm{~A})$ & -1482 & 9607 & 6131 & 42 \\
\hline $\mathrm{H}(23 \mathrm{~A})$ & 831 & 8691 & 6543 & 41 \\
\hline $\mathrm{H}(24 \mathrm{~A})$ & -4940 & 9307 & 5308 & 66 \\
\hline $\mathrm{H}(24 \mathrm{~B})$ & -3419 & 10235 & 5208 & 66 \\
\hline $\mathrm{H}(24 \mathrm{C})$ & -3816 & 9619 & 6030 & 66 \\
\hline
\end{tabular}


Table 13. Torsion angles $\left[{ }^{\circ}\right]$ for d2085_a (4a).

\begin{tabular}{|c|c|}
\hline $\mathrm{C}(18)-\mathrm{N}(1)-\mathrm{C}(1)-\mathrm{O}(1)$ & $-11.9(5)$ \\
\hline $\mathrm{C}(4)-\mathrm{N}(1)-\mathrm{C}(1)-\mathrm{O}(1)$ & $178.8(3)$ \\
\hline $\mathrm{C}(18)-\mathrm{N}(1)-\mathrm{C}(1)-\mathrm{C}(2)$ & $167.2(2)$ \\
\hline $\mathrm{C}(4)-\mathrm{N}(1)-\mathrm{C}(1)-\mathrm{C}(2)$ & $-2.1(3)$ \\
\hline $\mathrm{O}(1)-\mathrm{C}(1)-\mathrm{C}(2)-\mathrm{C}(3)$ & $-160.0(3)$ \\
\hline $\mathrm{N}(1)-\mathrm{C}(1)-\mathrm{C}(2)-\mathrm{C}(3)$ & $20.9(3)$ \\
\hline $\mathrm{O}(1)-\mathrm{C}(1)-\mathrm{C}(2)-\mathrm{C}(12)$ & $81.3(3)$ \\
\hline $\mathrm{N}(1)-\mathrm{C}(1)-\mathrm{C}(2)-\mathrm{C}(12)$ & $-97.9(3)$ \\
\hline $\mathrm{O}(1)-\mathrm{C}(1)-\mathrm{C}(2)-\mathrm{C}(5)$ & $-39.1(4)$ \\
\hline $\mathrm{N}(1)-\mathrm{C}(1)-\mathrm{C}(2)-\mathrm{C}(5)$ & $141.8(2)$ \\
\hline $\mathrm{C}(12)-\mathrm{C}(2)-\mathrm{C}(3)-\mathrm{C}(4)$ & $83.5(3)$ \\
\hline$C(1)-C(2)-C(3)-C(4)$ & $-30.8(3)$ \\
\hline$C(5)-C(2)-C(3)-C(4)$ & $-149.5(2)$ \\
\hline $\mathrm{C}(1)-\mathrm{N}(1)-\mathrm{C}(4)-\mathrm{C}(3)$ & $-17.8(3)$ \\
\hline $\mathrm{C}(18)-\mathrm{N}(1)-\mathrm{C}(4)-\mathrm{C}(3)$ & $172.2(2)$ \\
\hline $\mathrm{C}(2)-\mathrm{C}(3)-\mathrm{C}(4)-\mathrm{N}(1)$ & $30.2(3)$ \\
\hline $\mathrm{C}(3)-\mathrm{C}(2)-\mathrm{C}(5)-\mathrm{B}(1)$ & $-79.3(3)$ \\
\hline $\mathrm{C}(12)-\mathrm{C}(2)-\mathrm{C}(5)-\mathrm{B}(1)$ & $49.1(3)$ \\
\hline $\mathrm{C}(1)-\mathrm{C}(2)-\mathrm{C}(5)-\mathrm{B}(1)$ & $167.4(3)$ \\
\hline $\mathrm{B}(1)-\mathrm{O}(2)-\mathrm{C}(6)-\mathrm{C}(8)$ & $-144.2(3)$ \\
\hline $\mathrm{B}(1)-\mathrm{O}(2)-\mathrm{C}(6)-\mathrm{C}(9)$ & $97.5(3)$ \\
\hline $\mathrm{B}(1)-\mathrm{O}(2)-\mathrm{C}(6)-\mathrm{C}(7)$ & $-22.1(3)$ \\
\hline $\mathrm{B}(1)-\mathrm{O}(3)-\mathrm{C}(7)-\mathrm{C}(10)$ & $97.4(3)$ \\
\hline $\mathrm{B}(1)-\mathrm{O}(3)-\mathrm{C}(7)-\mathrm{C}(11)$ & $-143.4(3)$ \\
\hline $\mathrm{B}(1)-\mathrm{O}(3)-\mathrm{C}(7)-\mathrm{C}(6)$ & $-21.6(3)$ \\
\hline $\mathrm{O}(2)-\mathrm{C}(6)-\mathrm{C}(7)-\mathrm{O}(3)$ & $26.2(3)$ \\
\hline $\mathrm{C}(8)-\mathrm{C}(6)-\mathrm{C}(7)-\mathrm{O}(3)$ & $143.7(3)$ \\
\hline $\mathrm{C}(9)-\mathrm{C}(6)-\mathrm{C}(7)-\mathrm{O}(3)$ & $-87.5(3)$ \\
\hline $\mathrm{O}(2)-\mathrm{C}(6)-\mathrm{C}(7)-\mathrm{C}(10)$ & $-88.9(3)$ \\
\hline$C(8)-C(6)-C(7)-C(10)$ & $28.7(4)$ \\
\hline$C(9)-C(6)-C(7)-C(10)$ & $157.5(3)$ \\
\hline $\mathrm{O}(2)-\mathrm{C}(6)-\mathrm{C}(7)-\mathrm{C}(11)$ & $143.5(3)$ \\
\hline$C(8)-C(6)-C(7)-C(11)$ & $-99.0(3)$ \\
\hline$C(9)-C(6)-C(7)-C(11)$ & $29.9(4)$ \\
\hline
\end{tabular}




\begin{tabular}{|c|c|}
\hline$C(3)-C(2)-C(12)-C(13)$ & $1.1(4)$ \\
\hline$C(1)-C(2)-C(12)-C(13)$ & $111.9(3)$ \\
\hline$C(5)-C(2)-C(12)-C(13)$ & $-127.7(3)$ \\
\hline$C(3)-C(2)-C(12)-C(17)$ & $-179.1(2)$ \\
\hline$C(1)-C(2)-C(12)-C(17)$ & $-68.2(3)$ \\
\hline$C(5)-C(2)-C(12)-C(17)$ & $52.2(3)$ \\
\hline$C(17)-C(12)-C(13)-C(14)$ & $-0.6(4)$ \\
\hline$C(2)-C(12)-C(13)-C(14)$ & $179.2(3)$ \\
\hline$C(12)-C(13)-C(14)-C(15)$ & $0.4(4)$ \\
\hline$C(13)-C(14)-C(15)-C(16)$ & $-0.3(5)$ \\
\hline$C(14)-C(15)-C(16)-C(17)$ & $0.5(5)$ \\
\hline$C(15)-C(16)-C(17)-C(12)$ & $-0.7(5)$ \\
\hline$C(13)-C(12)-C(17)-C(16)$ & $0.8(4)$ \\
\hline$C(2)-C(12)-C(17)-C(16)$ & $-179.0(3)$ \\
\hline $\mathrm{C}(1)-\mathrm{N}(1)-\mathrm{C}(18)-\mathrm{C}(23)$ & $3.5(4)$ \\
\hline $\mathrm{C}(4)-\mathrm{N}(1)-\mathrm{C}(18)-\mathrm{C}(23)$ & $171.9(3)$ \\
\hline $\mathrm{C}(1)-\mathrm{N}(1)-\mathrm{C}(18)-\mathrm{C}(19)$ & $-174.8(3)$ \\
\hline $\mathrm{C}(4)-\mathrm{N}(1)-\mathrm{C}(18)-\mathrm{C}(19)$ & $-6.4(4)$ \\
\hline $\mathrm{C}(23)-\mathrm{C}(18)-\mathrm{C}(19)-\mathrm{C}(20)$ & $-1.3(4)$ \\
\hline $\mathrm{N}(1)-\mathrm{C}(18)-\mathrm{C}(19)-\mathrm{C}(20)$ & $177.0(3)$ \\
\hline $\mathrm{C}(18)-\mathrm{C}(19)-\mathrm{C}(20)-\mathrm{C}(21)$ & $0.0(5)$ \\
\hline $\mathrm{C}(24)-\mathrm{O}(4)-\mathrm{C}(21)-\mathrm{C}(22)$ & $3.1(4)$ \\
\hline $\mathrm{C}(24)-\mathrm{O}(4)-\mathrm{C}(21)-\mathrm{C}(20)$ & $-176.7(3)$ \\
\hline $\mathrm{C}(19)-\mathrm{C}(20)-\mathrm{C}(21)-\mathrm{O}(4)$ & $-179.2(3)$ \\
\hline$C(19)-C(20)-C(21)-C(22)$ & $1.0(4)$ \\
\hline $\mathrm{O}(4)-\mathrm{C}(21)-\mathrm{C}(22)-\mathrm{C}(23)$ & $179.5(3)$ \\
\hline $\mathrm{C}(20)-\mathrm{C}(21)-\mathrm{C}(22)-\mathrm{C}(23)$ & $-0.7(4)$ \\
\hline $\mathrm{C}(21)-\mathrm{C}(22)-\mathrm{C}(23)-\mathrm{C}(18)$ & $-0.7(5)$ \\
\hline $\mathrm{C}(19)-\mathrm{C}(18)-\mathrm{C}(23)-\mathrm{C}(22)$ & $1.7(4)$ \\
\hline $\mathrm{N}(1)-\mathrm{C}(18)-\mathrm{C}(23)-\mathrm{C}(22)$ & $-176.6(3)$ \\
\hline $\mathrm{C}(7)-\mathrm{O}(3)-\mathrm{B}(1)-\mathrm{O}(2)$ & $8.5(3)$ \\
\hline $\mathrm{C}(7)-\mathrm{O}(3)-\mathrm{B}(1)-\mathrm{C}(5)$ & $-172.0(3)$ \\
\hline $\mathrm{C}(6)-\mathrm{O}(2)-\mathrm{B}(1)-\mathrm{O}(3)$ & $9.7(3)$ \\
\hline $\mathrm{C}(6)-\mathrm{O}(2)-\mathrm{B}(1)-\mathrm{C}(5)$ & $-169.8(3)$ \\
\hline $\mathrm{C}(2)-\mathrm{C}(5)-\mathrm{B}(1)-\mathrm{O}(3)$ & $-125.5(3)$ \\
\hline $\mathrm{C}(2)-\mathrm{C}(5)-\mathrm{B}(1)-\mathrm{O}(2)$ & $53.9(4)$ \\
\hline
\end{tabular}


Symmetry transformations used to generate equivalent atoms: 
NOESY Spectra for Product $2 q$

NOESY Correlation: $\mathbf{2 q}$ (major diastereomer, $500 \mathrm{MHz}, \mathrm{CDCl}_{3}$ )

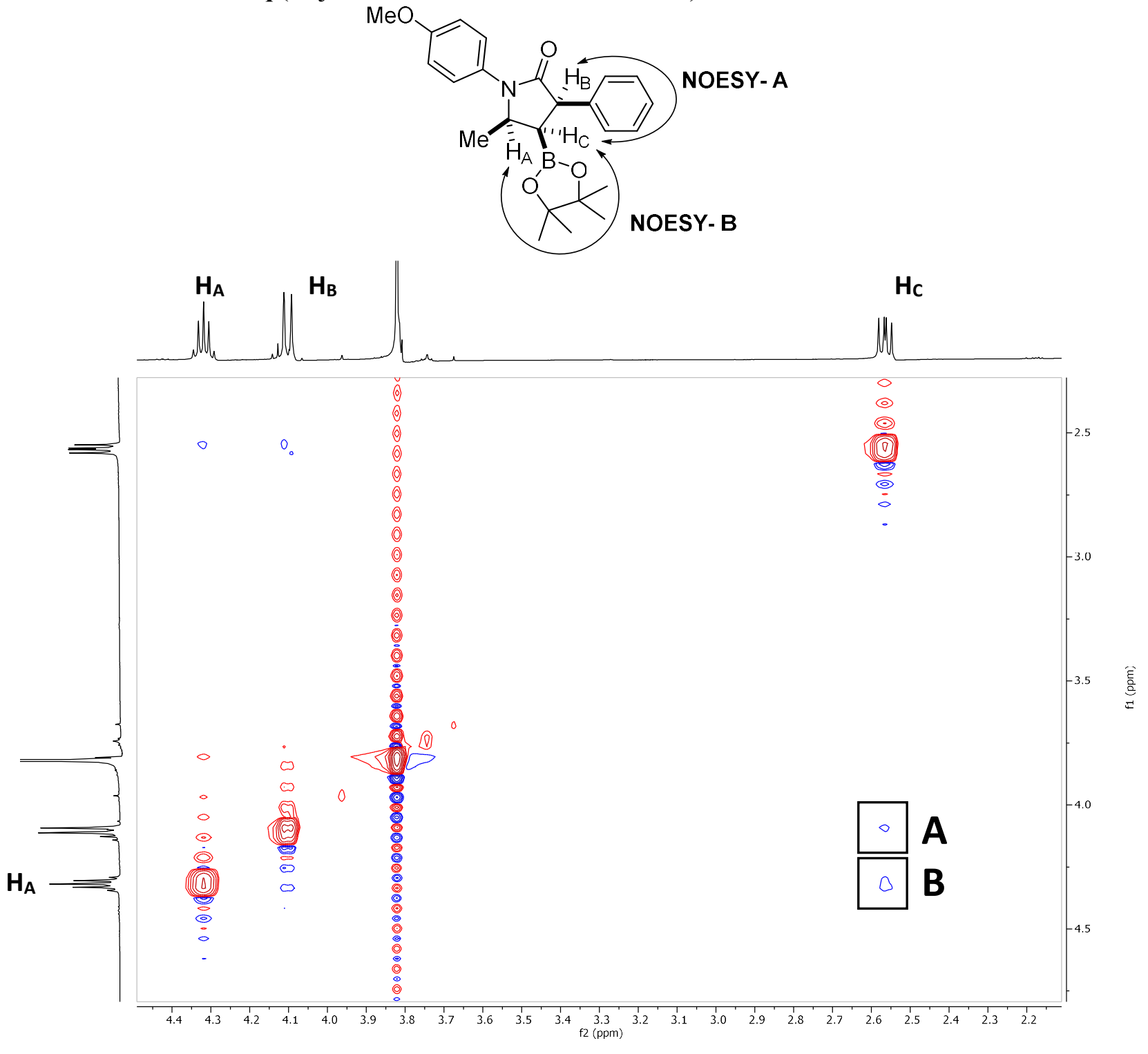


NOESY Correlation: 2q (minor diastereomer, $500 \mathrm{MHz}, \mathrm{CDCl}_{3}$ )

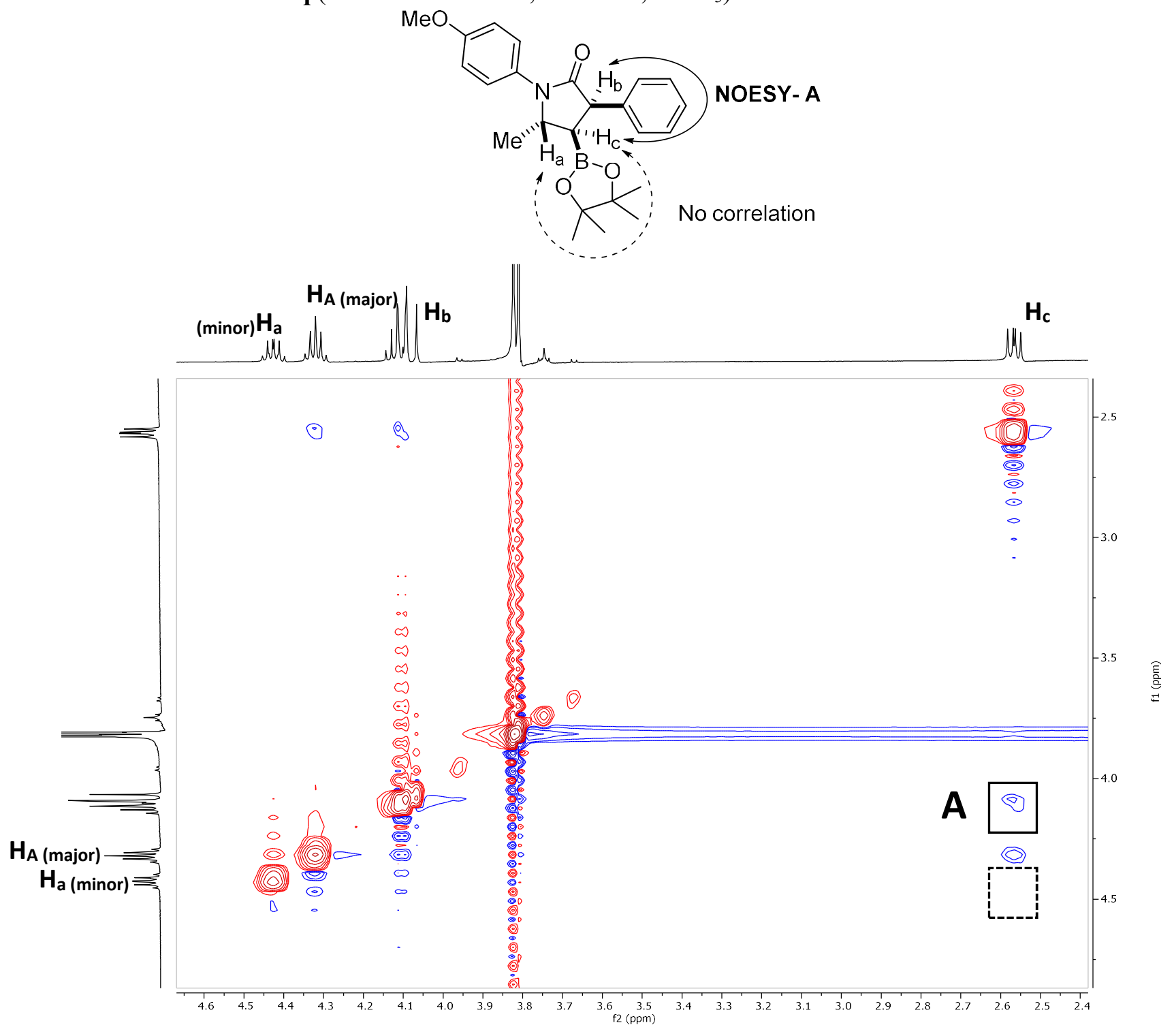


NOESY Spectra for Product 5a

NOESY Correlation: 5a (major diastereomer, $500 \mathrm{MHz}, \mathrm{CDCl}_{3}$ )

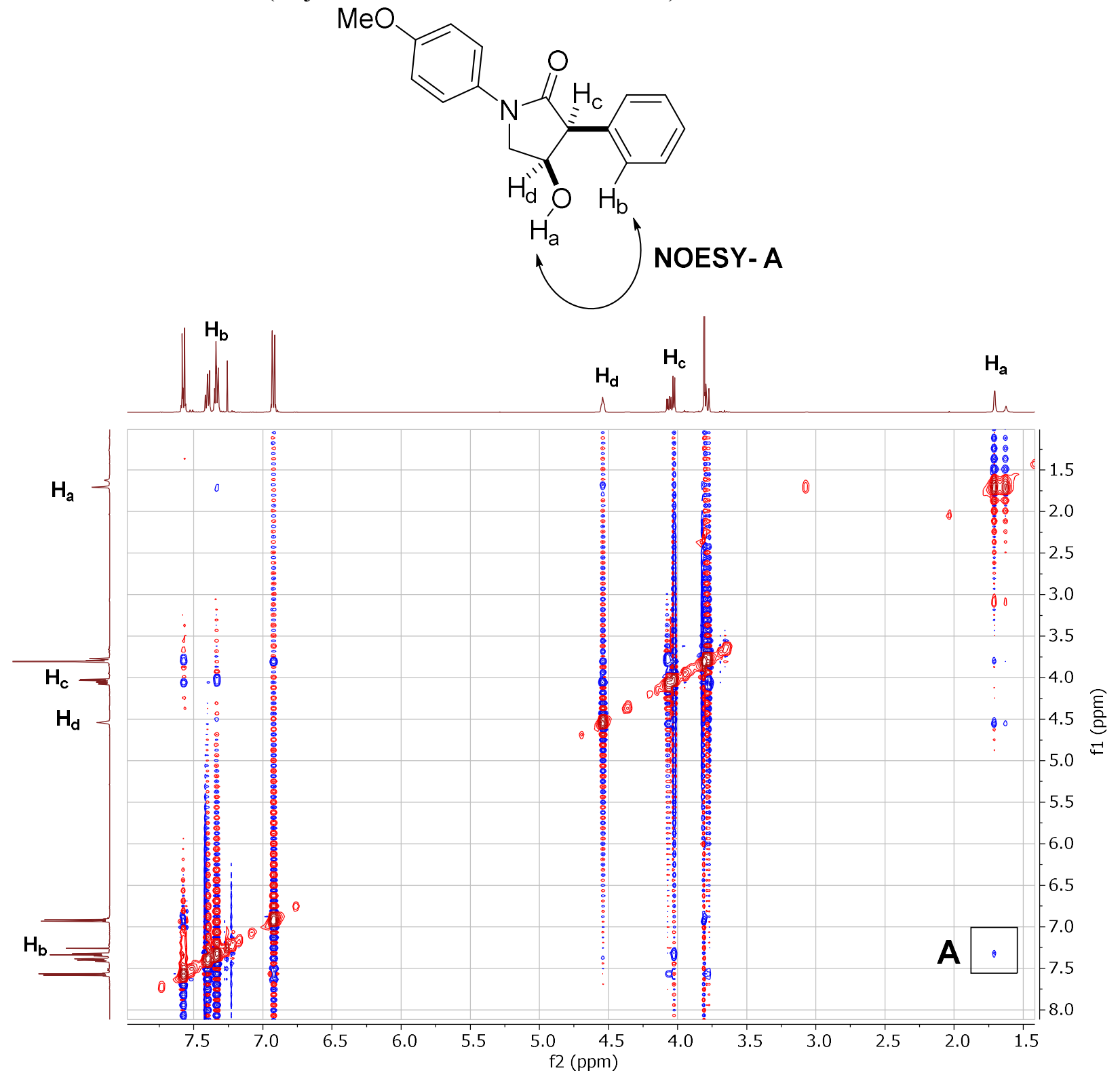




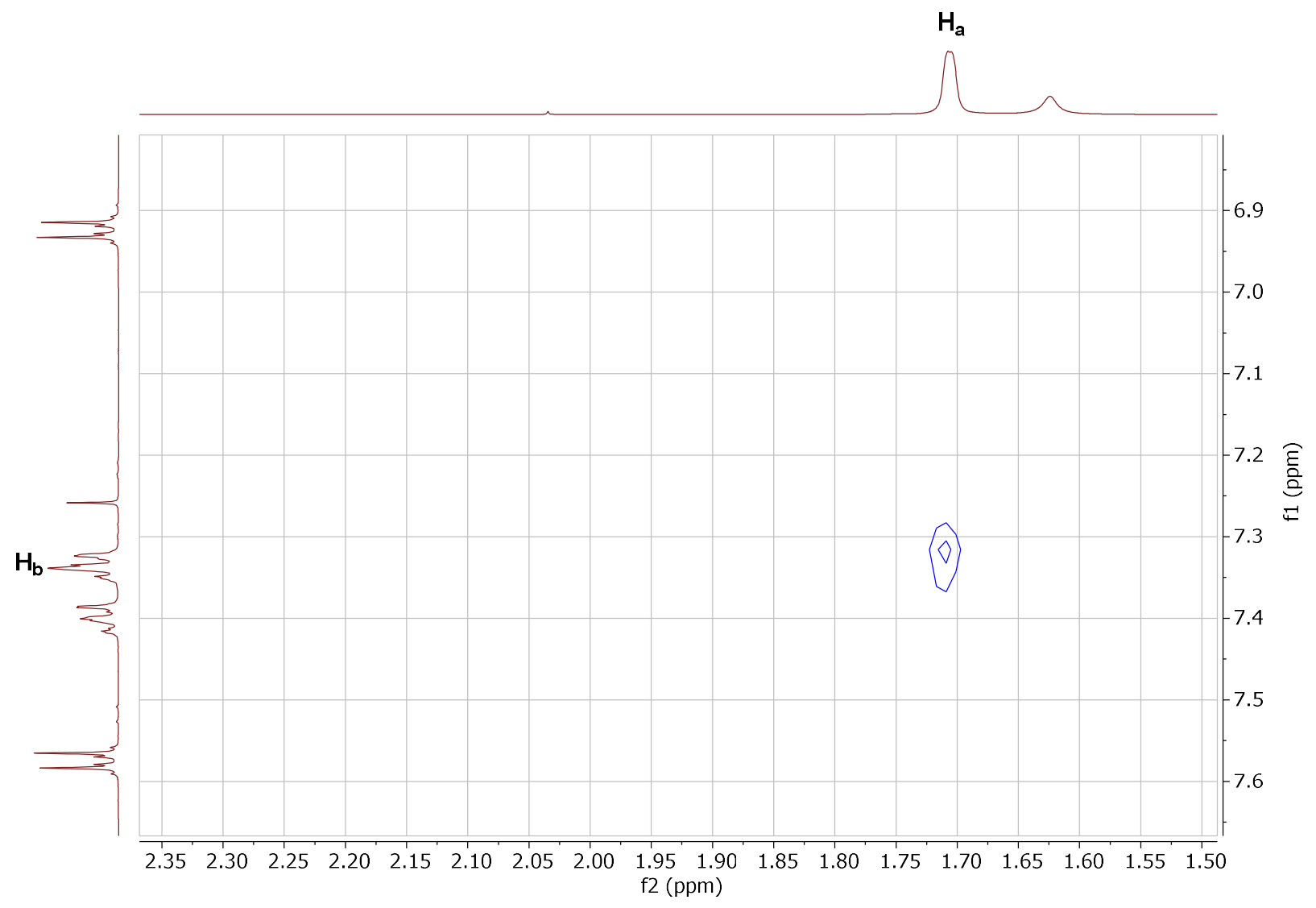


NOESY Spectra for Product $5 b$

NOESY Correlation: $\mathbf{5 b}$ (major diastereomer, $500 \mathrm{MHz}, \mathrm{CDCl}_{3}$ )

$\mathrm{MeO}$
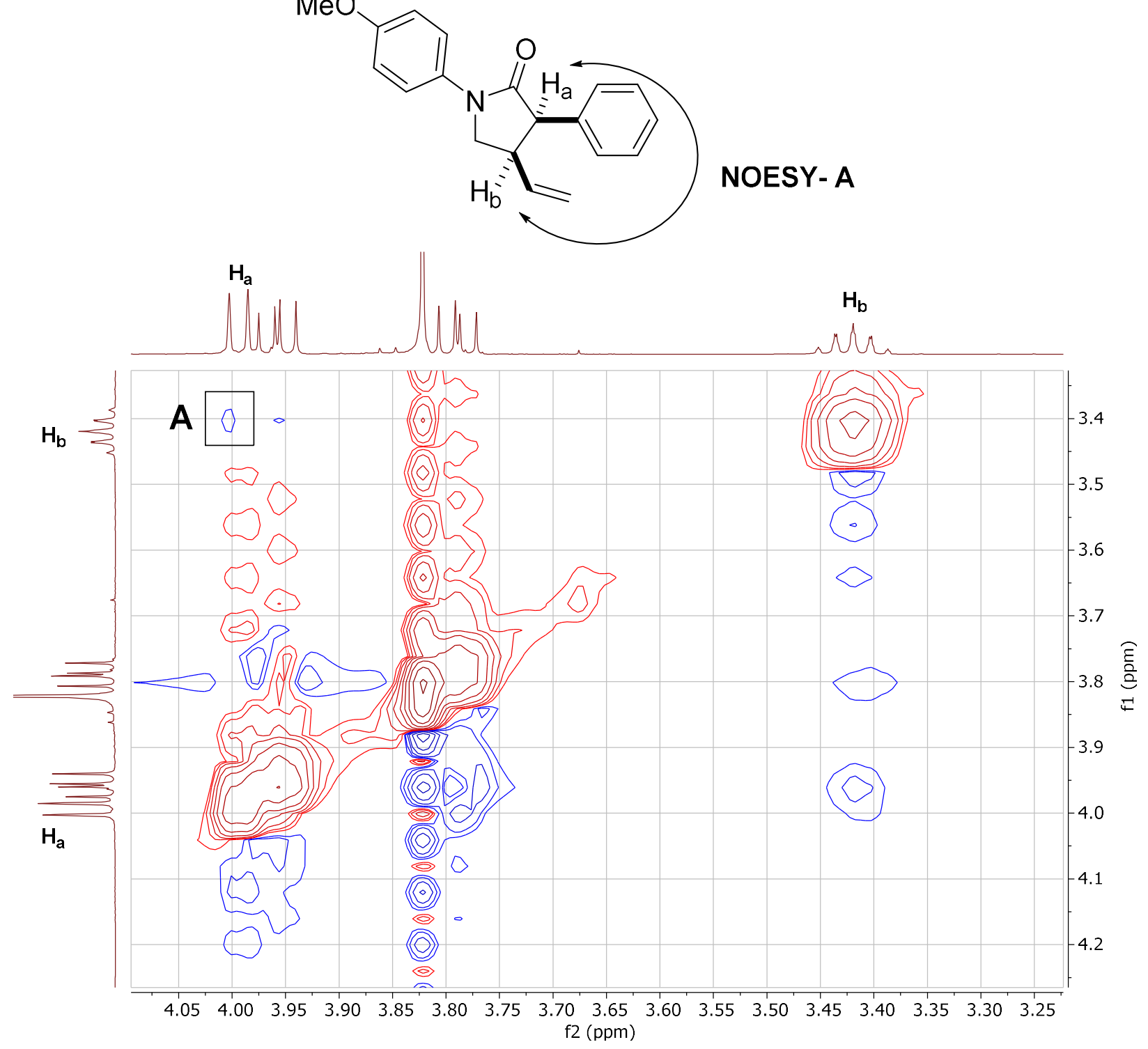
NOESY Spectra for Product 5c

NOESY Correlation: 5c (major diastereomer, $500 \mathrm{MHz}, \mathrm{CDCl}_{3}$ )

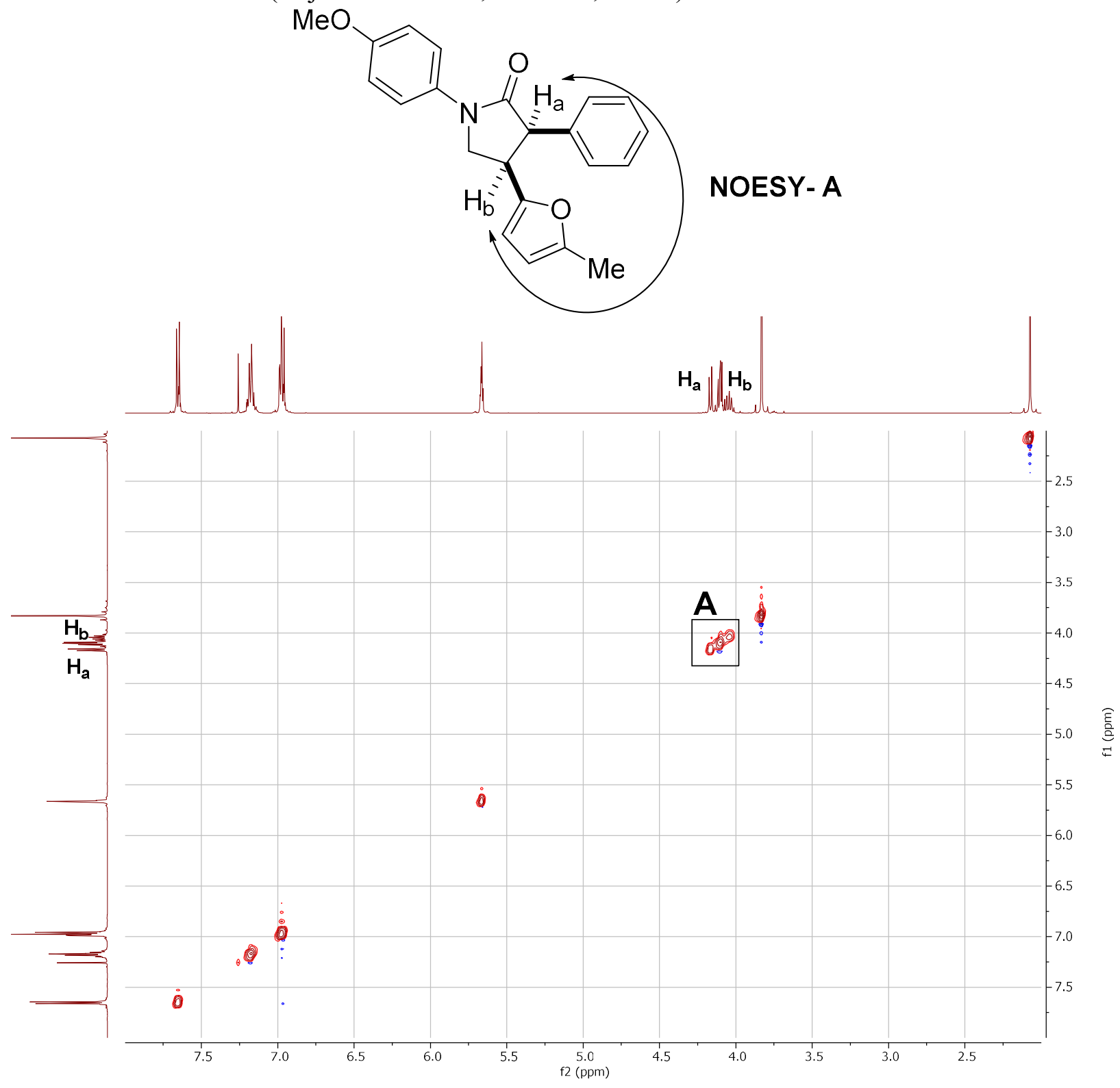




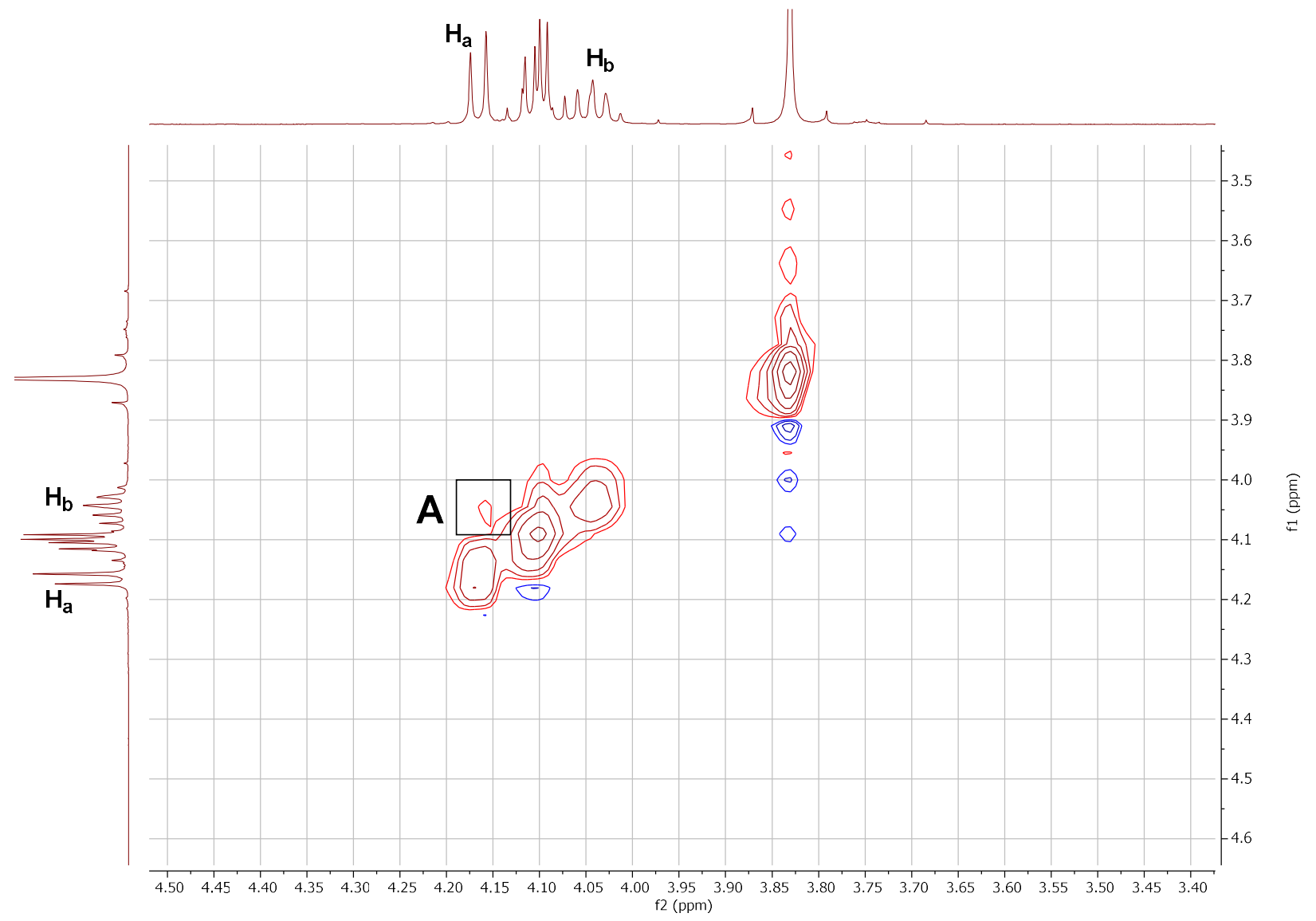


NMR Specta<smiles>COc1ccc(N(C/C=C/c2ccccc2)C(=O)Cl)cc1</smiles>

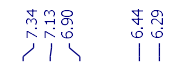

₹

$1 a$

$500 \mathrm{MHz}, \mathrm{CDCl}_{3}$
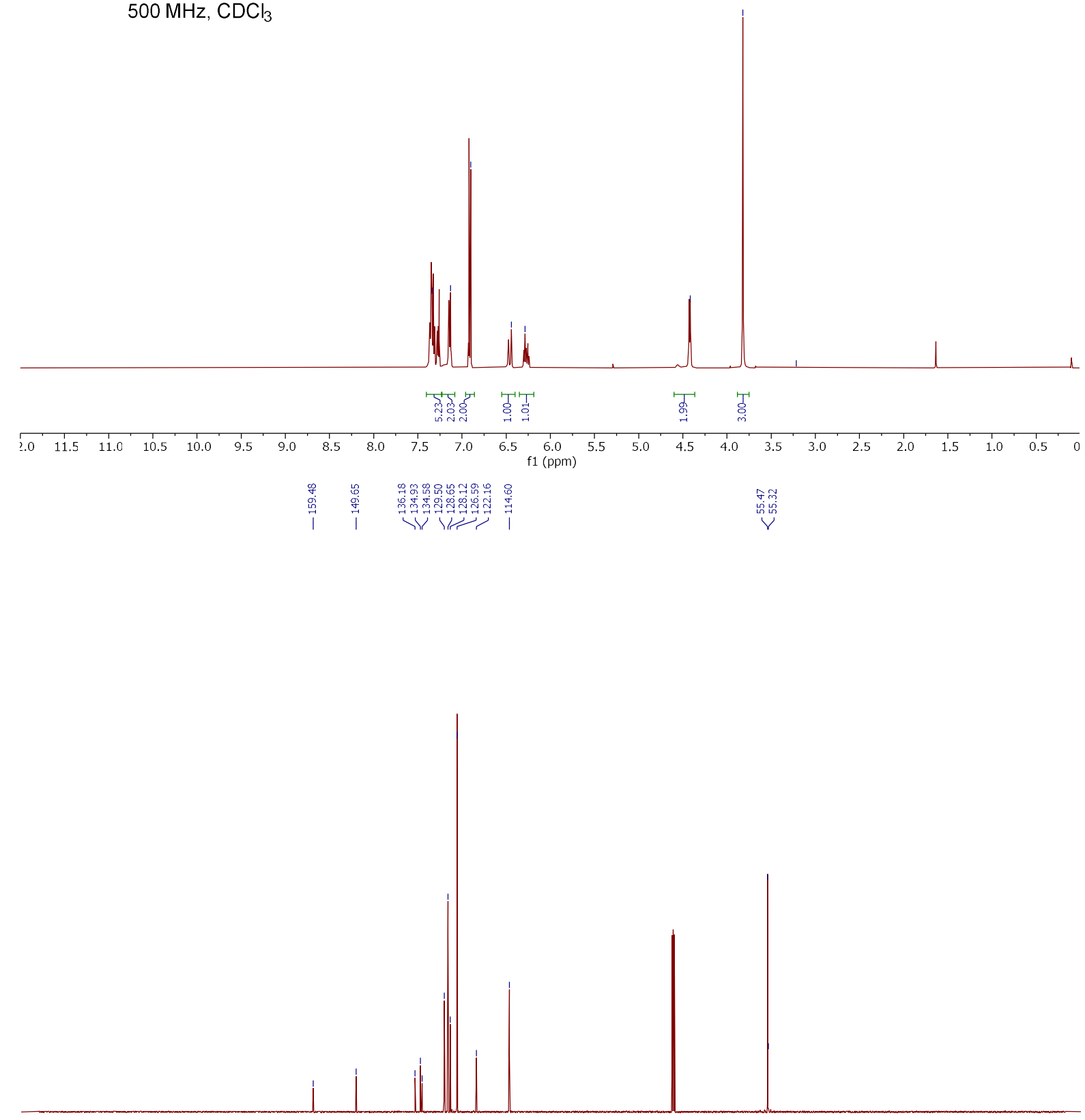

\begin{tabular}{llllllllllllllllllllllll}
1 & 1 \\
220 & 210 & 200 & 190 & 180 & 170 & 160 & 150 & 140 & 130 & 120 & $\begin{array}{c}1 \\
\mathrm{f} 1(\mathrm{ppm})\end{array}$ & 100 & 90 & 80 & 70 & 60 & 50 & 40 & 30 & 20 & 10 & 0 & -10 \\
\hline
\end{tabular} 

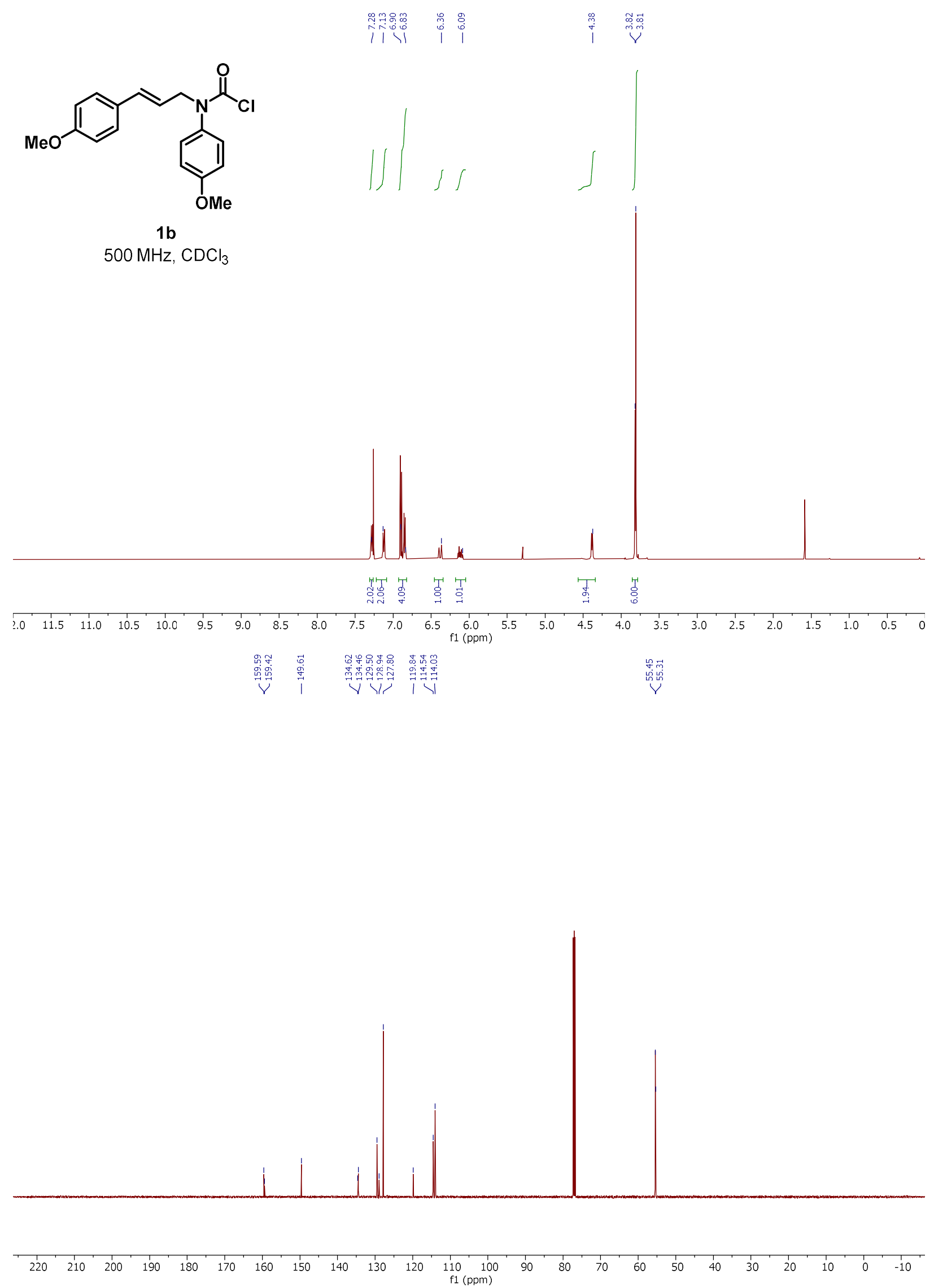

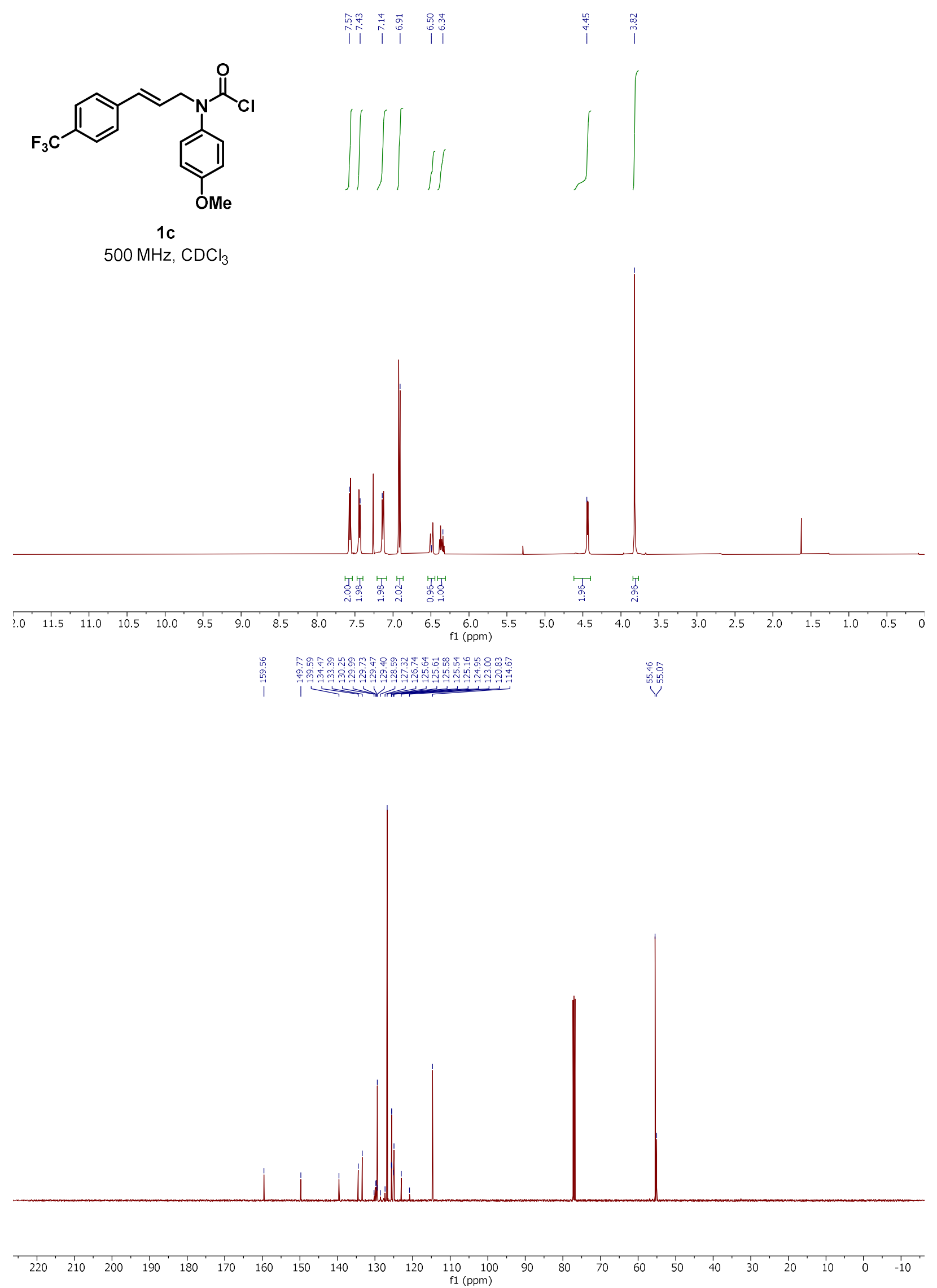
$400 \mathrm{MHz}: \mathrm{CDCl}_{3}$

\begin{tabular}{|c|c|c|c|c|c|c|c|c|c|c|c|c|c|c|c|c|c|c|c|}
\hline 170 & 150 & 130 & 110 & 90 & 70 & 50 & 30 & 10 & $\begin{array}{l}-10 \\
\mathrm{f} 1(\mathrm{ppm})\end{array}$ & -30 & -50 & -70 & -90 & -110 & -130 & -150 & -170 & -190 & -211 \\
\hline
\end{tabular}


<smiles>COc1ccc(N(C/C=C/c2ccc(F)cc2)C(=O)Cl)cc1</smiles>

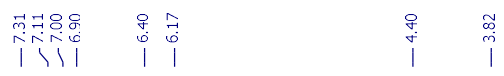

1d

$500 \mathrm{MHz}, \mathrm{CDCl}_{3}$
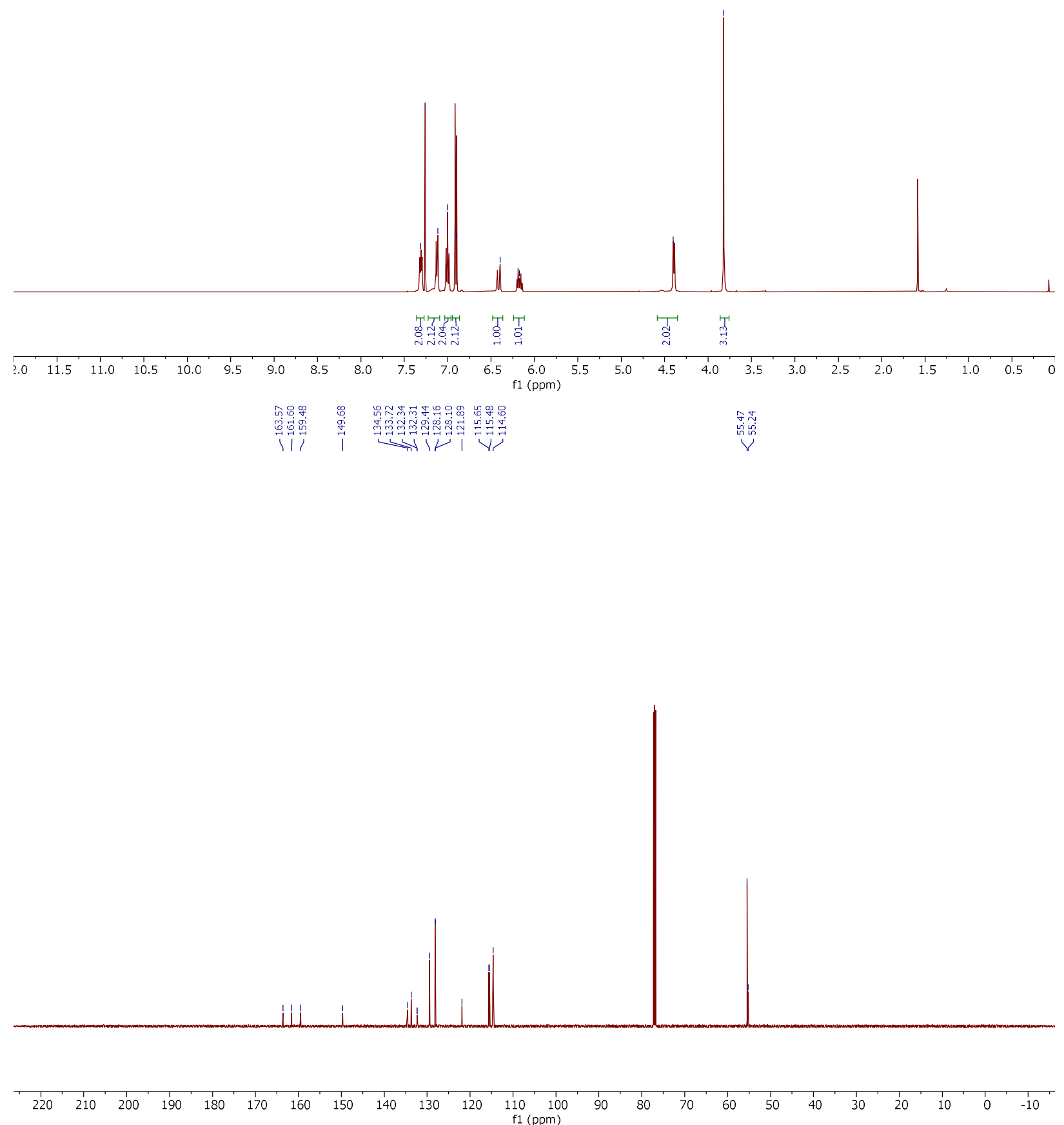
$400 \mathrm{MHz}, \mathrm{CDCl}_{3}$

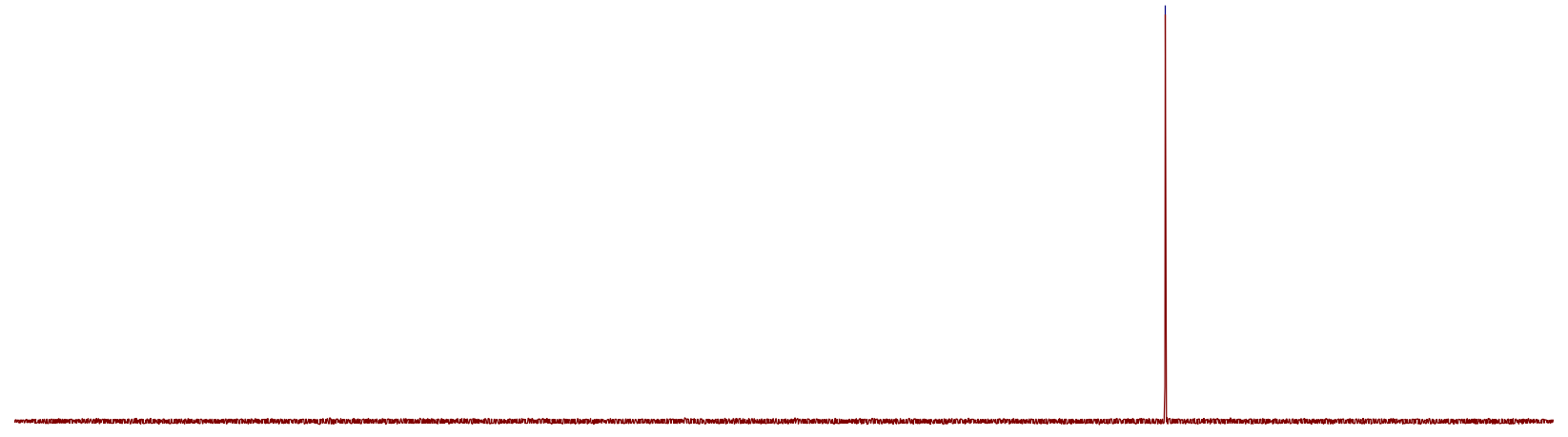

$\begin{array}{lllllllllllllllllllll}170 & 150 & 130 & 110 & 90 & 70 & 50 & 30 & 10 & -10 & -30 & -50 & -70 & -90 & -110 & -130 & -150 & -170 & -190 & -211\end{array}$ 

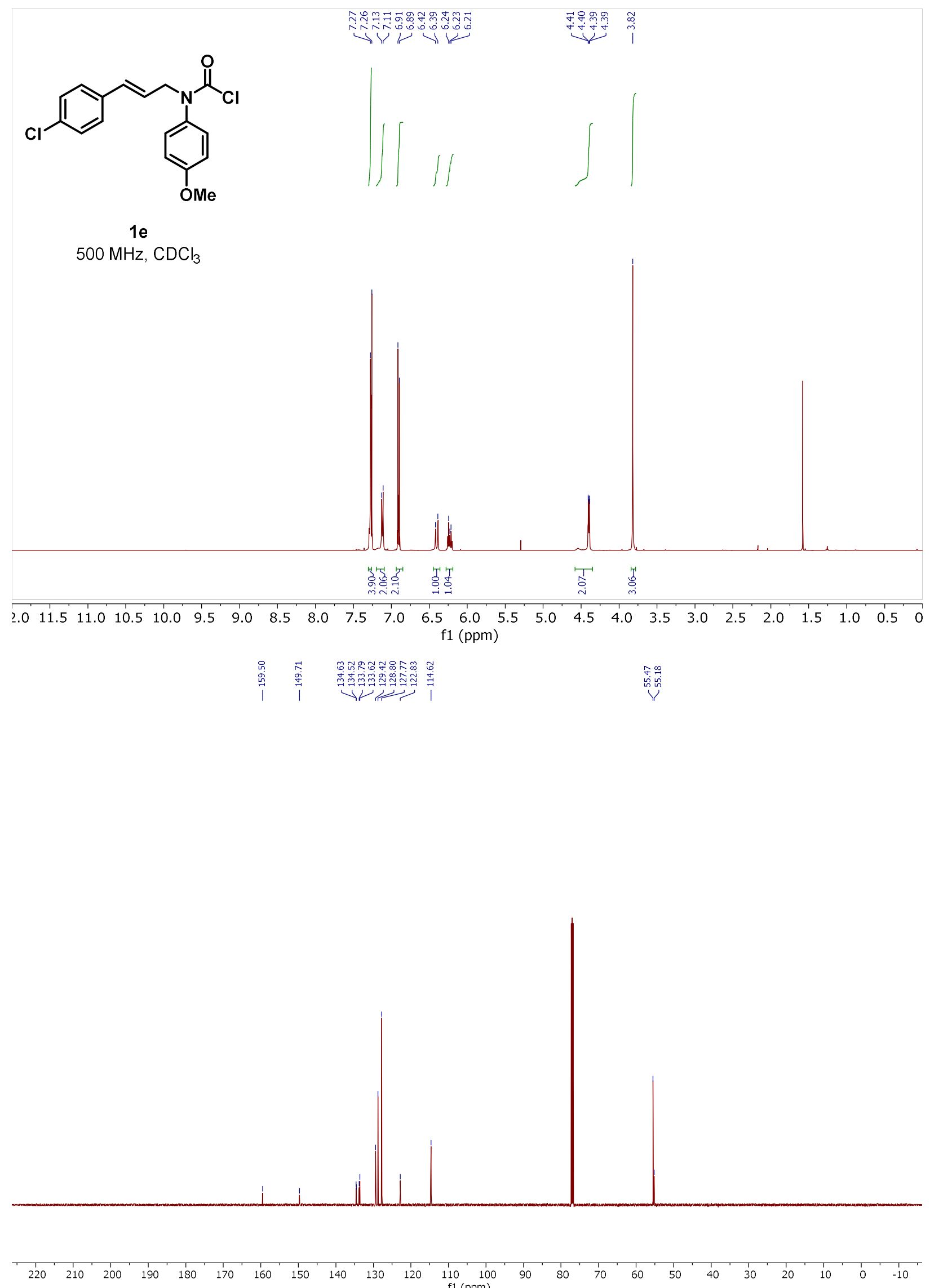
<smiles>COc1ccc(N(C/C=C/c2ccc(Br)cc2)C(=O)Cl)cc1</smiles>

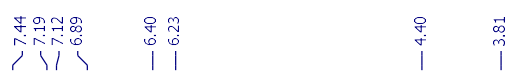

$1 f$

$500 \mathrm{MHz}, \mathrm{CDCl}_{3}$
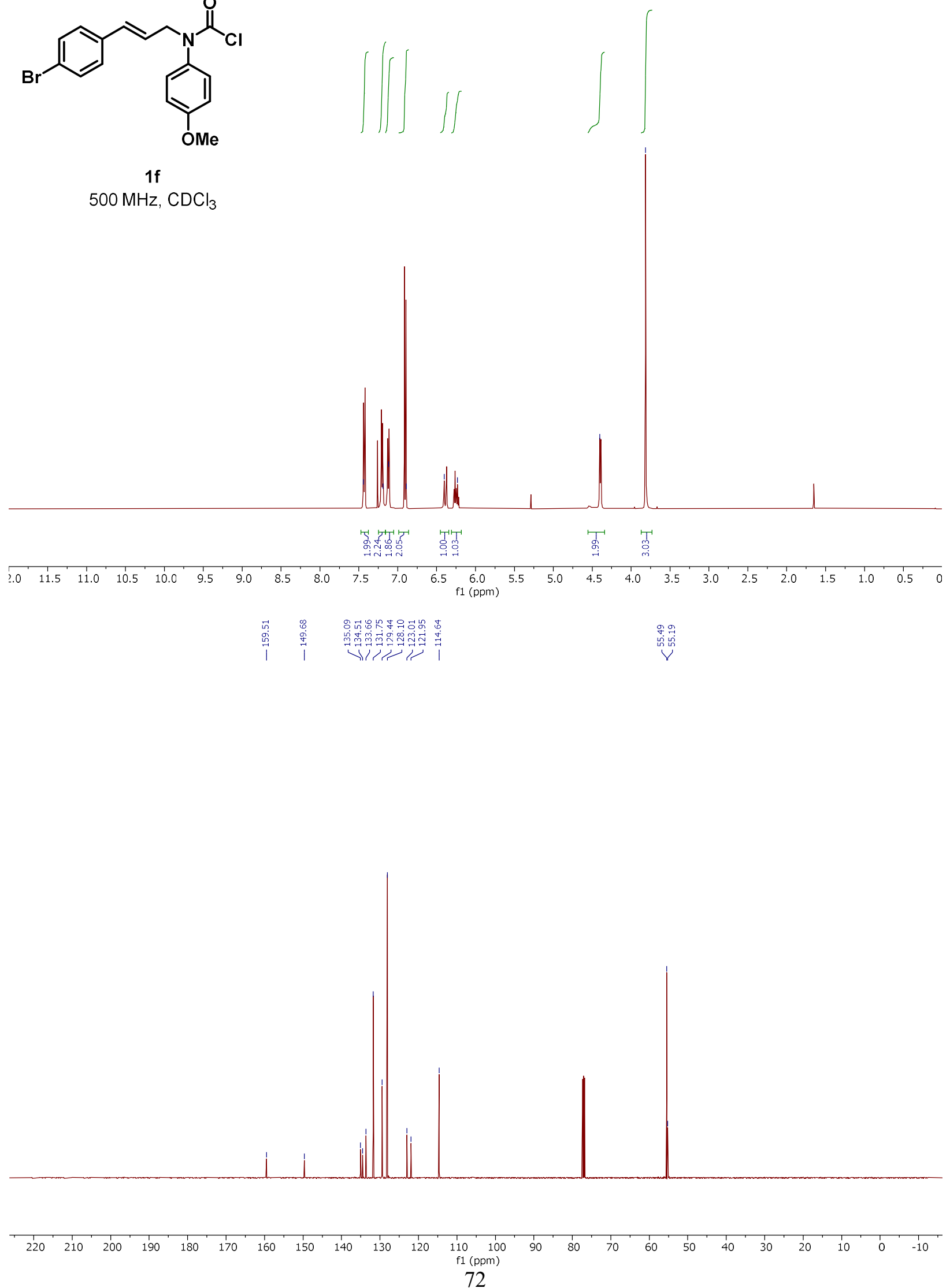

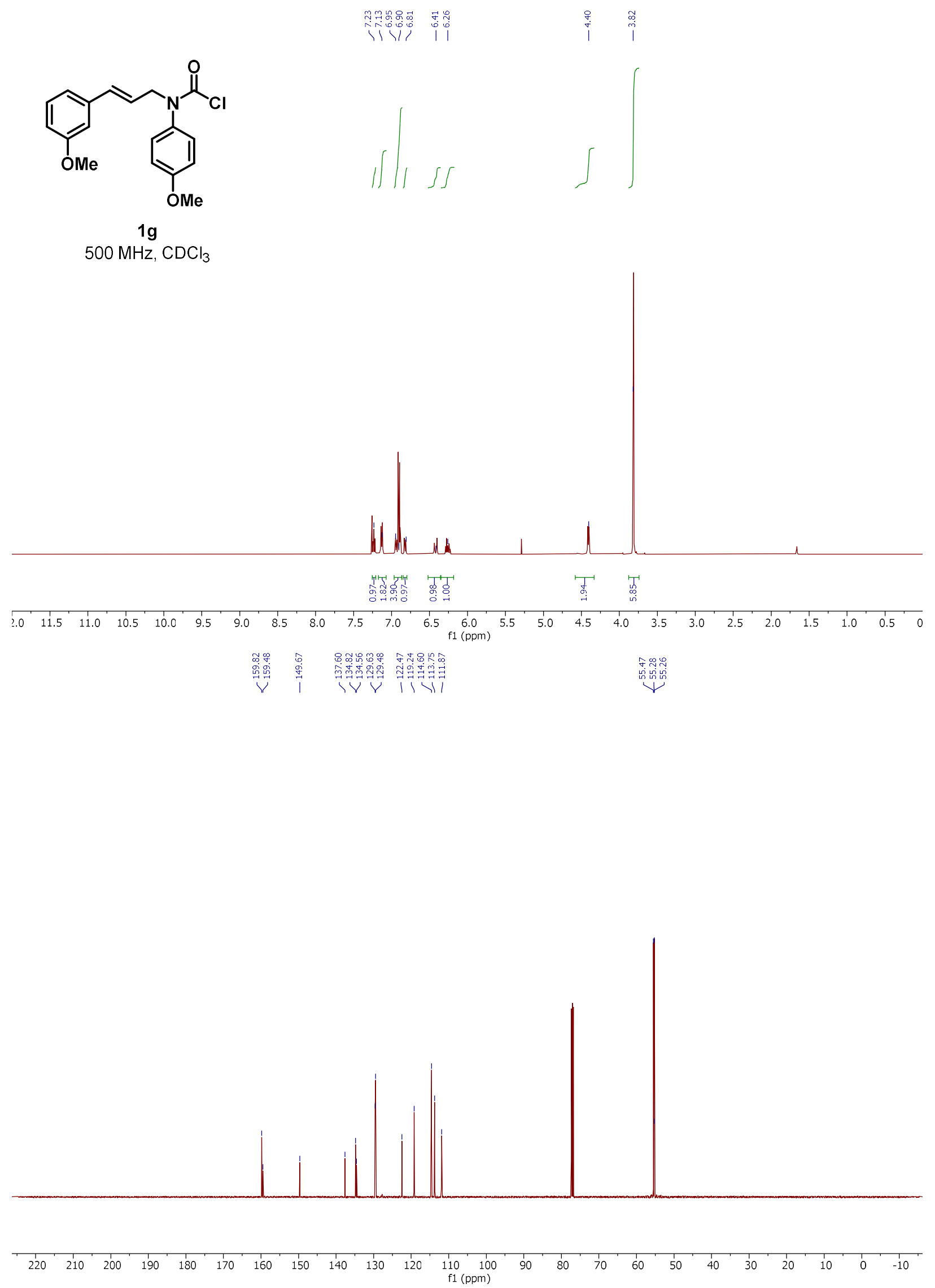

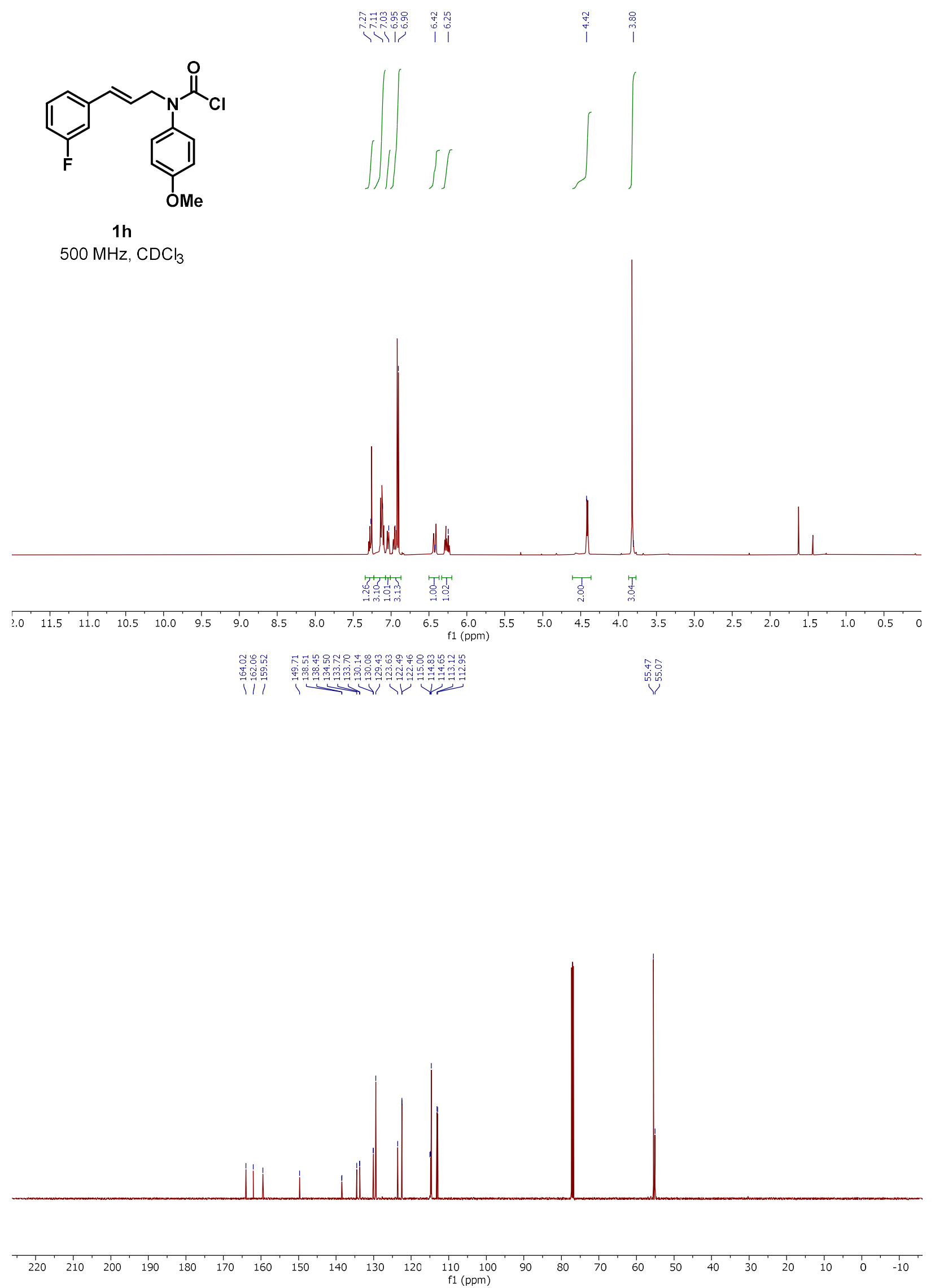
$400 \mathrm{MHz}, \mathrm{CDCl}_{3}$

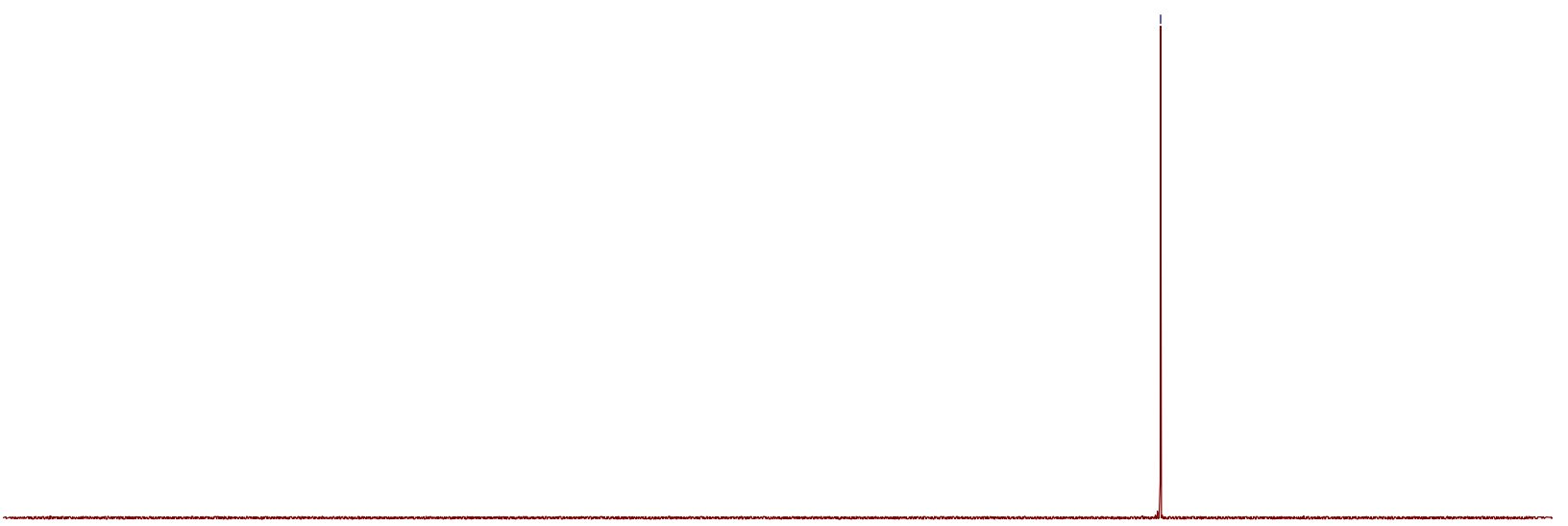

$\begin{array}{lllllllllllllllllllllll}170 & 150 & 130 & 110 & 90 & 70 & 50 & 30 & 10 & -10 & -30 & -50 & -10 & -90 & -110 & -130 & -150 & -170 & -190 & -211\end{array}$


<smiles>COc1ccc(N(C/C=C/c2cccc(Cl)c2)C(=O)Cl)cc1</smiles>

$1 \mathrm{i}$

$500 \mathrm{MHz}, \mathrm{CDCl}_{3}$
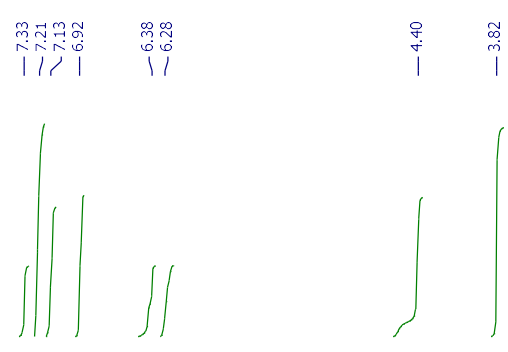

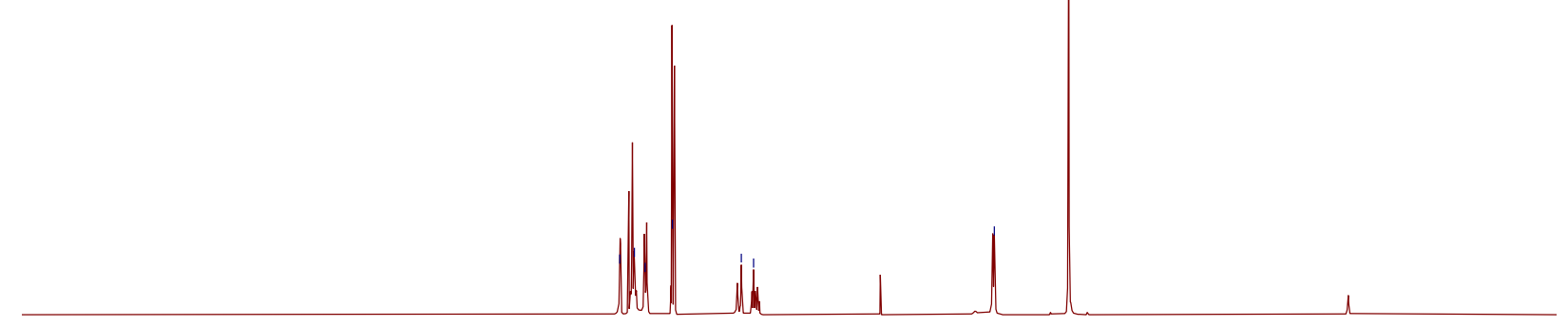

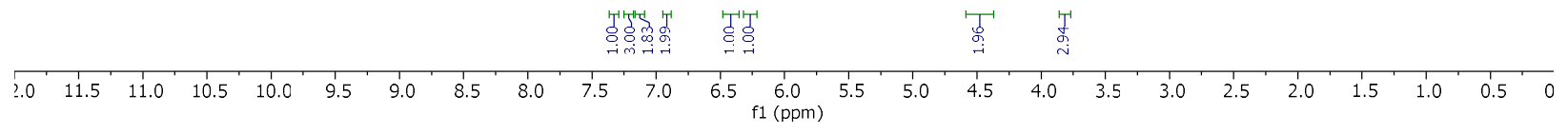

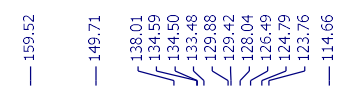
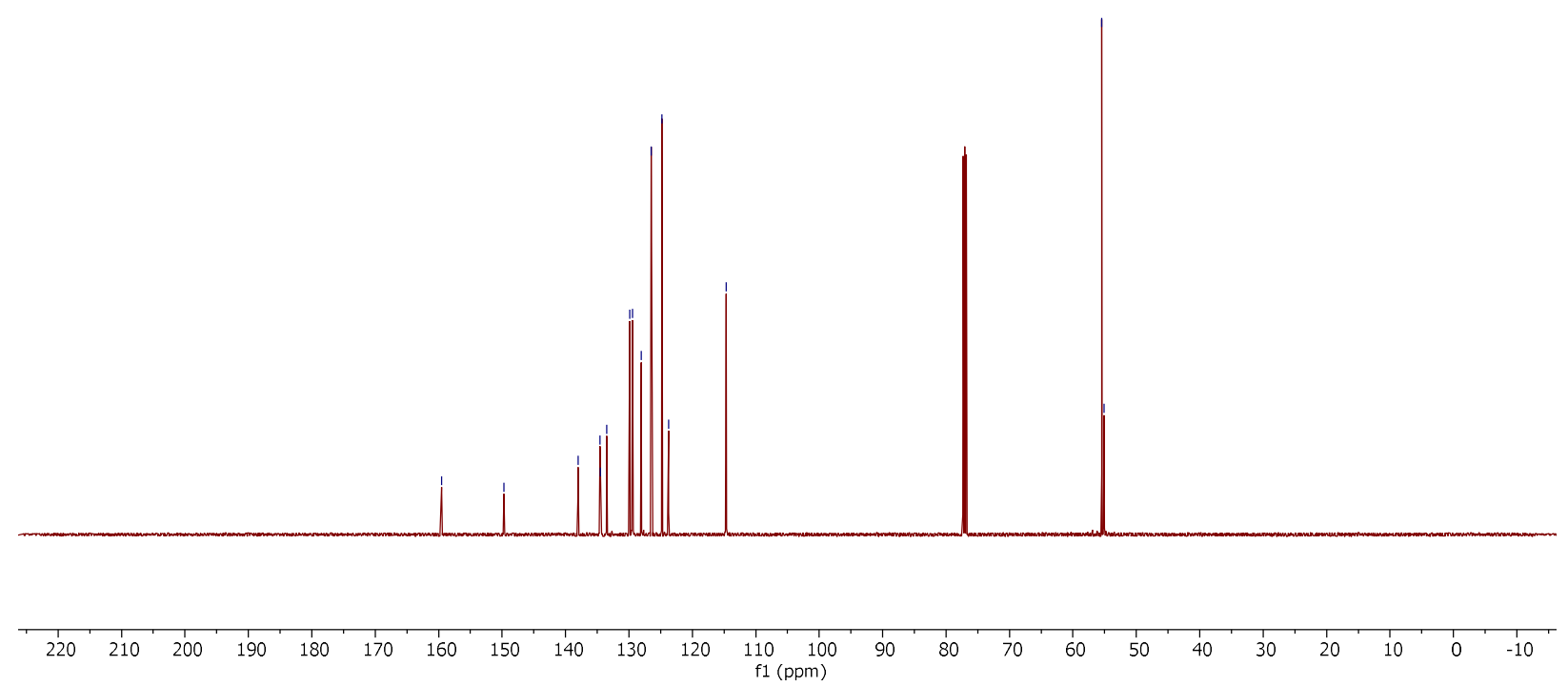


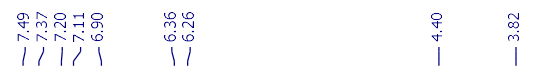<smiles>COc1ccc(N(C/C=C/c2cccc(Br)c2)C(=O)Cl)cc1</smiles>

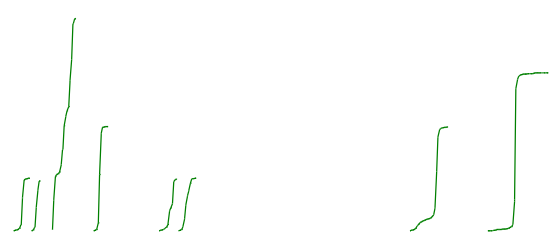

1j

$500 \mathrm{MHz}, \mathrm{CDCl}_{3}$
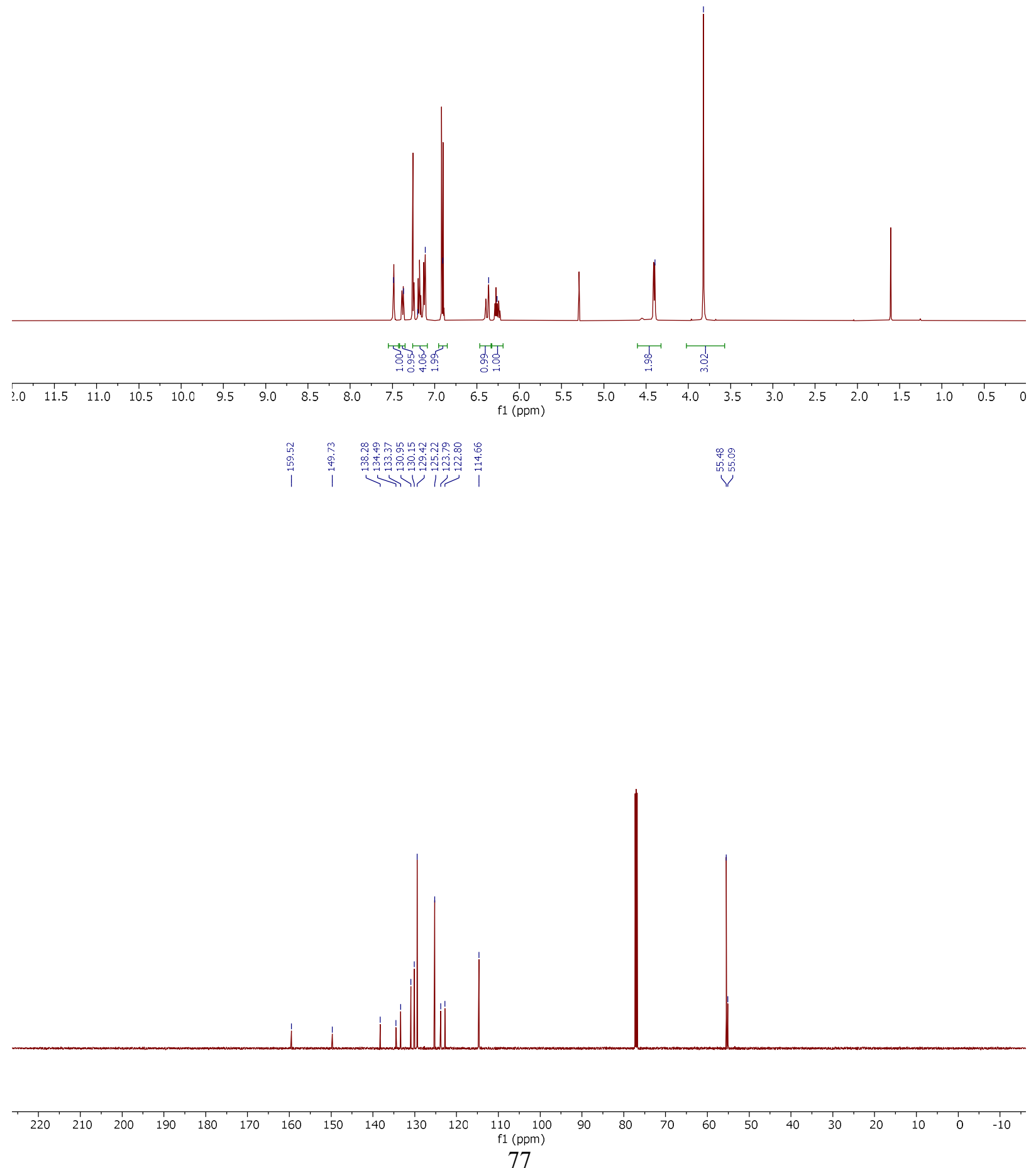


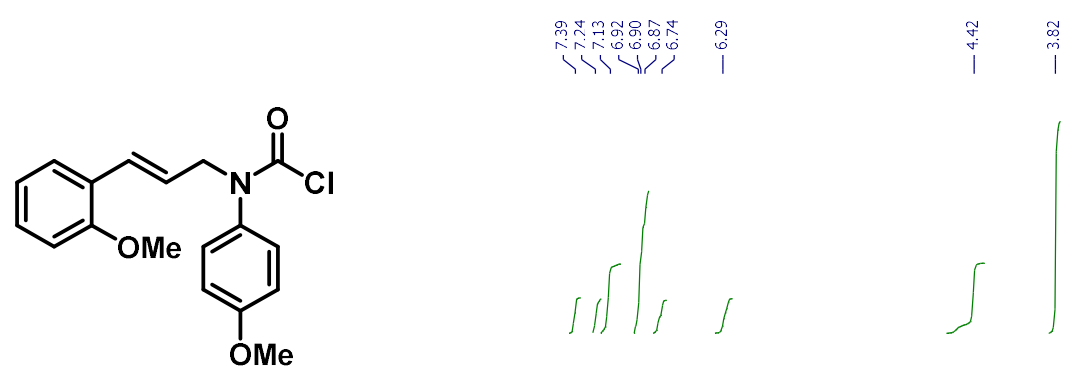

$1 \mathrm{k}$

$500 \mathrm{MHz} \mathrm{CDCl}_{3}$
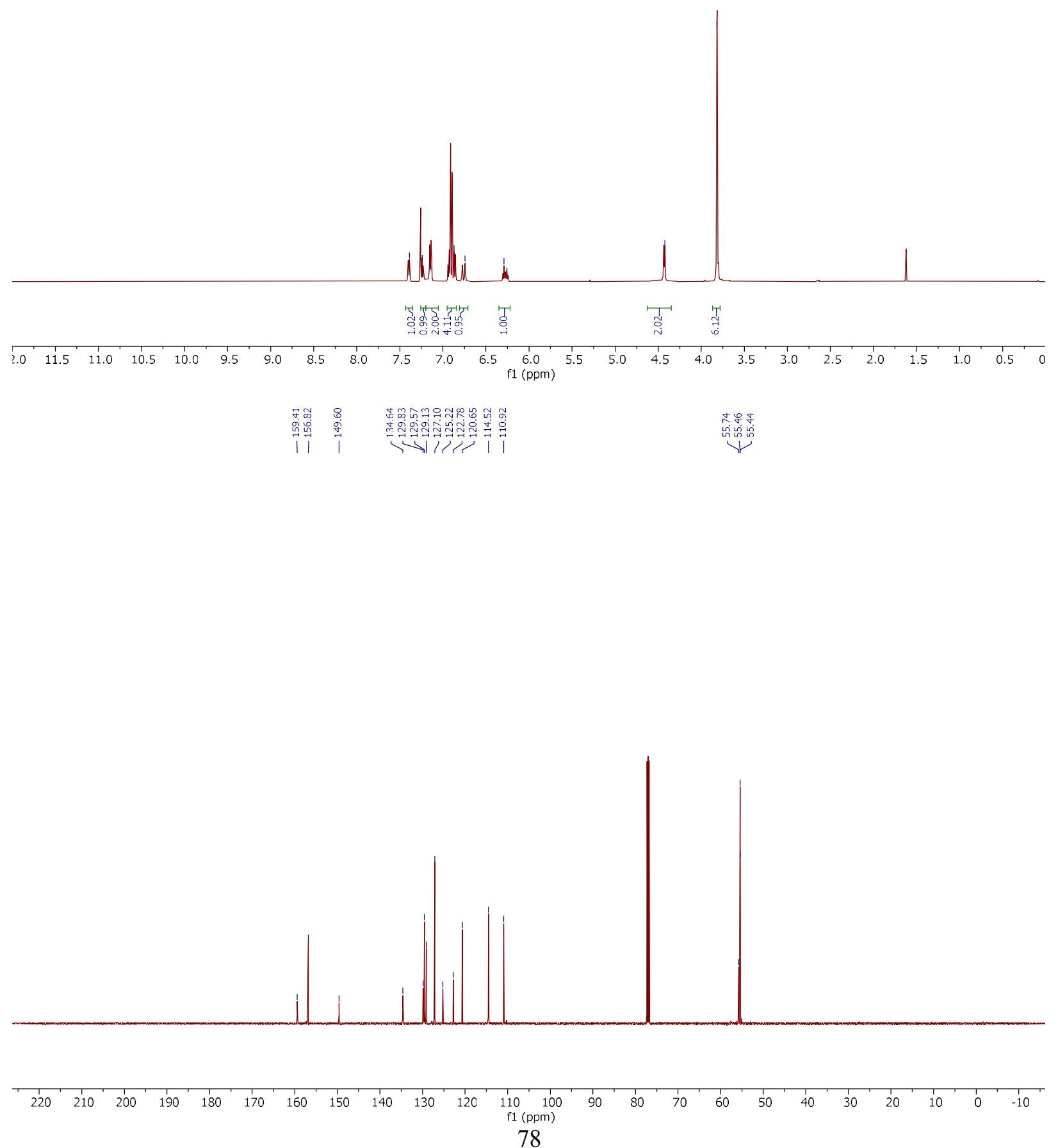

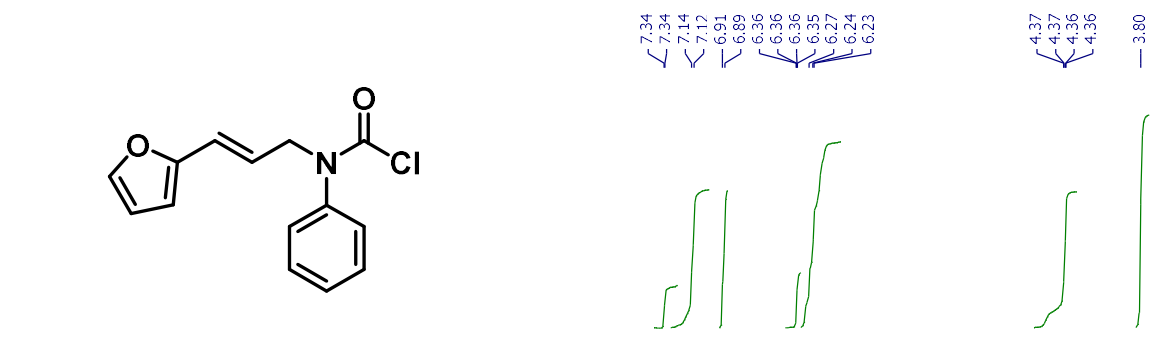

11

$500 \mathrm{MHz}, \mathrm{CDCl}_{3}$
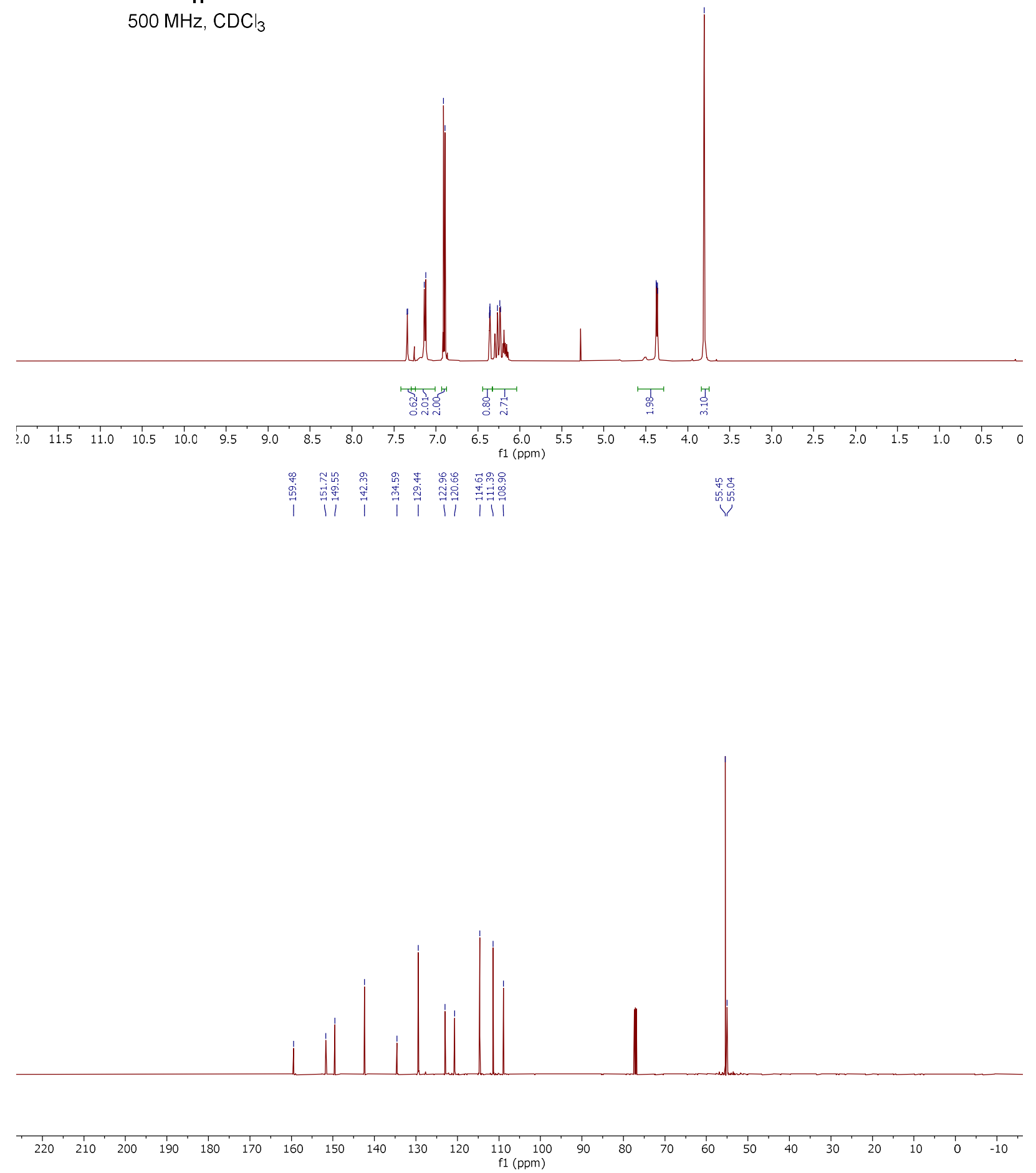

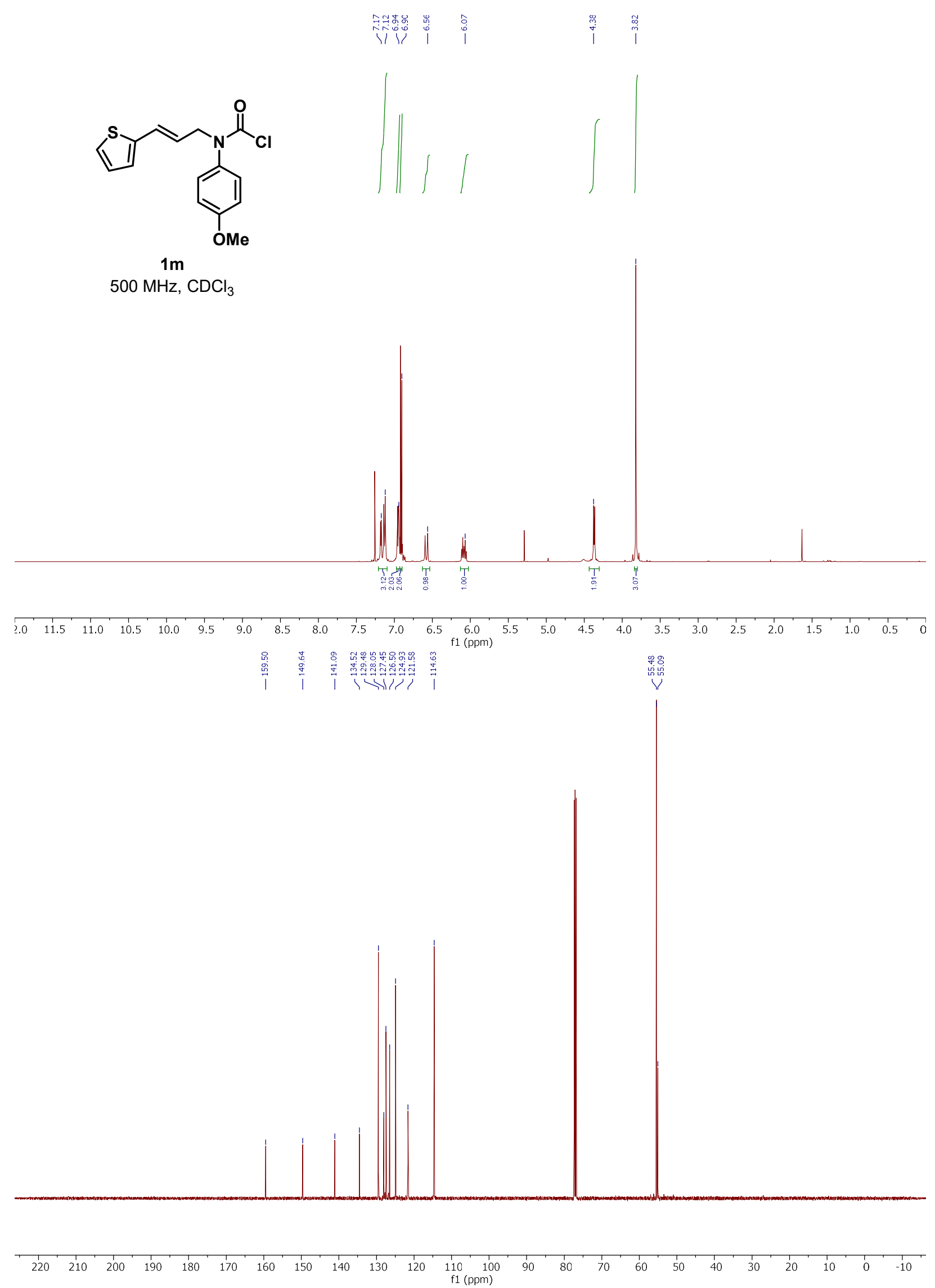

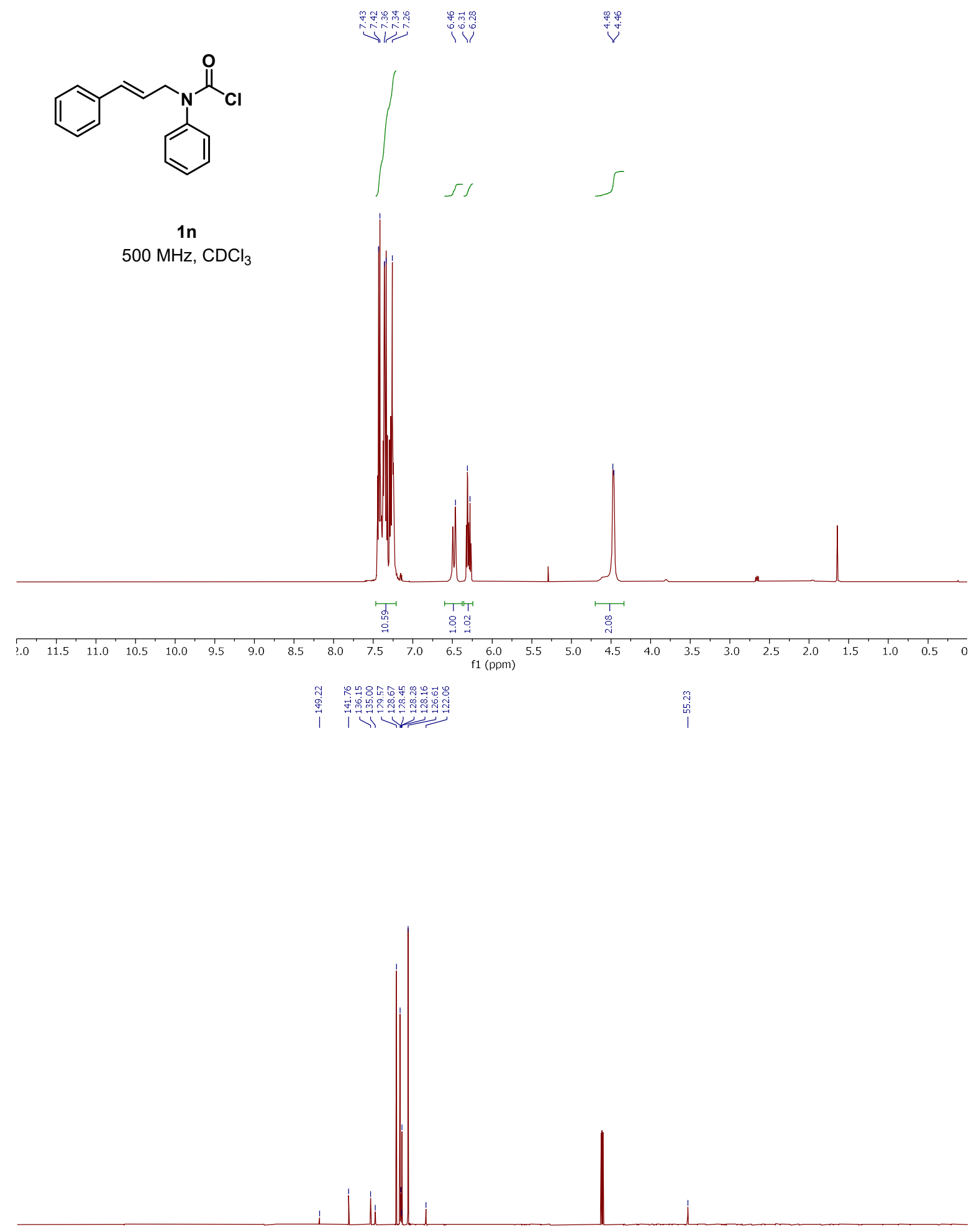

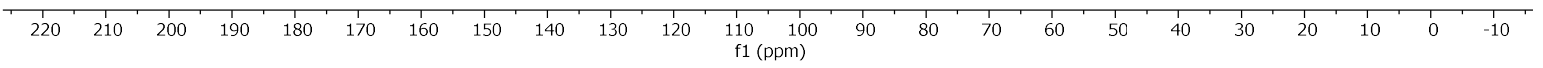




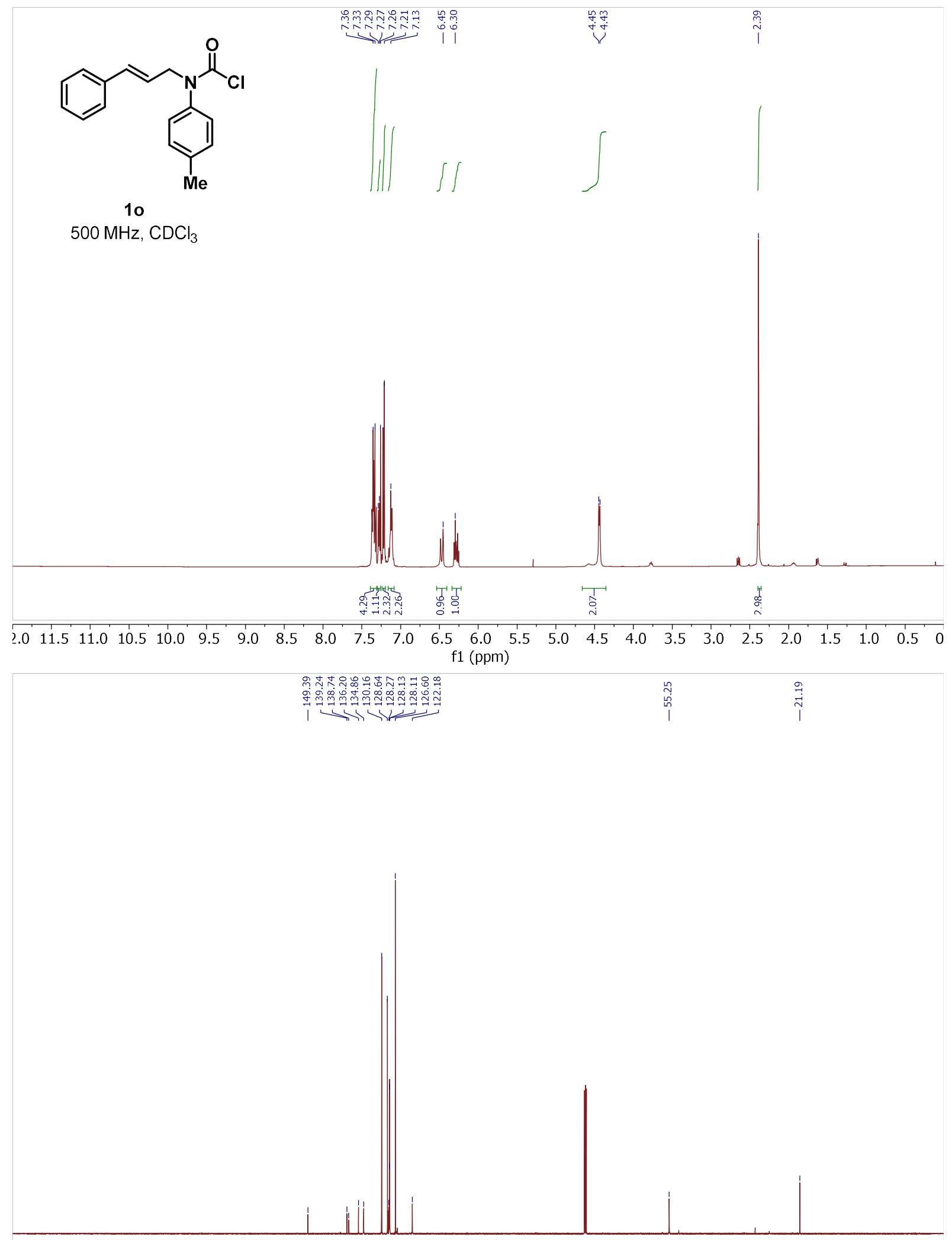

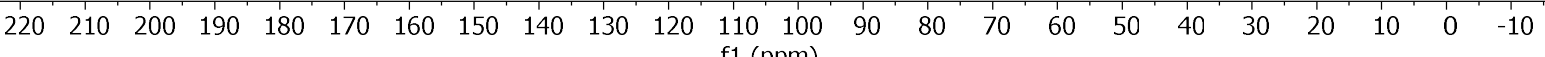


<smiles>COc1ccc(CN(C/C=C/c2ccccc2)C(=O)Cl)cc1</smiles>

$1 p$

$500 \mathrm{MHz}, \mathrm{CDCl}_{3}$

1.2:1 mixture of rotamers
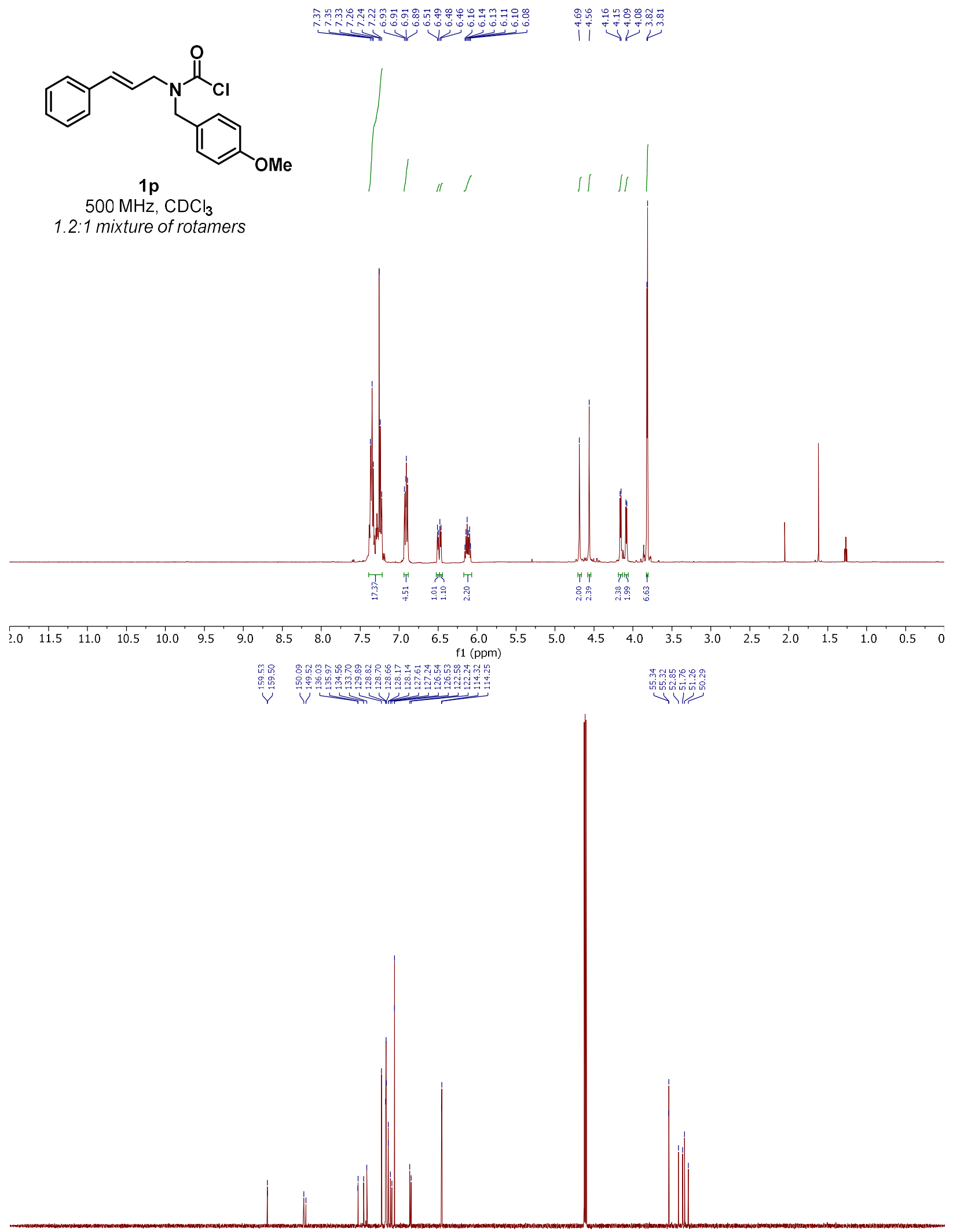

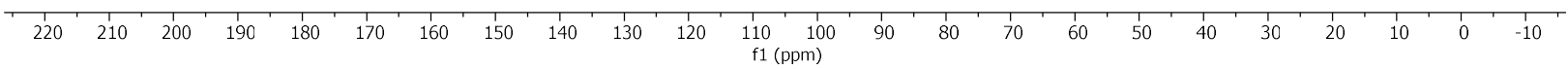



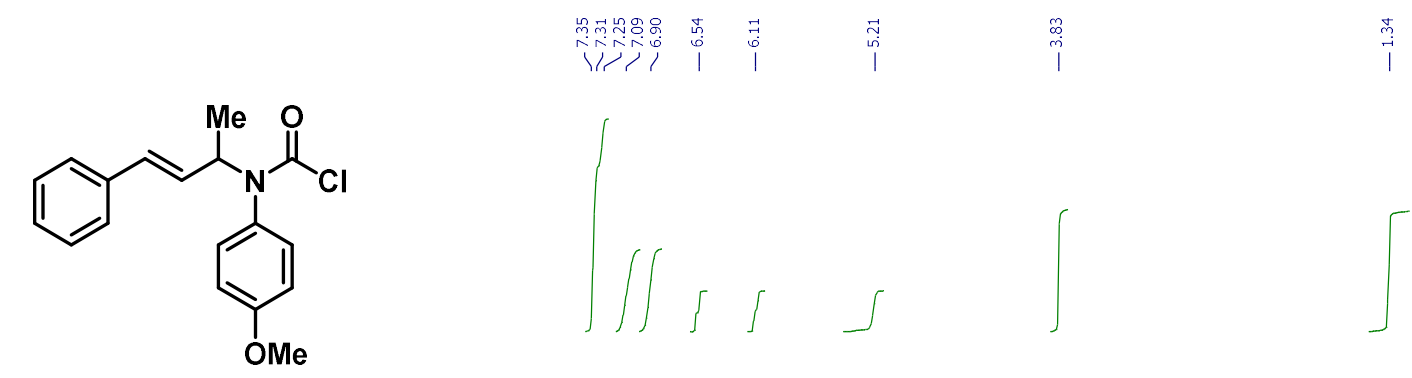

$1 q$

$500 \mathrm{MHz}^{\mathrm{CDCl}_{3}}$
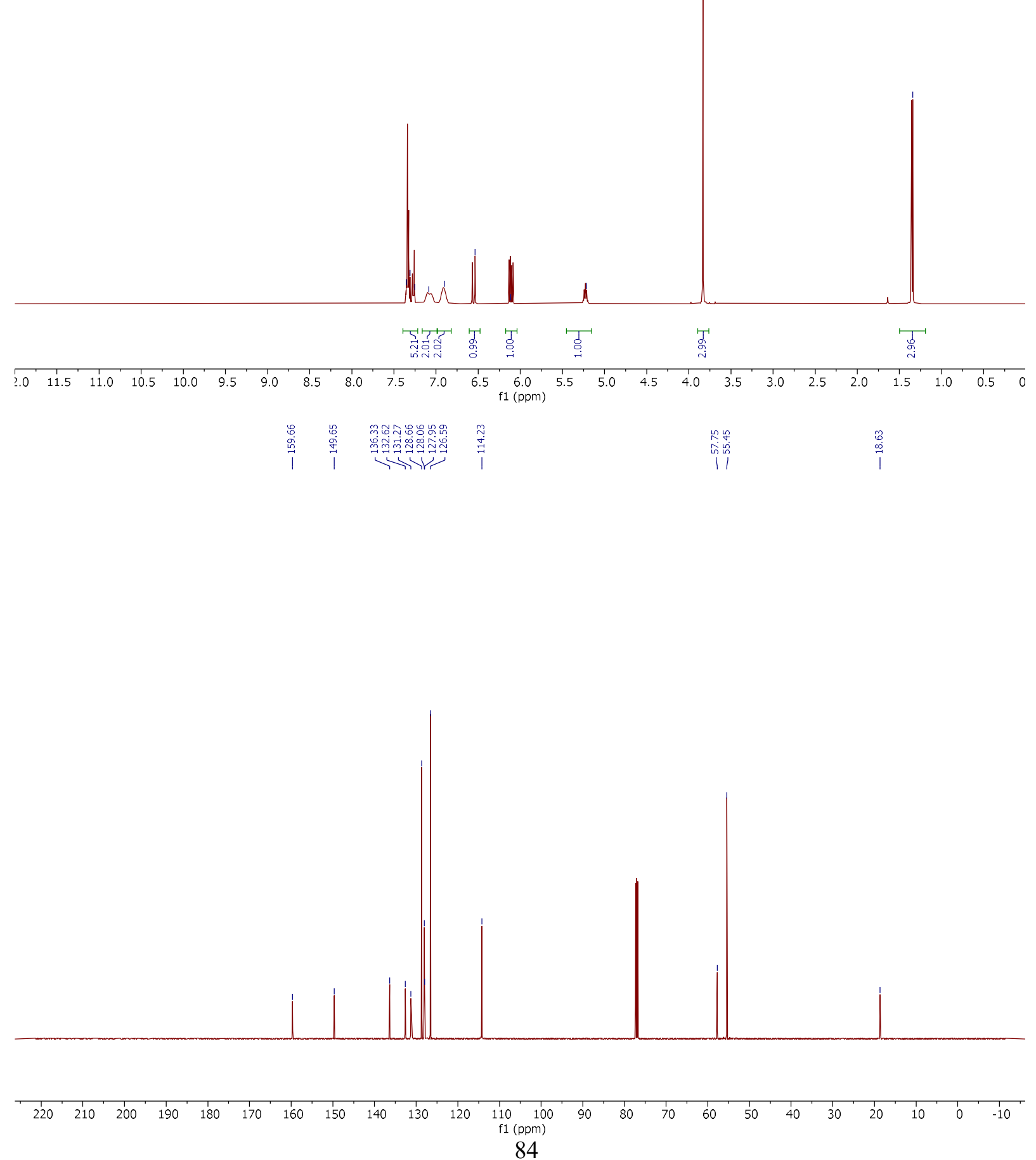


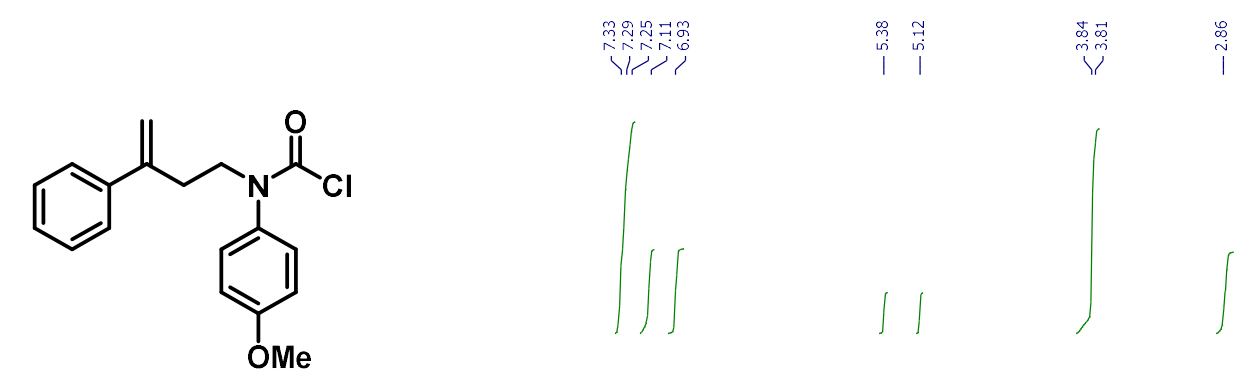

3a

$500 \mathrm{MHz}, \mathrm{CDCl}_{3}$
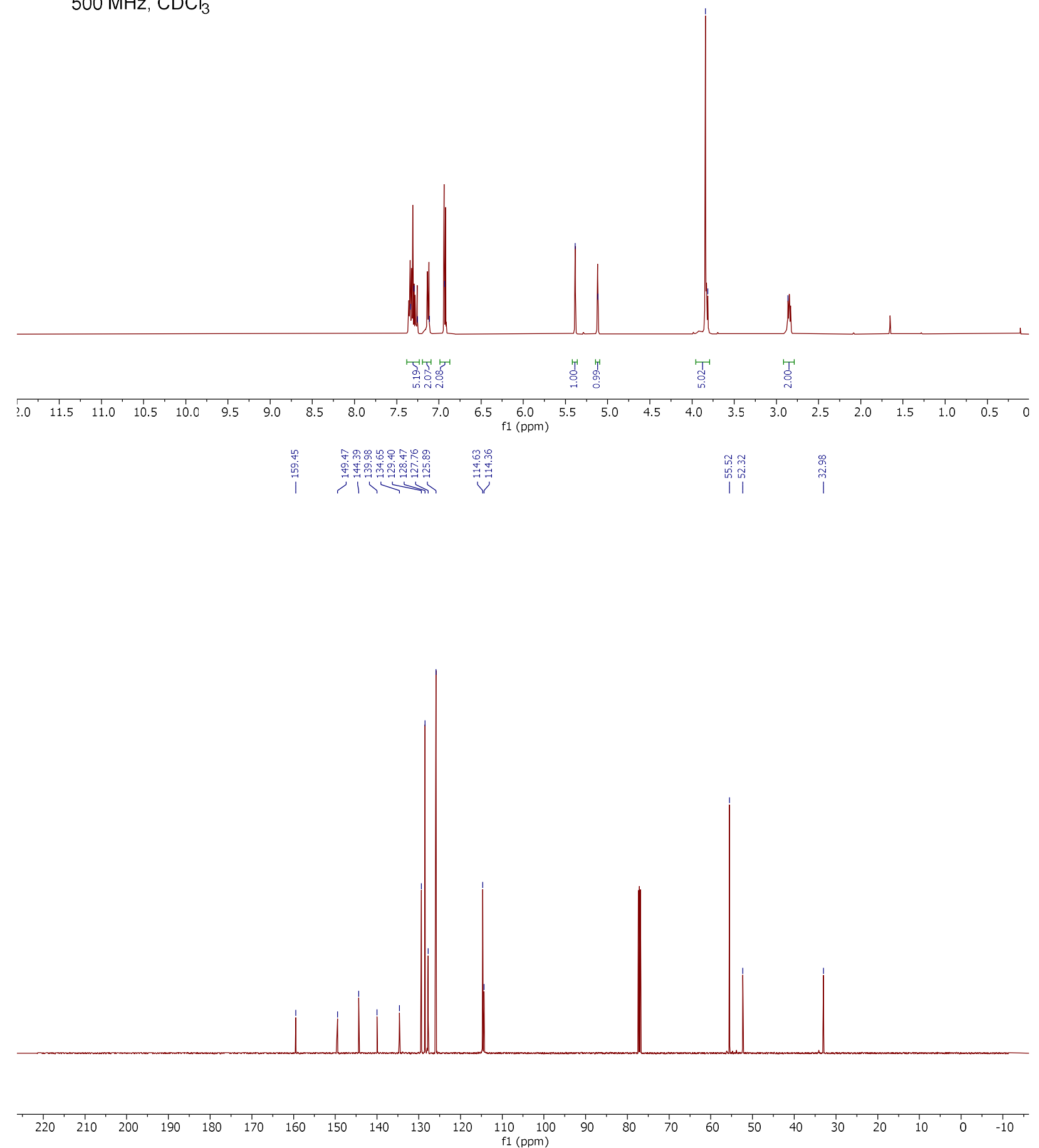
<smiles>C=C(CCN(C(=O)Cl)c1ccc(OC)cc1)c1ccccc1OC</smiles>

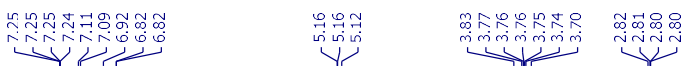

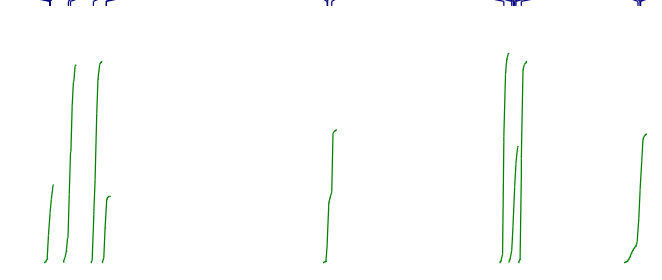

$3 b$

$500 \mathrm{MHz}, \mathrm{CDCl}_{3}$
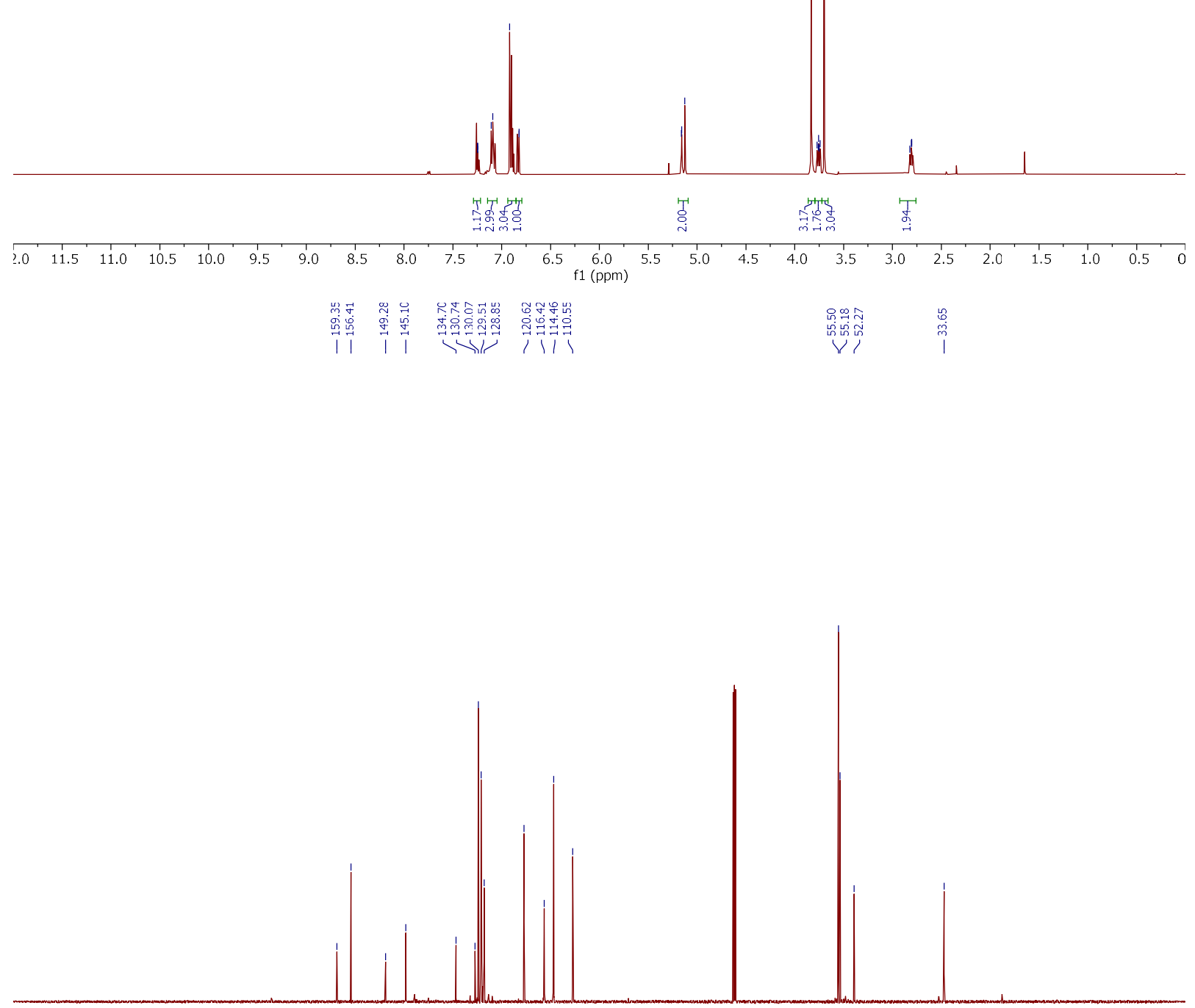

$\begin{array}{lllllllllllllllllllllllllll}1 & 1 \\ 220 & 210 & 200 & 190 & 180 & 170 & 160 & 150 & 140 & 130 & 120 & 110 & 100 & 90 & 80 & 70 & 60 & 50 & 40 & 30 & 20 & 10 & 0 & -10\end{array}$ 


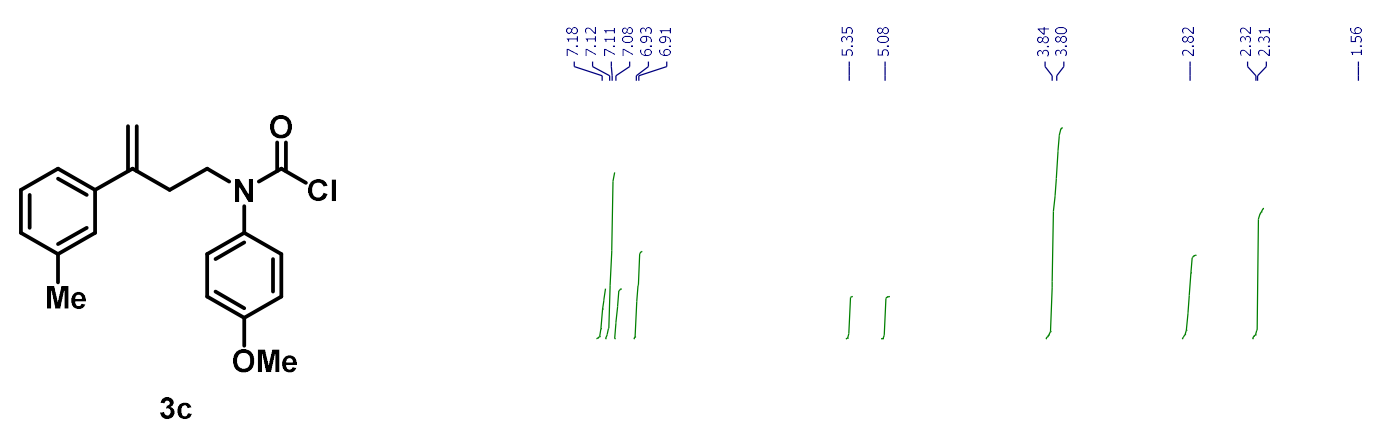

$500 \mathrm{MHz}, \mathrm{CDCl}_{3}$
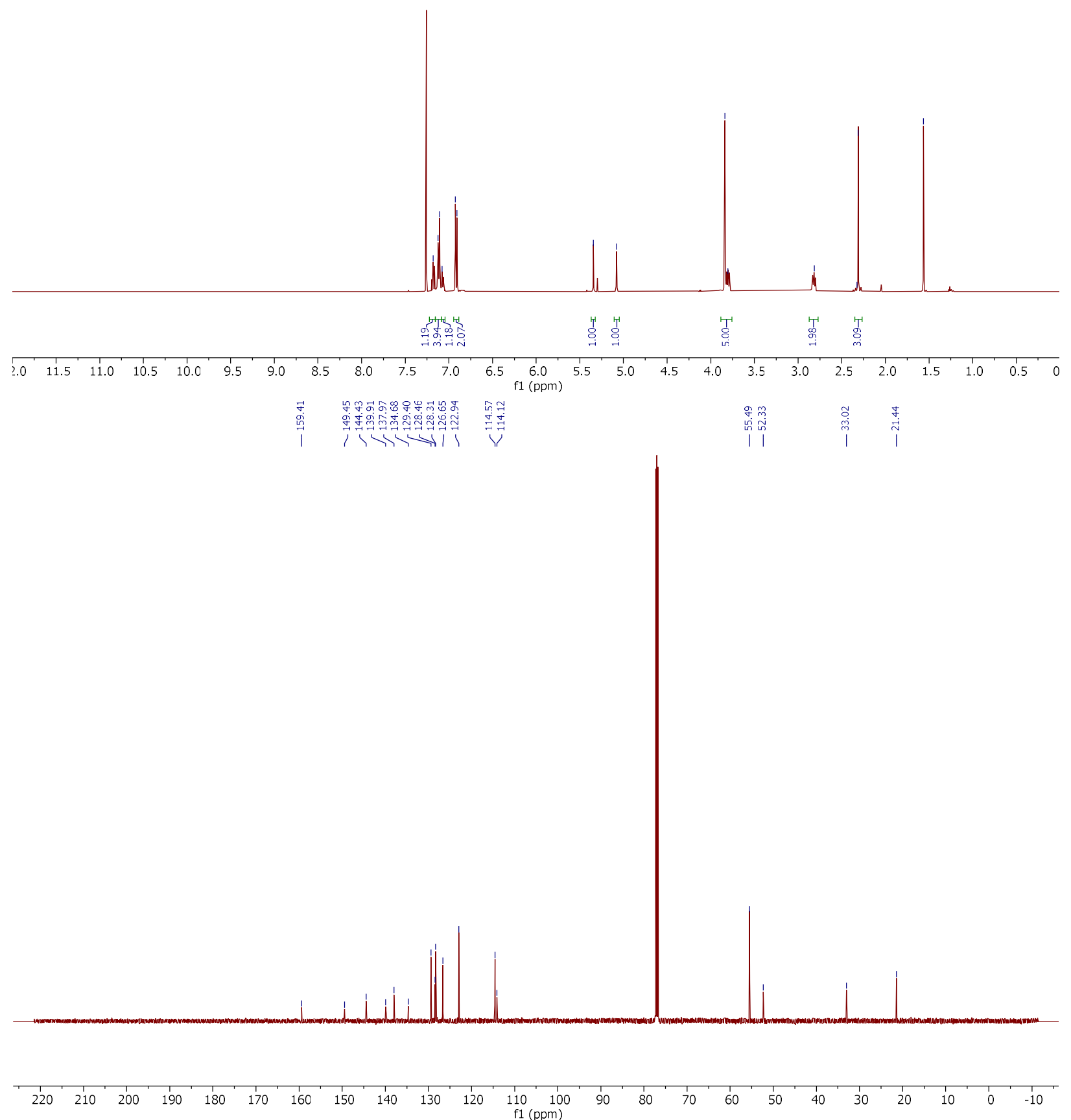

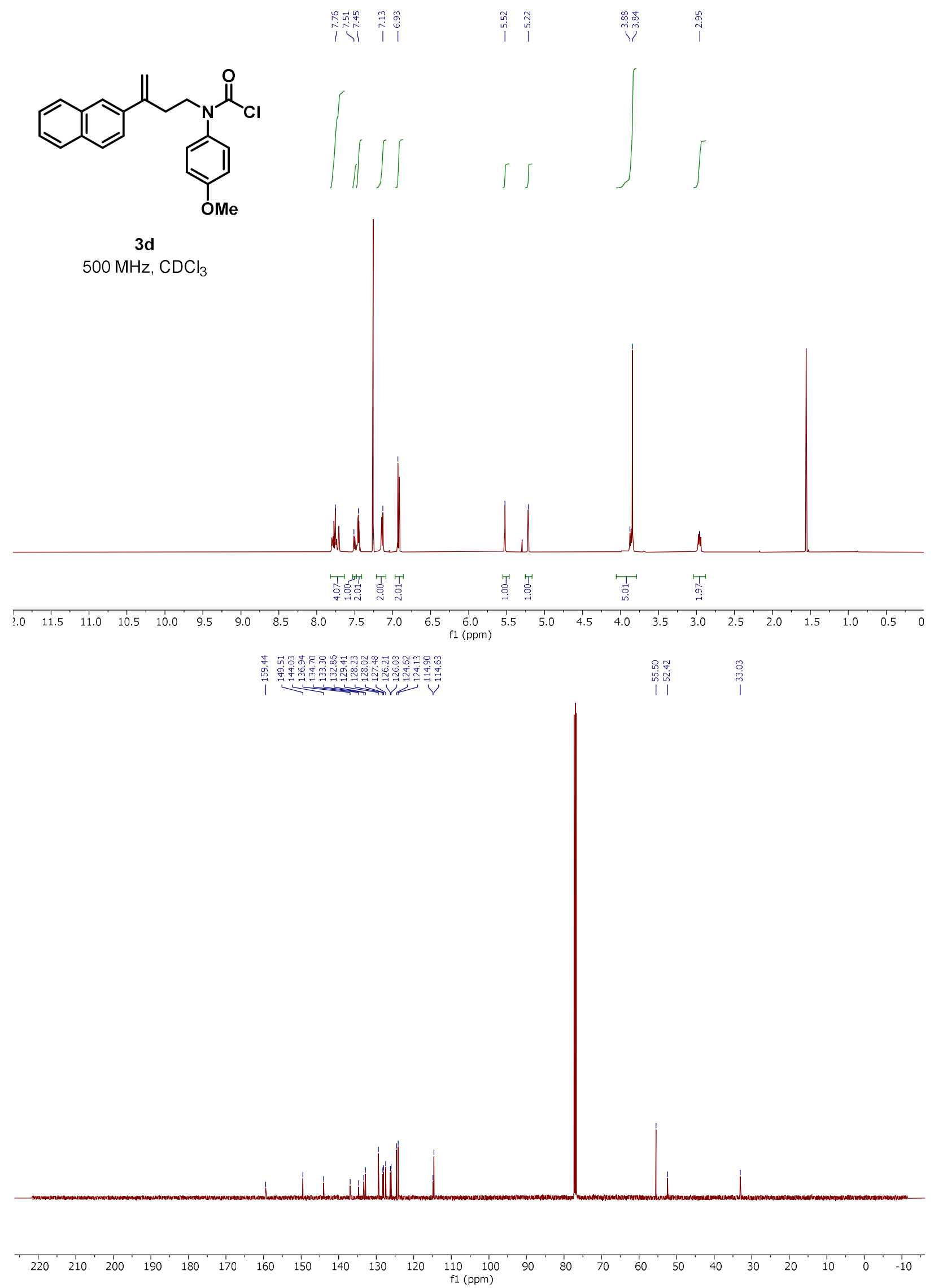

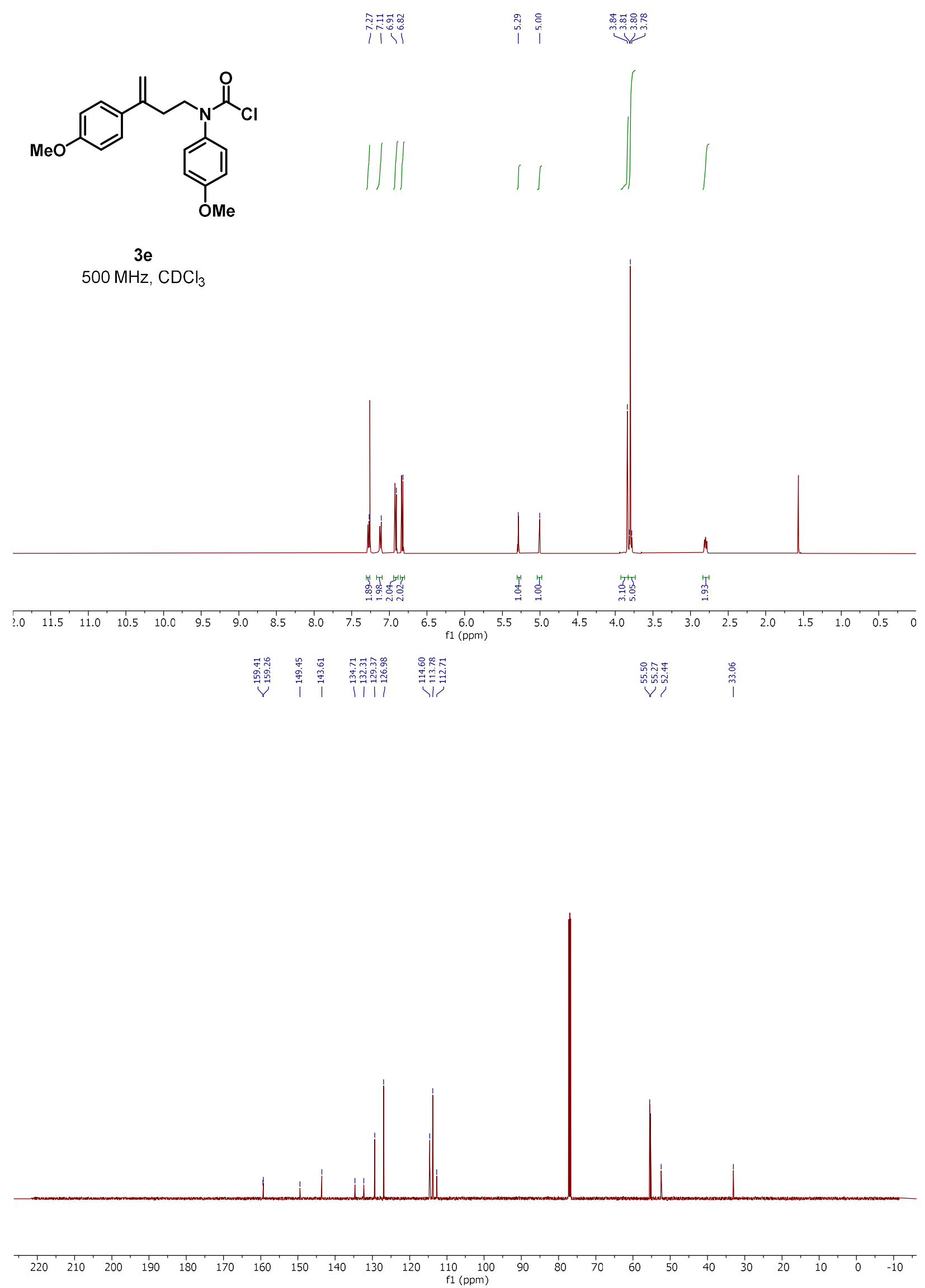


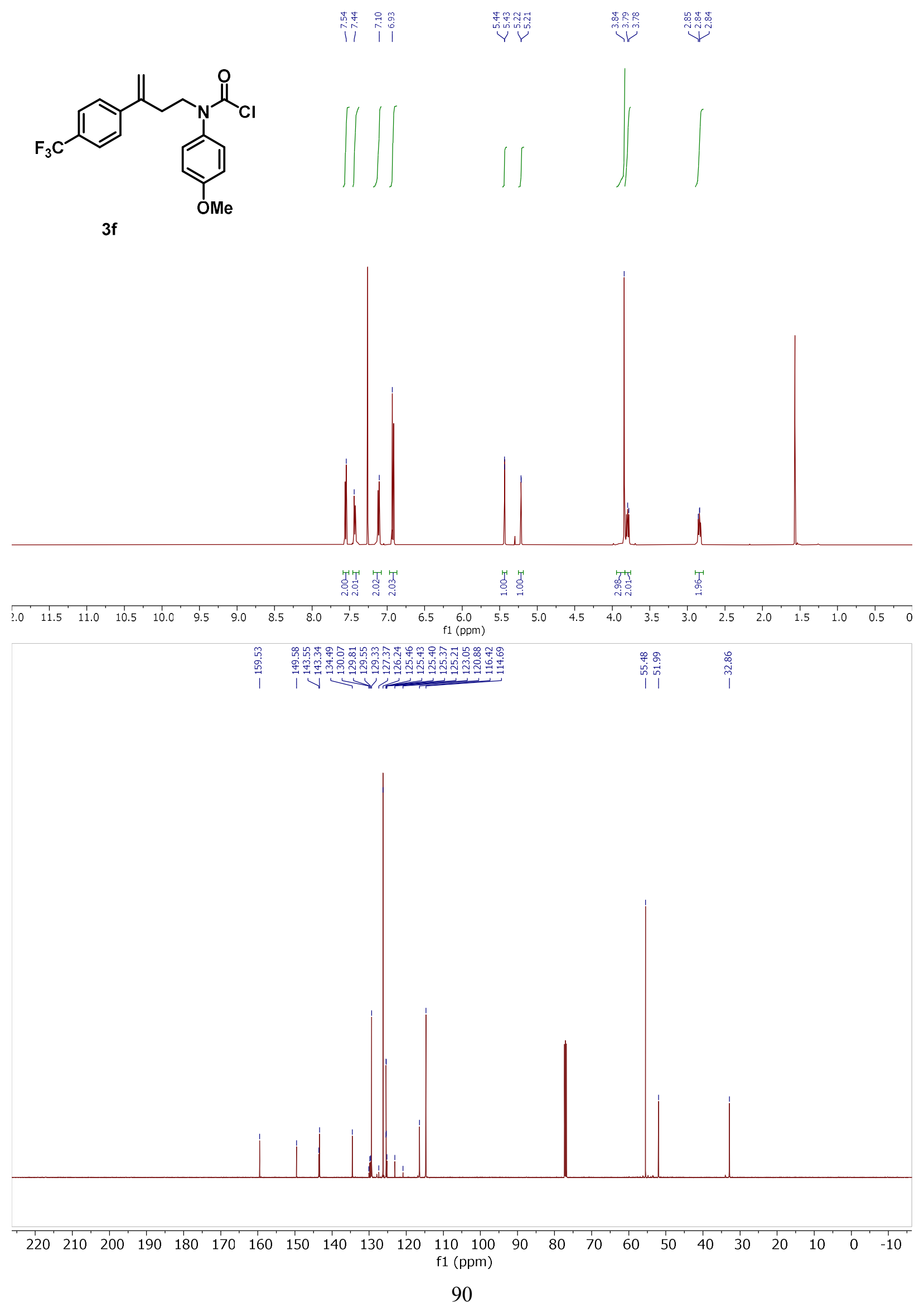


$400 \mathrm{MHz}, \mathrm{CDCl}_{3}$

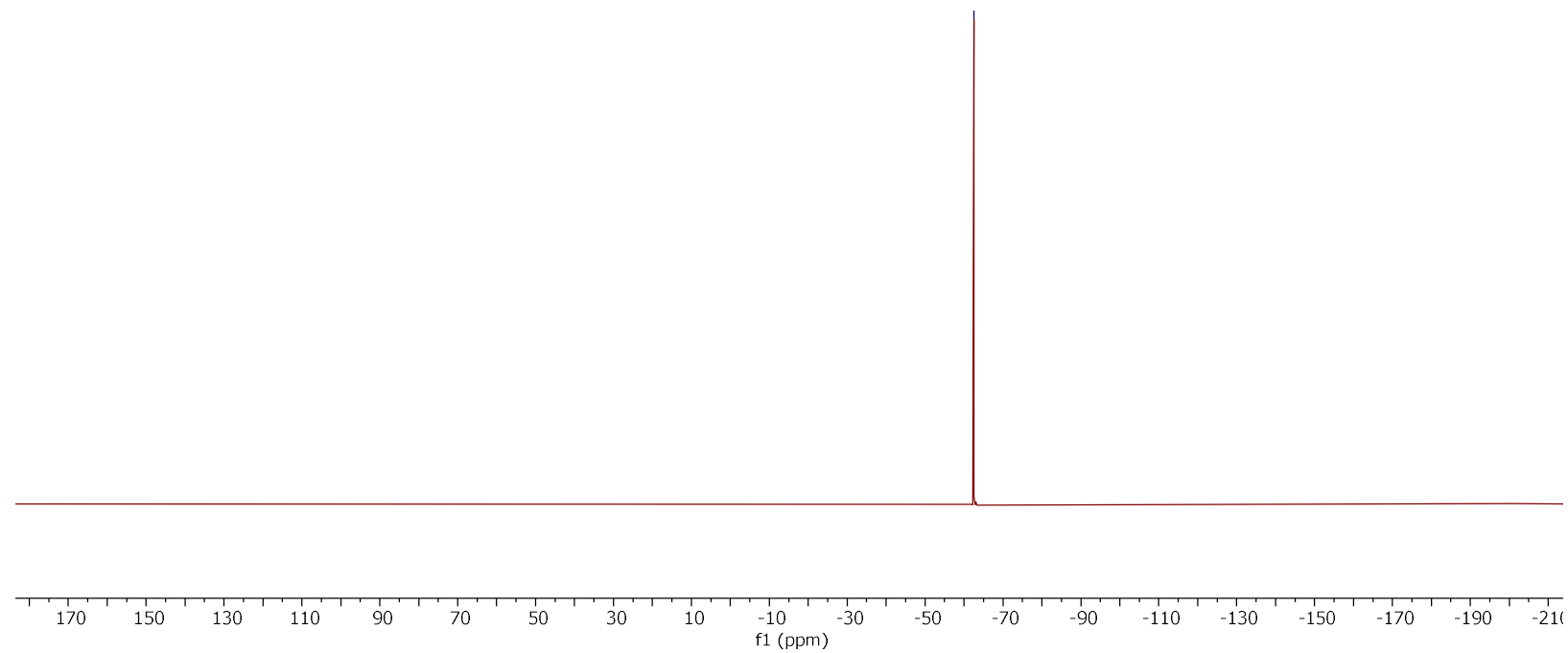




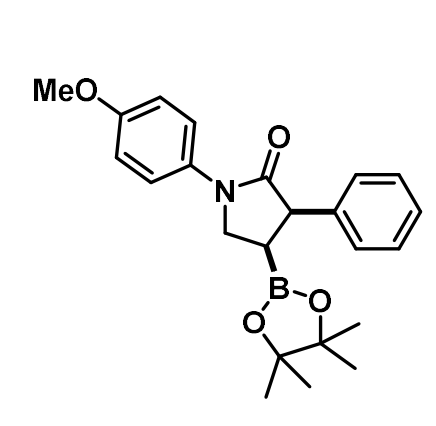

2a

$500 \mathrm{MHz}, \mathrm{CDCl}_{3}$

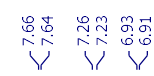
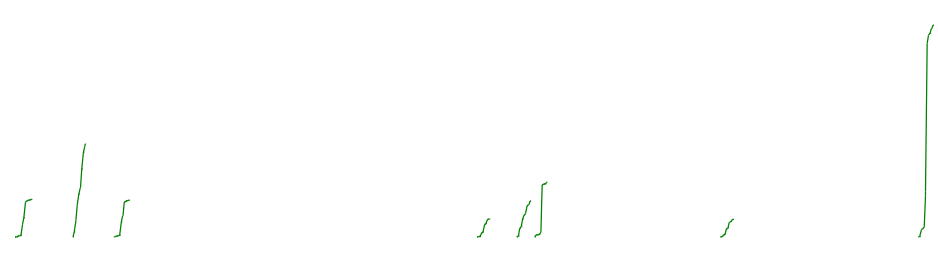

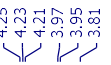

i

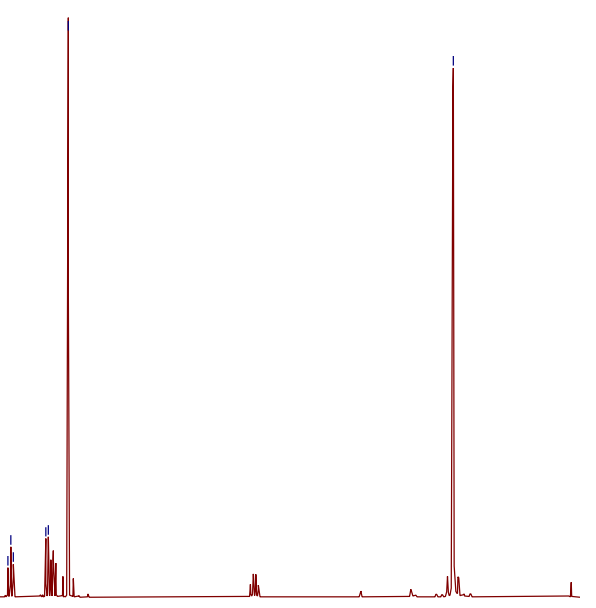

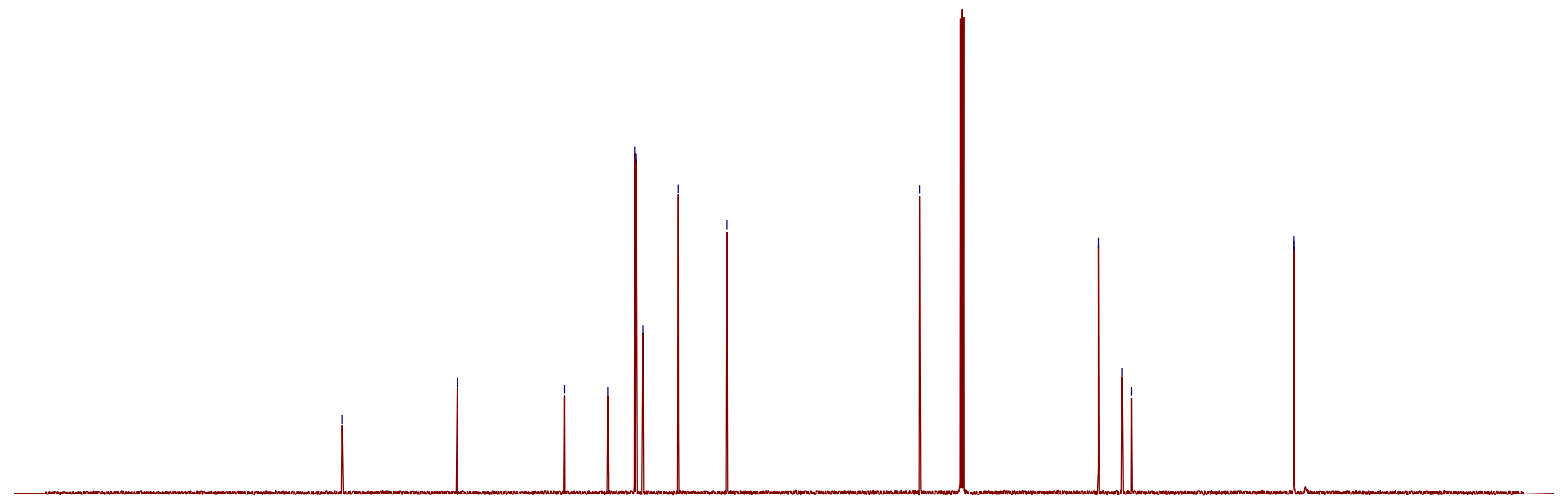

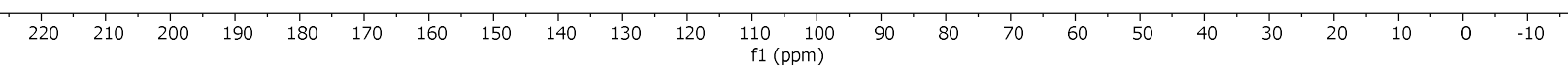



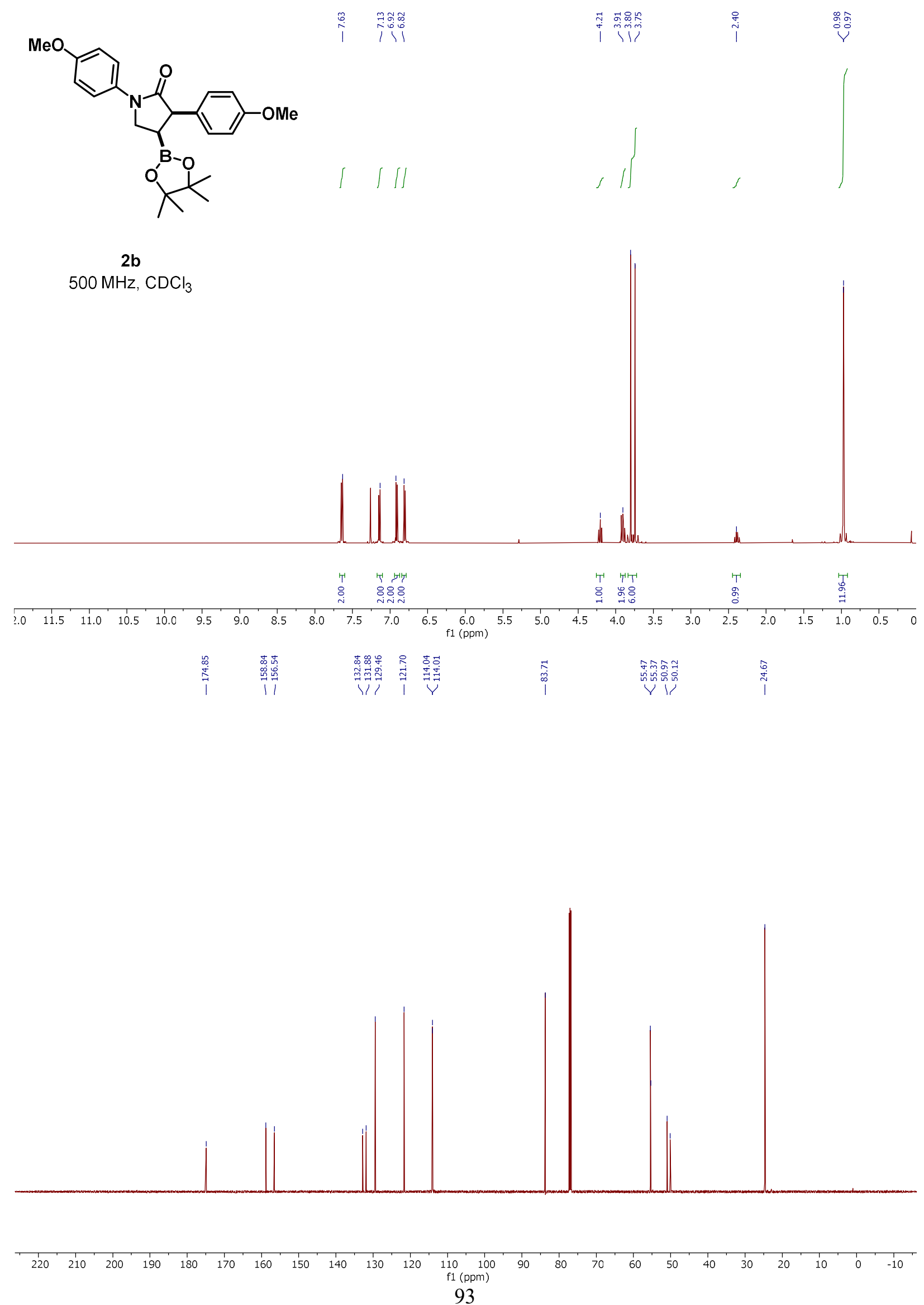


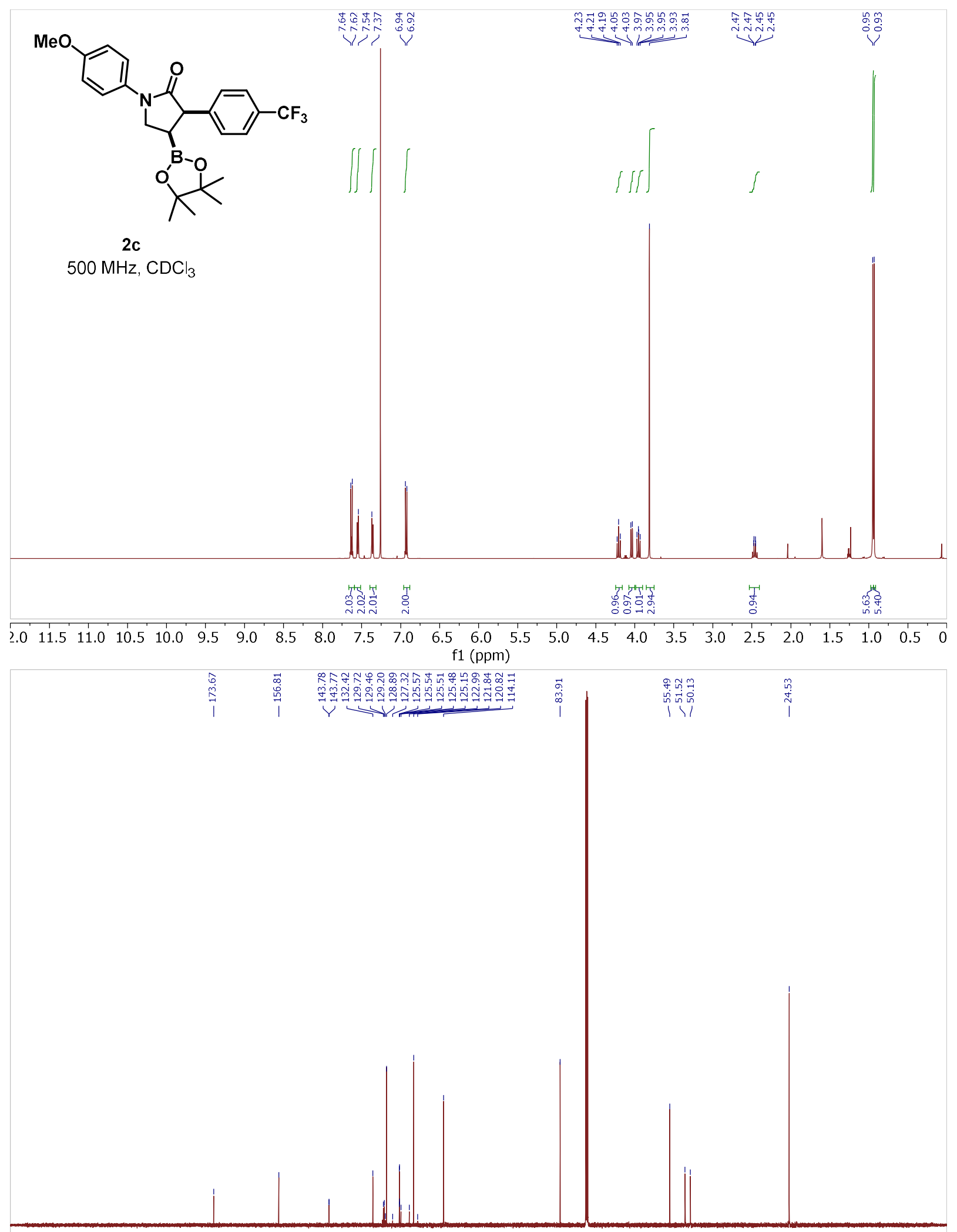

$\begin{array}{llllllllllllllllllllllll}220 & 210 & 200 & 190 & 180 & 170 & 160 & 150 & 140 & 130 & 120 & 110 & 100 & 90 & 80 & 70 & 60 & 50 & 40 & 30 & 20 & 10 & 0 & -10\end{array}$ 
$400 \mathrm{MHz}, \mathrm{CDCl}_{3}$

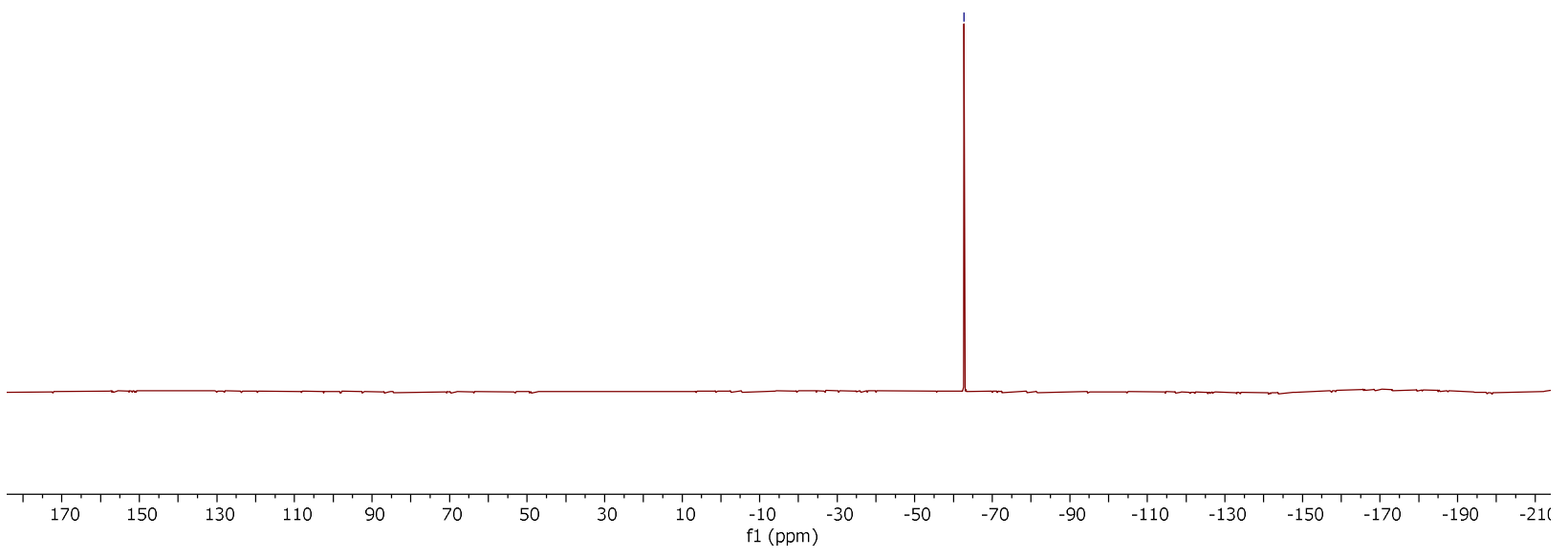




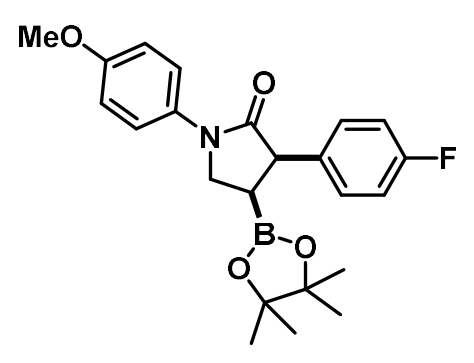

2d

$500 \mathrm{MHz}, \mathrm{CDCl}_{3}$
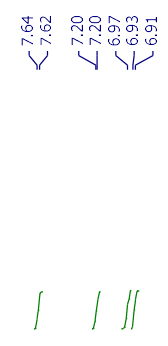

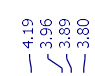

$\stackrel{\mathcal{Y}}{\mathcal{Y}}$

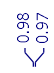

।रा।

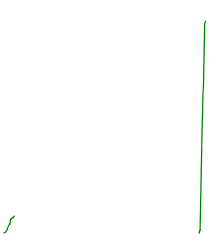

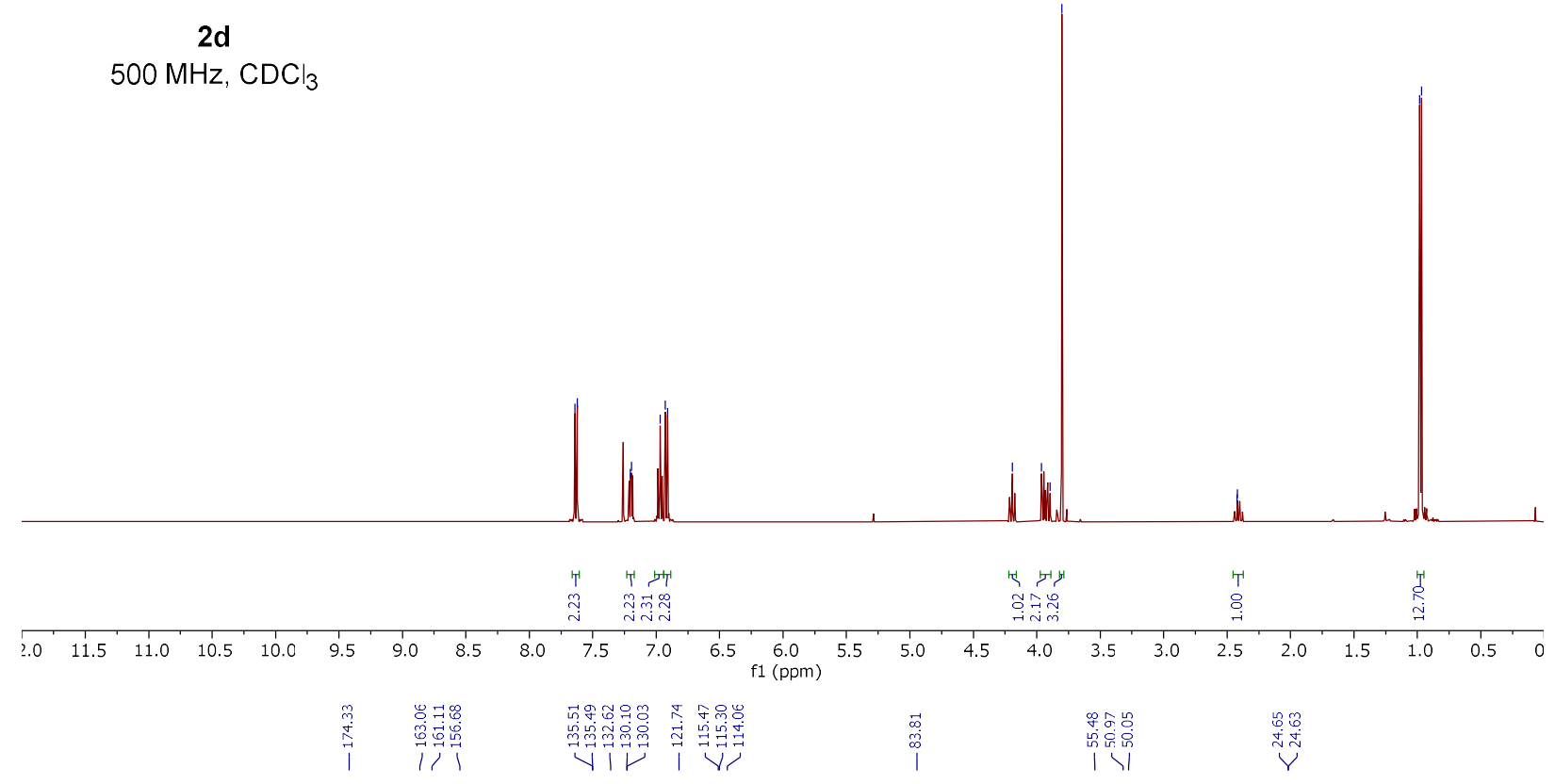
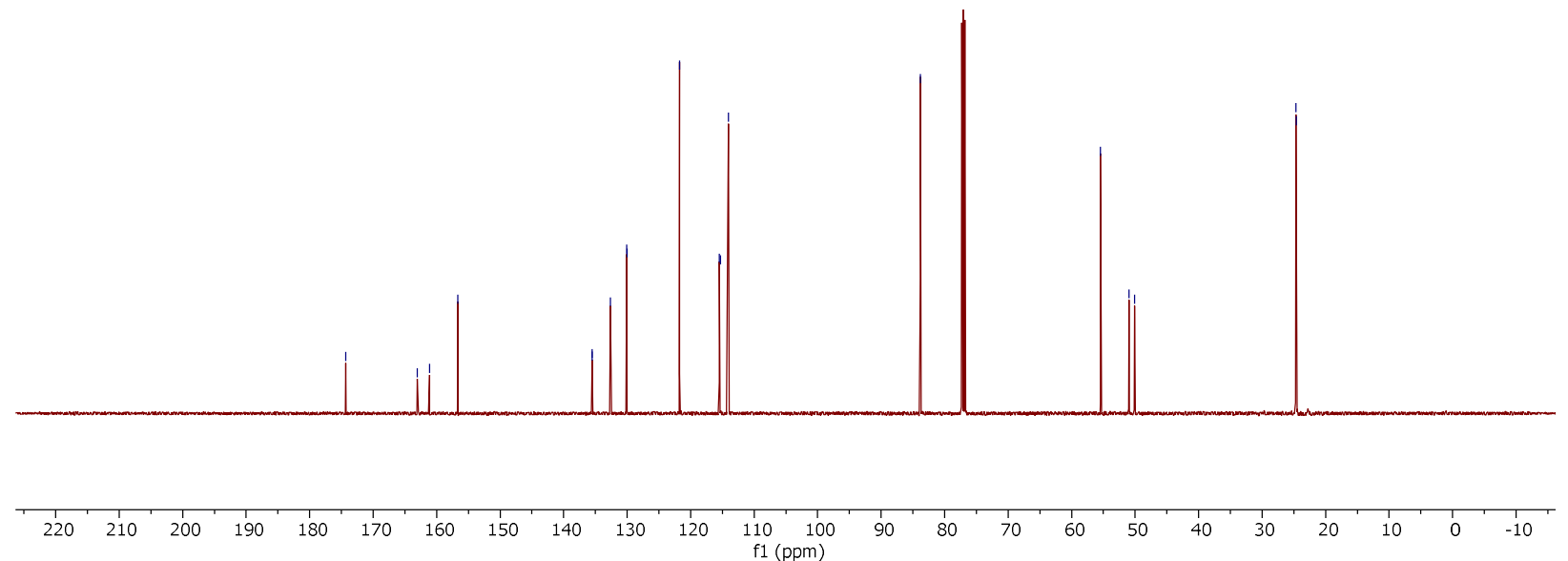
$400 \mathrm{MHz}, \mathrm{CDCl}_{3}$

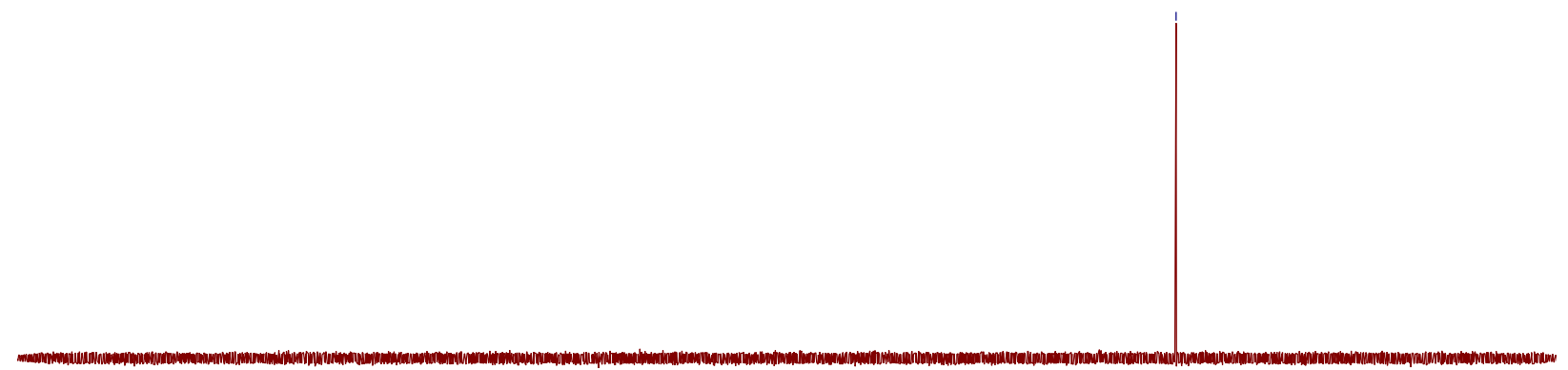

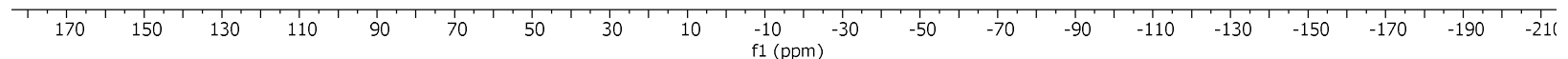



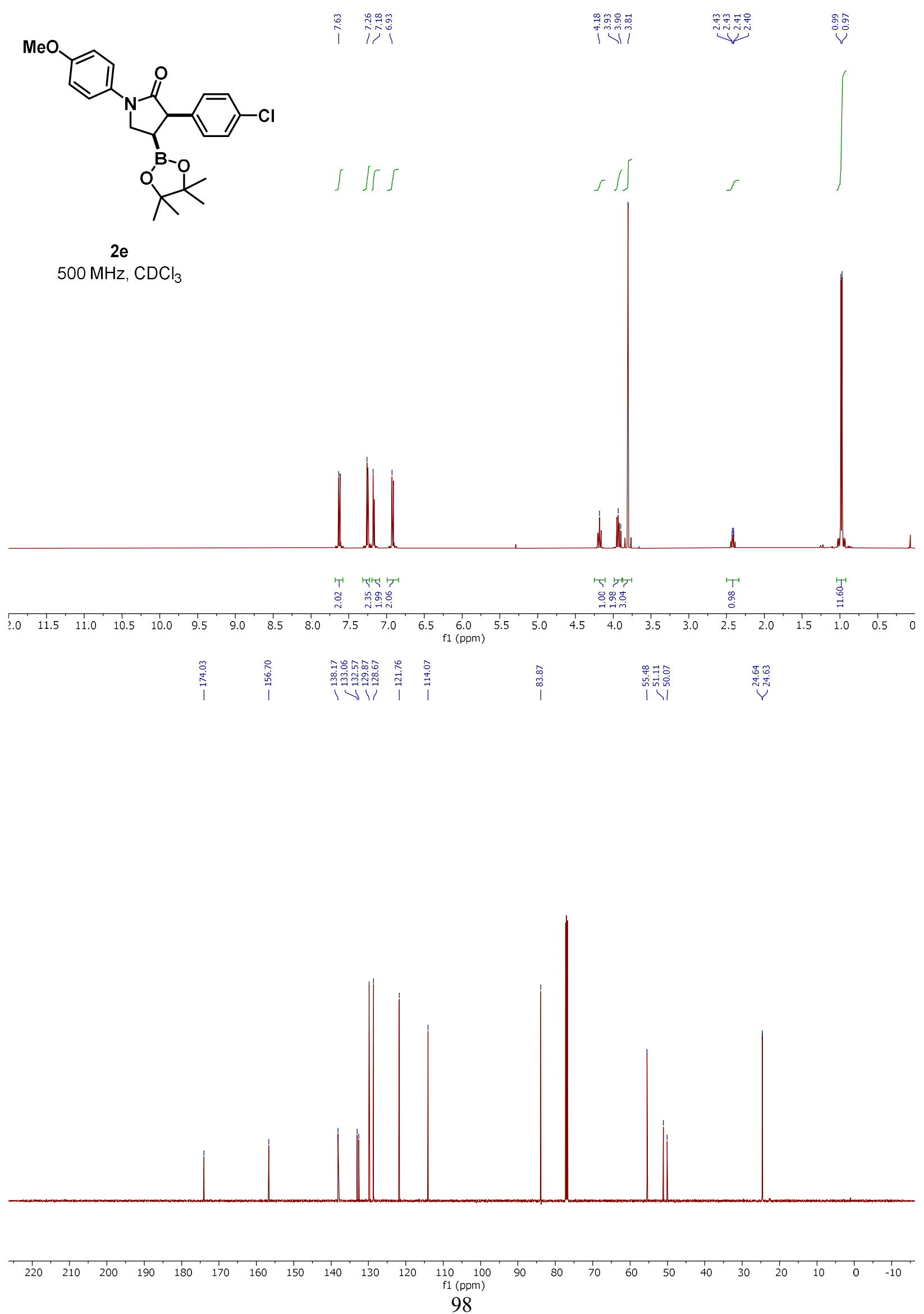

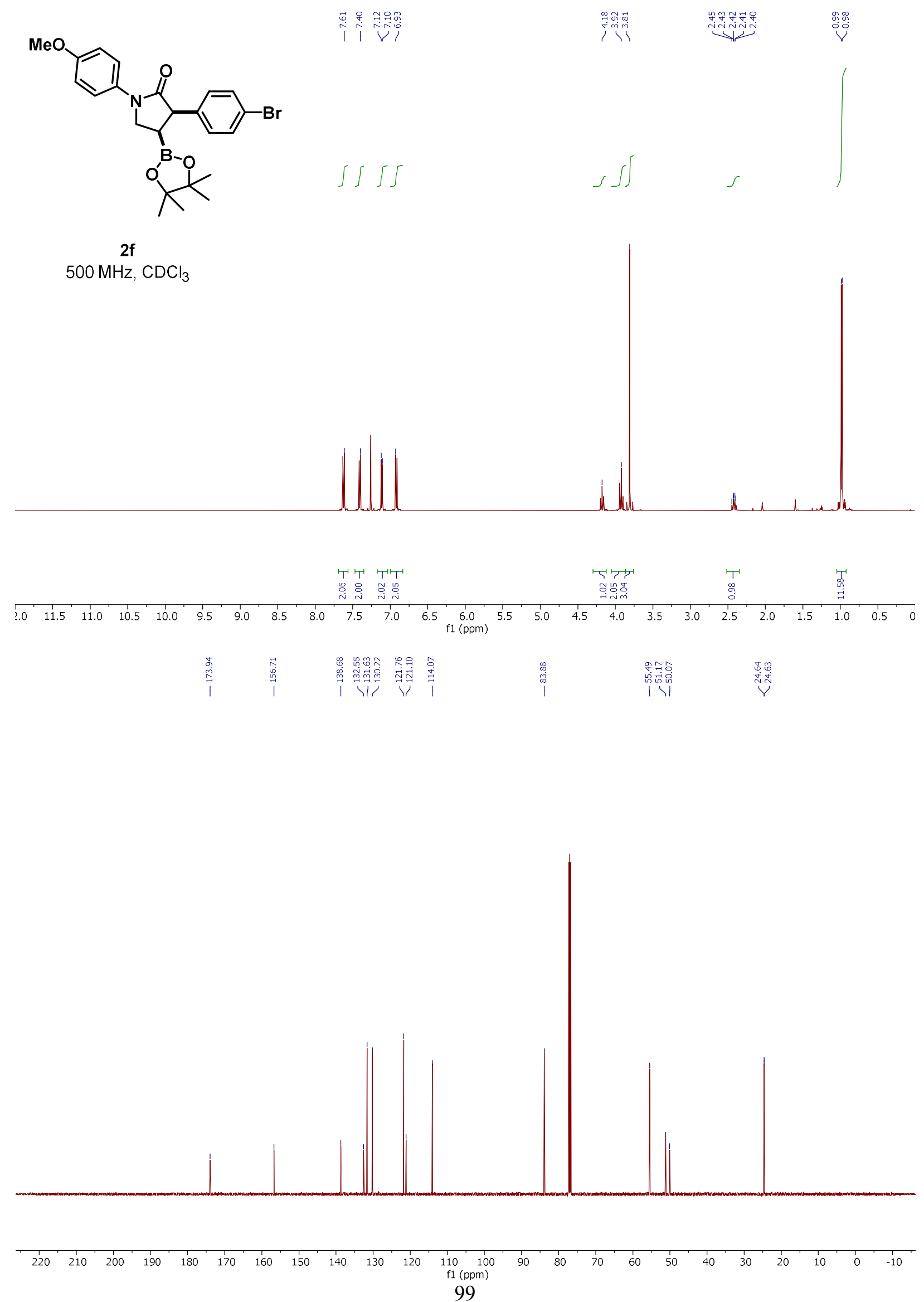


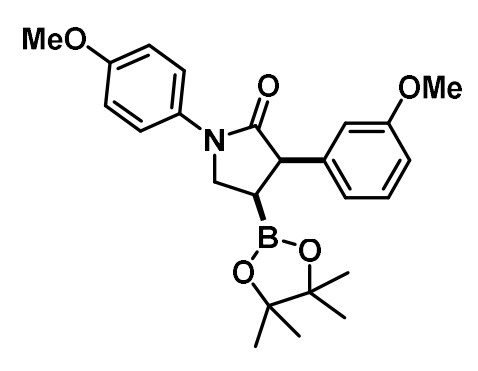

$2 \mathrm{~g}$

$500 \mathrm{MHz} \mathrm{CDCl}_{3}$
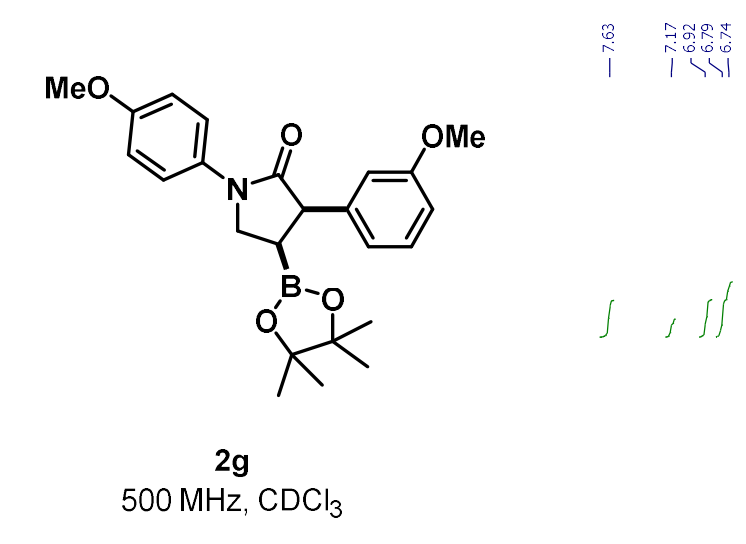
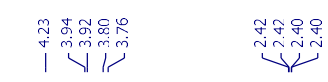

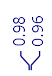
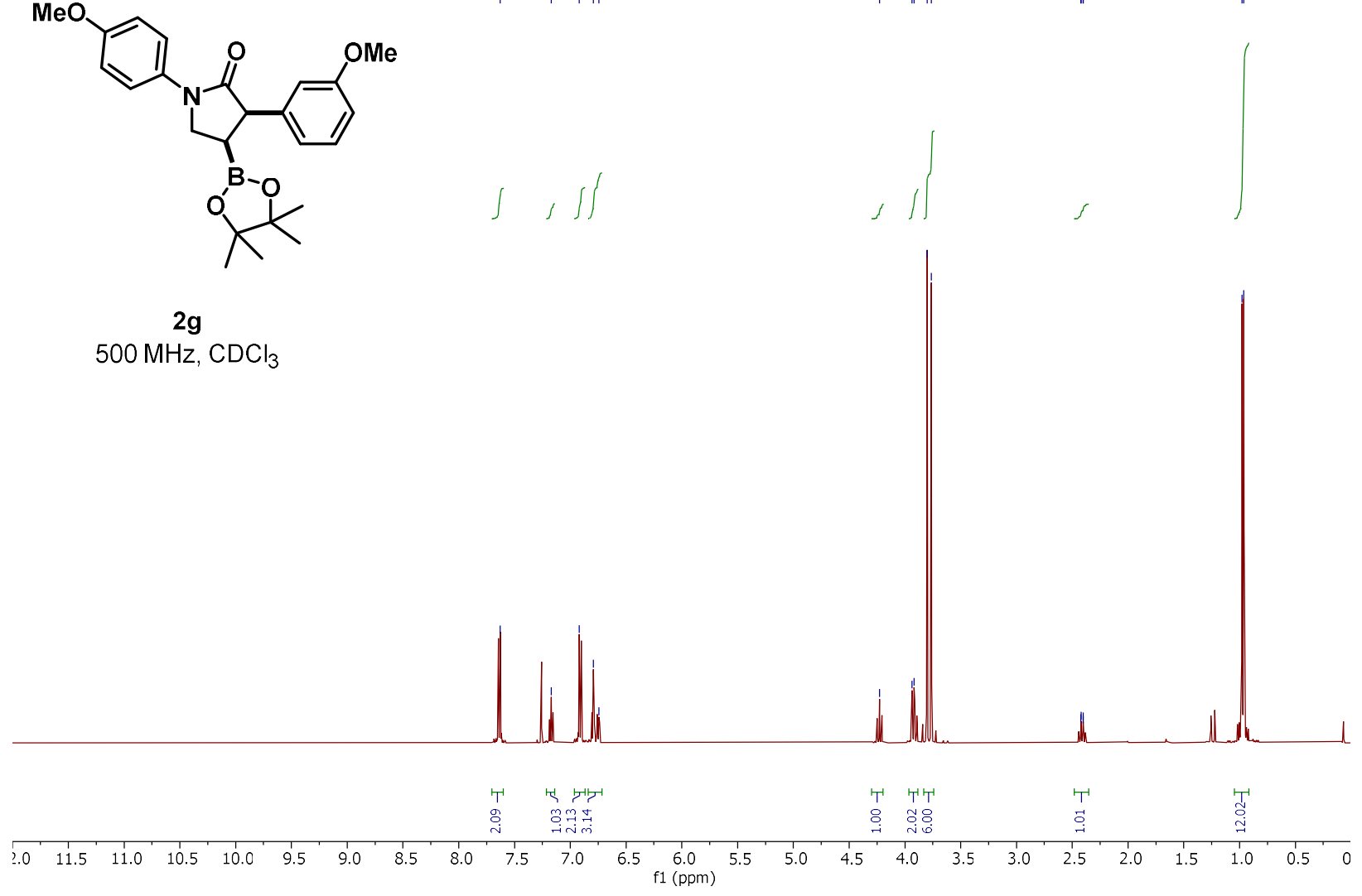

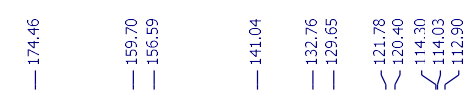
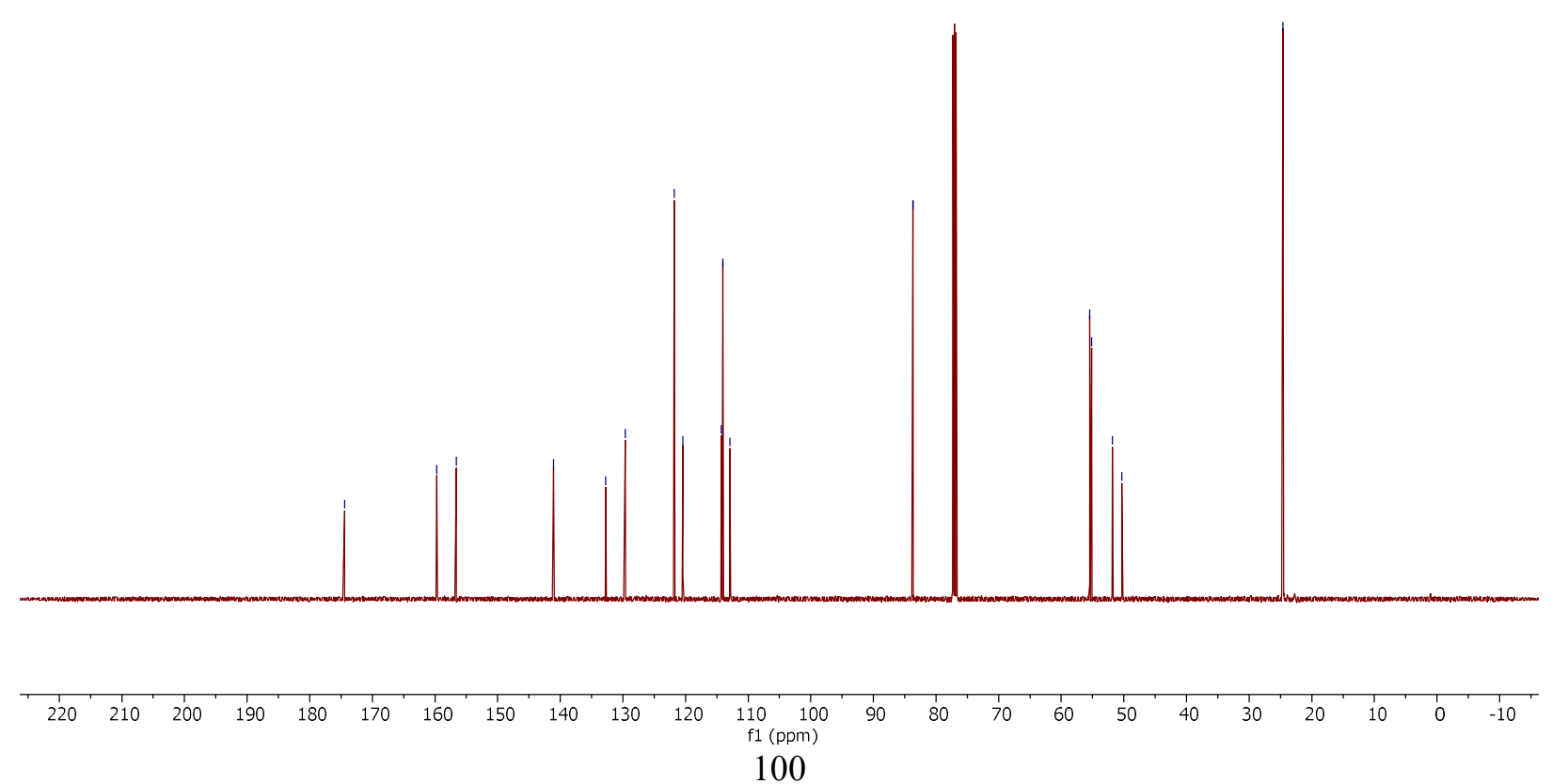

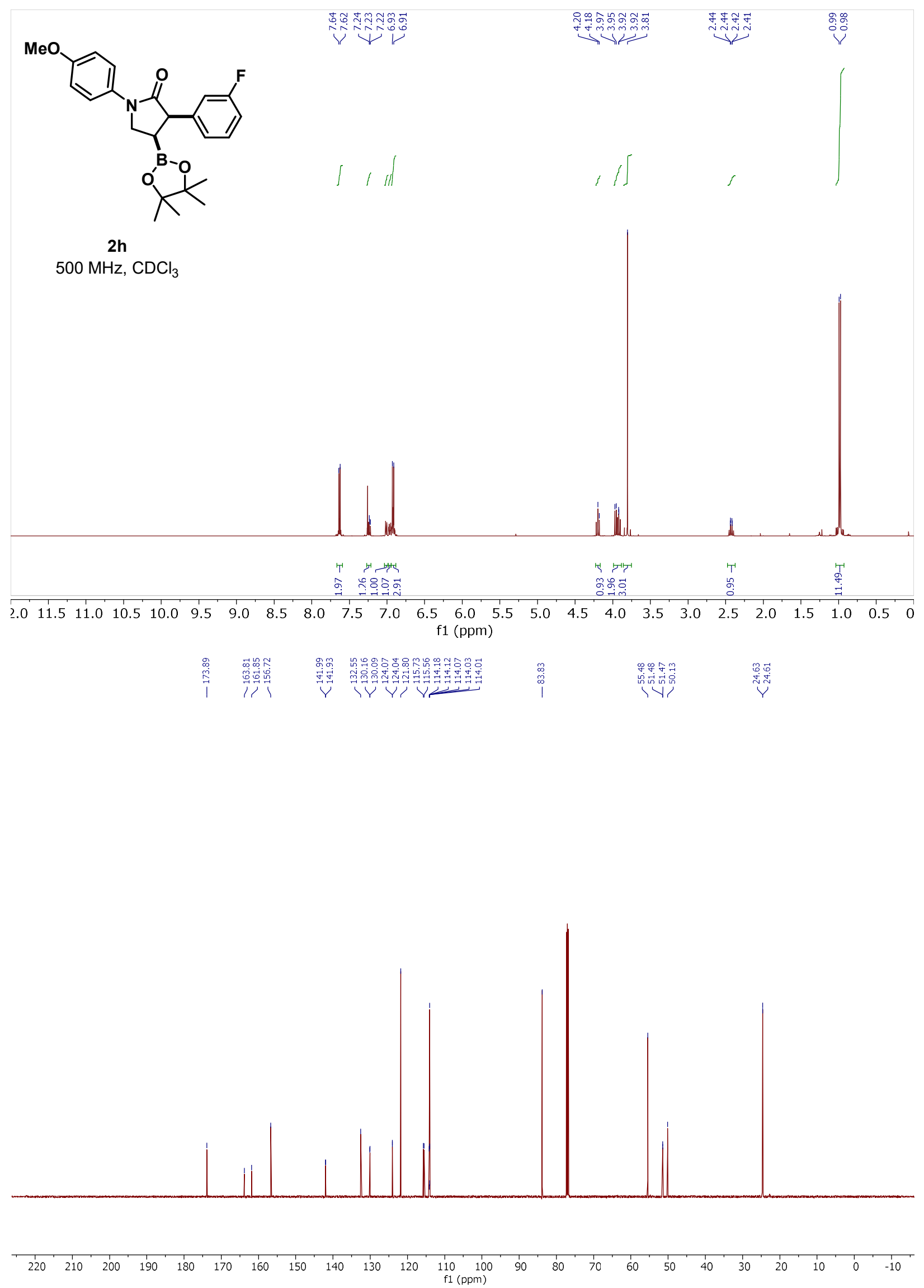
$400 \mathrm{MHz}, \mathrm{CDCl}_{3}$

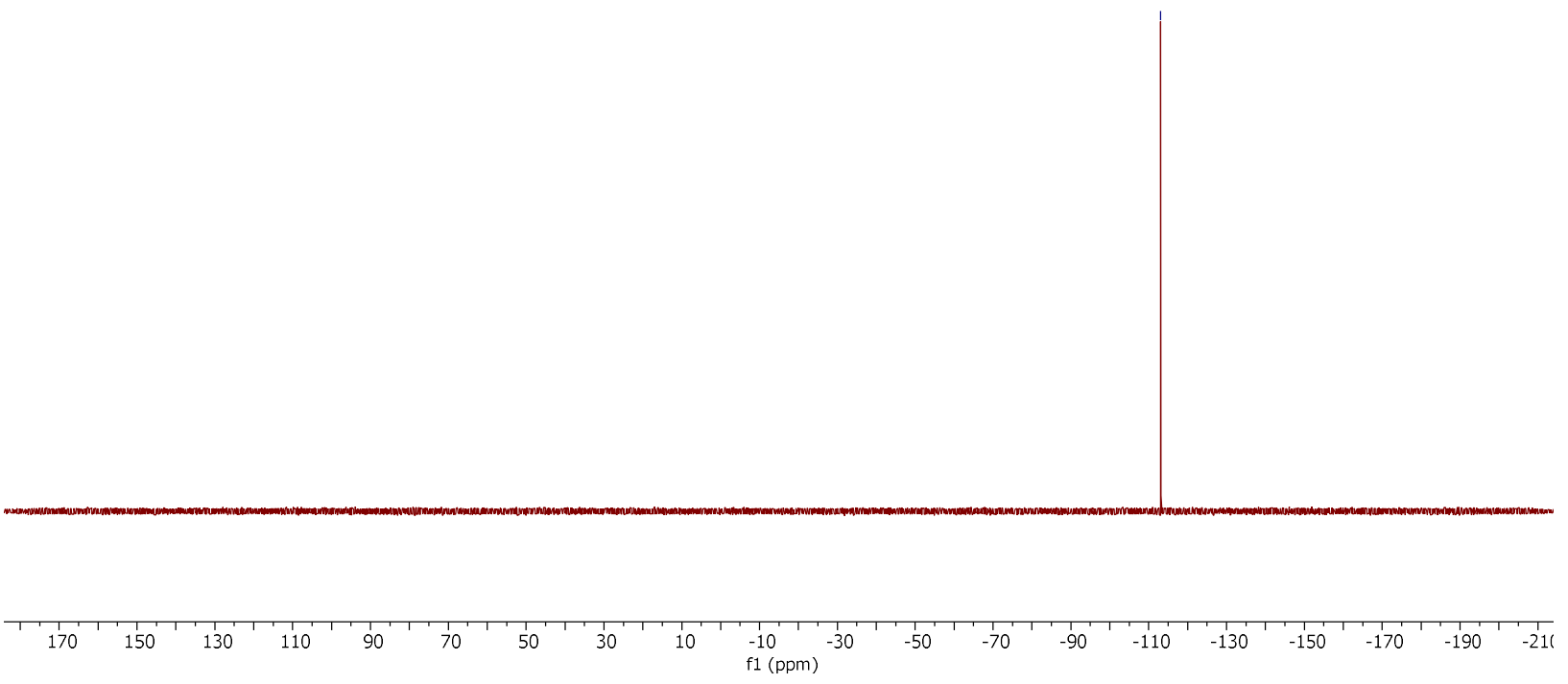




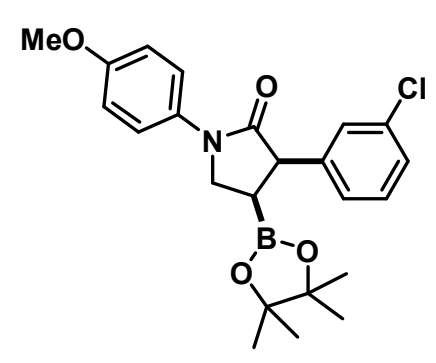

2

$500 \mathrm{MHz}, \mathrm{CDCl}_{3}$
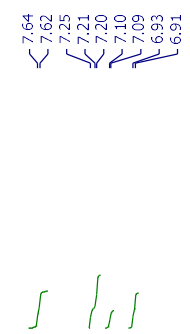
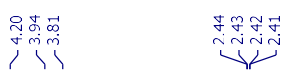

\&ic
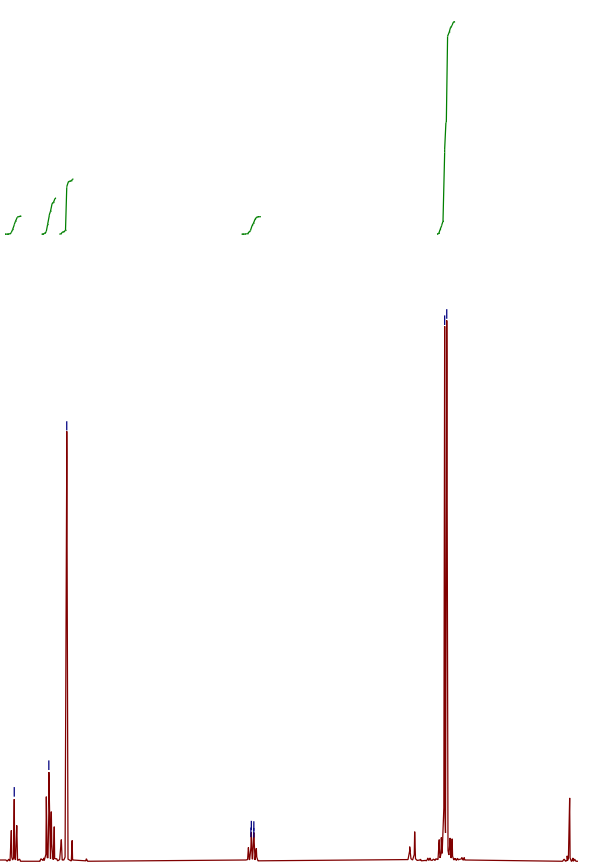

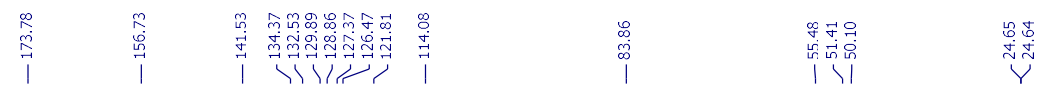

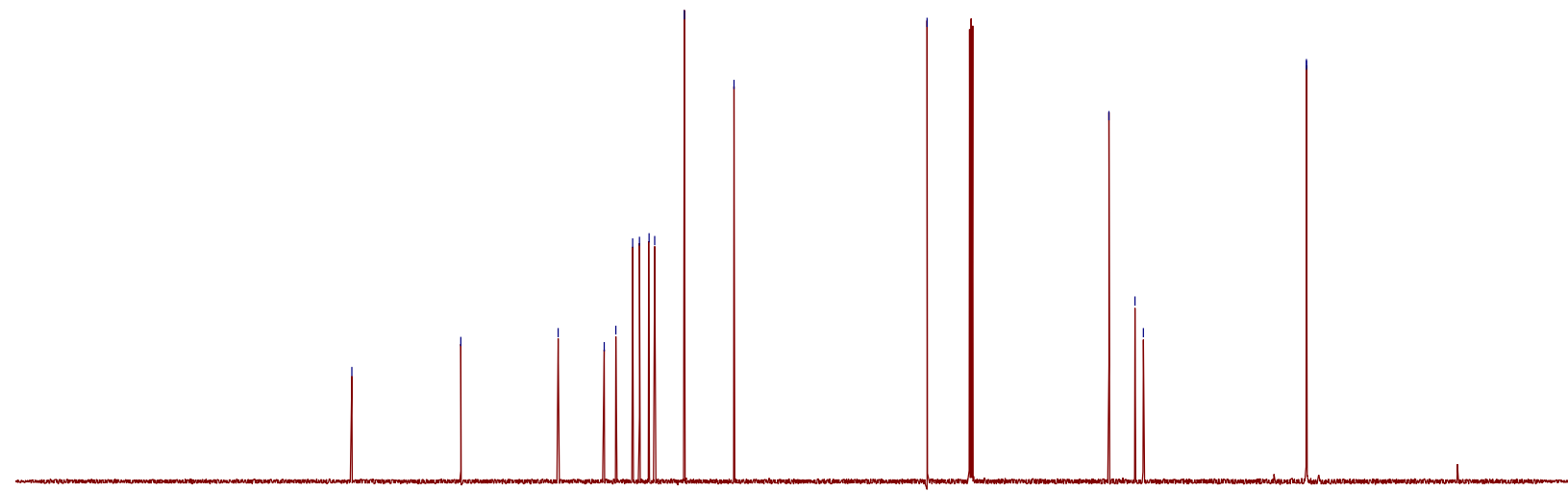

$\begin{array}{lllllllllllllllllllllllllllllllllll}1 & 220 & 210 & 200 & 190 & 180 & 170 & 160 & 150 & 140 & 130 & 120 & 110 & 100 & 90 & 80 & 70 & 60 & 50 & 40 & 30 & 20 & 10 & 0 & -10\end{array}$ 


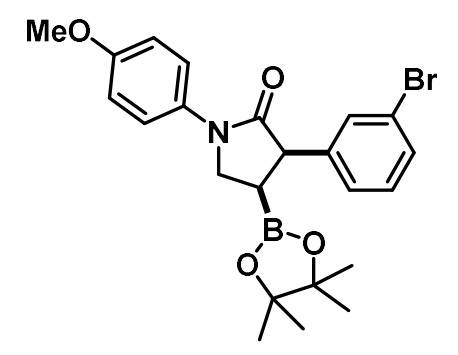

2j

$500 \mathrm{MHz}, \mathrm{CDCl}_{3}$ $\iiint \int$

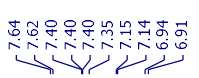

Ir

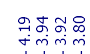

रोग

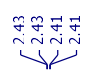

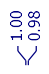

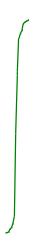

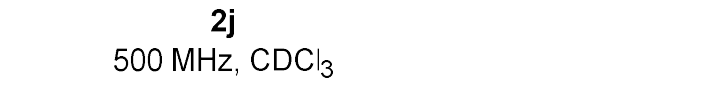
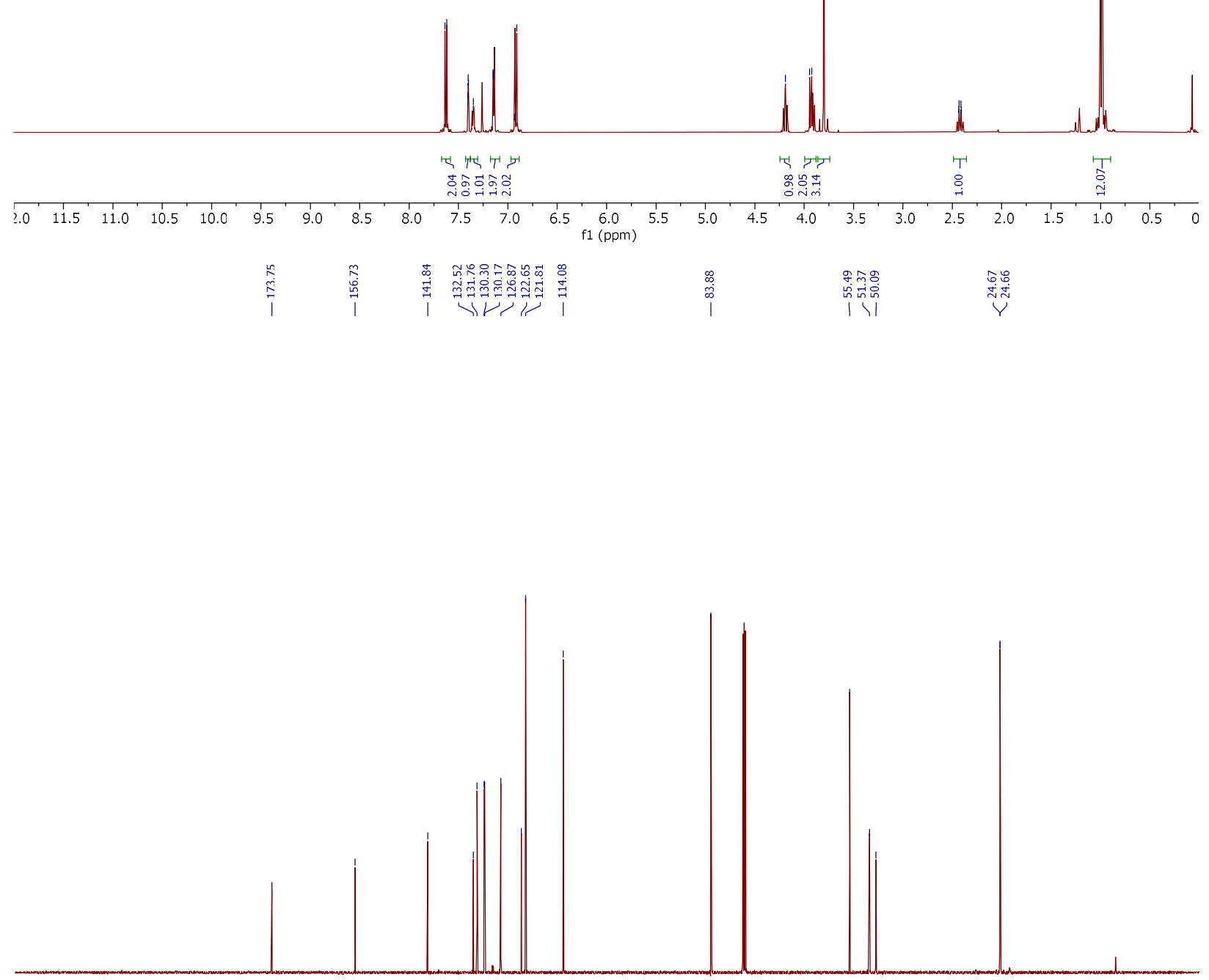

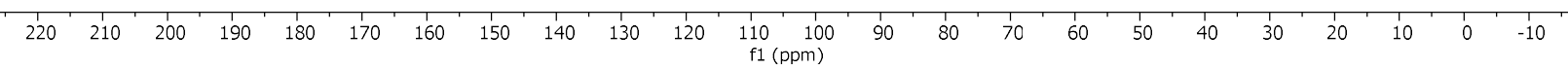
104 


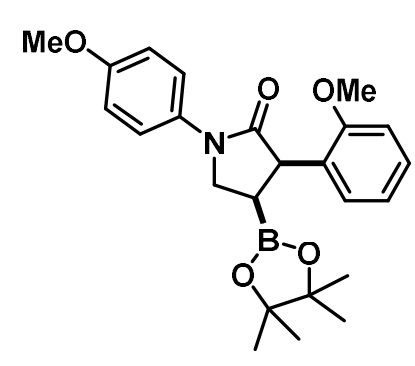

2k

$500 \mathrm{MHz}, \mathrm{CDCl}_{3}$
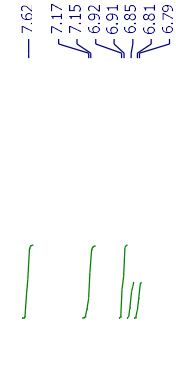

|

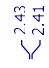
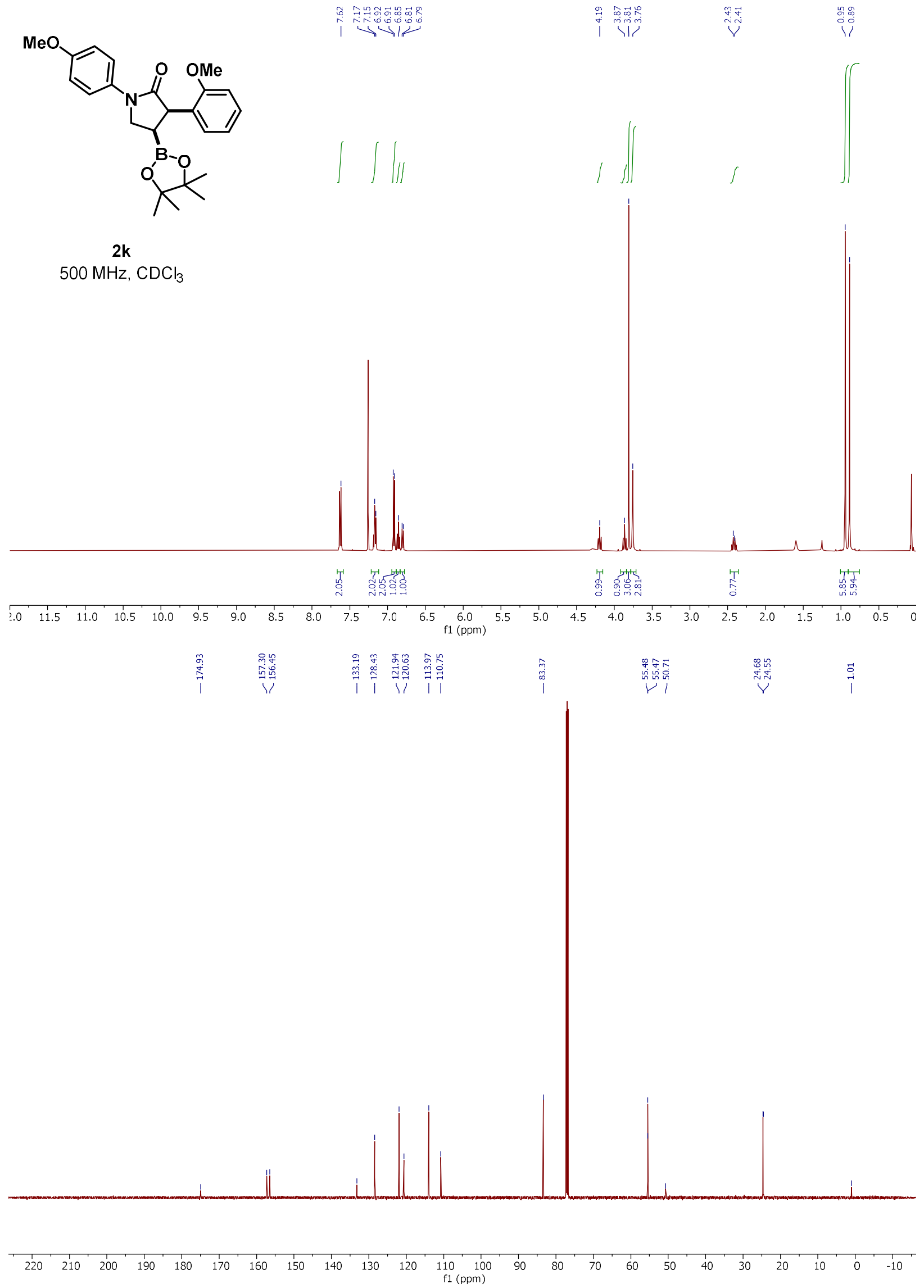

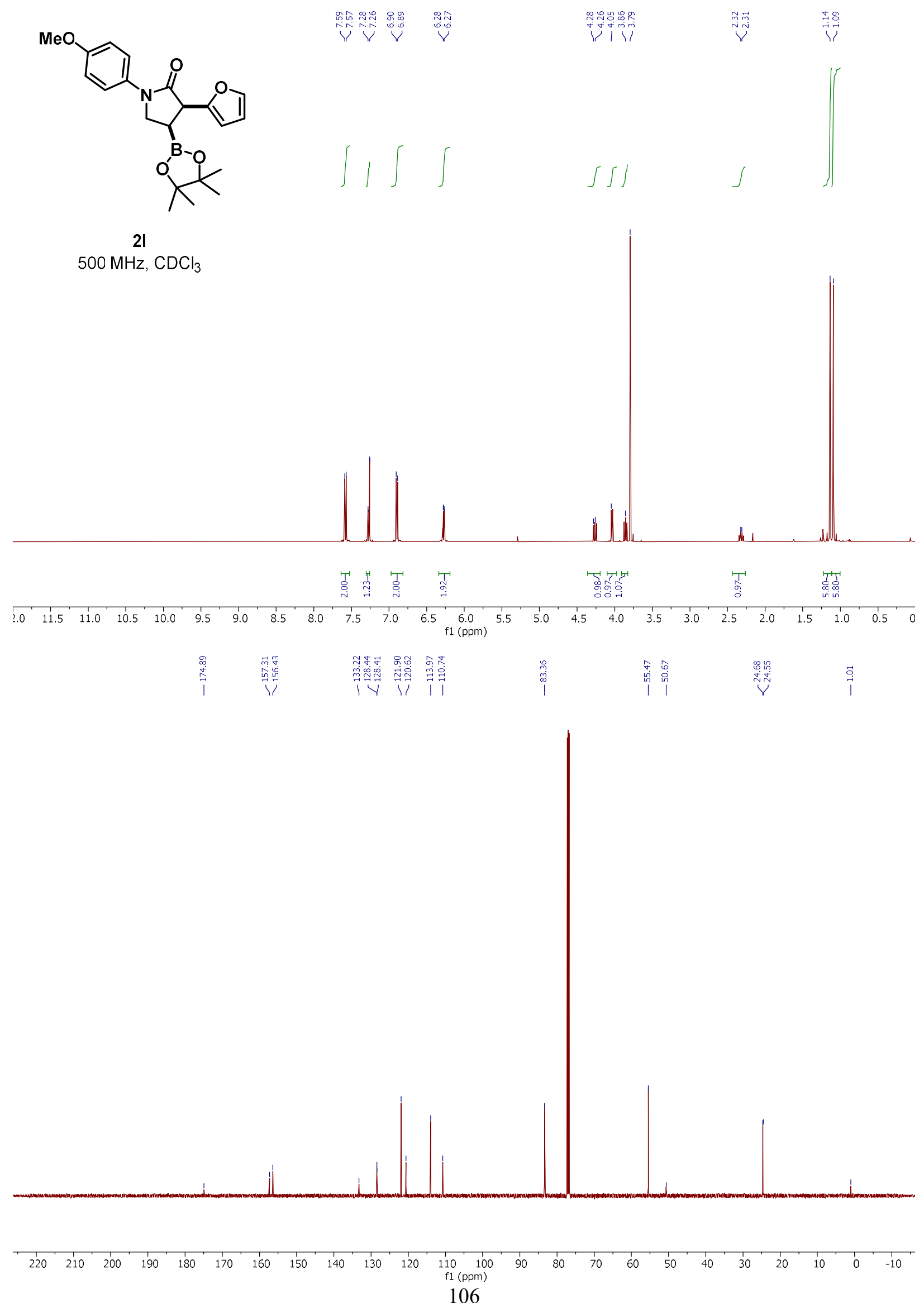

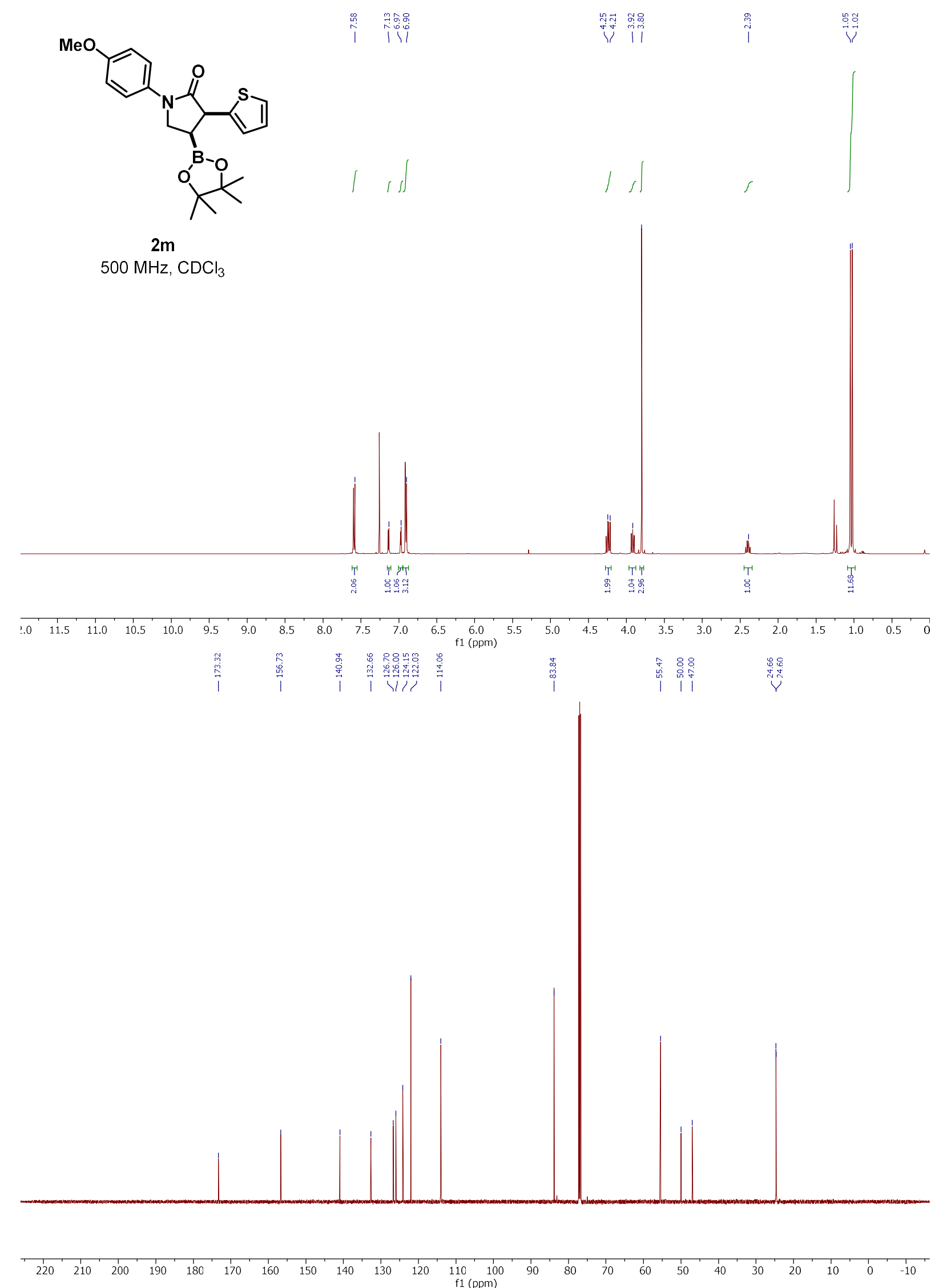


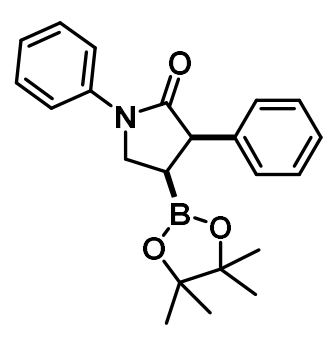

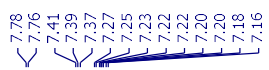

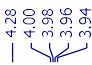

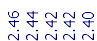

Y.

2n

$500 \mathrm{MHz} \mathrm{CDCl}_{3}$
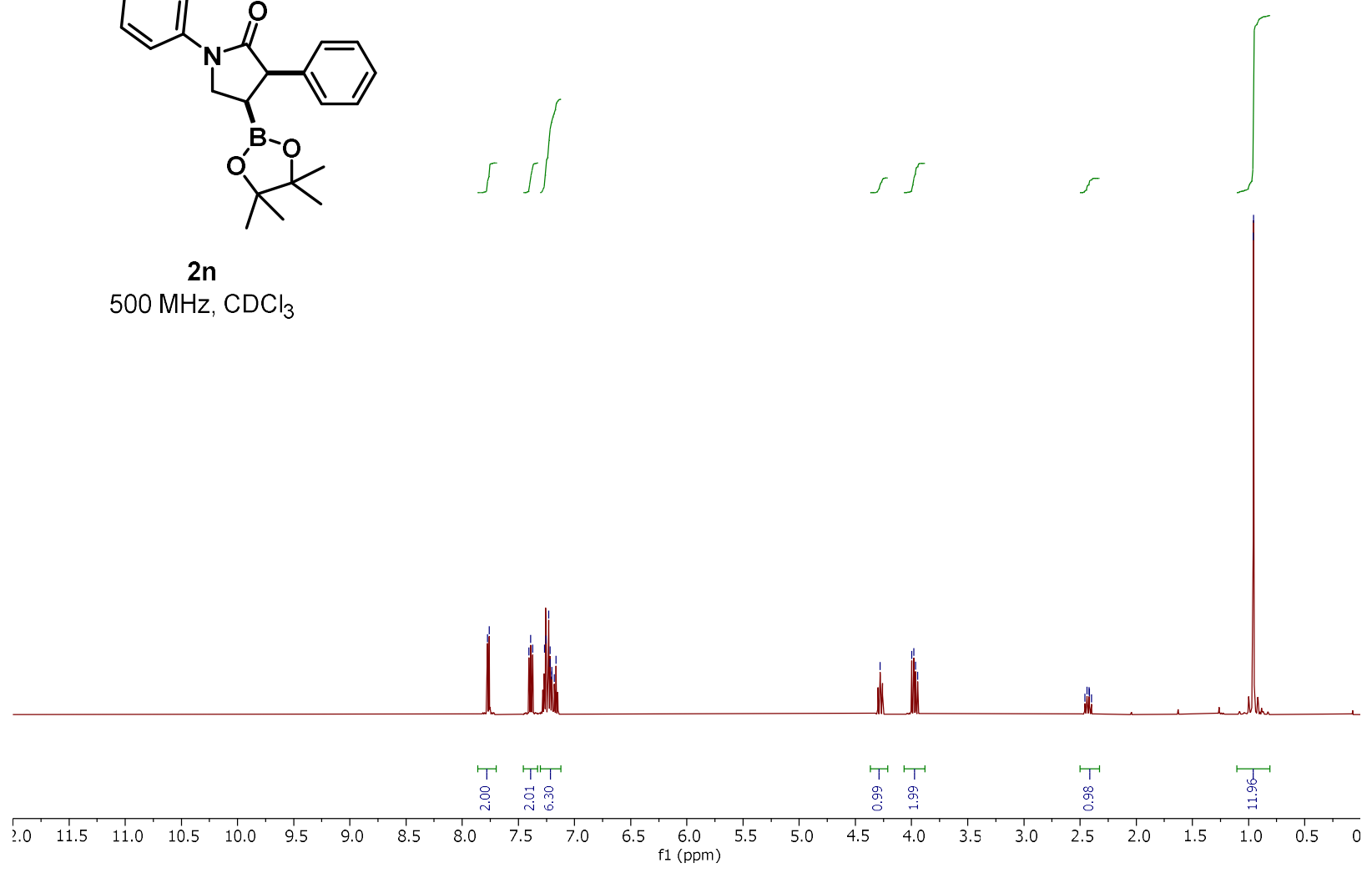

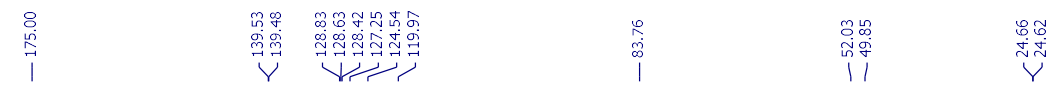
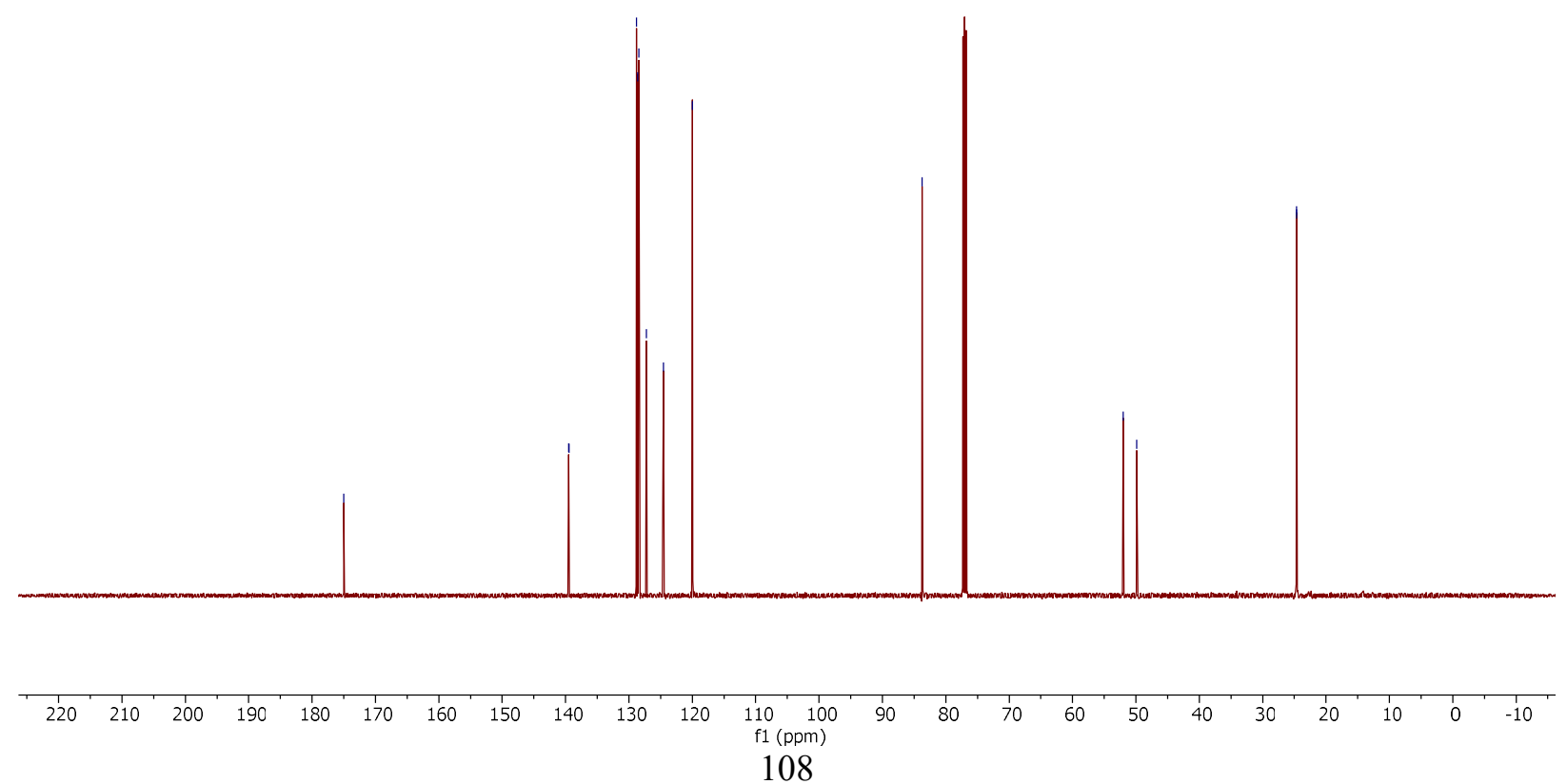
Me<smiles>CC1(C)OB(C2CN(c3ccc(F)cc3)C(=O)C2c2ccccc2)OC1(C)C</smiles>

20

$500 \mathrm{MHz}, \mathrm{CDCl}_{3}$
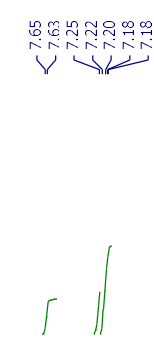

$5 \int$

|

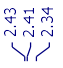
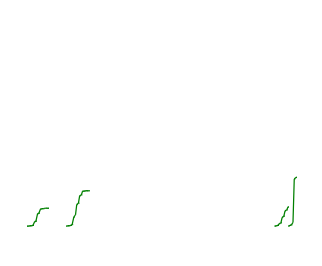

1
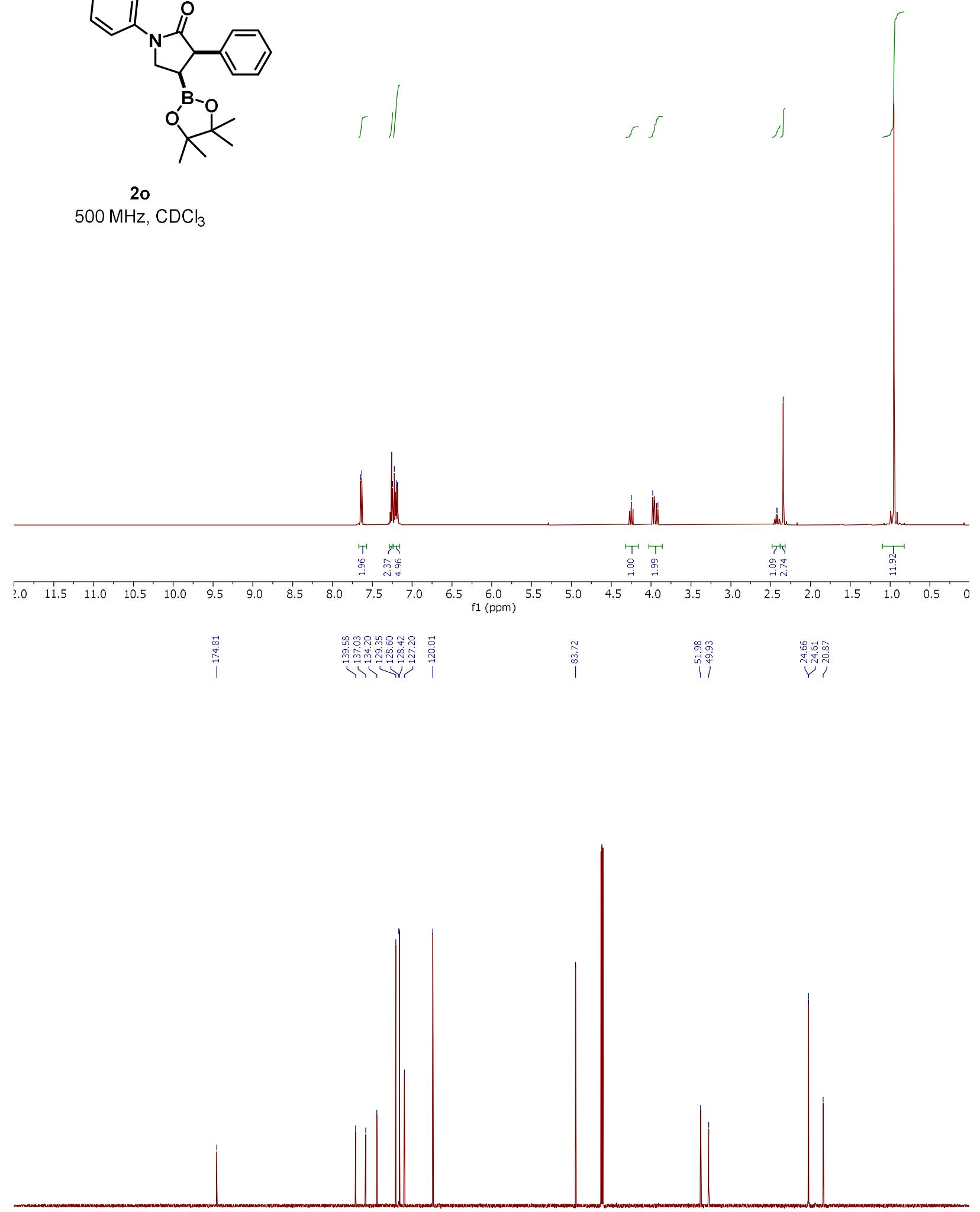

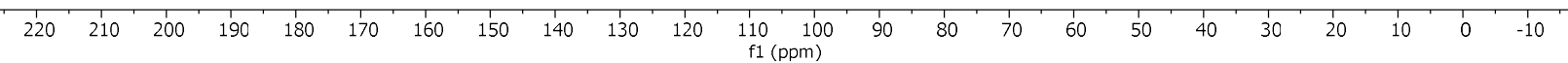



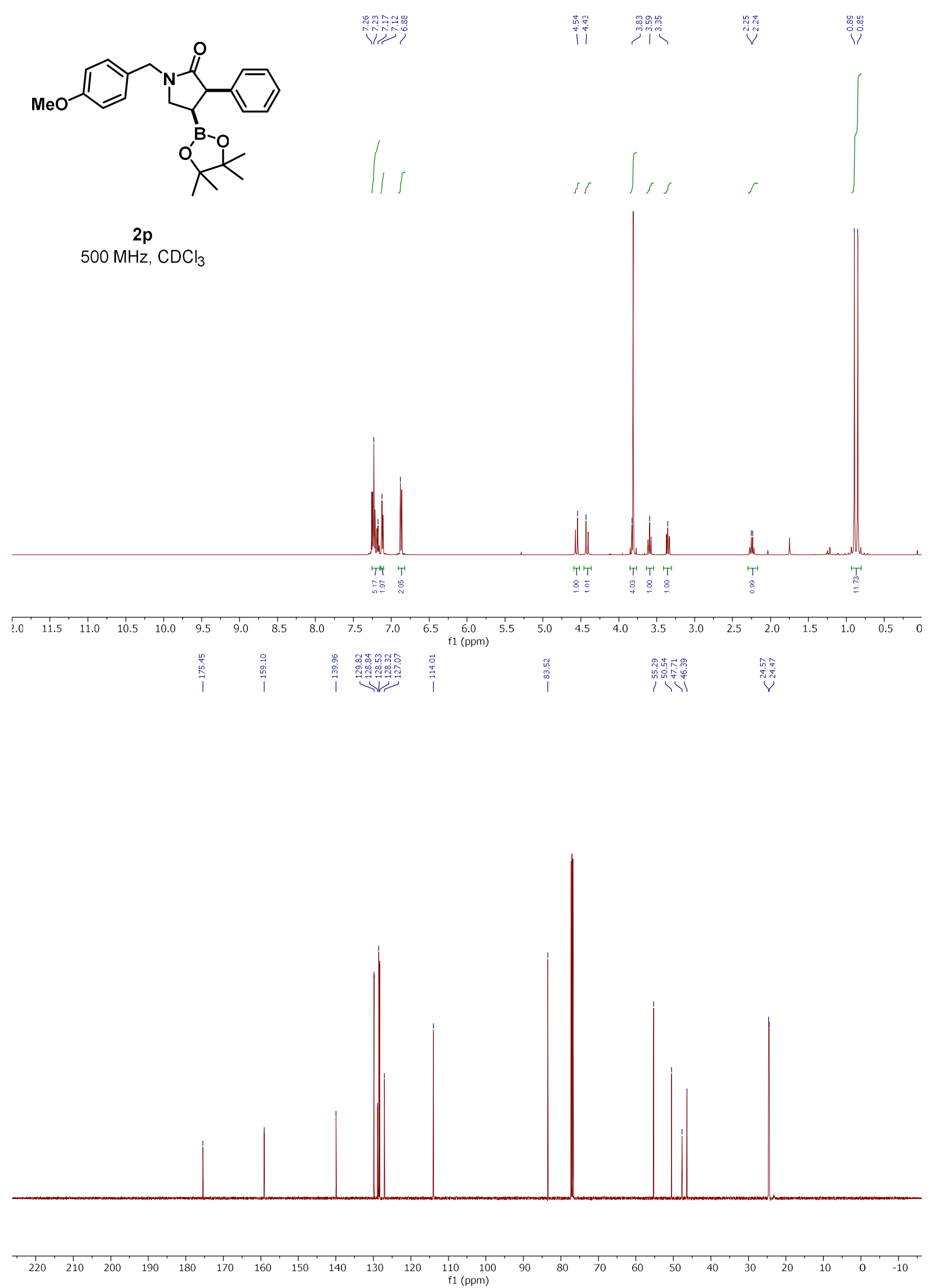

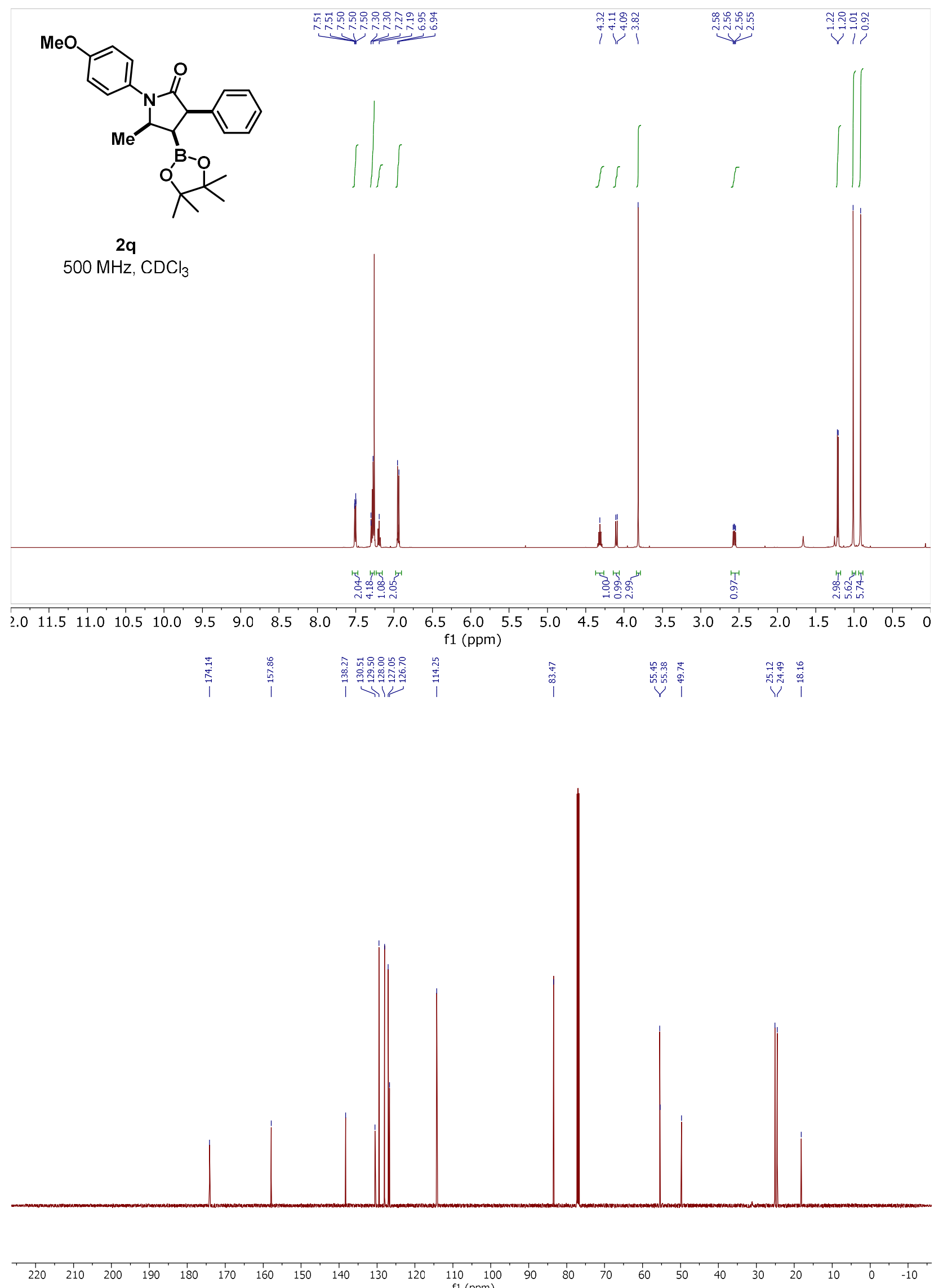

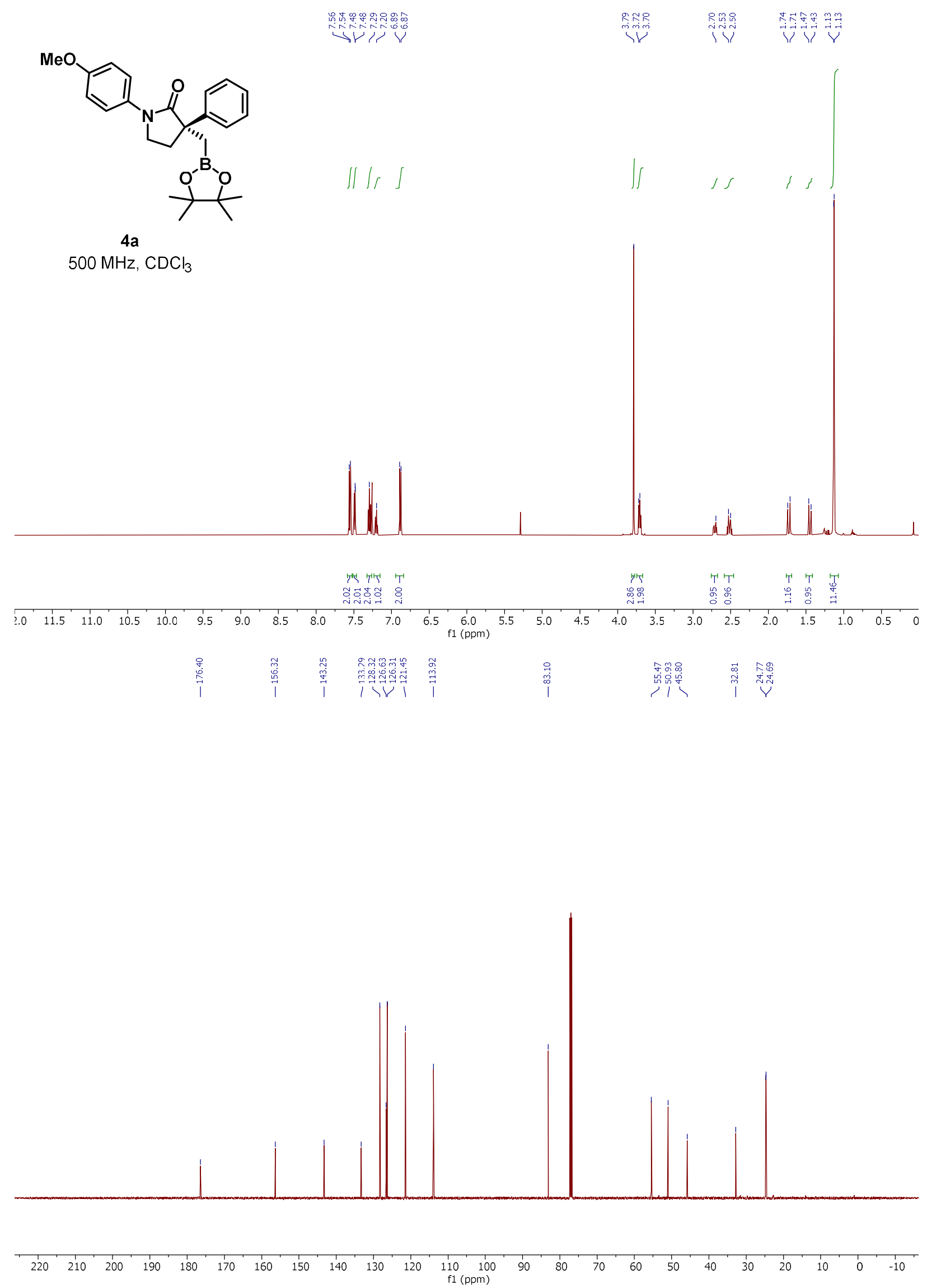
$\mathrm{MeO}$<smiles>COc1ccccc1C1(C(=O)N2CCCN(c3ccccc3)C2=O)CO[PH](OC)(C(C)(C)C)C1(C)C</smiles>

4b

$500 \mathrm{MHz}, \mathrm{CDCl}_{3}$
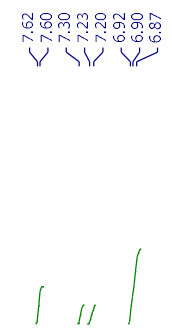

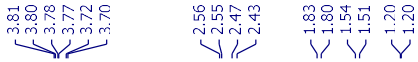

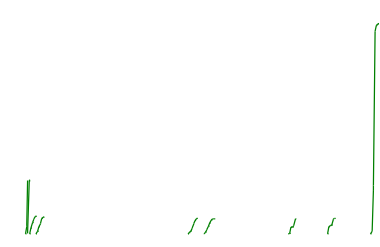

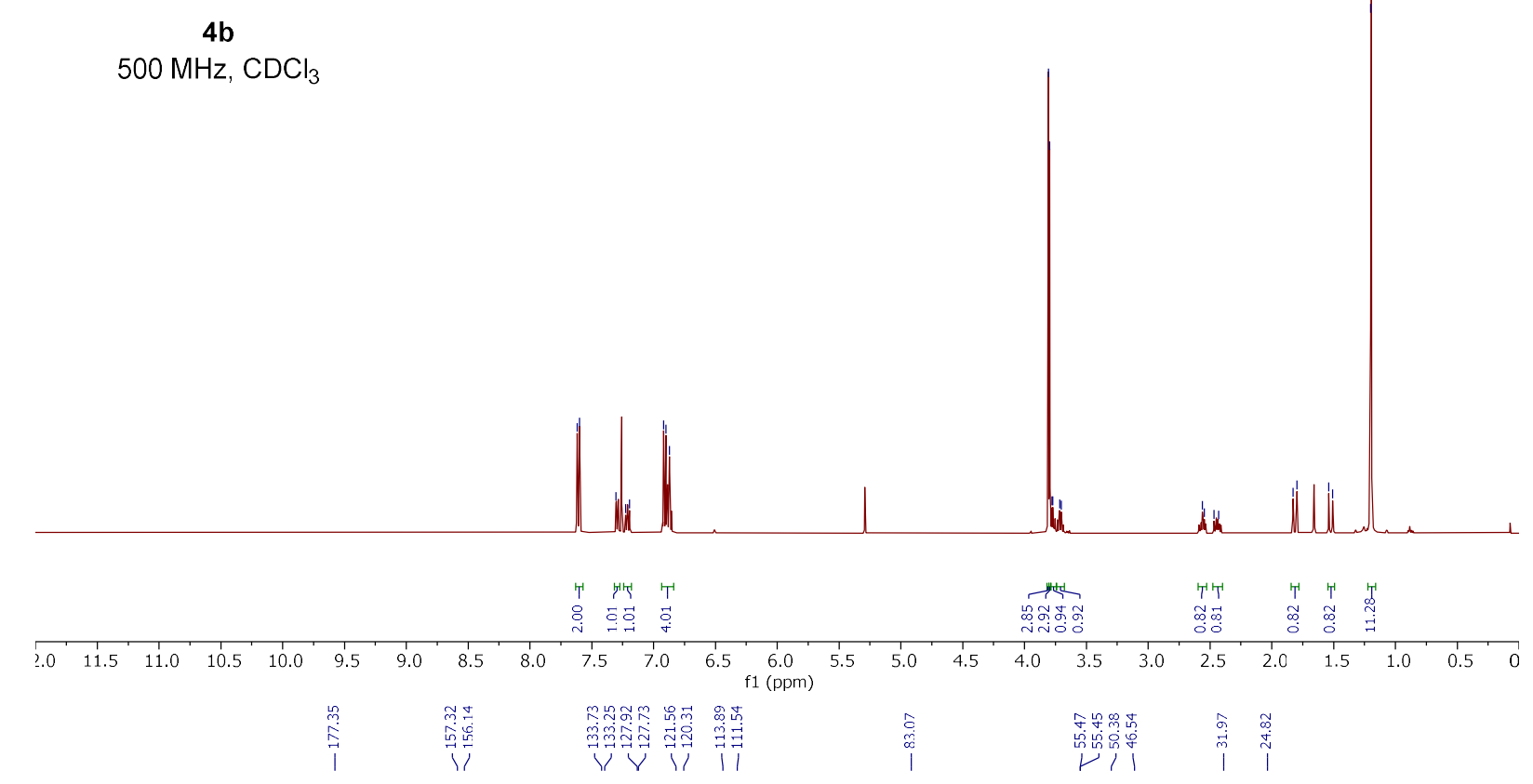

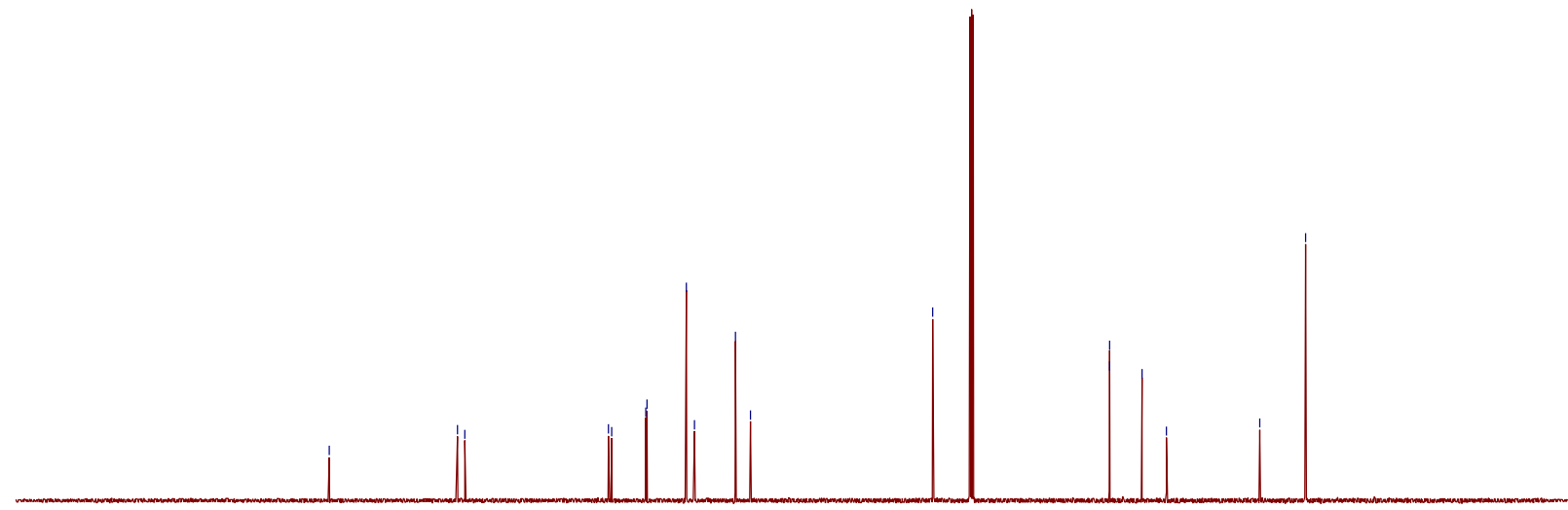

$\begin{array}{llllllllllllllllllllllll}1 & 1 \\ 220 & 210 & 200 & 190 & 180 & 170 & 160 & 150 & 140 & 130 & 120 & 110 & 100 & 90 & 80 & 70 & 60 & 50 & 40 & 30 & 20 & 10 & 0^{1} & -10\end{array}$ 


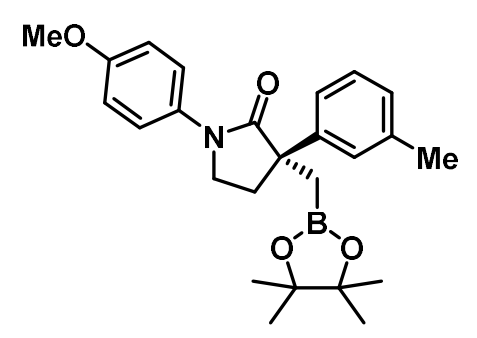

4c

$500 \mathrm{MHz}, \mathrm{CDCl}_{3}$

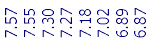 \\ परीया}

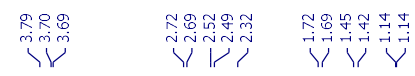

I I

$\iint$
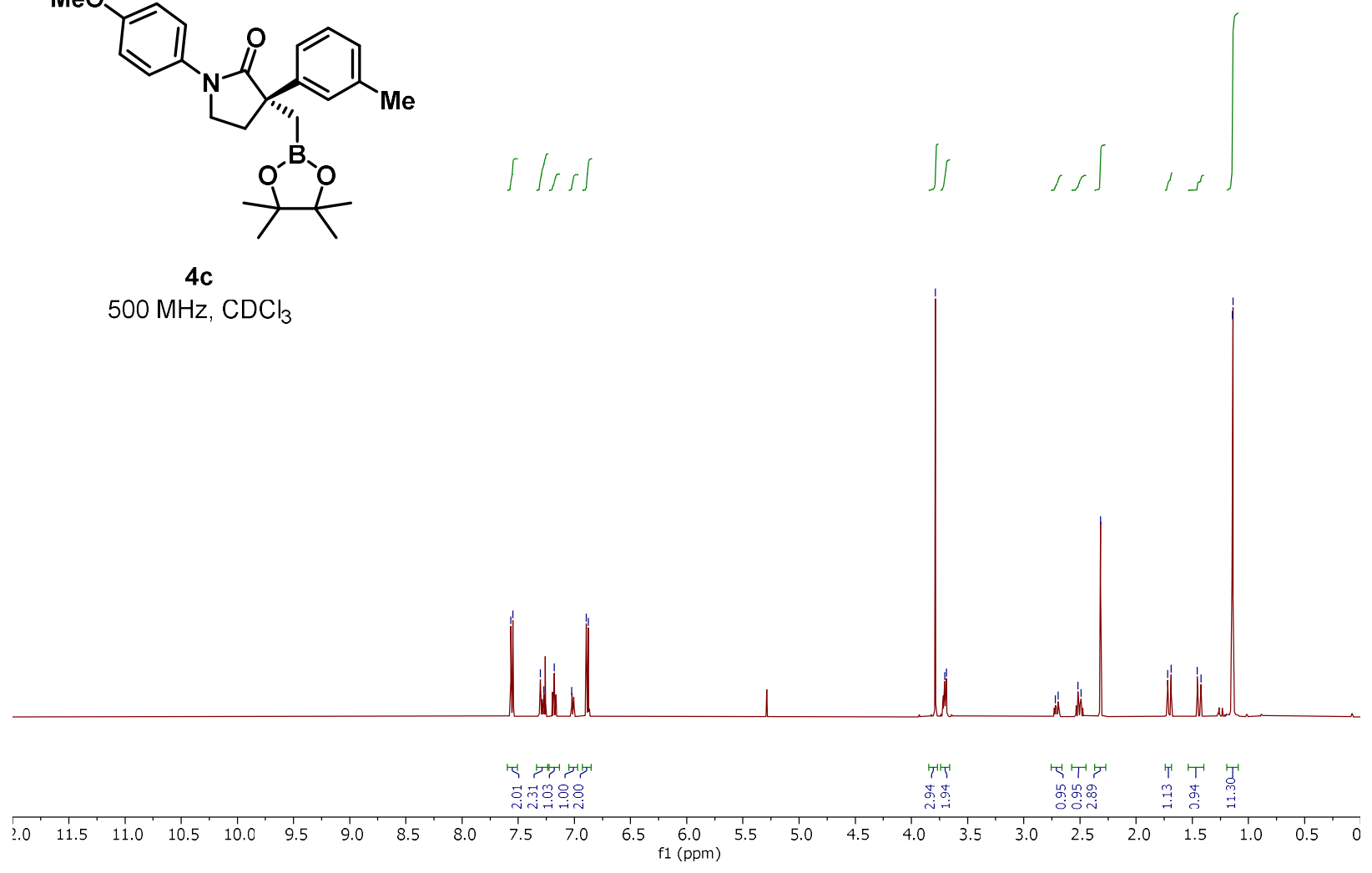

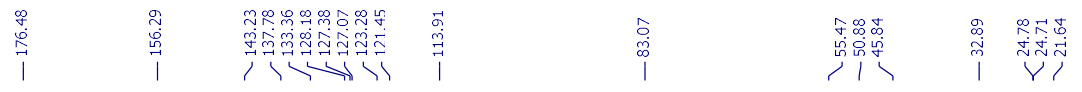

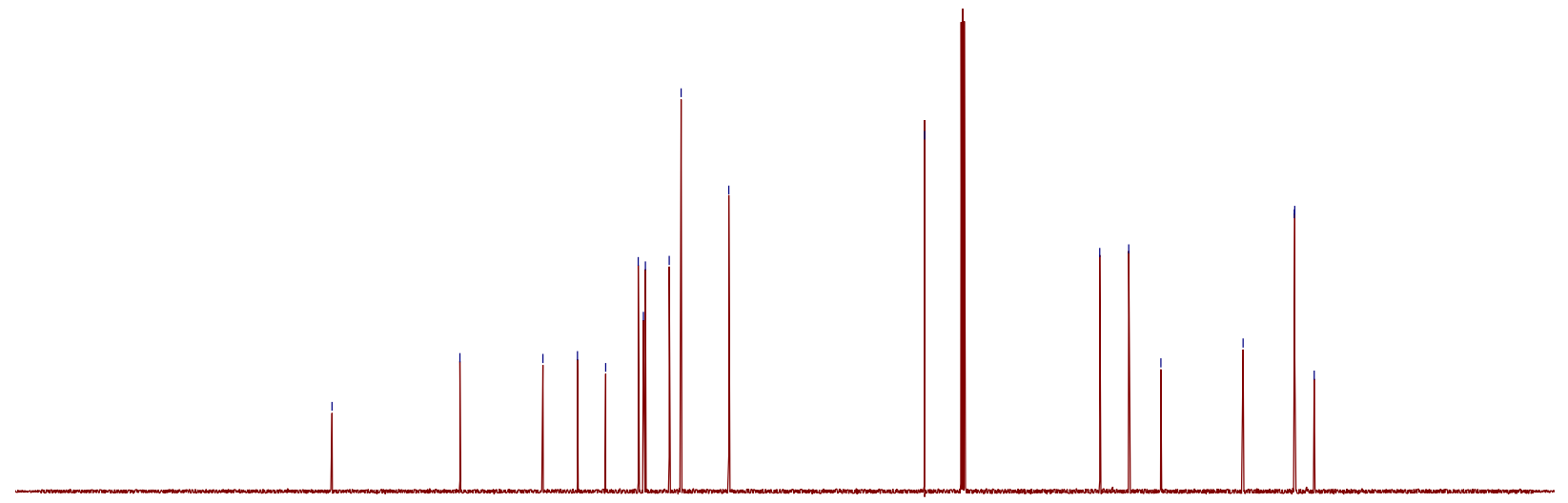

$\begin{array}{lllllllllllllllllllllllll}1 & 1 \\ 220 & 200 & 190 & 180 & 170 & 160 & 150 & 140 & 130 & 120 & 110 & 100 & 90 & 80 & 70 & 60 & 50 & 40 & 30 & 20 & 10 & 0 & -10\end{array}$ 

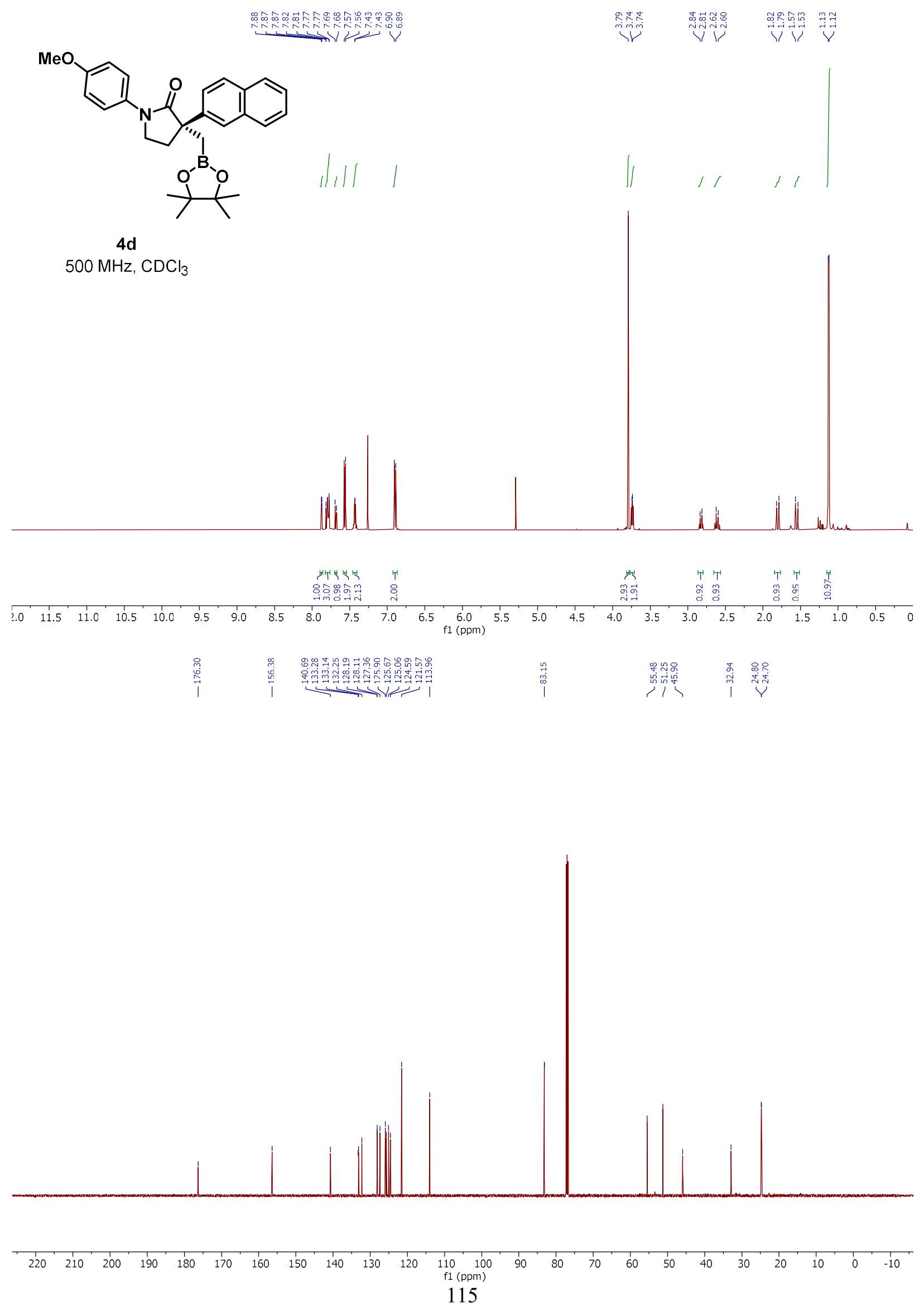


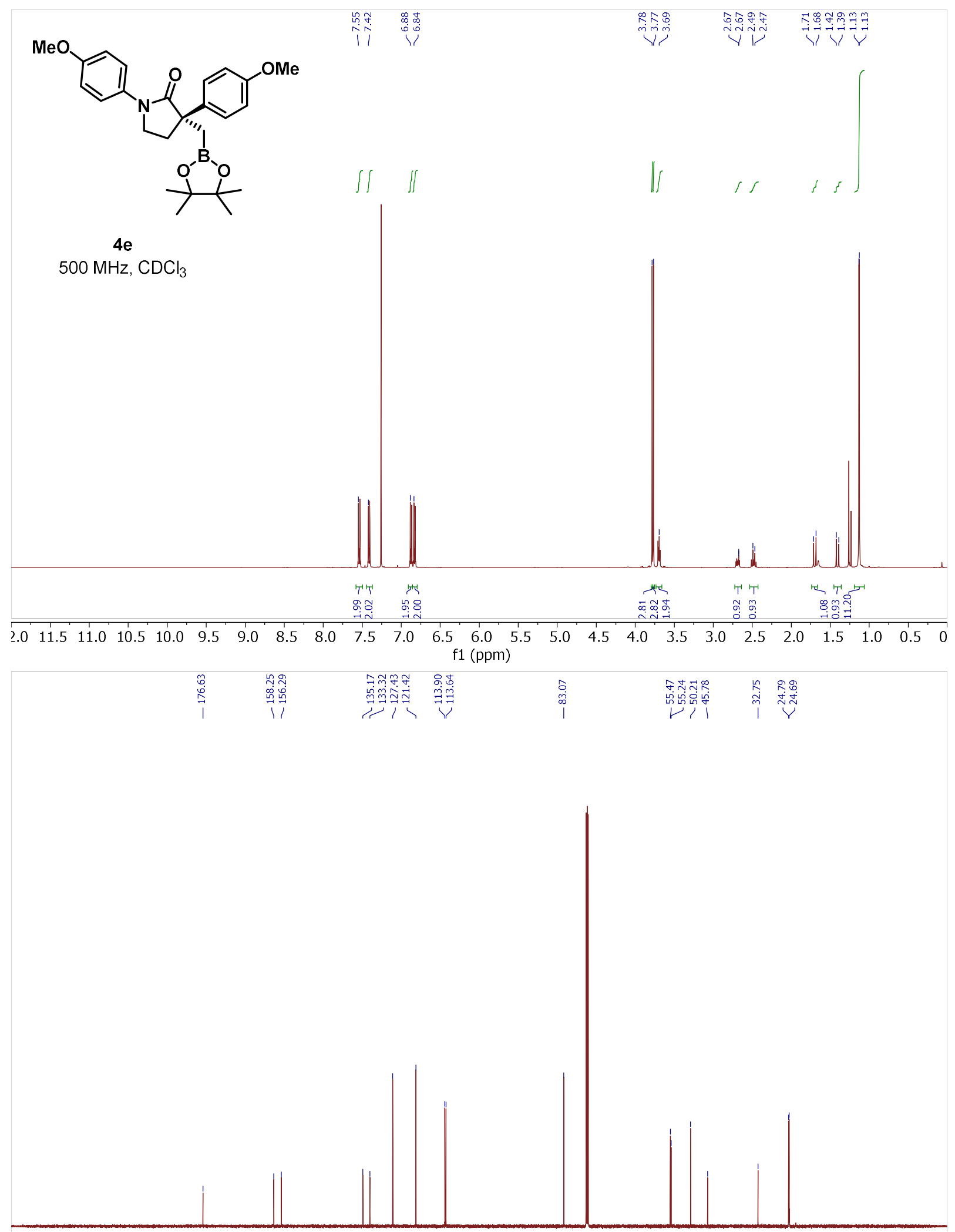

$\begin{array}{lllllllllllllllllllllllllllllll}220 & 210 & 200 & 190 & 180 & 170 & 160 & 150 & 140 & 130 & 120 & 110 & 100 & 90 & 80 & 70 & 60 & 50 & 40 & 30 & 20 & 10 & 0 & -10\end{array}$ 

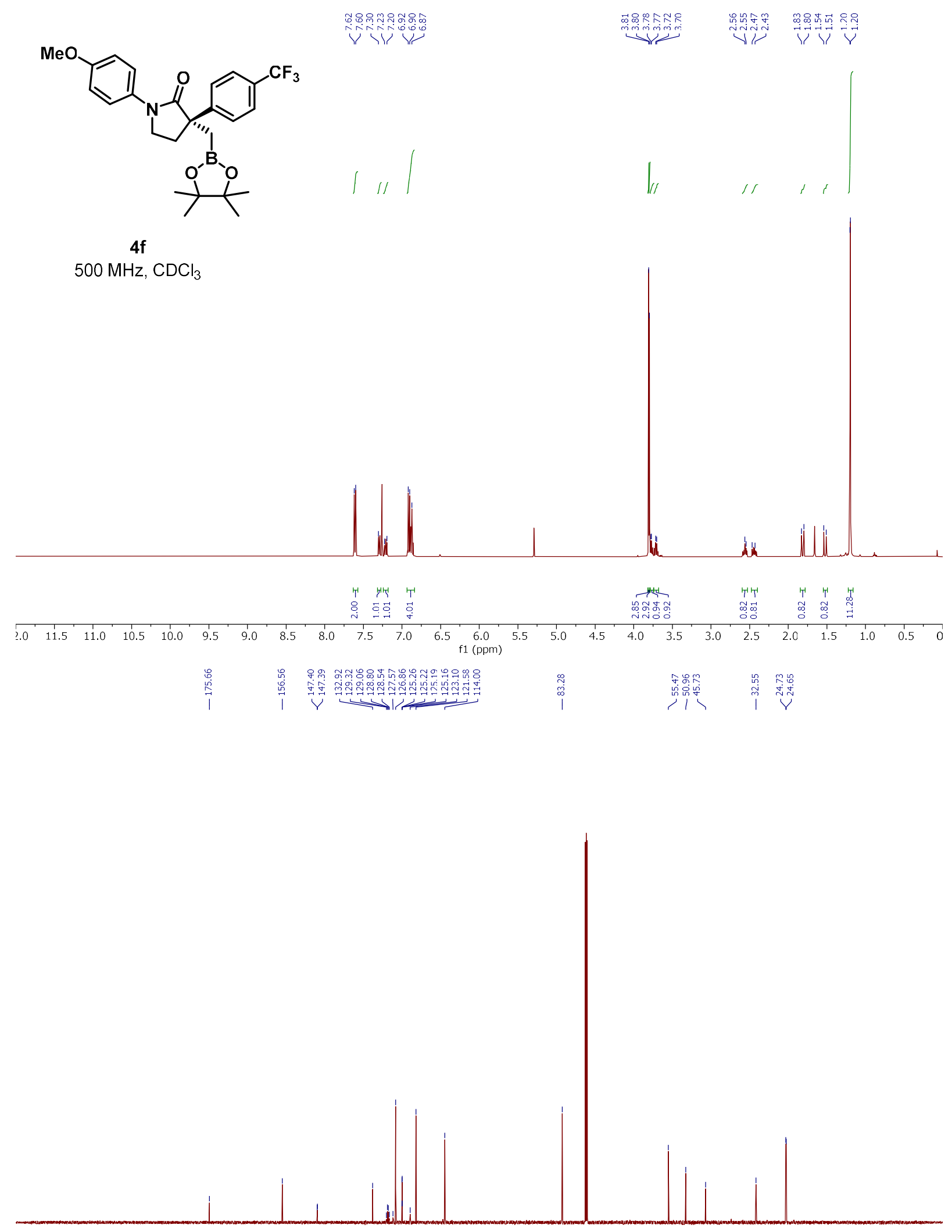

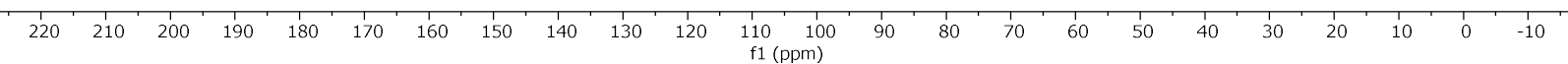


$400 \mathrm{MHz}, \mathrm{CDCl}_{3}$

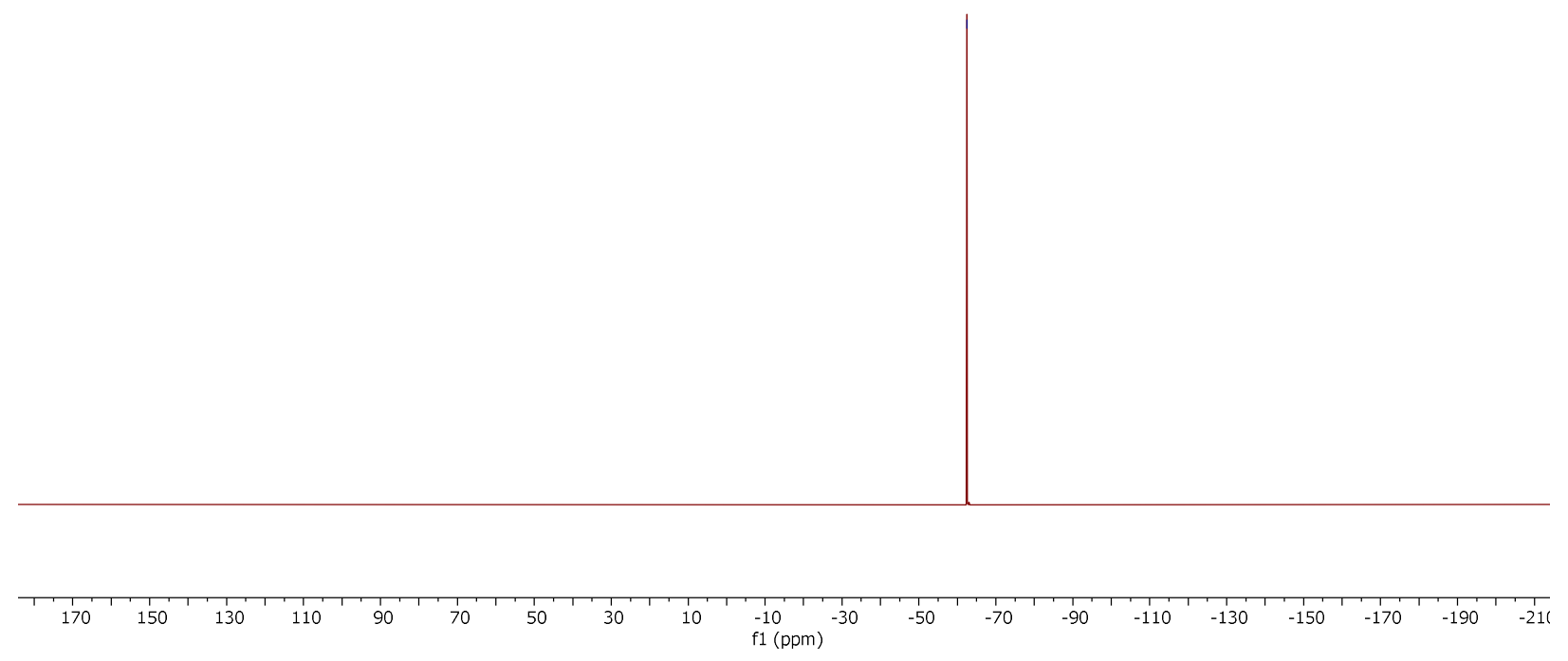



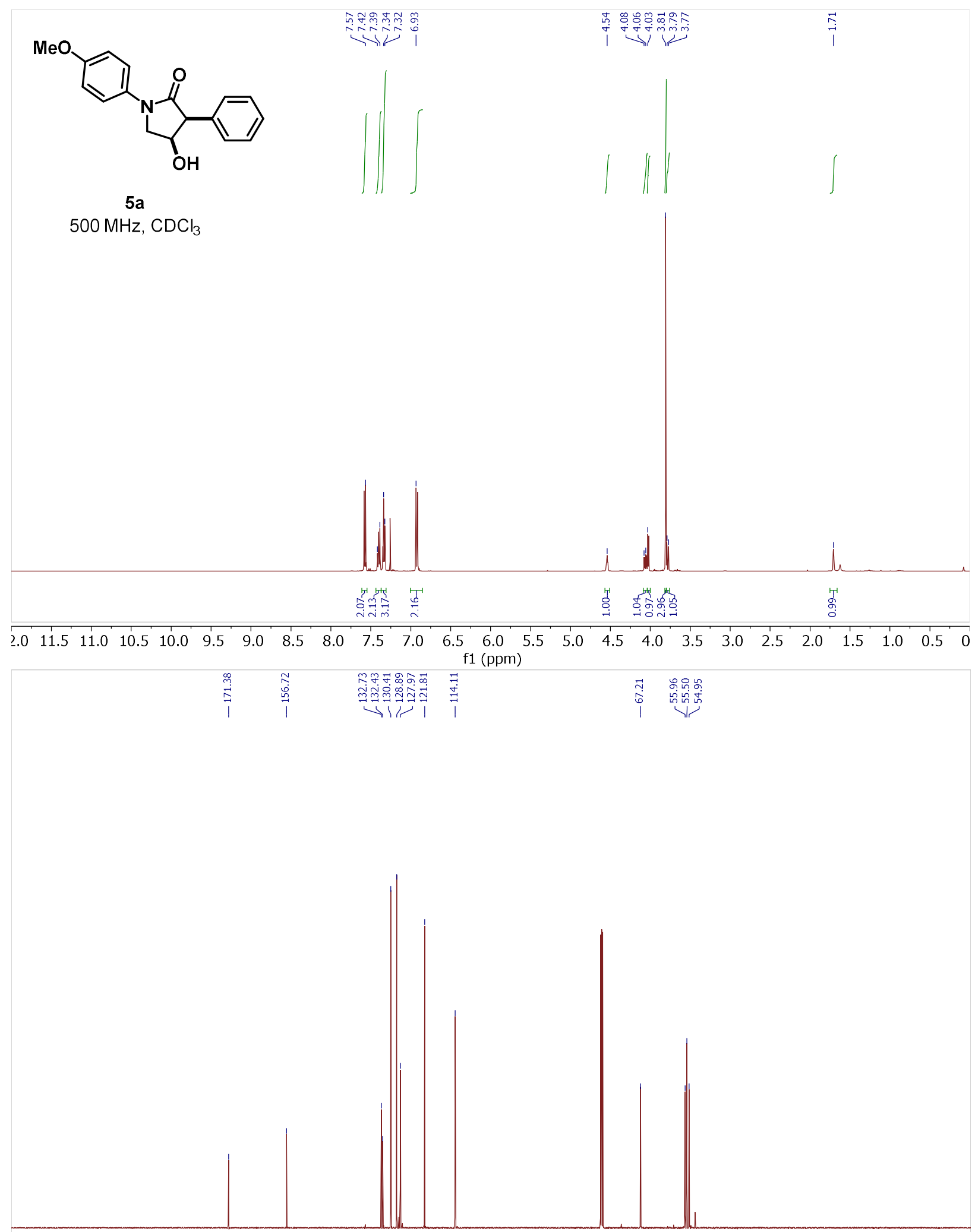

$\begin{array}{llllllllllllllllllllllll}\prime 220 & 210 & 200 & 190 & 180 & 170 & 160 & 150 & 140 & 130 & 120 & \begin{array}{l}110 \\ \mathrm{f} 1(\mathrm{ppm})\end{array} & 100 & 90 & 80 & 70 & 60 & 50 & 40 & 30 & 20 & 10 & 0 & -10\end{array}$ 


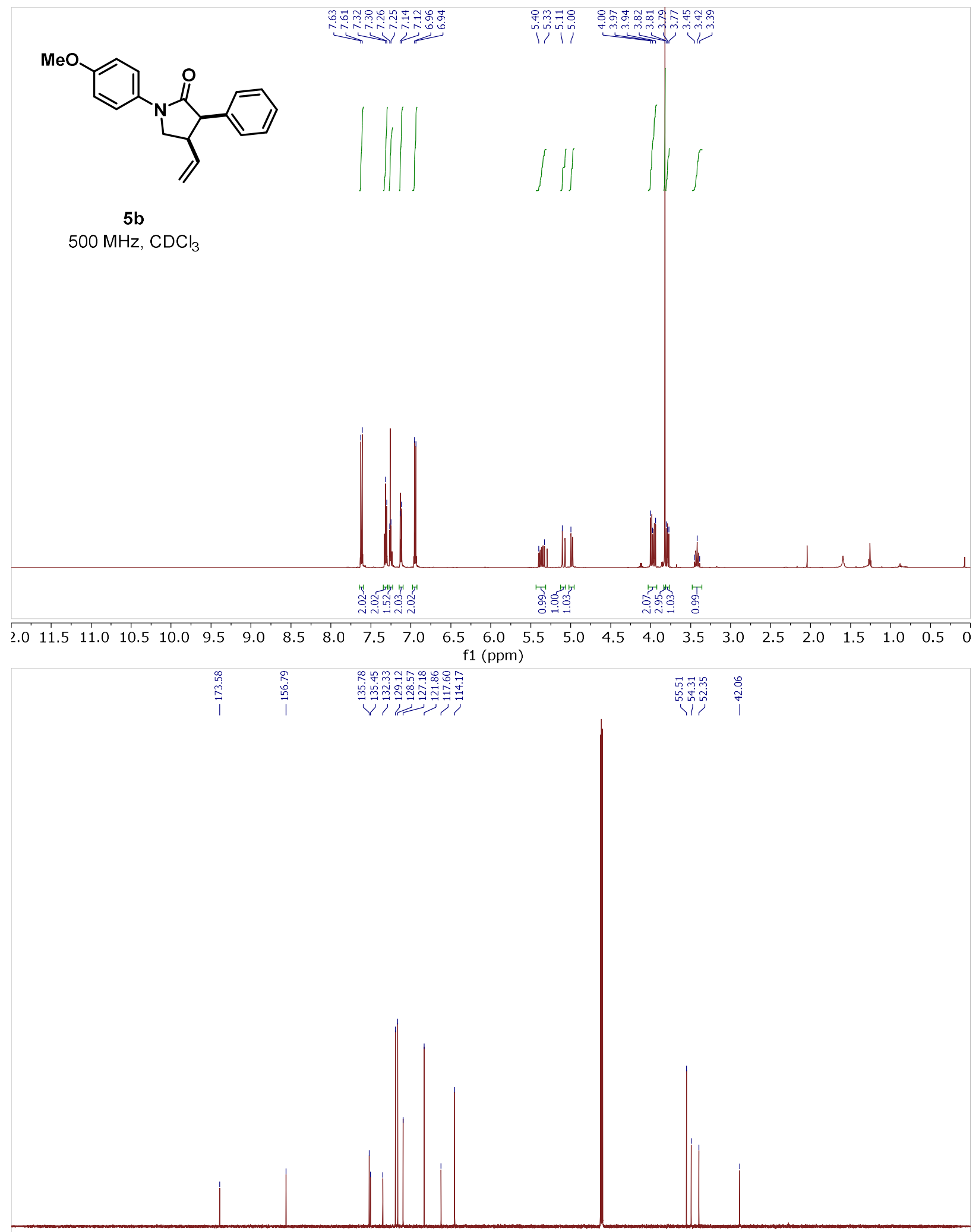

$\begin{array}{lllllllllllllllllllllllllll}220 & 210 & 200 & 190 & 180 & 170 & 160 & 150 & 140 & 130 & 120 & 110 & 100 & 90 & 80 & 70 & 60 & 50 & 40 & 30 & 20 & 10 & 0 & -10\end{array}$ 

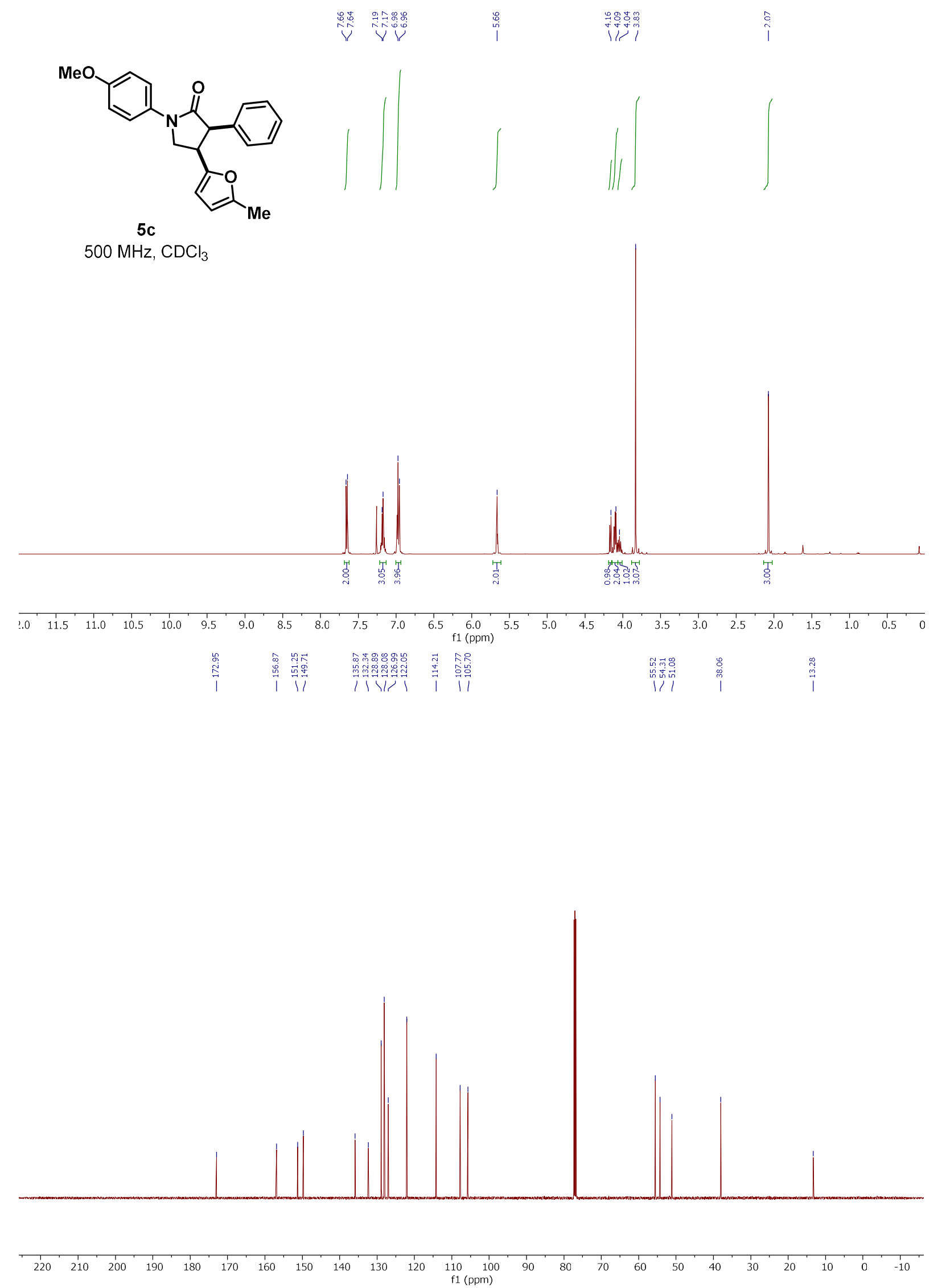
